\title{
GE-Hitachi As-Run Neutronics ECAR-4740
}

\author{
Jill R Mitchell
}

December 2019

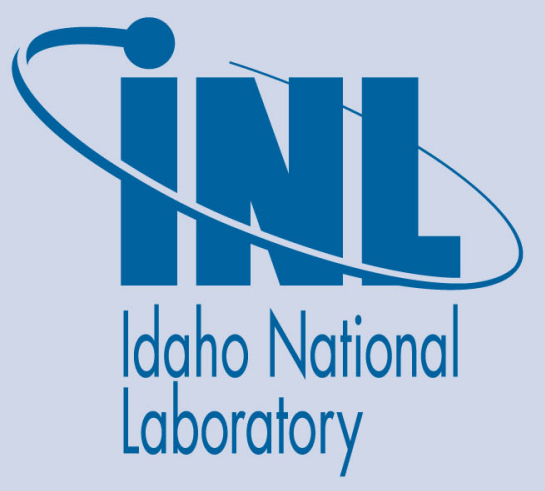

The INL is a U.S. Department of Energy National Laboratory operated by Battelle Energy Alliance 


\section{GE-Hitachi As-Run Neutronics ECAR-4740}

Jill R Mitchell

December 2019

Idaho National Laboratory Idaho Falls, Idaho 83415

http://www.inl.gov

Prepared for the

U.S. Department of Energy

Under DOE Idaho Operations Office

Contract DE-AC07-05ID14517 
Title: AS-RUN NEUTRONICS EVALUATION FOR THE GE HITACHI EXPERIMENT IN THE ATR

ECAR NO

4740
REV. NO.: 0

PROJECT NO.: 32535 Date: 12/02/2019

\begin{tabular}{|c|c|c|c|}
\hline 1. & $\begin{array}{l}\text { Does this ECAR involve a } \\
\text { Safety SSC? }\end{array}$ & No & \multirow{6}{*}{$\begin{array}{l}\text { Professional Engineer's Stamp } \\
\text { N/A } \\
\text { See LWP-10010 for requirements }\end{array}$} \\
\hline 2. & $\begin{array}{l}\text { Safety SSC Determination } \\
\text { Document ID }\end{array}$ & N/A & \\
\hline 3. & Engineering Job (EJ) No. & NA & \\
\hline 4. & SSC ID & N/A & \\
\hline 5. & Building & TRA-670 & \\
\hline 6. & Site Area & ATR Complex & \\
\hline 7. & \multicolumn{3}{|c|}{$\begin{array}{l}\text { Objective/Purpose: } \\
\text { This Engineering Calculations and Analysis Report (ECAR) documents the results of the Advanced } \\
\text { Test Reactor (ATR) detailed Monte Carlo N-Particle (MCNP) code full-core model as-run physics } \\
\text { analysis performed to support shipping and PIE for the GE-Hitachi static drop-in experiment in } \\
\text { the B-11 position (see Appendix A). }\end{array}$} \\
\hline & \multicolumn{3}{|c|}{ The purpose of this analysis is to calculate the following: } \\
\hline & \multicolumn{3}{|c|}{$\begin{array}{l}\text { - The heat-generation rates of the test specimens and associated components. } \\
\text { - The DPA for each capsule as a result of irradiation for the currently planned cycles. } \\
\text { - Decay heat (watts/cc) at shutdown and after } 30 \text { minutes, } 5 \text { hours, } 1 \text { day, } 7 \text { days, } 14 \text { days, } \\
30 \text { days, } 60 \text { days, } 90 \text { days, } 120 \text { days, and } 360 \text { days of cooling for each material. } \\
\text { - Source terms (curies) at shutdown and after } 30 \text { minutes, } 5 \text { hours, } 1 \text { day, } 7 \text { days, } 14 \text { days, } \\
30 \text { days, } 60 \text { days, } 90 \text { days, } 120 \text { days, and } 360 \text { days of cooling for the experiment. }\end{array}$} \\
\hline & $\begin{array}{l}\text { These calculations were } p \\
\text { neutronics model descrip }\end{array}$ & $\begin{array}{l}\text { med using th } \\
\text { analysis detai }\end{array}$ & $\begin{array}{l}\text { codes MCNP and ORIGEN2. The } \\
\text { Its are presented in the attached report. }\end{array}$ \\
\hline
\end{tabular}


TEM-10200-1

$12 / 19 / 17$

ENGINEERING CALCULATIONS AND ANALYSIS

Rev.08

Title: $\quad$ AS-RUN NEUTRONICS EVALUATION FOR THE GE HITACHI EXPERIMENT IN THE ATR

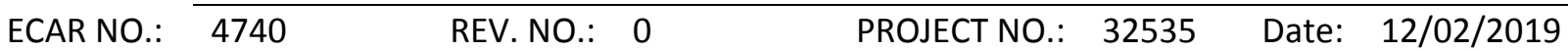

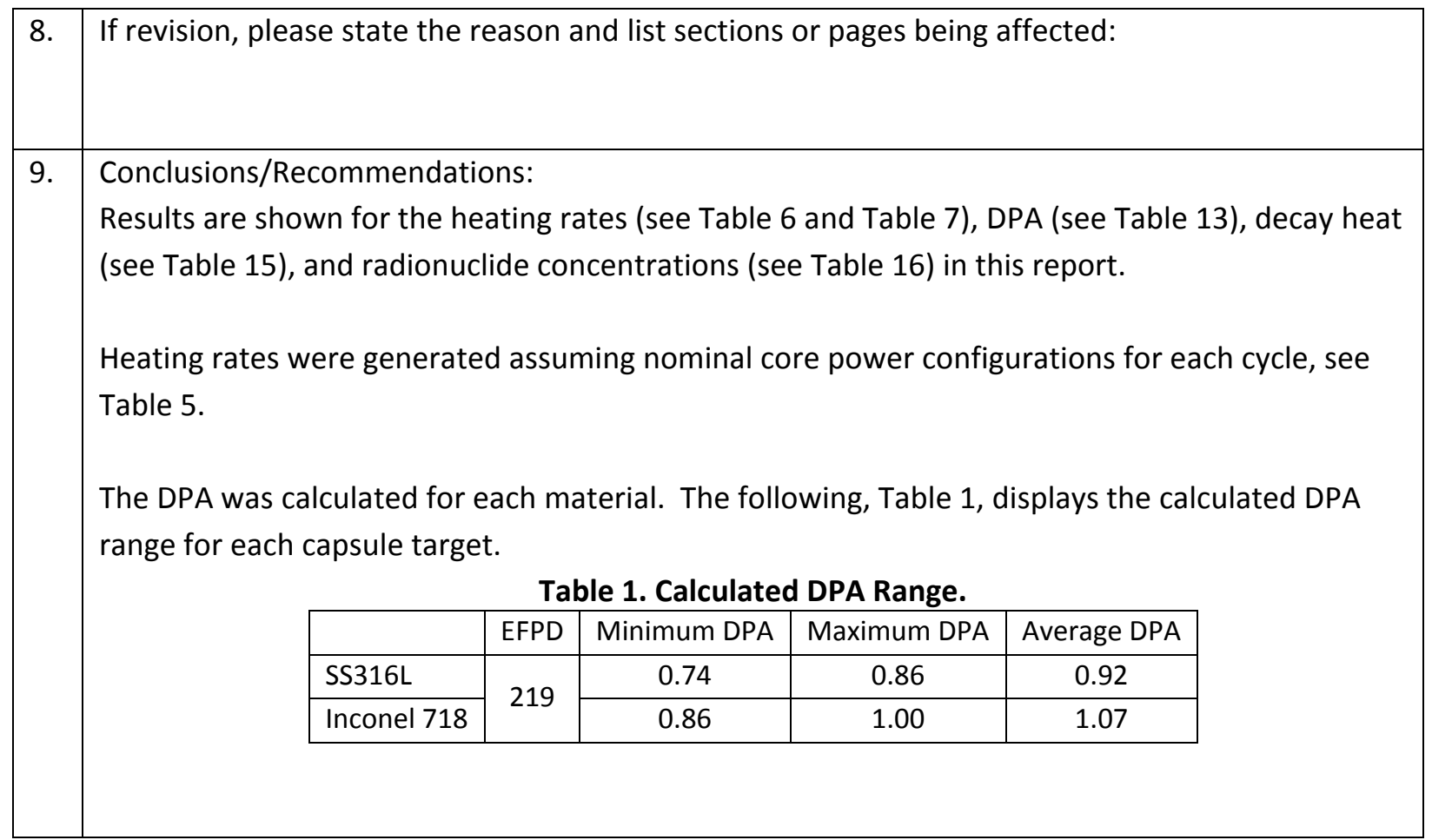


TEM-10200-1

$12 / 19 / 17$

ENGINEERING CALCULATIONS AND ANALYSIS

Page 3 of 57

Rev.08

Title: $\quad$ AS-RUN NEUTRONICS EVALUATION FOR THE GE HITACHI EXPERIMENT IN THE ATR

\begin{tabular}{|c|c|c|c|c|c|}
\hline ECAR NO.: & 4740 & REV. NO.: & PROJECT NO.: & 32535 & Date: $12 / 02 / 2019$ \\
\hline
\end{tabular}

\section{Contents}

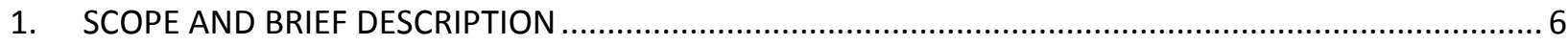

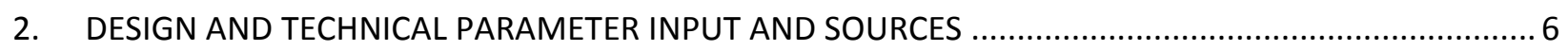

3. RESULTS OF LITERATURE SEARCHES AND OTHER BACKGROUND DATA …................................... 9

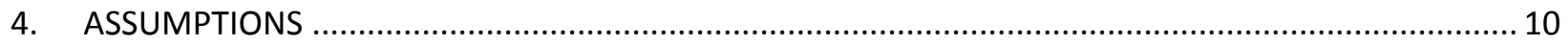

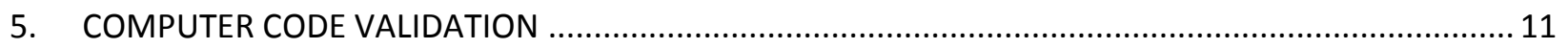

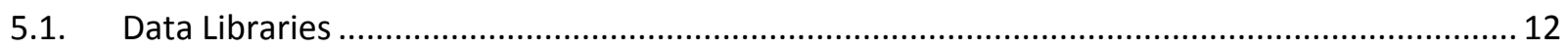

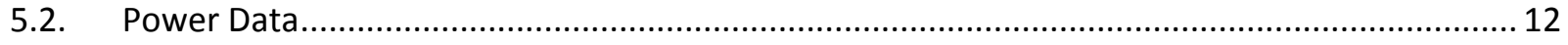

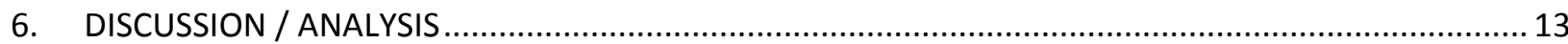

6.1. Neutron/Prompt Gamma Heating and Delayed Fission Product Gamma Heating Normalization Factors 13

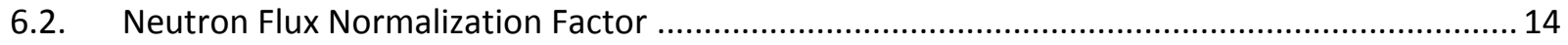

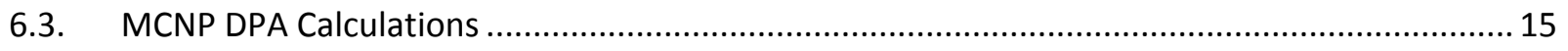

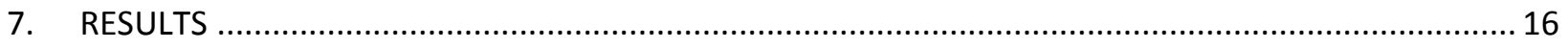

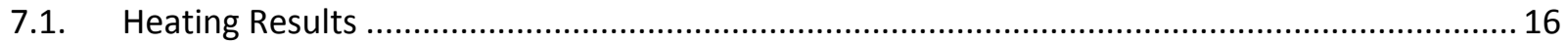

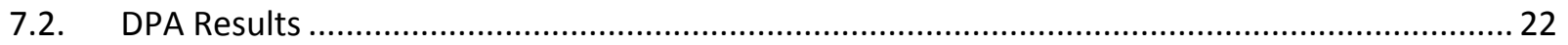

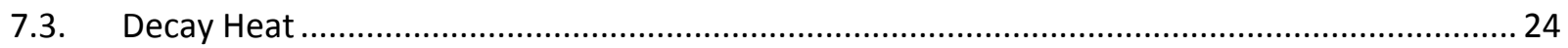

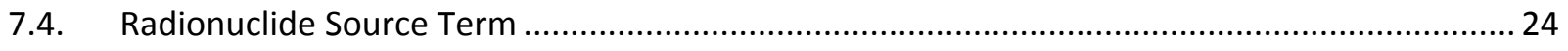

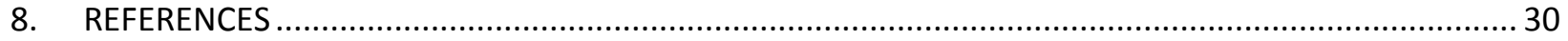

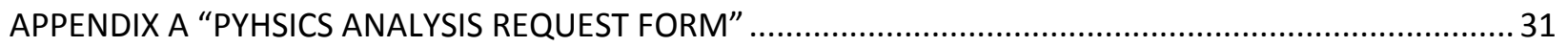

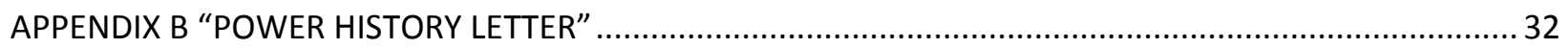

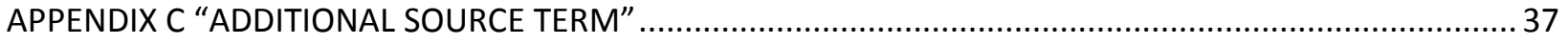


TEM-10200-1

$12 / 19 / 17$

Rev.08

Title: $\quad$ AS-RUN NEUTRONICS EVALUATION FOR THE GE HITACHI EXPERIMENT IN THE ATR

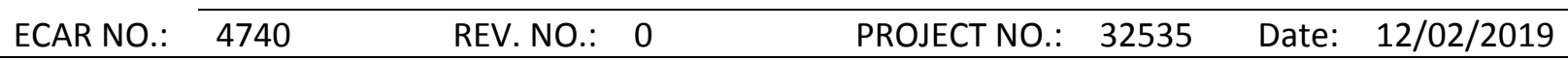

\section{List of Tables}

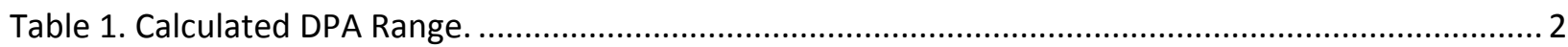

Table 2. List of drawings for GE-HITACHI experiment. .................................................................... 9

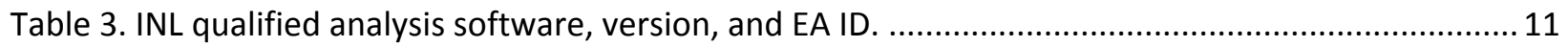

Table 4. Computer configurations for INL-qualified MCNP5 and ORIGEN2 installations......................... 11

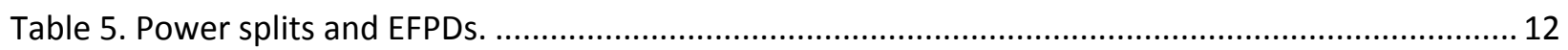

Table 6. Pressure Boundary Internal Component, End Cap, and Spacer Heat Generation Rates. ............. 17

Table 7. Beryllium Surrounding B-11 Position Heat Generation Rates................................................... 18

Table 7. Water Annulus Outside Basket Heat Generation Rates.......................................................... 19

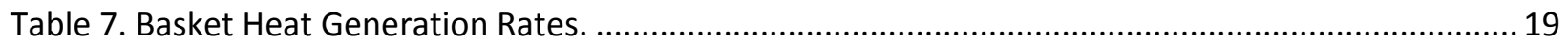

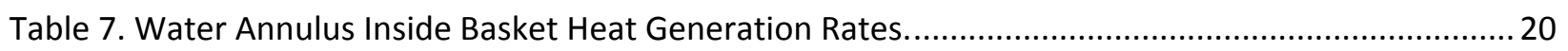

Table 7. Pressure Boundary Heat Generation rates. .......................................................................... 21

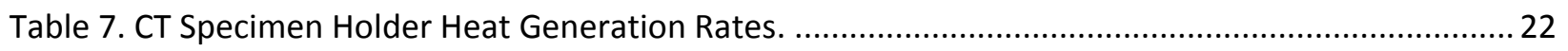

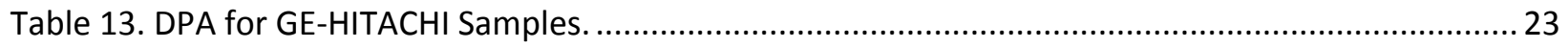

Table 13. DPA for all SS316L and Inconel 718 Specimens after four cycles of irradiation. ........................23

Table 9. Volumetric Decay Heat for Experiment Materials..................................................................2 24

Table 11. Radionuclide source term for the GE-HITACHI experiment after 219 days of irradiation (Ci). .. 25 
Rev.08

Title: $\quad$ AS-RUN NEUTRONICS EVALUATION FOR THE GE HITACHI EXPERIMENT IN THE ATR

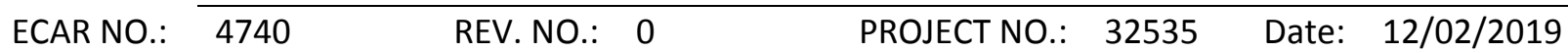

\section{PROJECT ROLES AND RESPONSIBILITIES}

\begin{tabular}{l|l|l|l} 
Project Role & Name (Typed) & Organization & Pages Covered (if applicable) \\
\hline $\begin{array}{l}\text { Performer(s) } \\
\text { Checker }\end{array}$ & Jill Mitchell & C-130 & All \\
Independent & Brian Gross & C-130 & All \\
$\begin{array}{l}\text { Reviewer } \\
\text { CUl Reviewer }\end{array}$ & N/A & -- & -- \\
Manager $^{\text {d }}$ & Matthew Arrowood & C-601 & All \\
Requestor & Misti Lillo & C-130 & All \\
Nuclear Safety & Matthew Arrowood & C-601 & All \\
Document Owner & N/A & -- & -- \\
& John Jackson & C-002 & All
\end{tabular}

\section{Responsibilities:}

\footnotetext{
a. Confirmation of completeness, mathematical accuracy, and correctness of data and appropriateness of assumptions.

b. Concurrence of method or approach. See definition, LWP-10106.

c. Concurrence with the document's markings in accordance with LWP-11202.

d. Concurrence of procedure compliance. Concurrence with method/approach and conclusion.

e. Concurrence with the document's assumptions and input information. See definition of Acceptance, LWP-10200.
}

NOTE: $\quad$ Delete or mark "N/A" for project roles not engaged. Include ALL personnel and their roles listed above in the eCR system. The list of the roles above is not allinclusive. If needed, the list can be extended or reduced. 
Rev.08

Title: $\quad$ AS-RUN NEUTRONICS EVALUATION FOR THE GE HITACHI EXPERIMENT IN THE ATR

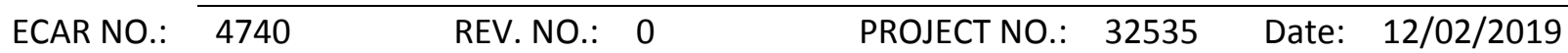

\section{SCOPE AND BRIEF DESCRIPTION}

This engineering calculations and analysis report (ECAR) documents the results of the Advanced Test Reactor (ATR) as-run physics analysis performed to support shipping and PIE of the GE Hitachi static drop-in experiment in the B-11 position located in the south region of the ATR. The purpose of this analysis is to calculate the following:

- The as-run neutron and gamma heat rates for the GE Hitachi experiment;

- The DPA for each capsule as a result of irradiation;

- Decay heat (watts) at shutdown and after 30 minutes, 5 hours, 1 day, 7 days, 14 days, 30 days, 60 days, and 360 days of cooling for each material;

- Source term (curies) at shutdown and after 30 minutes, 5 hours, 1 day, 7 days, 14 days, 30 days, 60 days, and 360 days of cooling for the experiment.

\section{DESIGN AND TECHNICAL PARAMETER INPUT AND SOURCES}

The GE Hitachi experiment was irradiated in the B-11 position in the ATR, shown in Figure 1 . The experiment specimens consisted of twelve rounded, compact tension (CT) specimens, six composed of SS316L and six of Inconel 718, and eight ASTM standard sub-size tensile specimens, four composed of SS316L and four Inconel 718, packaged inside three specimen holders centered about the ATR core midplane and were "clocked" to ensure the tension specimens were oriented facing the ATR core center and the tensile specimens. To ensure proper tensile specimen vertical alignment within each holder, a sleeve surrounds the smaller diameter portion of each tensile specimen. ECAR-3569 [[14]] contains a detailed description of the GE-Hitachi experiment model. Figure 2 shows an axial view of the MCNP model of the experiment assembly. 
TEM-10200-1

$12 / 19 / 17$

Rev.08

Title: $\quad$ AS-RUN NEUTRONICS EVALUATION FOR THE GE HITACHI EXPERIMENT IN THE ATR

$\begin{array}{llllllll}\text { ECAR NO.: } & 4740 & \text { REV. NO.: } & 0 & \text { PROJECT NO.: } & 32535 & \text { Date: } & \text { 12/02/2019 }\end{array}$

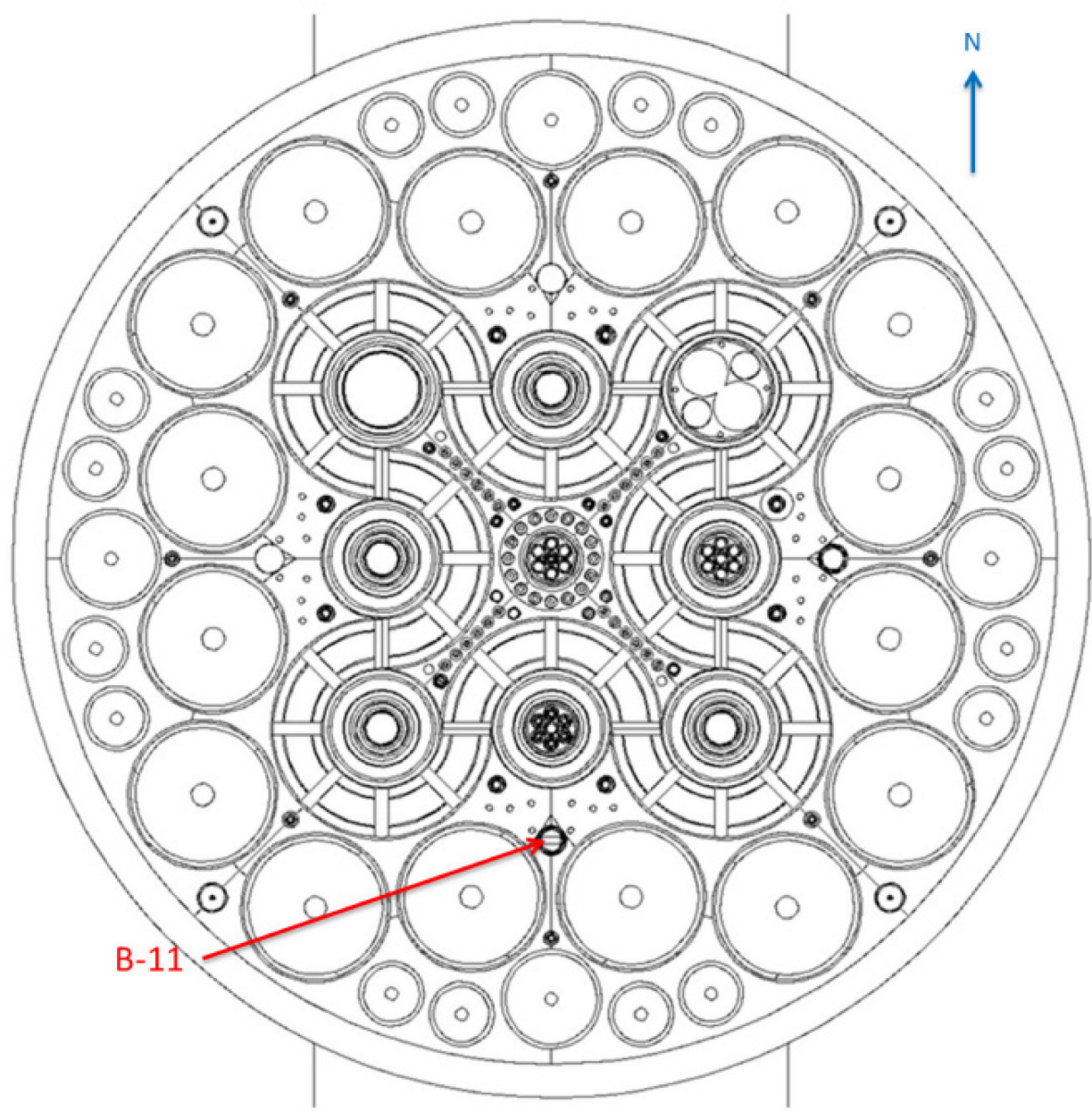

Figure 1. Radial cross section view of the ATR core, B-11 irradiation test position. 
TEM-10200-1

$12 / 19 / 17$

Rev.08

Title: $\quad$ AS-RUN NEUTRONICS EVALUATION FOR THE GE HITACHI EXPERIMENT IN THE ATR ECAR NO.: 4740 REV. NO.: 0 PROJECT NO.: 32535 Date: $12 / 02 / 2019$

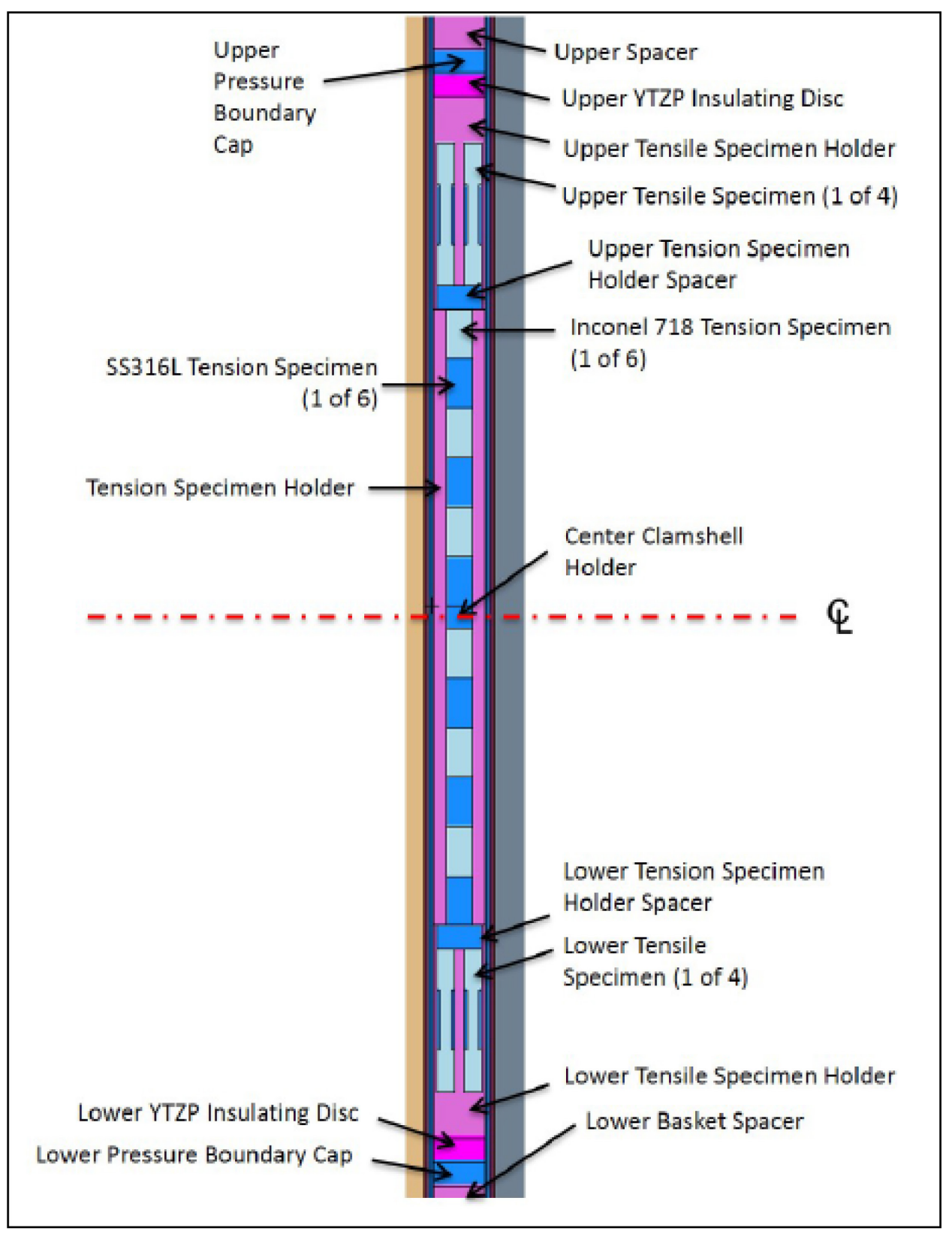

Figure 2. Axial View of MCNP Model of GE Hitachi Experiment Assembly. 
Rev.08

Title: $\quad$ AS-RUN NEUTRONICS EVALUATION FOR THE GE HITACHI EXPERIMENT IN THE ATR

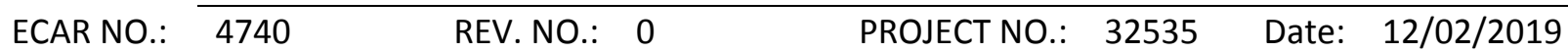

\section{RESULTS OF LITERATURE SEARCHES AND OTHER BACKGROUND DATA}

The general purpose Monte Carlo $\underline{N}$-Particle transport code, MCNP [4][5], was used to model and evaluate the GE-HITACHI experiment. ORIGEN2 [6] was used to calculate the source term for the experiment.

The model for the GE-HITACHI experiment is based on the drawings listed in Table 2. Nominal dimensions for the specimen, holders, and capsules are used in the model.

Table 2. List of drawings for GE-HITACHI experiment.

\begin{tabular}{|c|c|l|}
\hline $\begin{array}{c}\text { INL } \\
\text { Drawing }\end{array}$ & Rev. & \\
\hline 605754 & 1 & ATR GE HITACHI (GEH) CAPSULE DETAILS AND ASSEMBLY \\
\hline 605755 & 1 & ATR GE HITACHI (GEH) ADDITIVELY MANUFACTURED (AM) ROUND TENSILE SPECIMEN DETAIL \\
\hline 605756 & 1 & $\begin{array}{l}\text { ATR GE HITACHI (GEH) ADDITIVEVLY MANUFACTURED (AM) COMPACT TENSILE SPECIMEN } \\
\text { DETAIL }\end{array}$ \\
\hline 605757 & 2 & ATR GE HITCAHI (GEH) FINAL ASSMBLY \\
\hline 605758 & 1 & ATR GE HITACHI (GEH) BASKET DETAILS AND ASSEMBLY \\
\hline 605759 & 2 & ATR GE HITACHI (GEH) PRESSURE BOUNDARY AND SPACERS DETAILS \\
\hline 605760 & 0 & ATR GE HITACHI (GEH) IN-VESSEL INSTALLATION \\
\hline
\end{tabular}

The files used for this analysis are located on the Irradiation Testing server [Z:YIRRADIATION TESTING \C660 Analysts\Jill Mitchell files\Projects\2019\GE-HIT AsRun\cksum_final_files\New_final]. The following checksum results are provided for the supporting documents:

38194342611802 316SS_long.inp

9083776151789944 316SS_long.out

24169651422002 Al_6061.inp

243878177585472 Al_6061.out

41142817131291360 Cycle162A.xlsx

$24701641831421 \mathrm{Fe}$ _long.inp

20525881751691250 Fe_long.out

3450685755453236 GE_AsRun_JRM1.xlsx

31171612571192473 ged

12374720011192202 ged1
20819782397778763 ged1.o

15032901567778761 ged.o

21865520411198601 gedpa

117866245914005626 gedpa.o

9829570081209226 geh1

31879858691209497 geh1 (2)

164807929714207487 geh1 (2).o

12318185914207487 geh1.o

27778238011198872 grdpa

198900503714005626 grdpa.o 
Rev.08

Title: $\quad$ AS-RUN NEUTRONICS EVALUATION FOR THE GE HITACHI EXPERIMENT IN THE ATR

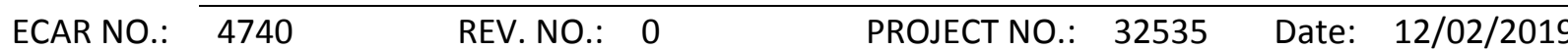

13649226502215 Inconel_718.inp

1736015861101340 Inconel_718.out

16168168142058 incon_long.inp

9519336701830072 incon_long.out

$18403089861421 \mathrm{Nb}$ _long.inp

$38959380051690690 \mathrm{Nb}$ _long.out

5770401192031 SS316L.inp

185654342293075 SS316L.out
10907611481421 Ti_long.inp

26290220301697148 Ti_long.out

14585663543749

Total_experiment_assembly.inp

572013508118681

Total_experiment_assembly.out

27234650911849 YTZP_zirconia.inp

2400910794300126 YTZP_zirconia.out

\section{ASSUMPTIONS}

The following assumptions were used in this analysis:

1. The as-run analysis was performed based on nominal lobe powers provided in Appendix $B$ for 162A through 164B.

2. An increase in lobe power for any lobe affecting the south region of the reactor will result in a change in test heating. Therefore, any power changes must be accounted for as a function of the ratio of the actual south lobe power to the analyzed south lobe source power.

3. The south lobe power is defined by the average of the SW, SE, and C lobe powers; $\mathrm{S}=(\mathrm{SW}+\mathrm{SE}+\mathrm{C}) / 3$.

4. Heating rate values reported for GE-HITACHI include energy deposition from prompt neutrons, prompt gammas, and delayed fission product gammas.

5. Contributions to heat and radionuclide source term from the flux wires, melt wires, and quartz tubes are negligible.

6. The radionuclide source term was calculated using the maximum neutron flux seen by the experiment which was scaled to the maximum south source power of $26.4 \mathrm{MW}$ assumed for cycle 162A [14]. The historical 8.5\% instrumentation uncertainty and OSCC multiplier of 1.2 for the large-B positions were added to the calculation. 
TEM-10200-1

$12 / 19 / 17$

ENGINEERING CALCULATIONS AND ANALYSIS

Page 11 of 57

Rev.08

Title: $\quad$ AS-RUN NEUTRONICS EVALUATION FOR THE GE HITACHI EXPERIMENT IN THE ATR

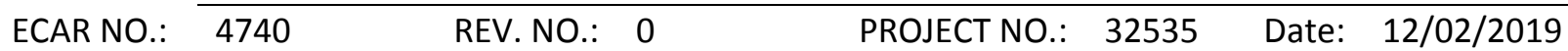

7. No credit is given to radioactive decay between cycles when calculating the radionuclide source term and decay heat.

8. YTZP ceramic material contains $3 \mathrm{wt} \% \mathrm{HfO} 2$ which represents the maximum $\mathrm{Hf}$ impurity content instead of the average of $2 \mathrm{wt} \%$ as reported by the manufacturer.

\section{COMPUTER CODE VALIDATION}

The computer code MCNP is listed in the INL Enterprise Architecture (EA) Repository and is accepted as a qualified scientific and engineering analysis software. Table 3 lists the version and EA identification (ID) for the computer code used to perform the calculations and analyses documented by this ECAR.

Table 3. INL qualified analysis software, version, and EA ID.

\begin{tabular}{|c|c|c|}
\hline Code Name & Version & V\&V Tracking Number \\
\hline MCNP & 5 (Release 1.60) & 234728 [7] \\
\hline ORIGEN2 & 2.2 & $201298[12]$ \\
\hline
\end{tabular}

MCNP Version 5, Release 1.60 and ORIGEN2 have been verified and validated (V\&V'd) for use at INL, as documented by TEV-2944 [15]. The computer configurations listed in Table 4 were used to perform the MCNP5 and ORIGEN2 calculations reported in this ECAR.

Table 4. Computer configurations for INL-qualified MCNP5 and ORIGEN2 installations.

Computer $\quad$ Processor

Operating System 
TEM-10200-1

$12 / 19 / 17$

ENGINEERING CALCULATIONS AND ANALYSIS

Page 12 of 57

Rev.08

Title: $\quad$ AS-RUN NEUTRONICS EVALUATION FOR THE GE HITACHI EXPERIMENT IN THE ATR

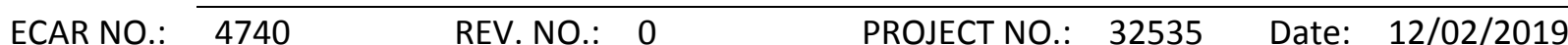

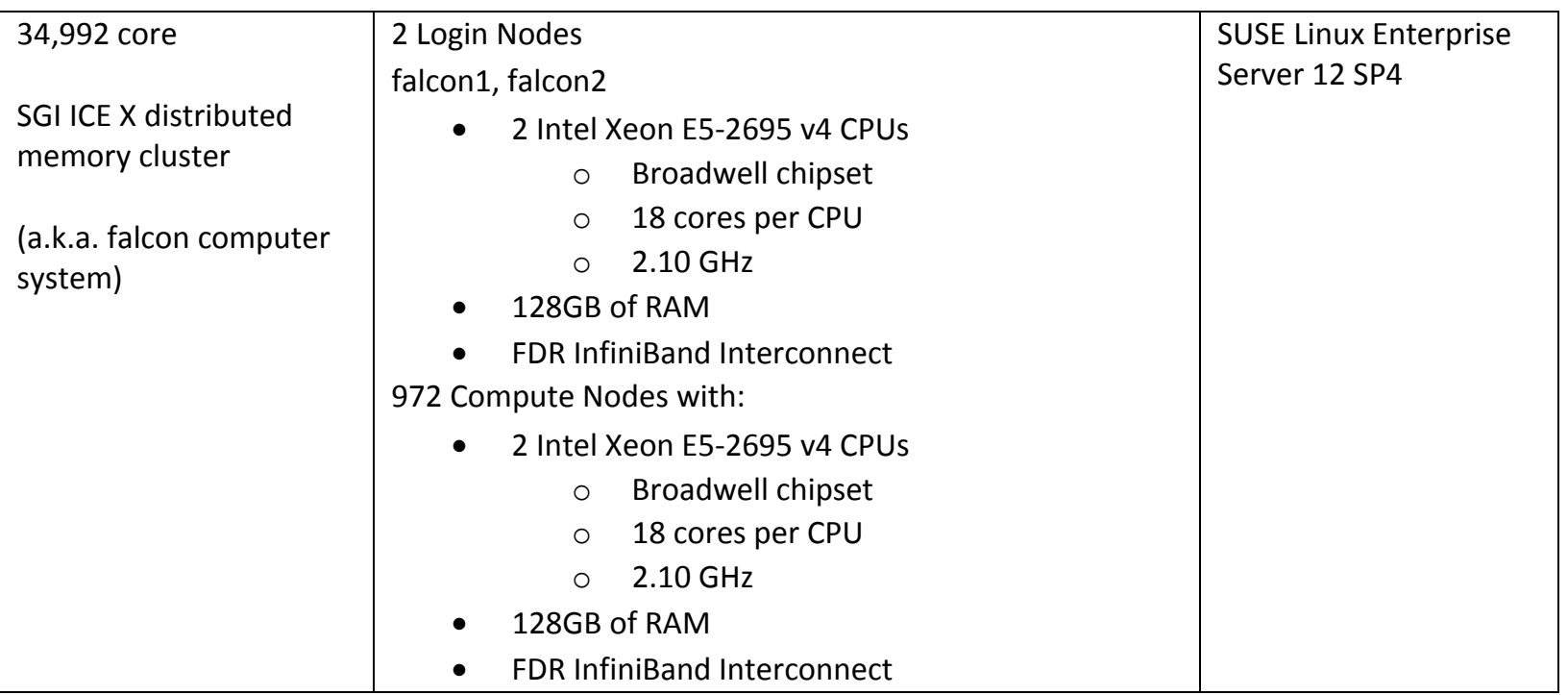

\subsection{Data Libraries}

The standard MCNP cross-section data libraries [4][5] were used to calculate the heating rates, neutron flux, and DPA for the GE-HITACHI experiment. The ENDF/B-VII library was used in the MCNP models. The ATRXS library [11] was used in the ORIGEN2 calculations.

\subsection{Power Data}

The analysis was performed using nominal lobe powers for each of the cycles. Table 5 gives a summary of the cycle data.

Table 5. Power splits and EFPDs.

\begin{tabular}{|c|c|c|c|c|c|c|c|c|}
\hline & $\begin{array}{c}\text { NW } \\
(\mathrm{MW})\end{array}$ & $\begin{array}{c}\text { NE } \\
(\mathrm{MW})\end{array}$ & $\begin{array}{c}\mathrm{C} \\
(\mathrm{MW})\end{array}$ & $\begin{array}{c}\text { SW } \\
(\mathrm{MW})\end{array}$ & $\begin{array}{c}\text { SE } \\
(\mathrm{MW})\end{array}$ & $\begin{array}{c}\text { Total } \\
(\mathrm{MW})\end{array}$ & $\begin{array}{c}\text { S } \\
(\mathrm{MW})\end{array}$ & EFPD \\
\hline $162 \mathrm{~A}$ & 20.1 & 17.9 & 22.8 & 24.0 & 26.2 & 111.0 & 24.3 & 61.9 \\
\hline $162 \mathrm{~B}$ & 20.0 & 14.5 & 19.2 & 23.9 & 23.0 & 100.6 & 22.0 & 38.5 \\
\hline $164 \mathrm{~A}$ & 20.0 & 16.0 & 19.4 & 22.4 & 25.7 & 103.5 & 22.5 & 54.9 \\
\hline $164 \mathrm{~B}$ & 19.5 & 16.4 & 19.7 & 23.1 & 25.1 & 103.8 & 22.6 & 64.1 \\
\hline \multicolumn{3}{|c|}{} \\
\hline
\end{tabular}


Rev.08

Title: $\quad$ AS-RUN NEUTRONICS EVALUATION FOR THE GE HITACHI EXPERIMENT IN THE ATR

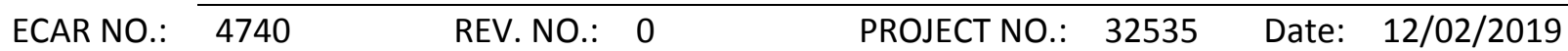

\section{DISCUSSION / ANALYSIS}

MCNP is used to calculate the heating rates, flux, and DPA for GE-HITACHI. ORIGEN2 was used to calculate the decay heat, radionuclide inventory, and radionuclide source term versus cooling time for GE-HITACHI.

\subsection{Neutron/Prompt Gamma Heating and Delayed Fission Product Gamma Heating Normalization Factors}

MCNP reports tally results normalized per source particle. The MCNP Type 6 energy deposition tally results or Type 7 fission energy deposition tally results are used to calculate HGRs. The MCNP Tally Type 6 has units of MeV/g per source particle (fission neutron for prompt neutron, gamma heating, and fission heating). The heating normalization factor (HNF) is defined by Equation (1).

$$
\begin{gathered}
H N F=\left(\frac{2.43 \text { fission neutrons }}{\text { fission }}\right)\left(\frac{\text { fission }}{200 \mathrm{MeV}}\right)\left(\frac{1 \times 10^{6} \mathrm{~W}}{1 \mathrm{MW}}\right) \\
H N F=1.215 \times 10^{4} \frac{\mathrm{fission} \mathrm{neutrons} \cdot \mathrm{W}}{\mathrm{MW} \cdot \mathrm{MeV}}
\end{gathered}
$$

The HGR values are calculated using the MCNP Tally Type 6 results, the HNF, and the ATR core power. Prompt neutron and gamma heating rates (PHRs) are calculated using Equation (2).

$$
\begin{gathered}
\text { PHR }=\left(\text { type } 6 \text { tally } \frac{M e V}{g \cdot \text { fission neutron }}\right)(1.215 \\
\left.\times 104 \frac{\text { fission neutrons } \cdot W}{M W \cdot M e V}\right)(\text { Core Power } M W) \\
P H R=(f 6)(H N F)(\text { Core Power }) \frac{W}{g}
\end{gathered}
$$

MCNP reports tally results normalized per source particle. The heating tallies have units of MeV/g per fission neutron. The MCNP Type 6 energy deposition tally results are used to calculate delayed gamma HGRs. The MCNP Tally Type 6 has units of MeV/g per source particle (per delayed fission product gamma for delayed fission product gamma heating). The delayed gamma heating normalization factor (DNF) is defined by Equation (3) using 8.9603 delayed fission photons per fission. 
Rev.08

Title: $\quad$ AS-RUN NEUTRONICS EVALUATION FOR THE GE HITACHI EXPERIMENT IN THE ATR

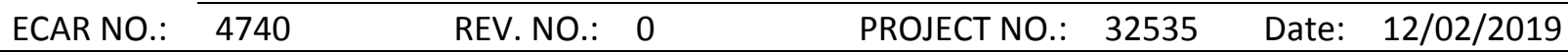

$$
\begin{gathered}
\text { DFN }=\left(\frac{8.9603 \text { delayed photons }}{\text { fission }}\right)\left(\frac{\text { fission }}{200 \mathrm{MeV}}\right)\left(\frac{1 \times 10^{6} \mathrm{~W}}{\mathrm{MW}}\right) \\
D F N=4.480 \times 10^{4} \frac{\mathrm{delayed} \mathrm{photons} \cdot \mathrm{W}}{\mathrm{M} W_{\text {core power }} \cdot \mathrm{MeV}}
\end{gathered}
$$

The HGR values are calculated using the MCNP Tally Type 6 or Type 7 results, the HNF, and the ATR core power. The delayed fission product heating rate (DHR) is calculated using Equation (4).

$$
\begin{gathered}
\text { DHR }=\left(\text { type } 6 \text { tally } \frac{M e V}{g \cdot \text { source photon }}\right)(4.480 \\
\left.\times 10^{4} \frac{\text { delayed photons } \cdot W}{M W \cdot M e V}\right)(\text { Core Power } M W) \\
D H R=(f 6)(D N F)(\text { Core Power }) \frac{W}{g}
\end{gathered}
$$

\subsection{Neutron Flux Normalization Factor}

MCNP reports tally results normalized per source particle. The MCNP Type 4 flux tally results are used to generate neutron flux input values for the ORIGEN2 activation calculations. The MCNP Tally Type 4 (for neutrons) has units of neutrons $/ \mathrm{cm}^{2}$ per source neutron. The neutron flux conversion factor (NFCF) is defined by Equation (5).

$$
\begin{gathered}
\text { NFCF }=\left(\frac{2.43 \text { fission neutrons }}{\text { fission }}\right)\left(\frac{\text { fission }}{200 \mathrm{MeV}}\right)\left(\frac{6.24151 \times 10^{18} \mathrm{MeV}}{M W_{\text {Core Power }} \cdot \mathrm{s}}\right) \\
N F C F=7.583 \times 10^{16} \frac{\mathrm{fission} \text { neutrons }}{\mathrm{M} W_{\text {Core Power }} \cdot \mathrm{s}}
\end{gathered}
$$

The neutron flux values are calculated using the MCNP tally type 4 results, the NFCF, and the ATR core power. The neutron flux is calculated using Equation (6). 
Rev.08

Title: $\quad$ AS-RUN NEUTRONICS EVALUATION FOR THE GE HITACHI EXPERIMENT IN THE ATR

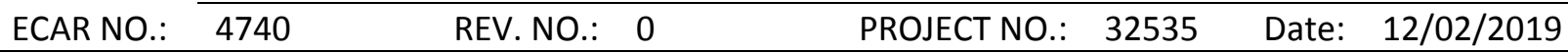

$$
\begin{aligned}
& \phi_{\text {neutron }}=\left(\text { type } 4 \text { tally } \frac{\text { neutrons }}{\mathrm{cm}^{2}-\text { fission neutron }}\right)(7.583 \\
& \left.\quad \times 10^{16} \frac{\text { fission neutrons }}{\mathrm{MW}_{\text {core power }}-\mathrm{s}}\right)(\text { Core Power MW }) \\
& \phi_{\text {neutron }}=(\mathrm{f} 4)\left(7.583 \times 10^{16}\right)(\text { Core Power }) \frac{\text { neutrons }}{\mathrm{cm}^{2}-\mathrm{s}}
\end{aligned}
$$

\subsection{MCNP DPA Calculations}

The DPA rate in a material is estimated by using a tally multiplier card with a standard flux tally in MCNP. The tally multiplier card applied to a flux tally calculates the reaction rate, which is defined as the quantity:

$$
C \int \Phi(E) R_{m}(E) d E
$$

Where,

$$
\begin{array}{lll}
C & = & \text { multiplicative constant } \\
\Phi(E)= & \text { energy dependent flux } \\
R_{m}(E)= & \text { energy dependent reaction rate of interest (ENDF/B-VII damage cross-section) }
\end{array}
$$

The quantity defined in Equation 12 is the total damage energy rate for the material. Assigning the quantity $\eta / 2 E_{d}$ to the constant $C$ in Equation (7) results in:

$$
\text { DPA rate }=C \int \Phi(E) R_{m}(E) d E
$$

Where,

$$
C=\eta / 2 E d
$$

$\int \Phi(E) R m(E) d E$ = total damage energy.

The DPA cross section would be calculated by:

$$
\sigma_{\mathrm{DPA}}=\frac{\int \Phi(E) R_{\mathrm{m}}(\mathrm{E}) \mathrm{dE}}{\int \Phi(E) d E}
$$


TEM-10200-1

$12 / 19 / 17$

ENGINEERING CALCULATIONS AND ANALYSIS

Page 16 of 57

Rev.08

Title: $\quad$ AS-RUN NEUTRONICS EVALUATION FOR THE GE HITACHI EXPERIMENT IN THE ATR

\begin{tabular}{llllllll}
\cline { 2 - 4 } ECAR NO.: & 4740 & REV. NO.: & 0 & PROJECT NO.: & 32535 & Date: & 12/02/2019 \\
\hline
\end{tabular}

The resulting cross section has units of MeV-barns per atom.

Therefore, using the MCNP tallies, the DPA rate is calculated by converting the flux multiplier result to units of $\mathrm{MeV}-\mathrm{cm}^{2}$ per atom, then multiplying by the efficiency ( $\eta$ ) and dividing by 2 times the cutoff energy $\left(E_{d}\right)$ then using the standard tally conversion factors:

$$
D P A \text { rate }=F M n \times \frac{\eta}{2 E_{d}} \times \text { Flux Normalization Factor } \times \text { Core Power }
$$

\section{RESULTS}

\subsection{Heating Results}

Heating rates were generated assuming nominal core power configurations for cycle 162A through 164B [1]. The source power is assumed to be scaled to a nominal south power of $24.3 \mathrm{MW}, 22.0 \mathrm{MW}, 22.5$ MW, and 22.6 MW, for ATR cycle 162A, 162B, 164A, and 164B, respectively. The heating results include prompt neutron and gamma heating, as well as delayed gamma heating. Delayed gamma heating was explicitly calculated using a separate MCNP model. The results for the heat generation rate calculations are presented in Table 6 - Table 7. 
TEM-10200-1

Rev.08

Title: AS-RUN NEUTRONICS EVALUATION FOR THE GE HITACHI EXPERIMENT IN THE ATR

\begin{tabular}{llllllll} 
ECAR NO.: & 4740 & REV. NO.: & 0 & PROJECT NO.: & 32535 & Date: & $12 / 02 / 2019$ \\
\hline
\end{tabular}

Table 6. Pressure Boundary Internal Component, End Cap, and Spacer Heat Generation Rates.

\begin{tabular}{|c|c|c|c|c|}
\hline \multirow{2}{*}{ Component } & $162 A$ & 162B & $164 \mathrm{~A}$ & 164B \\
\hline & $\mathrm{w} / \mathrm{g}$ & $\mathrm{W} / \mathrm{g}$ & $\mathrm{W} / \mathrm{g}$ & $\mathbf{W} / \mathrm{g}$ \\
\hline Upper Al spacer & 1.6 & 1.5 & 1.5 & 1.5 \\
\hline Upper Pressure Boundary Cap & 3.2 & 2.9 & 3.0 & 3.0 \\
\hline Upper YTZP Insulating Disc & 3.5 & 3.1 & 3.2 & 3.2 \\
\hline NE Upper 316 Tensile specimen & 4.0 & 3.9 & 3.7 & 4.0 \\
\hline NE Upper Tensile sleeve & 4.0 & 3.7 & 3.7 & 3.8 \\
\hline SW Upper 316 Tensile specimen & 3.3 & 3.2 & 3.1 & 3.3 \\
\hline SW Upper Tensile sleeve & 3.3 & 3.1 & 3.1 & 3.2 \\
\hline NW Upper 718 Tensile specimen & 4.3 & 3.6 & 4.0 & 3.7 \\
\hline NW Upper Tensile sleeve & 4.0 & 3.6 & 3.7 & 3.7 \\
\hline SE Upper 718 Tensile specimen & 3.6 & 3.0 & 3.3 & 3.1 \\
\hline SE Upper Tensile sleeve & 3.4 & 3.1 & 3.1 & 3.2 \\
\hline Upper Tensile specimen holder annulus & 3.0 & 2.8 & 2.8 & 2.9 \\
\hline Upper Flux Monitor & 3.3 & 3.1 & 3.1 & 3.2 \\
\hline Upper SS316 CT spacer & 3.8 & 3.5 & 3.5 & 3.6 \\
\hline CT 718 specimen 12 & 4.2 & 3.8 & 3.9 & 3.9 \\
\hline CT 316 specimen 11 & 4.1 & 3.6 & 3.8 & 3.7 \\
\hline CT 718 specimen 10 & 4.4 & 4.1 & 4.1 & 4.2 \\
\hline CT 316 specimen 9 & 4.2 & 3.7 & 3.9 & 3.8 \\
\hline CT 718 specimen 8 & 4.6 & 4.1 & 4.3 & 4.2 \\
\hline CT 316 specimen 7 & 4.2 & 3.9 & 3.9 & 4.0 \\
\hline SS Center clamshell & 4.2 & 3.9 & 3.9 & 4.0 \\
\hline Center Flux Monitor & 3.6 & 3.2 & 3.3 & 3.3 \\
\hline CT 718 specimen 6 & 4.6 & 4.2 & 4.3 & 4.3 \\
\hline CT 316 specimen 5 & 4.3 & 3.9 & 4.0 & 4.0 \\
\hline CT 718 specimen 4 & 4.5 & 4.1 & 4.2 & 4.2 \\
\hline CT 316 specimen 3 & 4.2 & 3.9 & 3.9 & 4.0 \\
\hline CT 718 specimen 2 & 4.5 & 4.1 & 4.2 & 4.2 \\
\hline CT 316 specimen 1 & 4.1 & 3.7 & 3.8 & 3.8 \\
\hline Lower SS316 CT spacer & 3.9 & 3.5 & 3.6 & 3.6 \\
\hline Lower Tensile specimen holder annulus & 3.2 & 2.9 & 3.0 & 3.0 \\
\hline Lower Flux Monitor & 3.7 & 3.3 & 3.4 & 3.4 \\
\hline NE Lower 316 Tensile specimen & 4.1 & 4.0 & 3.8 & 4.1 \\
\hline NE Lower Tensile sleeve & 4.2 & 3.8 & 3.9 & 3.9 \\
\hline SW Lower 316 Tensile specimen & 3.5 & 3.3 & 3.2 & 3.4 \\
\hline SW Lower Tensile sleeve & 3.5 & 3.2 & 3.2 & 3.3 \\
\hline NW Lower 718 Tensile specimen & 4.5 & 3.8 & 4.2 & 3.9 \\
\hline NW Lower Tensile sleeve & 4.1 & 3.7 & 3.8 & 3.8 \\
\hline
\end{tabular}


Rev.08

Title: AS-RUN NEUTRONICS EVALUATION FOR THE GE HITACHI EXPERIMENT IN THE ATR

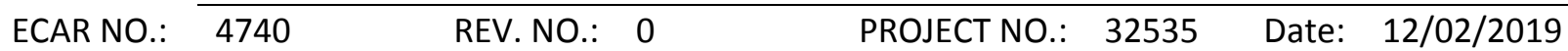

\begin{tabular}{|c|c|c|c|c|}
\hline \multirow{2}{*}{ Component } & $\mathbf{1 6 2 A}$ & $\mathbf{1 6 2 B}$ & $\mathbf{1 6 4 A}$ & $\mathbf{1 6 4 B}$ \\
\cline { 2 - 5 } & $\mathbf{W} / \mathbf{g}$ & $\mathbf{W} / \mathbf{g}$ & $\mathbf{W} / \mathbf{g}$ & $\mathbf{W / g}$ \\
\hline SE Lower 718 Tensile specimen & 3.8 & 3.2 & 3.5 & 3.3 \\
\hline SE Lower Tensile sleeve & 3.6 & 3.2 & 3.3 & 3.3 \\
\hline Lower YTZP insulating disc & 3.7 & 3.3 & 3.4 & 3.4 \\
\hline Lower Pressure Boundary Cap & 3.3 & 3.0 & 3.1 & 3.1 \\
\hline Lower Al spacer & 2.0 & 1.7 & 1.8 & 1.7 \\
\hline
\end{tabular}

Table 7. Beryllium Surrounding B-11 Position Heat Generation Rates.

\begin{tabular}{|c|c|c|c|c|c|c|}
\hline \multirow[t]{2}{*}{ Component } & $162 A$ & 162B & 164A & 164B & $\begin{array}{c}\text { Upper } \\
\text { Elevation }\end{array}$ & $\begin{array}{c}\text { Lower } \\
\text { Elevation }\end{array}$ \\
\hline & $\mathrm{W} / \mathrm{g}$ & $\mathrm{W} / \mathrm{g}$ & $\mathrm{W} / \mathrm{g}$ & $\mathrm{W} / \mathrm{g}$ & $\mathrm{cm}$ & $\mathrm{cm}$ \\
\hline Segment of Beryllium around B-11 & 1.0 & 0.9 & 0.9 & 0.9 & 2.54 & -2.54 \\
\hline Segment of Beryllium around B-11 & 1.3 & 1.2 & 1.2 & 1.2 & 7.62 & 2.54 \\
\hline Segment of Beryllium around B-11 & 1.7 & 1.5 & 1.6 & 1.5 & 12.70 & 7.62 \\
\hline Segment of Beryllium around B-11 & 2.0 & 1.8 & 1.8 & 1.8 & 17.78 & 12.70 \\
\hline Segment of Beryllium around B-11 & 2.3 & 2.1 & 2.1 & 2.2 & 22.86 & 17.78 \\
\hline Segment of Beryllium around B-11 & 2.6 & 2.3 & 2.4 & 2.4 & 27.94 & 22.86 \\
\hline Segment of Beryllium around B-11 & 2.8 & 2.5 & 2.6 & 2.6 & 33.02 & 27.94 \\
\hline Segment of Beryllium around B-11 & 3.0 & 2.8 & 2.8 & 2.9 & 38.10 & 33.02 \\
\hline Segment of Beryllium around B-11 & 3.2 & 2.9 & 3.0 & 3.0 & 43.18 & 38.10 \\
\hline Segment of Beryllium around B-11 & 3.4 & 3.0 & 3.1 & 3.1 & 48.26 & 43.18 \\
\hline Segment of Beryllium around B-11 & 3.5 & 3.2 & 3.2 & 3.3 & 53.34 & 48.26 \\
\hline Segment of Beryllium around B-11 & 3.5 & 3.2 & 3.2 & 3.3 & 58.42 & 53.34 \\
\hline Segment of Beryllium around B-11 & 3.5 & 3.2 & 3.2 & 3.3 & 63.50 & 58.42 \\
\hline Segment of Beryllium around B-11 & 3.5 & 3.1 & 3.2 & 3.2 & 68.58 & 63.50 \\
\hline Segment of Beryllium around B-11 & 3.4 & 3.0 & 3.1 & 3.1 & 73.66 & 68.58 \\
\hline Segment of Beryllium around B-11 & 3.3 & 2.9 & 3.1 & 3.0 & 78.74 & 73.66 \\
\hline Segment of Beryllium around B-11 & 3.0 & 2.8 & 2.8 & 2.9 & 83.82 & 78.74 \\
\hline Segment of Beryllium around B-11 & 2.9 & 2.7 & 2.7 & 2.8 & 88.90 & 83.82 \\
\hline Segment of Beryllium around B-11 & 2.6 & 2.4 & 2.4 & 2.5 & 93.98 & 88.90 \\
\hline Segment of Beryllium around B-11 & 2.3 & 2.1 & 2.1 & 2.2 & 99.06 & 93.98 \\
\hline Segment of Beryllium around B-11 & 2.1 & 1.8 & 1.9 & 1.8 & 104.14 & 99.06 \\
\hline Segment of Beryllium around B-11 & 1.7 & 1.6 & 1.6 & 1.6 & 109.22 & 104.14 \\
\hline Segment of Beryllium around B-11 & 1.3 & 1.2 & 1.2 & 1.2 & 114.30 & 109.22 \\
\hline Segment of Beryllium around B-11 & 1.1 & 1.0 & 1.0 & 1.0 & 119.38 & 114.30 \\
\hline Segment of Beryllium around B-11 & 0.7 & 0.6 & 0.6 & 0.6 & 127.00 & 119.38 \\
\hline
\end{tabular}


TEM-10200-1

Rev.08

Title: AS-RUN NEUTRONICS EVALUATION FOR THE GE HITACHI EXPERIMENT IN THE ATR

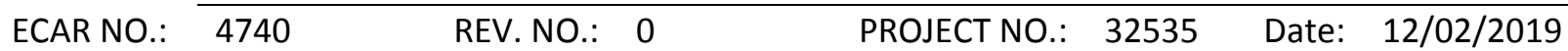

Table 8. Water Annulus Outside Basket Heat Generation Rates.

\begin{tabular}{|c|c|c|c|c|c|c|}
\hline Component & $162 A$ & 162B & 164A & 164B & $\begin{array}{c}\text { Upper } \\
\text { Elevation }\end{array}$ & $\begin{array}{c}\text { Lower } \\
\text { Elevation }\end{array}$ \\
\hline & $\mathbf{w} / \mathrm{g}$ & $\mathrm{w} / \mathrm{g}$ & $\mathrm{w} / \mathrm{g}$ & $\mathrm{w} / \mathrm{g}$ & $\mathbf{c m}$ & $\mathrm{cm}$ \\
\hline Segment of Water annulus outside basket & 1.5 & 1.4 & 1.4 & 1.4 & 2.54 & -2.54 \\
\hline Segment of Water annulus outside basket & 2.2 & 2.0 & 2.0 & 2.1 & 7.62 & 2.54 \\
\hline Segment of Water annulus outside basket & 2.8 & 2.5 & 2.6 & 2.6 & 12.70 & 7.62 \\
\hline Segment of Water annulus outside basket & 3.3 & 3.0 & 3.1 & 3.1 & 17.78 & 12.70 \\
\hline Segment of Water annulus outside basket & 3.9 & 3.6 & 3.6 & 3.7 & 22.86 & 17.78 \\
\hline Segment of Water annulus outside basket & 4.3 & 3.9 & 4.0 & 4.0 & 27.94 & 22.86 \\
\hline Segment of Water annulus outside basket & 4.8 & 4.4 & 4.4 & 4.5 & 33.02 & 27.94 \\
\hline Segment of Water annulus outside basket & 5.2 & 4.7 & 4.8 & 4.8 & 38.10 & 33.02 \\
\hline Segment of Water annulus outside basket & 5.4 & 4.9 & 5.0 & 5.0 & 43.18 & 38.10 \\
\hline Segment of Water annulus outside basket & 5.7 & 5.2 & 5.3 & 5.3 & 48.26 & 43.18 \\
\hline Segment of Water annulus outside basket & 5.9 & 5.3 & 5.5 & 5.4 & 53.34 & 48.26 \\
\hline Segment of Water annulus outside basket & 6.0 & 5.4 & 5.5 & 5.5 & 58.42 & 53.34 \\
\hline Segment of Water annulus outside basket & 5.9 & 5.4 & 5.5 & 5.5 & 63.50 & 58.42 \\
\hline Segment of Water annulus outside basket & 5.9 & 5.4 & 5.5 & 5.5 & 68.58 & 63.50 \\
\hline Segment of Water annulus outside basket & 5.8 & 5.2 & 5.4 & 5.3 & 73.66 & 68.58 \\
\hline Segment of Water annulus outside basket & 5.5 & 4.9 & 5.1 & 5.0 & 78.74 & 73.66 \\
\hline Segment of Water annulus outside basket & 5.2 & 4.7 & 4.8 & 4.8 & 83.82 & 78.74 \\
\hline Segment of Water annulus outside basket & 4.8 & 4.4 & 4.4 & 4.5 & 88.90 & 83.82 \\
\hline Segment of Water annulus outside basket & 4.4 & 4.0 & 4.1 & 4.1 & 93.98 & 88.90 \\
\hline Segment of Water annulus outside basket & 3.9 & 3.6 & 3.6 & 3.7 & 99.06 & 93.98 \\
\hline Segment of Water annulus outside basket & 3.5 & 3.1 & 3.2 & 3.2 & 104.14 & 99.06 \\
\hline Segment of Water annulus outside basket & 2.9 & 2.7 & 2.7 & 2.8 & 109.22 & 104.14 \\
\hline Segment of Water annulus outside basket & 2.3 & 2.1 & 2.1 & 2.2 & 114.30 & 109.22 \\
\hline Segment of Water annulus outside basket & 1.7 & 1.6 & 1.6 & 1.6 & 119.38 & 114.30 \\
\hline Segment of Water annulus outside basket & 1.0 & 0.9 & 0.9 & 0.9 & 127.00 & 119.38 \\
\hline
\end{tabular}

Table 9. Basket Heat Generation Rates.

\begin{tabular}{|c|c|c|c|c|c|c|}
\hline \multirow{2}{*}{ Component } & 162A & 162B & 164A & 164B & $\begin{array}{c}\text { Upper } \\
\text { Elevation }\end{array}$ & $\begin{array}{c}\text { Lower } \\
\text { Elevation }\end{array}$ \\
\cline { 2 - 7 } & $\mathbf{W / g}$ & $\mathbf{W / g}$ & $\mathbf{W} / \mathbf{g}$ & $\mathbf{W} / \mathbf{g}$ & $\mathbf{c m}$ & $\mathbf{c m}$ \\
\hline Segment of Al basket & 1.1 & 1.0 & 1.0 & 1.0 & 2.54 & -2.54 \\
\hline Segment of Al basket & 1.5 & 1.3 & 1.4 & 1.3 & 7.62 & 2.54 \\
\hline Segment of Al basket & 1.8 & 1.6 & 1.7 & 1.6 & 12.70 & 7.62 \\
\hline Segment of Al basket & 2.1 & 2.0 & 1.9 & 2.1 & 17.78 & 12.70 \\
\hline Segment of Al basket & 2.5 & 2.3 & 2.3 & 2.4 & 22.86 & 17.78 \\
\hline Segment of Al basket & 2.8 & 2.5 & 2.6 & 2.6 & 27.94 & 22.86 \\
\hline
\end{tabular}


TEM-10200-1

Rev.08

Title: AS-RUN NEUTRONICS EVALUATION FOR THE GE HITACHI EXPERIMENT IN THE ATR

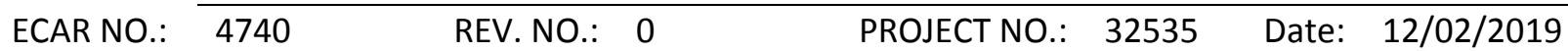

\begin{tabular}{|c|c|c|c|c|c|c|}
\hline \multirow[t]{2}{*}{ Component } & $162 A$ & 162B & $164 A$ & 164B & $\begin{array}{c}\text { Upper } \\
\text { Elevation }\end{array}$ & $\begin{array}{c}\text { Lower } \\
\text { Elevation }\end{array}$ \\
\hline & $\mathrm{W} / \mathrm{g}$ & $\mathrm{W} / \mathrm{g}$ & $\mathrm{W} / \mathrm{g}$ & $W / g$ & $\mathrm{~cm}$ & cm \\
\hline Segment of Al basket & 3.1 & 2.7 & 2.9 & 2.8 & 33.02 & 27.94 \\
\hline Segment of Al basket & 3.3 & 3.0 & 3.1 & 3.1 & 38.10 & 33.02 \\
\hline Segment of Al basket & 3.5 & 3.1 & 3.2 & 3.2 & 43.18 & 38.10 \\
\hline Segment of Al basket & 3.6 & 3.2 & 3.3 & 3.3 & 48.26 & 43.18 \\
\hline Segment of Al basket & 3.7 & 3.4 & 3.4 & 3.5 & 53.34 & 48.26 \\
\hline Segment of Al basket & 3.8 & 3.5 & 3.5 & 3.6 & 58.42 & 53.34 \\
\hline Segment of Al basket & 3.8 & 3.5 & 3.5 & 3.6 & 63.50 & 58.42 \\
\hline Segment of Al basket & 3.7 & 3.4 & 3.4 & 3.5 & 68.58 & 63.50 \\
\hline Segment of Al basket & 3.6 & 3.4 & 3.3 & 3.5 & 73.66 & 68.58 \\
\hline Segment of Al basket & 3.5 & 3.1 & 3.2 & 3.2 & 78.74 & 73.66 \\
\hline Segment of Al basket & 3.3 & 3.0 & 3.1 & 3.1 & 83.82 & 78.74 \\
\hline Segment of Al basket & 3.1 & 2.9 & 2.9 & 3.0 & 88.90 & 83.82 \\
\hline Segment of Al basket & 2.9 & 2.6 & 2.7 & 2.7 & 93.98 & 88.90 \\
\hline Segment of Al basket & 2.5 & 2.4 & 2.3 & 2.5 & 99.06 & 93.98 \\
\hline Segment of Al basket & 2.2 & 2.0 & 2.0 & 2.1 & 104.14 & 99.06 \\
\hline Segment of Al basket & 1.9 & 1.7 & 1.8 & 1.7 & 109.22 & 104.14 \\
\hline Segment of Al basket & 1.5 & 1.4 & 1.4 & 1.4 & 114.30 & 109.22 \\
\hline Segment of Al basket & 1.1 & 1.0 & 1.0 & 1.0 & 119.38 & 114.30 \\
\hline Segment of Al basket & 0.7 & 0.6 & 0.6 & 0.6 & 127.00 & 119.38 \\
\hline
\end{tabular}

Table 10. Water Annulus Inside Basket Heat Generation Rates.

\begin{tabular}{|c|c|c|c|c|c|c|}
\hline \multirow{2}{*}{ Component } & 162A & 162B & 164A & 164B & $\begin{array}{c}\text { Upper } \\
\text { Elevation }\end{array}$ & $\begin{array}{c}\text { Lower } \\
\text { Elevation }\end{array}$ \\
\cline { 2 - 7 } & $\mathbf{W / g}$ & $\mathbf{W / g}$ & $\mathbf{W} / \mathbf{g}$ & $\mathbf{W} / \mathbf{g}$ & $\mathbf{c m}$ & $\mathbf{c m}$ \\
\hline Segment of Water annulus inside basket & 1.5 & 1.4 & 1.4 & 1.4 & 2.54 & -2.54 \\
\hline Segment of Water annulus inside basket & 2.2 & 2.0 & 2.0 & 2.1 & 7.62 & 2.54 \\
\hline Segment of Water annulus inside basket & 2.8 & 2.5 & 2.6 & 2.6 & 12.70 & 7.62 \\
\hline Segment of Water annulus inside basket & 3.3 & 3.0 & 3.1 & 3.1 & 17.78 & 12.70 \\
\hline Segment of Water annulus inside basket & 3.9 & 3.6 & 3.6 & 3.7 & 22.86 & 17.78 \\
\hline Segment of Water annulus inside basket & 4.4 & 3.9 & 4.1 & 4.0 & 27.94 & 22.86 \\
\hline Segment of Water annulus inside basket & 4.8 & 4.3 & 4.4 & 4.4 & 33.02 & 27.94 \\
\hline Segment of Water annulus inside basket & 5.1 & 4.7 & 4.7 & 4.8 & 38.10 & 33.02 \\
\hline Segment of Water annulus inside basket & 5.4 & 4.9 & 5.0 & 5.0 & 43.18 & 38.10 \\
\hline Segment of Water annulus inside basket & 5.7 & 5.1 & 5.3 & 5.2 & 48.26 & 43.18 \\
\hline Segment of Water annulus inside basket & 5.8 & 5.3 & 5.4 & 5.4 & 53.34 & 48.26 \\
\hline Segment of Water annulus inside basket & 5.9 & 5.4 & 5.5 & 5.5 & 58.42 & 53.34 \\
\hline Segment of Water annulus inside basket & 6.0 & 5.4 & 5.5 & 5.5 & 63.50 & 58.42 \\
\hline
\end{tabular}


TEM-10200-1

Rev.08

Title: AS-RUN NEUTRONICS EVALUATION FOR THE GE HITACHI EXPERIMENT IN THE ATR

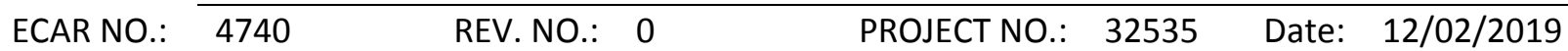

\begin{tabular}{|c|c|c|c|c|c|c|}
\hline \multirow[t]{2}{*}{ Component } & $162 A$ & 162B & 164A & 164B & $\begin{array}{c}\text { Upper } \\
\text { Elevation }\end{array}$ & $\begin{array}{c}\text { Lower } \\
\text { Elevation }\end{array}$ \\
\hline & $\mathrm{W} / \mathrm{g}$ & $\mathbf{W} / \mathrm{g}$ & $\mathbf{W} / \mathrm{g}$ & $\mathbf{W} / g$ & $\mathbf{c m}$ & $\mathbf{c m}$ \\
\hline Segment of Water annulus inside basket & 5.8 & 5.4 & 5.4 & 5.5 & 68.58 & 63.50 \\
\hline Segment of Water annulus inside basket & 5.7 & 5.2 & 5.3 & 5.3 & 73.66 & 68.58 \\
\hline Segment of Water annulus inside basket & 5.5 & 4.9 & 5.1 & 5.0 & 78.74 & 73.66 \\
\hline Segment of Water annulus inside basket & 5.2 & 4.7 & 4.8 & 4.8 & 83.82 & 78.74 \\
\hline Segment of Water annulus inside basket & 4.8 & 4.4 & 4.4 & 4.5 & 88.90 & 83.82 \\
\hline Segment of Water annulus inside basket & 4.4 & 4.0 & 4.1 & 4.1 & 93.98 & 88.90 \\
\hline Segment of Water annulus inside basket & 4.0 & 3.6 & 3.7 & 3.7 & 99.06 & 93.98 \\
\hline Segment of Water annulus inside basket & 3.4 & 3.1 & 3.1 & 3.2 & 104.14 & 99.06 \\
\hline Segment of Water annulus inside basket & 2.9 & 2.6 & 2.7 & 2.7 & 109.22 & 104.14 \\
\hline Segment of Water annulus inside basket & 2.3 & 2.1 & 2.1 & 2.2 & 114.30 & 109.22 \\
\hline Segment of Water annulus inside basket & 1.7 & 1.5 & 1.6 & 1.5 & 119.38 & 114.30 \\
\hline Segment of Water annulus inside basket & 1.0 & 0.9 & 0.9 & 0.9 & 127.00 & 119.38 \\
\hline
\end{tabular}

Table 11. Pressure Boundary Heat Generation rates.

\begin{tabular}{|c|c|c|c|c|c|c|}
\hline \multirow{2}{*}{ Component } & 162A & 162B & 164A & 164B & $\begin{array}{c}\text { Upper } \\
\text { Elevation }\end{array}$ & $\begin{array}{c}\text { Lower } \\
\text { Elevation }\end{array}$ \\
\cline { 2 - 7 } & $\mathbf{W / g}$ & $\mathbf{W / g}$ & $\mathbf{W / g}$ & $\mathbf{W / g}$ & $\mathbf{c m}$ & $\mathbf{c m}$ \\
\hline Segment of pressure boundary & 1.5 & 1.4 & 1.4 & 1.4 & 2.54 & -2.54 \\
\hline Segment of pressure boundary & 1.9 & 1.7 & 1.8 & 1.7 & 7.62 & 2.54 \\
\hline Segment of pressure boundary & 2.3 & 2.2 & 2.1 & 2.3 & 12.70 & 7.62 \\
\hline Segment of pressure boundary & 2.8 & 2.5 & 2.6 & 2.6 & 17.78 & 12.70 \\
\hline Segment of pressure boundary & 3.2 & 2.9 & 3.0 & 3.0 & 22.86 & 17.78 \\
\hline Segment of pressure boundary & 3.6 & 3.2 & 3.3 & 3.3 & 27.94 & 22.86 \\
\hline Segment of pressure boundary & 3.8 & 3.4 & 3.5 & 3.5 & 33.02 & 27.94 \\
\hline Segment of pressure boundary & 4.2 & 3.8 & 3.9 & 3.9 & 38.10 & 33.02 \\
\hline Segment of pressure boundary & 4.3 & 3.9 & 4.0 & 4.0 & 43.18 & 38.10 \\
\hline Segment of pressure boundary & 4.5 & 4.2 & 4.2 & 4.3 & 48.26 & 43.18 \\
\hline Segment of pressure boundary & 4.7 & 4.3 & 4.3 & 4.4 & 53.34 & 48.26 \\
\hline Segment of pressure boundary & 4.8 & 4.3 & 4.4 & 4.4 & 58.42 & 53.34 \\
\hline Segment of pressure boundary & 4.8 & 4.3 & 4.4 & 4.4 & 63.50 & 58.42 \\
\hline Segment of pressure boundary & 4.7 & 4.3 & 4.3 & 4.4 & 68.58 & 63.50 \\
\hline Segment of pressure boundary & 4.6 & 4.2 & 4.3 & 4.3 & 73.66 & 68.58 \\
\hline Segment of pressure boundary & 4.4 & 3.9 & 4.1 & 4.0 & 78.74 & 73.66 \\
\hline Segment of pressure boundary & 4.2 & 3.8 & 3.9 & 3.9 & 83.82 & 78.74 \\
\hline Segment of pressure boundary & 3.9 & 3.6 & 3.6 & 3.7 & 88.90 & 83.82 \\
\hline Segment of pressure boundary & 3.5 & 3.2 & 3.2 & 3.3 & 93.98 & 88.90 \\
\hline Segment of pressure boundary & 3.3 & 3.0 & 3.1 & 3.1 & 99.06 & 93.98 \\
\hline
\end{tabular}


TEM-10200-1

$12 / 19 / 17$

ENGINEERING CALCULATIONS AND ANALYSIS

Page 22 of $\mathbf{5 7}$

Rev.08

Title: $\quad$ AS-RUN NEUTRONICS EVALUATION FOR THE GE HITACHI EXPERIMENT IN THE ATR

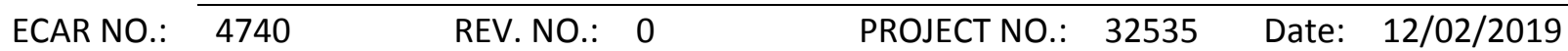

\begin{tabular}{|c|c|c|c|c|c|c|}
\hline \multirow[t]{2}{*}{ Component } & $162 A$ & 162B & 164A & 164B & $\begin{array}{c}\text { Upper } \\
\text { Elevation }\end{array}$ & $\begin{array}{c}\text { Lower } \\
\text { Elevation }\end{array}$ \\
\hline & W/g & W/g & $\mathrm{W} / \mathrm{g}$ & $\mathbf{W} / \mathrm{g}$ & $\mathrm{cm}$ & $\mathbf{c m}$ \\
\hline Segment of pressure boundary & 2.8 & 2.6 & 2.6 & 2.7 & 104.14 & 99.06 \\
\hline Segment of pressure boundary & 2.4 & 2.2 & 2.2 & 2.3 & 109.22 & 104.14 \\
\hline Segment of pressure boundary & 1.9 & 1.7 & 1.8 & 1.7 & 114.30 & 109.22 \\
\hline Segment of pressure boundary & 1.4 & 1.3 & 1.3 & 1.3 & 119.38 & 114.30 \\
\hline Segment of pressure boundary & 0.9 & 0.9 & 0.8 & 0.9 & 127.00 & 119.38 \\
\hline
\end{tabular}

Table 12. CT Specimen Holder Heat Generation Rates.

\begin{tabular}{|c|c|c|c|c|c|c|}
\hline \multirow[t]{2}{*}{ Component } & $162 A$ & 162B & 164A & 164B & $\begin{array}{c}\text { Upper } \\
\text { Elevation }\end{array}$ & $\begin{array}{c}\text { Lower } \\
\text { Elevation }\end{array}$ \\
\hline & $\mathrm{W} / \mathrm{g}$ & $\mathbf{W} / \mathrm{g}$ & $\mathbf{W} / \mathrm{g}$ & $\mathrm{W} / \mathrm{g}$ & $\mathbf{c m}$ & cm \\
\hline Segment of CT specimen holder & 3.4 & 3.2 & 3.1 & 3.3 & 47.12 & 44.48 \\
\hline Segment of CT specimen holder & 3.5 & 3.2 & 3.2 & 3.3 & 49.76 & 47.12 \\
\hline Segment of CT specimen holder & 3.6 & 3.2 & 3.3 & 3.3 & 52.40 & 49.76 \\
\hline Segment of CT specimen holder & 3.6 & 3.2 & 3.3 & 3.3 & 55.04 & 52.40 \\
\hline Segment of CT specimen holder & 3.6 & 3.3 & 3.3 & 3.4 & 57.68 & 55.04 \\
\hline Segment of CT specimen holder & 3.6 & 3.3 & 3.3 & 3.4 & 60.33 & 57.68 \\
\hline Segment of CT specimen holder & 3.5 & 3.3 & 3.2 & 3.4 & 61.60 & 60.33 \\
\hline Segment of CT specimen holder & 3.5 & 3.2 & 3.2 & 3.3 & 64.24 & 61.60 \\
\hline Segment of CT specimen holder & 3.5 & 3.3 & 3.2 & 3.4 & 66.88 & 64.24 \\
\hline Segment of CT specimen holder & 3.5 & 3.2 & 3.2 & 3.3 & 69.52 & 66.88 \\
\hline Segment of CT specimen holder & 3.5 & 3.1 & 3.2 & 3.2 & 72.16 & 69.52 \\
\hline Segment of CT specimen holder & 3.5 & 3.1 & 3.2 & 3.2 & 74.80 & 72.16 \\
\hline Segment of CT specimen holder & 3.3 & 3.0 & 3.1 & 3.1 & 77.44 & 74.80 \\
\hline
\end{tabular}

\subsection{DPA Results}


TEM-10200-1

Rev.08

Title: $\quad$ AS-RUN NEUTRONICS EVALUATION FOR THE GE HITACHI EXPERIMENT IN THE ATR

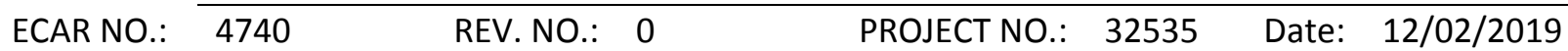

The projected cumulative DPA was calculated for the GE-HITACHI sample specimen. The efficiency was assumed to be $80 \%$. The cutoff energy for 316 stainless steel and Inconel-718 used in the DPA calculation was $4.02 \times 10^{-5} \mathrm{MeV}$ and $4.02 \times 10^{-5} \mathrm{MeV}$, respectively (see ECAR-3569 [14]). Table 13 reports the projected DPA for the GE-HITACHI experiment after four ATR cycles of irradiation, 219 EFPDs.

Table 13. DPA for GE-HITACHI Samples.

\begin{tabular}{|c|c|c|c|c|c|c|}
\hline \multirow{4}{*}{ Material } & Cycle: & 162A & 162B & 164A & 164B & Total \\
\cline { 2 - 7 } & EFPDs: & $\mathbf{6 1 . 9 0}$ & $\mathbf{3 8 . 5 1}$ & $\mathbf{5 4 . 9 1}$ & $\mathbf{6 4 . 0 6}$ & $\mathbf{2 1 9 . 3 8}$ \\
\hline \multirow{5}{*}{$\begin{array}{c}\text { SS316L } \\
\text { Samples }\end{array}$} & N Tensile Specimen & 0.22 & 0.13 & 0.18 & 0.22 & 0.74 \\
\cline { 2 - 7 } & S Tensile Specimen & 0.25 & 0.14 & 0.21 & 0.25 & 0.85 \\
\cline { 2 - 7 } & CT Specimen 11 - 316L & 0.26 & 0.15 & 0.21 & 0.25 & 0.87 \\
\cline { 2 - 7 } & CT Specimen 9 - 316L & 0.26 & 0.15 & 0.22 & 0.25 & 0.89 \\
\cline { 2 - 7 } & CT Specimen 7 - 316L & 0.27 & 0.15 & 0.23 & 0.26 & 0.92 \\
\cline { 2 - 7 } & CT Specimen 5 - 316L & 0.27 & 0.15 & 0.22 & 0.26 & 0.91 \\
\cline { 2 - 7 } & CT Specimen 3 - 316L & 0.27 & 0.15 & 0.22 & 0.26 & 0.90 \\
\cline { 2 - 7 } & CT Specimen 1 - 316L & 0.26 & 0.14 & 0.21 & 0.25 & 0.85 \\
\cline { 2 - 7 } & NE Tensile Specimen & 0.26 & 0.15 & 0.22 & 0.25 & 0.88 \\
\cline { 2 - 7 } & SW Tensile Specimen & 0.23 & 0.13 & 0.19 & 0.22 & 0.78 \\
\cline { 2 - 7 } & E Tensile Specimen & 0.29 & 0.17 & 0.24 & 0.29 & 0.99 \\
\cline { 2 - 7 } Inconel- & W Tensile Specimen & 0.26 & 0.14 & 0.21 & 0.25 & 0.86 \\
\cline { 2 - 7 } 718 & CT Specimen 12 - 718 & 0.30 & 0.17 & 0.24 & 0.29 & 0.99 \\
\cline { 2 - 7 } & CT Specimen 10 - 718 & 0.30 & 0.17 & 0.25 & 0.29 & 1.01 \\
\cline { 2 - 7 } & CT Specimen 8 - 718 & 0.31 & 0.18 & 0.26 & 0.30 & 1.05 \\
\cline { 2 - 7 } & CT Specimen 6 - 718 & 0.32 & 0.18 & 0.26 & 0.31 & 1.07 \\
\cline { 2 - 7 } & CT Specimen 4 - 718 & 0.32 & 0.18 & 0.26 & 0.30 & 1.06 \\
\cline { 2 - 7 } & CT Specimen 2 - 718 & 0.31 & 0.17 & 0.25 & 0.29 & 1.02 \\
\cline { 2 - 7 } & NW Tensile Specimen & 0.31 & 0.17 & 0.25 & 0.30 & 1.03 \\
\cline { 2 - 7 } & SE Tensile Specimen & 0.27 & 0.15 & 0.22 & 0.26 & 0.90 \\
\hline
\end{tabular}

Table 14. DPA for all SS316L and Inconel 718 Specimens after four cycles of irradiation.

\begin{tabular}{|c|c|c|c|c|}
\hline Material & Total EFPD & $\min$ & average & $\max$ \\
\hline SS316L & \multirow{2}{*}{219} & 0.74 & 0.86 & 0.92 \\
\cline { 1 - 4 } Inconel 718 & & 0.86 & 1.00 & 1.07 \\
\hline
\end{tabular}


TEM-10200-1

$12 / 19 / 17$

ENGINEERING CALCULATIONS AND ANALYSIS

Page $\mathbf{2 4}$ of $\mathbf{5 7}$

Rev.08

Title: $\quad$ AS-RUN NEUTRONICS EVALUATION FOR THE GE HITACHI EXPERIMENT IN THE ATR

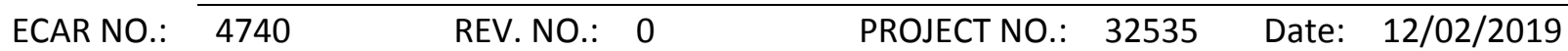

\subsection{Decay Heat}

The decay heat from the as run radionuclide inventory was calculated for the GE HITACHI experiment using ORIGEN2. The peak neutron flux, irradiation time of 219 EFPDs, and experiment component compositional data were used to calculate the as-run volumetric decay heat for each material and the results are reported in Table 15.

Table 15. Volumetric Decay Heat for Experiment Materials.

\begin{tabular}{|c|c|c|c|c|c|c|c|c|c|c|}
\hline & EOI & 30.0MI & $1.0 \mathrm{D}$ & $7.0 \mathrm{D}$ & $30.0 \mathrm{D}$ & $60.0 \mathrm{D}$ & $90.0 \mathrm{D}$ & 120.0D & 200.0D & $360.0 \mathrm{D}$ \\
\hline Material & $\mathrm{W} / \mathrm{cc}$ & W/cc & W/cc & W/cc & $\mathrm{W} / \mathrm{cc}$ & W/cc & W/cc & W/cc & W/cc & $\mathrm{W} / \mathrm{cc}$ \\
\hline SS316L & 0.61 & 0.53 & 0.02 & 0.01 & 0.01 & 0.01 & 0.00 & 0.00 & 0.00 & 0.00 \\
\hline Inconel 718 & 0.28 & 0.24 & 0.12 & 0.11 & 0.09 & 0.08 & 0.08 & 0.07 & 0.07 & 0.06 \\
\hline Aluminum & 0.37 & 0.02 & 0.00 & 0.00 & 0.00 & 0.00 & 0.00 & 0.00 & 0.00 & 0.00 \\
\hline YTZP Zirconia & 0.24 & 0.09 & 0.06 & 0.04 & 0.02 & 0.02 & 0.01 & 0.01 & 0.00 & 0.00 \\
\hline
\end{tabular}

\subsection{Radionuclide Source Term}

The source term for the GE-HITACHI experiment was evaluated using the neutron flux for the experiment in the B-11 position. The peak MCNP calculated neutron flux for the entire experiment irradiation was $5.28 \times 10^{14} \mathrm{n} / \mathrm{cm}^{2} \cdot \mathrm{s}$. This flux value was used to calculate all radiological source terms and the decay heat generation rates for the GE-HITACHI experiment.

Table 16 provides the summary of the radionuclide activity (in Curies) for the entire GE-HITACHI experiment immediately after shutdown and for an additional 360 days after. 
TEM-10200-1 $12 / 19 / 17$

Rev.08

Title: AS-RUN NEUTRONICS EVALUATION FOR THE GE HITACHI EXPERIMENT IN THE ATR

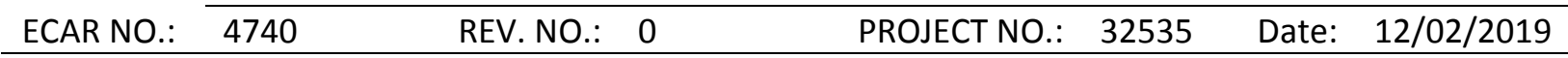

Table 16. Radionuclide source term for the GE-HITACHI experiment after 219 days of irradiation (Ci).

\begin{tabular}{|c|c|c|c|c|c|c|c|c|c|c|}
\hline \multirow{2}{*}{ Isotope } & EOI & $30.0 \mathrm{MI}$ & 1.0D & 7.0D & 30.0D & 60.0D & 90.0D & 120.0D & 200.0D & $360.0 \mathrm{D}$ \\
\hline & $\mathrm{Ci}$ & $\mathrm{Ci}$ & $\mathrm{Ci}$ & $\mathrm{Ci}$ & $\mathrm{Ci}$ & $\mathbf{C i}$ & $\mathrm{Ci}$ & $\mathrm{Ci}$ & $\mathbf{C i}$ & $\mathrm{Ci}$ \\
\hline H 3 & $3.38 \mathrm{E}-07$ & $3.38 \mathrm{E}-07$ & $3.38 \mathrm{E}-07$ & $3.37 \mathrm{E}-07$ & $3.36 \mathrm{E}-07$ & $3.35 \mathrm{E}-07$ & $3.33 \mathrm{E}-07$ & $3.32 \mathrm{E}-07$ & $3.28 \mathrm{E}-07$ & $3.20 \mathrm{E}-07$ \\
\hline H 4 & $3.42 \mathrm{E}-02$ & $0.00 \mathrm{E}+00$ & $0.00 \mathrm{E}+00$ & $0.00 \mathrm{E}+00$ & $0.00 \mathrm{E}+00$ & $0.00 \mathrm{E}+00$ & $0.00 \mathrm{E}+00$ & $0.00 \mathrm{E}+00$ & $0.00 \mathrm{E}+00$ & $0.00 \mathrm{E}+0 \mathrm{C}$ \\
\hline HE 6 & $8.77 \mathrm{E}-05$ & $0.00 \mathrm{E}+00$ & $0.00 \mathrm{E}+00$ & $0.00 \mathrm{E}+00$ & $0.00 \mathrm{E}+00$ & $0.00 \mathrm{E}+00$ & $0.00 \mathrm{E}+00$ & $0.00 \mathrm{E}+00$ & $0.00 \mathrm{E}+00$ & $0.00 \mathrm{E}+0 \mathrm{C}$ \\
\hline LI 8 & 1.37E-02 & $0.00 \mathrm{E}+00$ & $0.00 \mathrm{E}+00$ & $0.00 \mathrm{E}+00$ & $0.00 \mathrm{E}+00$ & $0.00 \mathrm{E}+00$ & $0.00 \mathrm{E}+00$ & $0.00 \mathrm{E}+00$ & $0.00 \mathrm{E}+00$ & $0.00 \mathrm{E}+0 \mathrm{C}$ \\
\hline BE 8 & 1.39E-02 & $0.00 \mathrm{E}+00$ & $0.00 \mathrm{E}+00$ & $0.00 \mathrm{E}+00$ & $0.00 \mathrm{E}+00$ & $0.00 \mathrm{E}+00$ & $0.00 \mathrm{E}+00$ & $0.00 \mathrm{E}+00$ & $0.00 \mathrm{E}+00$ & $0.00 \mathrm{E}+0 \mathrm{C}$ \\
\hline B 12 & $9.56 \mathrm{E}-03$ & $0.00 \mathrm{E}+00$ & $0.00 \mathrm{E}+00$ & $0.00 \mathrm{E}+00$ & $0.00 \mathrm{E}+00$ & $0.00 \mathrm{E}+00$ & $0.00 \mathrm{E}+00$ & $0.00 E+00$ & $0.00 \mathrm{E}+00$ & $0.00 \mathrm{E}+0 \mathrm{C}$ \\
\hline C 14 & $1.24 \mathrm{E}-02$ & $1.24 \mathrm{E}-02$ & $1.24 \mathrm{E}-02$ & $1.24 \mathrm{E}-02$ & $1.24 \mathrm{E}-02$ & $1.24 \mathrm{E}-02$ & $1.24 \mathrm{E}-02$ & $1.24 \mathrm{E}-02$ & $1.24 \mathrm{E}-02$ & $1.24 \mathrm{E}-02$ \\
\hline C 15 & 8.47E-03 & $0.00 \mathrm{E}+00$ & $0.00 \mathrm{E}+00$ & $0.00 \mathrm{E}+00$ & $0.00 \mathrm{E}+00$ & $0.00 \mathrm{E}+00$ & $0.00 \mathrm{E}+00$ & $0.00 \mathrm{E}+00$ & $0.00 \mathrm{E}+00$ & $0.00 \mathrm{E}+0 \mathrm{C}$ \\
\hline N 16 & $4.99 \mathrm{E}-02$ & $0.00 \mathrm{E}+00$ & $0.00 \mathrm{E}+00$ & $0.00 \mathrm{E}+00$ & $0.00 \mathrm{E}+00$ & $0.00 \mathrm{E}+00$ & $0.00 \mathrm{E}+00$ & $0.00 \mathrm{E}+00$ & $0.00 \mathrm{E}+00$ & $0.00 \mathrm{E}+0 \mathrm{C}$ \\
\hline 019 & $6.06 \mathrm{E}-04$ & $1.25 \mathrm{E}-22$ & $0.00 \mathrm{E}+00$ & $0.00 \mathrm{E}+00$ & $0.00 \mathrm{E}+00$ & $0.00 \mathrm{E}+00$ & $0.00 \mathrm{E}+00$ & $0.00 \mathrm{E}+00$ & $0.00 \mathrm{E}+00$ & $0.00 \mathrm{E}+0 \mathrm{C}$ \\
\hline NE 23 & $1.18 \mathrm{E}+00$ & $3.33 \mathrm{E}-15$ & $0.00 \mathrm{E}+00$ & $0.00 \mathrm{E}+00$ & $0.00 \mathrm{E}+00$ & $0.00 \mathrm{E}+00$ & $0.00 \mathrm{E}+00$ & $0.00 \mathrm{E}+00$ & $0.00 \mathrm{E}+00$ & $0.00 \mathrm{E}+0 \mathrm{C}$ \\
\hline NA 24 & $1.71 \mathrm{E}+02$ & $1.67 \mathrm{E}+02$ & $5.64 \mathrm{E}+01$ & $7.30 \mathrm{E}-02$ & $6.10 \mathrm{E}-13$ & $2.17 \mathrm{E}-27$ & $0.00 \mathrm{E}+00$ & $0.00 \mathrm{E}+00$ & $0.00 \mathrm{E}+00$ & $0.00 \mathrm{E}+0 \mathrm{C}$ \\
\hline NA 24M & $4.52 \mathrm{E}-04$ & $0.00 \mathrm{E}+00$ & $0.00 \mathrm{E}+00$ & $0.00 \mathrm{E}+00$ & $0.00 \mathrm{E}+00$ & $0.00 \mathrm{E}+00$ & $0.00 \mathrm{E}+00$ & $0.00 \mathrm{E}+00$ & $0.00 \mathrm{E}+00$ & $0.00 \mathrm{E}+0 \mathrm{C}$ \\
\hline NA 25 & 7.14E-01 & $5.79 \mathrm{E}-10$ & $0.00 E+00$ & $0.00 \mathrm{E}+00$ & $0.00 E+00$ & $0.00 E+00$ & $0.00 \mathrm{E}+00$ & $0.00 E+00$ & $0.00 \mathrm{E}+00$ & $0.00 \mathrm{E}+0 \mathrm{C}$ \\
\hline MG 27 & $8.04 E+02$ & $8.93 \mathrm{E}+01$ & $0.00 E+00$ & $0.00 E+00$ & $0.00 E+00$ & $0.00 E+00$ & $0.00 \mathrm{E}+00$ & $0.00 E+00$ & $0.00 E+00$ & $0.00 \mathrm{E}+0 \mathrm{C}$ \\
\hline AL 28 & $1.92 \mathrm{E}+04$ & $1.78 \mathrm{E}+00$ & $5.78 \mathrm{E}-07$ & $4.89 \mathrm{E}-09$ & $5.53 \mathrm{E}-17$ & $0.00 \mathrm{E}+00$ & $0.00 \mathrm{E}+00$ & $0.00 \mathrm{E}+00$ & $0.00 \mathrm{E}+00$ & $0.00 \mathrm{E}+0 \mathrm{C}$ \\
\hline AL 29 & $4.18 \mathrm{E}-01$ & $1.72 \mathrm{E}-02$ & $0.00 \mathrm{E}+00$ & $0.00 \mathrm{E}+00$ & $0.00 \mathrm{E}+00$ & $0.00 \mathrm{E}+00$ & $0.00 \mathrm{E}+00$ & $0.00 \mathrm{E}+00$ & $0.00 E+00$ & $0.00 \mathrm{E}+0 \mathrm{C}$ \\
\hline AL 30 & $4.04 \mathrm{E}-04$ & $0.00 E+00$ & $0.00 \mathrm{E}+00$ & $0.00 \mathrm{E}+00$ & $0.00 E+00$ & $0.00 \mathrm{E}+00$ & $0.00 E+00$ & $0.00 \mathrm{E}+00$ & $0.00 \mathrm{E}+00$ & $0.00 \mathrm{E}+0 \mathrm{C}$ \\
\hline SI 31 & $5.78 \mathrm{E}+00$ & $5.06 \mathrm{E}+00$ & $1.02 \mathrm{E}-02$ & 2.97E-19 & $0.00 \mathrm{E}+00$ & $0.00 \mathrm{E}+00$ & $0.00 \mathrm{E}+00$ & $0.00 \mathrm{E}+00$ & $0.00 \mathrm{E}+00$ & $0.00 \mathrm{E}+0 \mathrm{C}$ \\
\hline P 32 & $6.00 \mathrm{E}+00$ & $5.99 \mathrm{E}+00$ & $5.71 \mathrm{E}+00$ & $4.27 \mathrm{E}+00$ & $1.40 \mathrm{E}+00$ & $3.27 \mathrm{E}-01$ & $7.64 \mathrm{E}-02$ & $1.79 \mathrm{E}-02$ & $3.70 \mathrm{E}-04$ & $1.60 \mathrm{E}-07$ \\
\hline P 33 & $2.06 \mathrm{E}-04$ & $2.06 \mathrm{E}-04$ & $2.01 \mathrm{E}-04$ & $1.70 \mathrm{E}-04$ & $8.99 \mathrm{E}-05$ & $3.91 \mathrm{E}-05$ & $1.70 \mathrm{E}-05$ & $7.41 \mathrm{E}-06$ & $8.06 \mathrm{E}-07$ & $9.55 \mathrm{E}-09$ \\
\hline P 34 & $1.16 \mathrm{E}-03$ & $0.00 \mathrm{E}+00$ & $0.00 \mathrm{E}+00$ & $0.00 \mathrm{E}+00$ & $0.00 \mathrm{E}+00$ & $0.00 \mathrm{E}+00$ & $0.00 \mathrm{E}+00$ & $0.00 \mathrm{E}+00$ & $0.00 \mathrm{E}+00$ & $0.00 \mathrm{E}+0 \mathrm{C}$ \\
\hline S 35 & 1.09E-01 & $1.09 \mathrm{E}-01$ & $1.08 \mathrm{E}-01$ & $1.03 \mathrm{E}-01$ & $8.61 \mathrm{E}-02$ & $6.80 \mathrm{E}-02$ & 5.37E-02 & $4.24 \mathrm{E}-02$ & $2.26 \mathrm{E}-02$ & $6.40 \mathrm{E}-03$ \\
\hline S 37 & $3.35 \mathrm{E}-04$ & 5.49E-06 & $0.00 \mathrm{E}+00$ & $0.00 E+00$ & $0.00 E+00$ & $0.00 \mathrm{E}+00$ & $0.00 E+00$ & $0.00 \mathrm{E}+00$ & $0.00 E+00$ & $0.00 \mathrm{E}+0 \mathrm{C}$ \\
\hline CA 45 & 3.77E-03 & 3.77E-03 & $3.75 \mathrm{E}-03$ & $3.66 \mathrm{E}-03$ & 3.31E-03 & $2.92 \mathrm{E}-03$ & $2.57 \mathrm{E}-03$ & $2.26 \mathrm{E}-03$ & $1.61 \mathrm{E}-03$ & $8.14 \mathrm{E}-04$ \\
\hline CA 47 & $3.70 \mathrm{E}-05$ & $3.69 \mathrm{E}-05$ & $3.17 \mathrm{E}-05$ & $1.27 \mathrm{E}-05$ & $3.78 \mathrm{E}-07$ & $3.85 \mathrm{E}-09$ & $3.94 \mathrm{E}-11$ & $4.02 \mathrm{E}-13$ & $1.97 \mathrm{E}-18$ & $4.74 \mathrm{E}-29$ \\
\hline SC 46 & $6.58 \mathrm{E}-01$ & $6.58 \mathrm{E}-01$ & $6.53 \mathrm{E}-01$ & $6.21 \mathrm{E}-01$ & $5.14 \mathrm{E}-01$ & $4.01 \mathrm{E}-01$ & $3.13 \mathrm{E}-01$ & $2.44 \mathrm{E}-01$ & $1.26 \mathrm{E}-01$ & $3.35 \mathrm{E}-02$ \\
\hline
\end{tabular}


TEM-10200-1 $12 / 19 / 17$

Rev.08

Title: AS-RUN NEUTRONICS EVALUATION FOR THE GE HITACHI EXPERIMENT IN THE ATR ECAR NO.: 4740

\begin{tabular}{|c|c|c|c|c|c|c|c|c|c|c|}
\hline \multirow{2}{*}{ Isotope } & EOI & 30.0MI & 1.0D & 7.0D & 30.0D & 60.0D & 90.0D & 120.0D & 200.0D & 360.0D \\
\hline & $\mathrm{Ci}$ & $\mathrm{Ci}$ & $\mathrm{Ci}$ & $\mathrm{Ci}$ & $\mathrm{Ci}$ & $\mathrm{Ci}$ & $\mathrm{Ci}$ & $\mathrm{Ci}$ & $\mathrm{Ci}$ & $\mathrm{Ci}$ \\
\hline SC $46 \mathrm{M}$ & $1.88 \mathrm{E}-05$ & $0.00 \mathrm{E}+00$ & $0.00 \mathrm{E}+00$ & $0.00 E+00$ & $0.00 \mathrm{E}+00$ & $0.00 E+00$ & $0.00 \mathrm{E}+00$ & $0.00 \mathrm{E}+00$ & $0.00 \mathrm{E}+00$ & $0.00 E+00$ \\
\hline SC 47 & $1.22 \mathrm{E}+00$ & $1.22 \mathrm{E}+00$ & $9.93 \mathrm{E}-01$ & 2.87E-01 & $2.47 \mathrm{E}-03$ & $4.98 \mathrm{E}-06$ & $1.02 \mathrm{E}-08$ & $2.17 \mathrm{E}-11$ & $8.85 \mathrm{E}-18$ & $0.00 \mathrm{E}+00$ \\
\hline SC 48 & $1.14 \mathrm{E}-01$ & $1.14 \mathrm{E}-01$ & $7.83 \mathrm{E}-02$ & $8.02 \mathrm{E}-03$ & $1.29 \mathrm{E}-06$ & $1.46 \mathrm{E}-11$ & $1.64 \mathrm{E}-16$ & $1.85 \mathrm{E}-21$ & $0.00 \mathrm{E}+00$ & $0.00 \mathrm{E}+00$ \\
\hline SC 49 & $1.01 \mathrm{E}-01$ & 7.07E-02 & $2.93 \mathrm{E}-09$ & $0.00 \mathrm{E}+00$ & $0.00 \mathrm{E}+00$ & $0.00 \mathrm{E}+00$ & $0.00 \mathrm{E}+00$ & $0.00 \mathrm{E}+00$ & $0.00 \mathrm{E}+00$ & $0.00 \mathrm{E}+00$ \\
\hline SC 50 & $1.94 \mathrm{E}-03$ & $1.00 \mathrm{E}-08$ & $0.00 \mathrm{E}+00$ & $0.00 \mathrm{E}+00$ & $0.00 \mathrm{E}+00$ & $0.00 \mathrm{E}+00$ & $0.00 \mathrm{E}+00$ & $0.00 \mathrm{E}+00$ & $0.00 \mathrm{E}+00$ & $0.00 \mathrm{E}+00$ \\
\hline TI 51 & $2.23 \mathrm{E}+00$ & $6.02 \mathrm{E}-02$ & $0.00 \mathrm{E}+00$ & $0.00 \mathrm{E}+00$ & $0.00 \mathrm{E}+00$ & $0.00 \mathrm{E}+00$ & $0.00 \mathrm{E}+00$ & $0.00 \mathrm{E}+00$ & $0.00 \mathrm{E}+00$ & $0.00 \mathrm{E}+00$ \\
\hline V 52 & $4.05 E+01$ & $1.58 \mathrm{E}-01$ & $0.00 \mathrm{E}+00$ & $0.00 \mathrm{E}+00$ & $0.00 \mathrm{E}+00$ & $0.00 \mathrm{E}+00$ & $0.00 \mathrm{E}+00$ & $0.00 \mathrm{E}+00$ & $0.00 \mathrm{E}+00$ & $0.00 \mathrm{E}+00$ \\
\hline V 53 & $1.86 \mathrm{E}-01$ & 4.57E-07 & $0.00 \mathrm{E}+00$ & $0.00 \mathrm{E}+00$ & $0.00 \mathrm{E}+00$ & $0.00 \mathrm{E}+00$ & $0.00 \mathrm{E}+00$ & $0.00 \mathrm{E}+00$ & $0.00 \mathrm{E}+00$ & $0.00 \mathrm{E}+00$ \\
\hline V 54 & $1.96 \mathrm{E}-03$ & $2.76 \mathrm{E}-13$ & $0.00 \mathrm{E}+00$ & $0.00 \mathrm{E}+00$ & $0.00 \mathrm{E}+00$ & $0.00 \mathrm{E}+00$ & $0.00 \mathrm{E}+00$ & $0.00 \mathrm{E}+00$ & $0.00 \mathrm{E}+00$ & $0.00 \mathrm{E}+00$ \\
\hline CR 51 & $4.01 \mathrm{E}+03$ & $4.01 E+03$ & $3.91 \mathrm{E}+03$ & $3.37 E+03$ & $1.89 \mathrm{E}+03$ & $8.94 \mathrm{E}+02$ & $4.22 \mathrm{E}+02$ & $1.99 \mathrm{E}+02$ & $2.69 \mathrm{E}+01$ & 4.92E-01 \\
\hline CR 55 & $5.58 \mathrm{E}+01$ & $1.60 \mathrm{E}-01$ & $0.00 \mathrm{E}+00$ & $0.00 \mathrm{E}+00$ & $0.00 \mathrm{E}+00$ & $0.00 \mathrm{E}+00$ & $0.00 \mathrm{E}+00$ & $0.00 \mathrm{E}+00$ & $0.00 \mathrm{E}+00$ & $0.00 \mathrm{E}+00$ \\
\hline MN 54 & $7.14 \mathrm{E}+01$ & $7.14 \mathrm{E}+01$ & $7.13 E+01$ & $7.03 E+01$ & $6.68 \mathrm{E}+01$ & $6.25 \mathrm{E}+01$ & $5.85 \mathrm{E}+01$ & $5.47 \mathrm{E}+01$ & $4.58 \mathrm{E}+01$ & $3.21 \mathrm{E}+01$ \\
\hline MN 56 & $8.89 \mathrm{E}+03$ & $7.77 \mathrm{E}+03$ & $1.40 \mathrm{E}+01$ & $2.17 \mathrm{E}-16$ & $0.00 \mathrm{E}+00$ & $0.00 \mathrm{E}+00$ & $0.00 \mathrm{E}+00$ & $0.00 \mathrm{E}+00$ & $0.00 \mathrm{E}+00$ & $0.00 \mathrm{E}+00$ \\
\hline MN 57 & $4.66 \mathrm{E}-01$ & $1.15 \mathrm{E}-06$ & $0.00 \mathrm{E}+00$ & $0.00 \mathrm{E}+00$ & $0.00 \mathrm{E}+00$ & $0.00 \mathrm{E}+00$ & $0.00 \mathrm{E}+00$ & $0.00 \mathrm{E}+00$ & $0.00 \mathrm{E}+00$ & $0.00 \mathrm{E}+00$ \\
\hline MN 58 & $1.44 \mathrm{E}-03$ & $7.26 \mathrm{E}-12$ & $0.00 \mathrm{E}+00$ & $0.00 \mathrm{E}+00$ & $0.00 \mathrm{E}+00$ & $0.00 \mathrm{E}+00$ & $0.00 \mathrm{E}+00$ & $0.00 \mathrm{E}+00$ & $0.00 \mathrm{E}+00$ & $0.00 \mathrm{E}+00$ \\
\hline FE 55 & $2.97 \mathrm{E}+02$ & $2.97 \mathrm{E}+02$ & $2.97 \mathrm{E}+02$ & $2.96 \mathrm{E}+02$ & $2.91 \mathrm{E}+02$ & $2.84 \mathrm{E}+02$ & $2.78 \mathrm{E}+02$ & $2.72 \mathrm{E}+02$ & $2.57 \mathrm{E}+02$ & $2.28 \mathrm{E}+02$ \\
\hline FE 59 & $5.53 \mathrm{E}+01$ & $5.53 E+01$ & $5.44 \mathrm{E}+01$ & $4.96 \mathrm{E}+01$ & $3.48 \mathrm{E}+01$ & $2.19 \mathrm{E}+01$ & $1.38 \mathrm{E}+01$ & $8.71 \mathrm{E}+00$ & $2.54 \mathrm{E}+00$ & $2.16 \mathrm{E}-01$ \\
\hline CO 58 & $3.90 \mathrm{E}+02$ & $3.90 \mathrm{E}+02$ & $3.86 \mathrm{E}+02$ & $3.64 \mathrm{E}+02$ & $2.91 \mathrm{E}+02$ & $2.17 \mathrm{E}+02$ & $1.61 \mathrm{E}+02$ & $1.20 \mathrm{E}+02$ & $5.50 \mathrm{E}+01$ & $1.15 \mathrm{E}+01$ \\
\hline CO 60 & $2.42 \mathrm{E}+02$ & $2.42 \mathrm{E}+02$ & $2.42 \mathrm{E}+02$ & $2.42 \mathrm{E}+02$ & $2.40 \mathrm{E}+02$ & $2.37 \mathrm{E}+02$ & $2.34 \mathrm{E}+02$ & $2.32 \mathrm{E}+02$ & $2.25 \mathrm{E}+02$ & $2.13 \mathrm{E}+02$ \\
\hline $\mathrm{CO} 60 \mathrm{M}$ & $1.69 \mathrm{E}+03$ & $2.32 \mathrm{E}+02$ & $0.00 \mathrm{E}+00$ & $0.00 \mathrm{E}+00$ & $0.00 \mathrm{E}+00$ & $0.00 \mathrm{E}+00$ & $0.00 \mathrm{E}+00$ & $0.00 \mathrm{E}+00$ & $0.00 \mathrm{E}+00$ & $0.00 \mathrm{E}+00$ \\
\hline CO 61 & $8.65 \mathrm{E}+00$ & $7.01 \mathrm{E}+00$ & $3.62 \mathrm{E}-04$ & $0.00 \mathrm{E}+00$ & $0.00 \mathrm{E}+00$ & $0.00 \mathrm{E}+00$ & $0.00 \mathrm{E}+00$ & $0.00 \mathrm{E}+00$ & $0.00 \mathrm{E}+00$ & $0.00 \mathrm{E}+00$ \\
\hline CO 62 & $9.70 \mathrm{E}-02$ & $9.25 \mathrm{E}-08$ & $0.00 \mathrm{E}+00$ & $0.00 \mathrm{E}+00$ & $0.00 \mathrm{E}+00$ & $0.00 \mathrm{E}+00$ & $0.00 \mathrm{E}+00$ & $0.00 \mathrm{E}+00$ & $0.00 \mathrm{E}+00$ & $0.00 \mathrm{E}+00$ \\
\hline NI 59 & $1.12 \mathrm{E}-01$ & $1.12 \mathrm{E}-01$ & $1.12 \mathrm{E}-01$ & $1.12 \mathrm{E}-01$ & $1.12 \mathrm{E}-01$ & $1.12 \mathrm{E}-01$ & $1.12 \mathrm{E}-01$ & $1.12 \mathrm{E}-01$ & $1.12 \mathrm{E}-01$ & $1.12 \mathrm{E}-01$ \\
\hline NI 63 & $1.44 \mathrm{E}+01$ & $1.44 \mathrm{E}+01$ & $1.44 \mathrm{E}+01$ & $1.44 \mathrm{E}+01$ & $1.44 \mathrm{E}+01$ & $1.44 \mathrm{E}+01$ & $1.44 \mathrm{E}+01$ & $1.44 \mathrm{E}+01$ & $1.44 \mathrm{E}+01$ & $1.43 \mathrm{E}+01$ \\
\hline NI 65 & $9.27 \mathrm{E}+01$ & $8.07 \mathrm{E}+01$ & $1.26 \mathrm{E}-01$ & 7.90E-19 & $0.00 \mathrm{E}+00$ & $0.00 \mathrm{E}+00$ & $0.00 \mathrm{E}+00$ & $0.00 \mathrm{E}+00$ & $0.00 \mathrm{E}+00$ & $0.00 \mathrm{E}+00$ \\
\hline NI 66 & $1.41 \mathrm{E}-03$ & $1.40 \mathrm{E}-03$ & $1.04 \mathrm{E}-03$ & $1.68 \mathrm{E}-04$ & $1.52 \mathrm{E}-07$ & $1.63 \mathrm{E}-11$ & $1.75 \mathrm{E}-15$ & $1.88 \mathrm{E}-19$ & $4.91 \mathrm{E}-30$ & $0.00 \mathrm{E}+00$ \\
\hline CU 64 & $5.28 \mathrm{E}+02$ & $5.13 E+02$ & $1.42 \mathrm{E}+02$ & $5.50 \mathrm{E}-02$ & $4.53 \mathrm{E}-15$ & $0.00 \mathrm{E}+00$ & $0.00 \mathrm{E}+00$ & $0.00 \mathrm{E}+00$ & $0.00 \mathrm{E}+00$ & $0.00 \mathrm{E}+00$ \\
\hline CU 66 & $1.14 \mathrm{E}+02$ & $1.94 \mathrm{E}+00$ & $1.04 \mathrm{E}-03$ & $1.68 \mathrm{E}-04$ & $1.52 \mathrm{E}-07$ & $1.63 \mathrm{E}-11$ & $1.76 \mathrm{E}-15$ & $1.89 \mathrm{E}-19$ & $0.00 \mathrm{E}+00$ & $0.00 \mathrm{E}+00$ \\
\hline
\end{tabular}

Page $\mathbf{2 6}$ of $\mathbf{5 7}$
ENGINEERING CALCULATIONS AND ANALYSIS REV. NO.: 0 PROJECT NO.: 32535 Date: 12/02/2019 
TEM-10200-1 $12 / 19 / 17$

Rev.08

Title: AS-RUN NEUTRONICS EVALUATION FOR THE GE HITACHI EXPERIMENT IN THE ATR ECAR NO.: 4740

\begin{tabular}{|c|c|c|c|c|c|c|c|c|c|c|}
\hline \multirow{2}{*}{ Isotope } & EOI & 30.0MI & $1.0 \mathrm{D}$ & 7.0D & 30.0D & 60.0D & 90.0D & 120.0D & 200.0D & 360.0D \\
\hline & $\mathrm{Ci}$ & $\mathbf{C i}$ & $\mathrm{Ci}$ & $\mathbf{C i}$ & $\mathrm{Ci}$ & $\mathrm{Ci}$ & $\mathbf{C i}$ & $\mathbf{C i}$ & $\mathbf{C i}$ & $\mathbf{C i}$ \\
\hline CU 67 & $3.31 \mathrm{E}-04$ & $3.29 \mathrm{E}-04$ & $2.53 \mathrm{E}-04$ & 5.03E-05 & $1.04 \mathrm{E}-07$ & $3.26 \mathrm{E}-11$ & $1.02 \mathrm{E}-14$ & $3.20 \mathrm{E}-18$ & $1.45 \mathrm{E}-27$ & $0.00 \mathrm{E}+00$ \\
\hline ZN 65 & $1.99 \mathrm{E}+01$ & $1.99 \mathrm{E}+01$ & $1.98 \mathrm{E}+01$ & $1.95 \mathrm{E}+01$ & $1.83 \mathrm{E}+01$ & $1.68 \mathrm{E}+01$ & $1.54 \mathrm{E}+01$ & $1.42 \mathrm{E}+01$ & $1.13 \mathrm{E}+01$ & $7.15 E+00$ \\
\hline ZN 69 & $2.85 \mathrm{E}+01$ & $2.04 \mathrm{E}+01$ & 6.14E-01 & $4.35 \mathrm{E}-04$ & $3.65 \mathrm{E}-16$ & $0.00 E+00$ & $0.00 \mathrm{E}+00$ & $0.00 \mathrm{E}+00$ & $0.00 \mathrm{E}+00$ & $0.00 \mathrm{E}+00$ \\
\hline ZN 69M & $1.92 \mathrm{E}+00$ & $1.87 \mathrm{E}+00$ & $5.72 \mathrm{E}-01$ & $4.05 \mathrm{E}-04$ & $3.40 \mathrm{E}-16$ & $0.00 E+00$ & $0.00 \mathrm{E}+00$ & $0.00 \mathrm{E}+00$ & $0.00 \mathrm{E}+00$ & $0.00 \mathrm{E}+00$ \\
\hline ZN 71 & $4.27 \mathrm{E}-02$ & $9.45 \mathrm{E}-06$ & $3.25 \mathrm{E}-08$ & $2.84 \mathrm{E}-19$ & $0.00 \mathrm{E}+00$ & $0.00 E+00$ & $0.00 \mathrm{E}+00$ & $0.00 \mathrm{E}+00$ & $0.00 \mathrm{E}+00$ & $0.00 \mathrm{E}+00$ \\
\hline ZN 71M & $4.48 \mathrm{E}-03$ & $4.10 \mathrm{E}-03$ & $6.43 \mathrm{E}-05$ & $5.62 \mathrm{E}-16$ & $0.00 \mathrm{E}+00$ & $0.00 E+00$ & $0.00 \mathrm{E}+00$ & $0.00 \mathrm{E}+00$ & $0.00 \mathrm{E}+00$ & $0.00 E+00$ \\
\hline GA 70 & $1.48 \mathrm{E}-01$ & $5.52 \mathrm{E}-02$ & $4.22 \mathrm{E}-22$ & $0.00 E+00$ & $0.00 \mathrm{E}+00$ & $0.00 E+00$ & $0.00 \mathrm{E}+00$ & $0.00 \mathrm{E}+00$ & $0.00 \mathrm{E}+00$ & $0.00 \mathrm{E}+00$ \\
\hline GA 72 & $5.47 \mathrm{E}-04$ & $5.34 \mathrm{E}-04$ & $1.68 \mathrm{E}-04$ & $1.42 \mathrm{E}-07$ & $2.32 \mathrm{E}-19$ & $0.00 E+00$ & $0.00 \mathrm{E}+00$ & $0.00 \mathrm{E}+00$ & $0.00 \mathrm{E}+00$ & $0.00 E+00$ \\
\hline GA 72M & $1.69 \mathrm{E}-05$ & $0.00 \mathrm{E}+00$ & $0.00 \mathrm{E}+00$ & $0.00 E+00$ & $0.00 \mathrm{E}+00$ & $0.00 E+00$ & $0.00 E+00$ & $0.00 \mathrm{E}+00$ & $0.00 \mathrm{E}+00$ & $0.00 \mathrm{E}+00$ \\
\hline GE 71 & $2.13 \mathrm{E}-04$ & $2.13 \mathrm{E}-04$ & 2.01E-04 & $1.41 \mathrm{E}-04$ & $3.66 \mathrm{E}-05$ & $6.29 \mathrm{E}-06$ & $1.08 \mathrm{E}-06$ & $1.85 \mathrm{E}-07$ & 1.69E-09 & $1.40 \mathrm{E}-13$ \\
\hline GE 71M & $2.03 \mathrm{E}-05$ & $0.00 \mathrm{E}+00$ & $0.00 \mathrm{E}+00$ & $0.00 \mathrm{E}+00$ & $0.00 \mathrm{E}+00$ & $0.00 \mathrm{E}+00$ & $0.00 \mathrm{E}+00$ & $0.00 \mathrm{E}+00$ & $0.00 \mathrm{E}+00$ & $0.00 \mathrm{E}+00$ \\
\hline SR 89 & 1.57E-02 & $1.57 \mathrm{E}-02$ & $1.55 \mathrm{E}-02$ & $1.42 \mathrm{E}-02$ & $1.04 \mathrm{E}-02$ & $6.88 \mathrm{E}-03$ & $4.56 \mathrm{E}-03$ & $3.02 \mathrm{E}-03$ & $1.01 \mathrm{E}-03$ & 1.12E-04 \\
\hline SR 90 & 7.15E-08 & $7.15 \mathrm{E}-08$ & 7.15E-08 & 7.15E-08 & $7.14 \mathrm{E}-08$ & 7.13E-08 & $7.11 \mathrm{E}-08$ & $7.10 \mathrm{E}-08$ & $7.06 \mathrm{E}-08$ & 6.99E-08 \\
\hline SR 91 & $3.17 \mathrm{E}-03$ & $3.06 \mathrm{E}-03$ & 5.50E-04 & $1.51 \mathrm{E}-08$ & $4.85 \mathrm{E}-26$ & $0.00 E+00$ & $0.00 \mathrm{E}+00$ & $0.00 \mathrm{E}+00$ & $0.00 \mathrm{E}+00$ & $0.00 \mathrm{E}+00$ \\
\hline SR 93 & $1.09 \mathrm{E}-05$ & $6.83 \mathrm{E}-07$ & $0.00 \mathrm{E}+00$ & $0.00 E+00$ & $0.00 \mathrm{E}+00$ & $0.00 E+00$ & $0.00 \mathrm{E}+00$ & $0.00 \mathrm{E}+00$ & $0.00 \mathrm{E}+00$ & $0.00 \mathrm{E}+00$ \\
\hline Y 89M & 7.77E-03 & 7.74E-03 & $6.29 E-03$ & $1.76 \mathrm{E}-03$ & $1.34 \mathrm{E}-05$ & $2.32 \mathrm{E}-08$ & $4.01 \mathrm{E}-11$ & $6.95 \mathrm{E}-14$ & $2.98 \mathrm{E}-21$ & $0.00 \mathrm{E}+00$ \\
\hline Y 90 & $3.42 E+01$ & $3.40 \mathrm{E}+01$ & $2.64 \mathrm{E}+01$ & $5.55 \mathrm{E}+00$ & $1.40 \mathrm{E}-02$ & 5.83E-06 & $7.35 \mathrm{E}-08$ & 7.10E-08 & $7.06 \mathrm{E}-08$ & $6.99 \mathrm{E}-08$ \\
\hline Y 90M & $2.52 \mathrm{E}-02$ & $2.26 \mathrm{E}-02$ & $1.18 \mathrm{E}-04$ & $1.23 \mathrm{E}-18$ & $0.00 \mathrm{E}+00$ & $0.00 E+00$ & $0.00 \mathrm{E}+00$ & $0.00 E+00$ & $0.00 \mathrm{E}+00$ & $0.00 \mathrm{E}+00$ \\
\hline Y 91 & $4.13 \mathrm{E}-02$ & $4.13 \mathrm{E}-02$ & $4.08 \mathrm{E}-02$ & $3.80 \mathrm{E}-02$ & $2.89 \mathrm{E}-02$ & 2.03E-02 & $1.42 \mathrm{E}-02$ & 9.96E-03 & $3.86 \mathrm{E}-03$ & 5.80E-04 \\
\hline Y 92 & $3.57 \mathrm{E}-02$ & $3.24 \mathrm{E}-02$ & $3.25 \mathrm{E}-04$ & $1.83 \mathrm{E}-16$ & $0.00 \mathrm{E}+00$ & $0.00 E+00$ & $0.00 \mathrm{E}+00$ & $0.00 \mathrm{E}+00$ & $0.00 \mathrm{E}+00$ & $0.00 \mathrm{E}+00$ \\
\hline Y 93 & $1.09 \mathrm{E}-05$ & $1.07 \mathrm{E}-05$ & $2.13 \mathrm{E}-06$ & $1.09 \mathrm{E}-10$ & $3.84 \mathrm{E}-27$ & $0.00 \mathrm{E}+00$ & $0.00 \mathrm{E}+00$ & $0.00 \mathrm{E}+00$ & $0.00 \mathrm{E}+00$ & $0.00 \mathrm{E}+00$ \\
\hline Y 94 & $2.27 \mathrm{E}-03$ & 7.64E-04 & $0.00 \mathrm{E}+00$ & $0.00 E+00$ & $0.00 \mathrm{E}+00$ & $0.00 E+00$ & $0.00 \mathrm{E}+00$ & $0.00 \mathrm{E}+00$ & $0.00 \mathrm{E}+00$ & $0.00 \mathrm{E}+00$ \\
\hline Y 96 & $1.46 \mathrm{E}-05$ & $1.72 \mathrm{E}-09$ & $0.00 \mathrm{E}+00$ & $0.00 \mathrm{E}+00$ & $0.00 \mathrm{E}+00$ & $0.00 \mathrm{E}+00$ & $0.00 \mathrm{E}+00$ & $0.00 \mathrm{E}+00$ & $0.00 \mathrm{E}+00$ & $0.00 \mathrm{E}+00$ \\
\hline ZR 89 & 7.79E-03 & $7.75 \mathrm{E}-03$ & $6.30 \mathrm{E}-03$ & $1.76 \mathrm{E}-03$ & $1.34 \mathrm{E}-05$ & $2.33 \mathrm{E}-08$ & $4.02 \mathrm{E}-11$ & $6.96 \mathrm{E}-14$ & $2.98 \mathrm{E}-21$ & $0.00 \mathrm{E}+00$ \\
\hline ZR 93 & $1.08 \mathrm{E}-05$ & $1.08 \mathrm{E}-05$ & $1.08 \mathrm{E}-05$ & $1.08 \mathrm{E}-05$ & $1.08 \mathrm{E}-05$ & $1.08 \mathrm{E}-05$ & $1.08 \mathrm{E}-05$ & $1.08 \mathrm{E}-05$ & $1.08 \mathrm{E}-05$ & $1.08 \mathrm{E}-05$ \\
\hline ZR 95 & $1.31 \mathrm{E}+01$ & $1.30 \mathrm{E}+01$ & $1.29 \mathrm{E}+01$ & $1.21 \mathrm{E}+01$ & $9.43 \mathrm{E}+00$ & $6.81 E+00$ & $4.92 \mathrm{E}+00$ & $3.56 \mathrm{E}+00$ & $1.49 \mathrm{E}+00$ & $2.64 \mathrm{E}-01$ \\
\hline ZR 97 & $2.10 \mathrm{E}+01$ & $2.06 \mathrm{E}+01$ & $7.85 E+00$ & $2.14 \mathrm{E}-02$ & $3.14 \mathrm{E}-12$ & $4.70 \mathrm{E}-25$ & $0.00 \mathrm{E}+00$ & $0.00 \mathrm{E}+00$ & $0.00 \mathrm{E}+00$ & $0.00 E+00$ \\
\hline NB 92 & $1.55 \mathrm{E}+00$ & $1.55 \mathrm{E}+00$ & $1.45 \mathrm{E}+00$ & 9.61E-01 & $2.00 \mathrm{E}-01$ & $2.59 \mathrm{E}-02$ & $3.34 \mathrm{E}-03$ & $4.31 \mathrm{E}-04$ & $1.84 \mathrm{E}-06$ & $3.34 \mathrm{E}-11$ \\
\hline
\end{tabular}

Page $\mathbf{2 7}$ of $\mathbf{5 7}$
ENGINEERING CALCULATIONS AND ANALYSIS

\author{
Page 27 of 57
}


TEM-10200-1 $12 / 19 / 17$

Rev.08

Title: AS-RUN NEUTRONICS EVALUATION FOR THE GE HITACHI EXPERIMENT IN THE ATR ECAR NO.: 4740

\begin{tabular}{|c|c|c|c|c|c|c|c|c|c|c|}
\hline \multirow{2}{*}{ Isotope } & EOI & 30.0MI & $1.0 \mathrm{D}$ & 7.0D & 30.0D & 60.0D & 90.0D & 120.0D & 200.0D & 360.0D \\
\hline & $\mathbf{C i}$ & $\mathbf{C i}$ & $\mathrm{Ci}$ & $\mathbf{C i}$ & $\mathrm{Ci}$ & $\mathrm{Ci}$ & $\mathbf{C i}$ & $\mathbf{C i}$ & $\mathbf{C i}$ & $\mathbf{C i}$ \\
\hline NB 93M & $1.56 \mathrm{E}-07$ & $1.56 \mathrm{E}-07$ & $1.58 \mathrm{E}-07$ & 1.66E-07 & $1.98 \mathrm{E}-07$ & $2.41 \mathrm{E}-07$ & $2.83 \mathrm{E}-07$ & $3.24 \mathrm{E}-07$ & $4.35 \mathrm{E}-07$ & $6.53 \mathrm{E}-07$ \\
\hline NB 94 & $1.92 \mathrm{E}-02$ & $1.92 \mathrm{E}-02$ & $1.92 \mathrm{E}-02$ & $1.92 \mathrm{E}-02$ & $1.92 \mathrm{E}-02$ & $1.92 \mathrm{E}-02$ & $1.92 \mathrm{E}-02$ & $1.92 \mathrm{E}-02$ & $1.92 \mathrm{E}-02$ & $1.92 \mathrm{E}-02$ \\
\hline NB 95 & $4.28 \mathrm{E}+01$ & $4.28 \mathrm{E}+01$ & $4.22 \mathrm{E}+01$ & $3.89 E+01$ & $2.86 \mathrm{E}+01$ & $1.94 \mathrm{E}+01$ & $1.33 \mathrm{E}+01$ & $9.19 \mathrm{E}+00$ & $3.59 \mathrm{E}+00$ & 5.97E-01 \\
\hline NB 95M & $9.08 \mathrm{E}-02$ & $9.08 \mathrm{E}-02$ & $9.08 \mathrm{E}-02$ & 8.81E-02 & 6.99E-02 & 5.05E-02 & $3.65 \mathrm{E}-02$ & $2.64 \mathrm{E}-02$ & $1.11 \mathrm{E}-02$ & 1.96E-03 \\
\hline NB 96 & $1.39 \mathrm{E}-01$ & $1.37 \mathrm{E}-01$ & $6.82 \mathrm{E}-02$ & $9.49 \mathrm{E}-04$ & $7.26 \mathrm{E}-11$ & $3.79 \mathrm{E}-20$ & $1.98 \mathrm{E}-29$ & $0.00 \mathrm{E}+00$ & $0.00 \mathrm{E}+00$ & $0.00 \mathrm{E}+00$ \\
\hline NB 97 & $2.10 \mathrm{E}+01$ & $2.09 \mathrm{E}+01$ & $7.89 \mathrm{E}+00$ & $2.15 \mathrm{E}-02$ & $3.39 \mathrm{E}-12$ & $4.80 \mathrm{E}-25$ & $0.00 \mathrm{E}+00$ & $0.00 \mathrm{E}+00$ & $0.00 \mathrm{E}+00$ & $0.00 E+00$ \\
\hline NB 97M & $1.99 \mathrm{E}+01$ & $1.95 \mathrm{E}+01$ & $7.43 E+00$ & $2.02 \mathrm{E}-02$ & $2.98 \mathrm{E}-12$ & $4.45 E-25$ & $0.00 \mathrm{E}+00$ & $0.00 \mathrm{E}+00$ & $0.00 \mathrm{E}+00$ & $0.00 \mathrm{E}+00$ \\
\hline NB 98 & $3.26 \mathrm{E}-03$ & $0.00 E+00$ & $0.00 E+00$ & $0.00 E+00$ & $0.00 \mathrm{E}+00$ & $0.00 E+00$ & $0.00 \mathrm{E}+00$ & $0.00 \mathrm{E}+00$ & $0.00 \mathrm{E}+00$ & $0.00 E+00$ \\
\hline NB100 & $1.62 \mathrm{E}-04$ & $0.00 \mathrm{E}+00$ & $0.00 \mathrm{E}+00$ & $0.00 E+00$ & $0.00 \mathrm{E}+00$ & $0.00 E+00$ & $0.00 E+00$ & $0.00 \mathrm{E}+00$ & $0.00 \mathrm{E}+00$ & $0.00 \mathrm{E}+00$ \\
\hline MO 93M & $4.50 \mathrm{E}-01$ & $4.28 \mathrm{E}-01$ & $3.97 \mathrm{E}-02$ & $1.86 \mathrm{E}-08$ & $0.00 \mathrm{E}+00$ & $0.00 E+00$ & $0.00 \mathrm{E}+00$ & $0.00 \mathrm{E}+00$ & $0.00 \mathrm{E}+00$ & $0.00 \mathrm{E}+00$ \\
\hline MO 93 & $1.65 \mathrm{E}-03$ & $1.65 \mathrm{E}-03$ & $1.65 \mathrm{E}-03$ & 1.65E-03 & $1.65 \mathrm{E}-03$ & 1.65E-03 & $1.65 \mathrm{E}-03$ & $1.65 \mathrm{E}-03$ & $1.65 \mathrm{E}-03$ & 1.65E-03 \\
\hline MO 99 & $3.15 E+02$ & $3.13 E+02$ & $2.45 \mathrm{E}+02$ & $5.40 \mathrm{E}+01$ & $1.64 \mathrm{E}-01$ & 8.52E-05 & $4.43 \mathrm{E}-08$ & $2.31 \mathrm{E}-11$ & $4.03 E-20$ & $0.00 \mathrm{E}+00$ \\
\hline M0101 & $7.83 \mathrm{E}+01$ & $1.89 \mathrm{E}+01$ & $0.00 \mathrm{E}+00$ & $0.00 \mathrm{E}+00$ & $0.00 \mathrm{E}+00$ & $0.00 \mathrm{E}+00$ & $0.00 \mathrm{E}+00$ & $0.00 \mathrm{E}+00$ & $0.00 \mathrm{E}+00$ & $0.00 \mathrm{E}+00$ \\
\hline TC 99 & $7.20 \mathrm{E}-05$ & $7.20 \mathrm{E}-05$ & $7.23 \mathrm{E}-05$ & 7.31E-05 & $7.34 \mathrm{E}-05$ & 7.34E-05 & $7.34 \mathrm{E}-05$ & $7.34 \mathrm{E}-05$ & $7.34 \mathrm{E}-05$ & 7.34E-05 \\
\hline TC100 & $3.37 E+00$ & $0.00 \mathrm{E}+00$ & $0.00 \mathrm{E}+00$ & $0.00 E+00$ & $0.00 \mathrm{E}+00$ & $0.00 E+00$ & $0.00 \mathrm{E}+00$ & $0.00 \mathrm{E}+00$ & $0.00 \mathrm{E}+00$ & $0.00 \mathrm{E}+00$ \\
\hline TC101 & $7.83 \mathrm{E}+01$ & $4.52 \mathrm{E}+01$ & $0.00 \mathrm{E}+00$ & $0.00 \mathrm{E}+00$ & $0.00 \mathrm{E}+00$ & $0.00 \mathrm{E}+00$ & $0.00 \mathrm{E}+00$ & $0.00 \mathrm{E}+00$ & $0.00 \mathrm{E}+00$ & $0.00 \mathrm{E}+00$ \\
\hline RU103 & $1.84 \mathrm{E}-03$ & $1.84 \mathrm{E}-03$ & $1.81 \mathrm{E}-03$ & $1.63 \mathrm{E}-03$ & $1.09 \mathrm{E}-03$ & 6.39E-04 & $3.77 \mathrm{E}-04$ & $2.22 \mathrm{E}-04$ & $5.41 \mathrm{E}-05$ & $3.21 \mathrm{E}-06$ \\
\hline RH104 & 1.91E-05 & 1.37E-08 & $0.00 E+00$ & $0.00 E+00$ & $0.00 \mathrm{E}+00$ & $0.00 E+00$ & $0.00 \mathrm{E}+00$ & $0.00 E+00$ & $0.00 \mathrm{E}+00$ & $0.00 \mathrm{E}+00$ \\
\hline LU176M & 4.07E-01 & 3.70E-01 & $4.48 \mathrm{E}-03$ & $8.02 \mathrm{E}-15$ & $0.00 \mathrm{E}+00$ & $0.00 E+00$ & $0.00 \mathrm{E}+00$ & $0.00 \mathrm{E}+00$ & $0.00 \mathrm{E}+00$ & $0.00 \mathrm{E}+00$ \\
\hline LU177 & $8.02 \mathrm{E}-02$ & $8.00 \mathrm{E}-02$ & $7.23 \mathrm{E}-02$ & 3.89E-02 & $3.63 \mathrm{E}-03$ & $1.76 \mathrm{E}-04$ & $1.85 \mathrm{E}-05$ & $1.01 \mathrm{E}-05$ & $6.82 \mathrm{E}-06$ & $3.33 \mathrm{E}-06$ \\
\hline LU177M & $7.25 \mathrm{E}-05$ & $7.25 \mathrm{E}-05$ & $7.22 \mathrm{E}-05$ & 7.03E-05 & $6.34 \mathrm{E}-05$ & 5.54E-05 & $4.85 \mathrm{E}-05$ & $4.24 \mathrm{E}-05$ & $2.96 \mathrm{E}-05$ & $1.45 \mathrm{E}-05$ \\
\hline HF175 & $3.55 E+00$ & $3.55 E+00$ & $3.51 E+00$ & $3.31 E+00$ & $2.64 \mathrm{E}+00$ & $1.96 \mathrm{E}+00$ & $1.46 \mathrm{E}+00$ & $1.08 \mathrm{E}+00$ & $4.90 \mathrm{E}-01$ & 1.00E-01 \\
\hline HF178M & $1.30 \mathrm{E}+00$ & $0.00 \mathrm{E}+00$ & $0.00 \mathrm{E}+00$ & $0.00 \mathrm{E}+00$ & $0.00 \mathrm{E}+00$ & $0.00 \mathrm{E}+00$ & $0.00 \mathrm{E}+00$ & $0.00 \mathrm{E}+00$ & $0.00 \mathrm{E}+00$ & $0.00 \mathrm{E}+00$ \\
\hline HF179M & $7.19 \mathrm{E}+02$ & $0.00 \mathrm{E}+00$ & $0.00 \mathrm{E}+00$ & $0.00 E+00$ & $0.00 \mathrm{E}+00$ & $0.00 \mathrm{E}+00$ & $0.00 \mathrm{E}+00$ & $0.00 \mathrm{E}+00$ & $0.00 \mathrm{E}+00$ & $0.00 \mathrm{E}+00$ \\
\hline HF180M & $2.85 \mathrm{E}+00$ & $2.68 \mathrm{E}+00$ & $1.38 \mathrm{E}-01$ & $1.82 \mathrm{E}-09$ & $0.00 \mathrm{E}+00$ & $0.00 E+00$ & $0.00 \mathrm{E}+00$ & $0.00 \mathrm{E}+00$ & $0.00 \mathrm{E}+00$ & $0.00 \mathrm{E}+00$ \\
\hline HF181 & $5.75 \mathrm{E}+01$ & $5.75 \mathrm{E}+01$ & $5.66 \mathrm{E}+01$ & $5.13 E+01$ & $3.52 \mathrm{E}+01$ & $2.16 \mathrm{E}+01$ & $1.32 \mathrm{E}+01$ & $8.09 E+00$ & $2.19 \mathrm{E}+00$ & 1.60E-01 \\
\hline TA182 & $2.95 \mathrm{E}+01$ & $2.95 \mathrm{E}+01$ & $2.93 E+01$ & $2.83 E+01$ & $2.46 \mathrm{E}+01$ & $2.06 \mathrm{E}+01$ & $1.72 \mathrm{E}+01$ & $1.43 \mathrm{E}+01$ & $8.84 \mathrm{E}+00$ & $3.37 E+00$ \\
\hline TA182M & $8.84 \mathrm{E}-02$ & $2.51 \mathrm{E}-02$ & $0.00 E+00$ & $0.00 E+00$ & $0.00 \mathrm{E}+00$ & $0.00 E+00$ & $0.00 \mathrm{E}+00$ & $0.00 \mathrm{E}+00$ & $0.00 \mathrm{E}+00$ & $0.00 E+00$ \\
\hline
\end{tabular}

Page 28 of $\mathbf{5 7}$
ENGINEERING CALCULATIONS AND ANALYSIS PROJECT NO.: 32535 Date: 12/02/2019 
TEM-10200-1

$12 / 19 / 17$

Rev.08

Title: AS-RUN NEUTRONICS EVALUATION FOR THE GE HITACHI EXPERIMENT IN THE ATR ECAR NO.: 4740

\section{ENGINEERING CALCULATIONS AND ANALYSIS}

Page $\mathbf{2 9}$ of $\mathbf{5 7}$

\begin{tabular}{|c|c|c|c|c|c|c|c|c|c|c|}
\hline \multirow{2}{*}{ Isotope } & EOI & 30.0MI & 1.0D & 7.0D & 30.0D & 60.0D & 90.0D & 120.0D & 200.0D & 360.0D \\
\hline & $\mathrm{Ci}$ & $\mathrm{Ci}$ & $\mathbf{C i}$ & $\mathrm{Ci}$ & $\mathrm{Ci}$ & $\mathrm{Ci}$ & $\mathrm{Ci}$ & $\mathbf{C i}$ & Ci & $\mathrm{Ci}$ \\
\hline TA183 & $1.55 \mathrm{E}+02$ & $1.54 \mathrm{E}+02$ & $1.35 \mathrm{E}+02$ & $5.97 \mathrm{E}+01$ & $2.62 \mathrm{E}+00$ & $4.44 \mathrm{E}-02$ & $7.53 \mathrm{E}-04$ & $1.28 \mathrm{E}-05$ & $2.42 \mathrm{E}-10$ & $8.71 \mathrm{E}-20$ \\
\hline W183M & $1.17 \mathrm{E}-02$ & $0.00 \mathrm{E}+00$ & $0.00 \mathrm{E}+00$ & $0.00 \mathrm{E}+00$ & $0.00 \mathrm{E}+00$ & $0.00 \mathrm{E}+00$ & $0.00 \mathrm{E}+00$ & $0.00 \mathrm{E}+00$ & $0.00 \mathrm{E}+00$ & $0.00 \mathrm{E}+00$ \\
\hline W185 & $1.11 \mathrm{E}-02$ & $1.11 \mathrm{E}-02$ & $1.10 \mathrm{E}-02$ & $1.04 \mathrm{E}-02$ & 8.41E-03 & $6.38 \mathrm{E}-03$ & $4.84 \mathrm{E}-03$ & $3.67 \mathrm{E}-03$ & $1.75 \mathrm{E}-03$ & $4.00 \mathrm{E}-04$ \\
\hline W185M & $3.02 \mathrm{E}-05$ & $1.18 \mathrm{E}-10$ & $0.00 \mathrm{E}+00$ & $0.00 \mathrm{E}+00$ & $0.00 \mathrm{E}+00$ & $0.00 \mathrm{E}+00$ & $0.00 \mathrm{E}+00$ & $0.00 \mathrm{E}+00$ & $0.00 \mathrm{E}+00$ & $0.00 \mathrm{E}+00$ \\
\hline W187 & $2.00 \mathrm{E}-06$ & $1.98 \mathrm{E}-06$ & 9.99E-07 & $1.53 \mathrm{E}-08$ & $1.71 \mathrm{E}-15$ & $1.46 \mathrm{E}-24$ & $0.00 \mathrm{E}+00$ & $0.00 \mathrm{E}+00$ & $0.00 \mathrm{E}+00$ & $0.00 \mathrm{E}+00$ \\
\hline RE186 & $1.14 \mathrm{E}-03$ & $1.13 \mathrm{E}-03$ & 9.47E-04 & $3.15 \mathrm{E}-04$ & 4.62E-06 & $1.88 \mathrm{E}-08$ & $7.63 \mathrm{E}-11$ & $3.10 \mathrm{E}-13$ & $1.30 \mathrm{E}-19$ & $0.00 \mathrm{E}+00$ \\
\hline SUMTOT & $3.83 E+04$ & $1.48 \mathrm{E}+04$ & $5.79 \mathrm{E}+03$ & $4.68 \mathrm{E}+03$ & $2.96 \mathrm{E}+03$ & $1.82 \mathrm{E}+03$ & $1.25 \mathrm{E}+03$ & $9.52 \mathrm{E}+02$ & $6.55 \mathrm{E}+02$ & $5.12 \mathrm{E}+02$ \\
\hline OTOTAL & $3.83 E+04$ & $1.48 \mathrm{E}+04$ & $5.79 \mathrm{E}+03$ & $4.68 \mathrm{E}+03$ & $2.96 \mathrm{E}+03$ & $1.82 \mathrm{E}+03$ & $1.25 E+03$ & $9.52 \mathrm{E}+02$ & $6.55 \mathrm{E}+02$ & $5.12 \mathrm{E}+02$ \\
\hline
\end{tabular}


Rev.08

Title: $\quad$ AS-RUN NEUTRONICS EVALUATION FOR THE GE HITACHI EXPERIMENT IN THE ATR

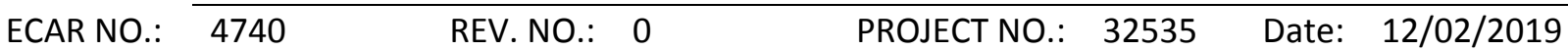

\section{REFERENCES}

[1] N. H. Manwaring Letter to R. R. Little, Advanced Test Reactor Power History Through Cycle 164B-1, January 21, 2019, RE-03-19 (see Appendix B).

[2] Tim Goorley, Jeffrey S. Bull, Forrest B. Brown, et. al., "Release of MCNP5_RSICC_1.30," Trans. Am. Nucl. Soc., Vol. 91, 693-694 (2004).

[3] X-5 Monte Carlo Team, "MCNP - A General Monte Carlo N-Particle Transport Code, Version 5," Volume I, LA-UR-03-1987, Los Alamos National Laboratory, April 24, 2003 (Revised /30/2004) and Volume II, LA-CP-0245, Los Alamos National Laboratory, April 24, 2003 (Revise $6 / 30 / 2004$ ) (Vol. II is available with a licensed copy of MCNP).

[4] A. G. Croff, ORIGEN2: A Versatile Computer code for Calculating the Nuclide Compositions and Characteristics of Nuclear Materials, Nuclear Technology, Vol. 62, pp. 335-352, (1983).

[5] W. M. Cowherd, MCNP Verification and Validation For Use by the INL Neutronic Analysis Group, PLN-5021, Revision 0, January 6, 2016 (EA ID 234728).

[6] B. G. Schnitzler, "Origen2 Cross Section Library Assessment for ATR Applications," BGS-6-91, Idaho National Laboratory, April 1991.

[7] J.R. Mitchell, J.W. Nielsen, "Software Management ORIGEN2 Version 2.2," PLN-3710, Revision 0, October 2011, INL Record Number 3818329, January 25, 2012 (EA ID 201298).

[8] T.A. Tomberlin, "Advanced Test Reactor Critical Facility Measurements in Support of Advanced Test Reactor Experiments," TRA-ATRC-1546, Rev. 0, March 2000.

[9] ECAR-3569, "Physics Analysis of the GE Hitachi Experiment," Rev. 2, B. J. Gross, May 23, 2017.

[10] TEV-2944, "Verification and Validation Testing of MCNP and ORIGEN2 Computer Codes for Idaho National Laboratory (INL) High Performance Computing (HPC) Systems," Rev. 4, J. W. Nielsen, July 16, 2019. 
TEM-10200-1

$12 / 19 / 17$

ENGINEERING CALCULATIONS AND ANALYSIS

Rev.08

Title: $\quad$ AS-RUN NEUTRONICS EVALUATION FOR THE GE HITACHI EXPERIMENT IN THE ATR

ECAR NO.: 4740

REV. NO.: 0

PROJECT NO.: 32535 Date: 12/02/2019

\section{APPENDIX A “PYHSICS ANALYSIS REQUEST FORM”}

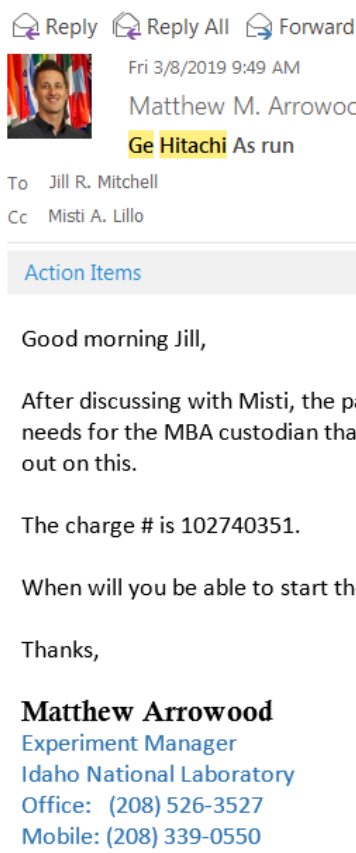


TEM-10200-1

Rev.08

Title: AS-RUN NEUTRONICS EVALUATION FOR THE GE HITACHI EXPERIMENT IN THE ATR

ECAR NO.:

4740

REV. NO.: 0

PROJECT NO.: 32535 Date: 12/02/2019

\section{APPENDIX B “POWER HISTORY LETTER”}

\section{INTEROFFICE MEMORANDUM}

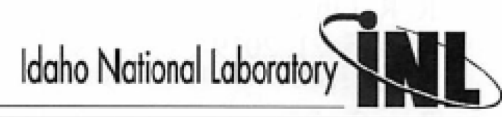

Date:

January 21,2019

To:

R. R. Little, Reactor and Nuclear Safety Engineering Manager, Advanced Test

Reactor Programs

From: Reactor Engineering, Advanced Test Reactor Programs

Subject: Advanced Test Reactor Power History Through Cycle 164B-1

References: (a) A. V. Briscoe letter to J. L. Durney, ATR Power History Through Cycle 34C-1, June 7, 1977, AVB-9-77

(b) C. C. Swanson letter to J. L. Durney, ATR Power History Through Cycle 72A-1, February 3, 1986, CAS-05-86

(c) L. S. Loret letter to E. C. Anderson, Sr., ATR Power History Through Cycle 102B-1, February 28, 1994, LSL-11-94

(d) D. E. Hale letter to J. C. Chapman, ATR Power History Through Cycle 133B-1, August 18, 2004, DEH-05-04

Table 1 lists the Advanced Test Reactor (ATR) N-16 constrained power history data since the Beryllium VI Core Internals Changeout (CIC) Cycle 134A-1 through Cycle 164B-1.

Table 2 lists the accumulated N-16 lobe and total core exposure, as obtained from the ATR Data Acquisition System (RDAS) for Cycles 134A-1 through 164B-1.

The ATR power history prior to Cycle $134 \mathrm{~A}-1$ is presented in the references.

Table 1. Summary of ATR Power History by Cycle

\begin{tabular}{|c|c|c|c|c|c|c|c|c|c|c|c|c|}
\hline & \multicolumn{5}{|c|}{ Average Lobe Power (MW) } & \multicolumn{6}{|c|}{ Cycle Exposure (MWd) } & \multirow{2}{*}{$\begin{array}{l}\text { Length } \\
\text { (EFPD) }\end{array}$} \\
\hline & NW & $\mathrm{NE}$ & $\mathrm{C}$ & SW & SE & NW & NE & C & SW & SE & Total & \\
\hline $134 \mathrm{~A}$ & - & - & - & - & - & - & - & $=$ & - & - & - & - \\
\hline & - & - & - & - & - & .21 & .30 & & & 0.36 & 1.62 & - \\
\hline & 8.0 & 8.0 & 24.5 & 23.0 & E & 452.82 & & & & & 727.59 & 25.10 \\
\hline 134 & 18.0 & 18.0 & 25. & 23.0 & 2 & 52 & 30 & 0 & 4 & 536.07 & & \\
\hline & 0 & 18 & 20 & & & & & & & & 83 & 13.10 \\
\hline & 0 & 18.0 & 24 & & & & & & & & $2,769.20$ & 25.50 \\
\hline & 18.0 & 18.0 & 25 & & 25.0 & 40 & & & 51 & 562.99 & $2,458.91$ & \\
\hline & 18.0 & 18.0 & 25 & 23 & & & & & 93 & 86 & $4,419.92$ & 60 \\
\hline & 18.0 & 18.0 & 24 & & 0 & & & & & 0.17 & $5,391.12$ & 50.90 \\
\hline & & & & & & & 32 & & & 896.97 & $4,129.35$ & 39.00 \\
\hline & 18.0 & 18.0 & 24.7 & 20.0 & 25.0 & 97 & & & & & & \\
\hline 13 & 20.0 & 17.9 & 35.5 & 56.6 & 30 & 241 & 217.01 & & & 36 & $1,941.61$ & 12.10 \\
\hline & 18.0 & 18.0 & 23.6 & 23.0 & 25.0 & $1,046.87$ & $1,047.69$ & 1,3 & 1,336 & $1,453.47$ & $6,255.11$ & 58.10 \\
\hline & 18.0 & 18.0 & 23. & 23.0 & & & & & & & & 46.60 \\
\hline $139 \mathrm{~A}-1$ & 18.0 & 18.0 & 23.9 & 3.0 & 25.0 & 928.73 & 929.09 & $1,231.07$ & $1,186.27$ & $1,289.47$ & $5,564.63$ & 51.60 \\
\hline
\end{tabular}


Rev.08 ECAR NO AS-RUN NEUTRONICS EVALUATION FOR THE GE HITACHI EXPERIMENT IN THE ATR

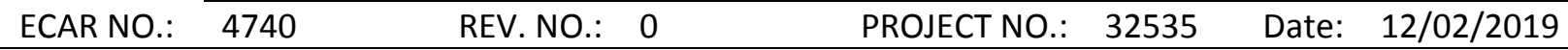

R. R. Little

January 21,2019

RE-03-19

Page 2 of 5

Table 1. Summary of ATR Power History by Cycle

\begin{tabular}{|c|c|c|c|c|c|c|c|c|c|c|c|c|}
\hline & \multicolumn{5}{|c|}{ Average Lobe Power (MV } & \multicolumn{6}{|c|}{ Cycle Exposure (MWd) } & \multirow{2}{*}{$\begin{array}{l}\text { Length } \\
\text { (EFPD) }\end{array}$} \\
\hline & W & NE & $\mathrm{C}$ & SW & SE & NW & NE & $\mathrm{C}$ & SW & SE & INow & \\
\hline & & 18.0 & 23.2 & 23.0 & 23.0 & 919.70 & 919.66 & 49 & $1,174.88$ & $1,175,03$ & $5,376.76$ & 51.10 \\
\hline & 18.0 & 18.0 & 21.8 & 23.0 & 23.0 & & & & & & & \\
\hline & 18.0 & 17.7 & 21.8 & & & & & & & & & \\
\hline & 18.0 & 18.0 & & & 23.0 & & & & & & & 40 \\
\hline & 23.0 & 18.0 & 24.7 & 24.8 & 23.0 & & & & & $1,104.04$ & & .00 \\
\hline & & 18.0 & 25.4 & 25.4 & 25.0 & & & & & 1,29 & $6,078.17$ & 52.00 \\
\hline & & 18.0 & 24 & & 25.0 & & & & & & & \\
\hline & 18.0 & 18.0 & 24 & 27.0 & 25.1 & & & & & & & \\
\hline & 18.0 & 18.0 & 23 & 23.0 & 25.1 & & & 1,0 & & & 63 & \\
\hline & 18.0 & 18.1 & & & 23.0 & & & & & & & \\
\hline & 18.0 & 17.9 & 23.2 & 23.8 & 25.7 & & & & & 1,40 & & \\
\hline & 17.8 & 17.8 & 23.0 & 24.6 & 25.8 & 1,0 & 96 & 1,3 & & & & \\
\hline & 18.0 & 18.0 & 24.3 & 25.8 & 26.0 & & & & & & & \\
\hline & 23.0 & 18.0 & 26. & 23.0 & 26.0 & & & & & & & \\
\hline & 23.0 & 18.0 & 24 & & & & & & & & & \\
\hline & 18.0 & 18.0 & 23 & 22.0 & 23.0 & & & & & & & \\
\hline & 18.0 & 18.0 & 23 & 23.8 & 23.0 & & & & & & & \\
\hline & 18.0 & 18.0 & 24 & 24.0 & 23.0 & & & & & & & \\
\hline & 18.0 & 18.0 & 24 & $23: 0$ & 23.0 & & & & & 1,2 & & \\
\hline & 18.9 & 18.0 & 30 & 37.5 & & & & & & & & \\
\hline & 19.9 & 18.0 & 24 & 23 & 23.1 & & & & & & & \\
\hline & 18 & 14.2 & 22 & & 23.0 & & & & & & & \\
\hline & 8.9 & 14.5 & & & & & & & & 1,1 & & \\
\hline & - & - & $=$ & $\overline{-}$ & $=$ & & & & & & & \\
\hline & 18.9 & 15.9 & & 23. & 23.0 & & & 1,1 & 1,1 & 1,1 & & 51.0 \\
\hline & 19.7 & 19.7 & 30. & & 44.0 & & & & & & & 13.45 \\
\hline & 20.0 & 16.0 & 20. & 20.5 & 23.0 & & & & & & & \\
\hline & 20.0 & 17.9 & 21. & 22.8 & 23.9 & & & & & & & 44 \\
\hline & 17.5 & 18.0 & 21. & 24.9 & 23.0 & & & & & & & 55 \\
\hline & 18.0 & 18.7 & 22.0 & 22.9 & 23.0 & & & & & & & \\
\hline & 18.0 & 18.1 & 34 & 47.6 & 47.3 & & & & & & & \\
\hline & 18.0 & 16.9 & 20.1 & 19. & 24.0 & & & & & & 3, & 37.58 \\
\hline & & & & & & & & & & & & \\
\hline & 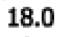 & 17.0 & & & 25.0 & & & & & 1,4 & & \\
\hline & 18.0 & 19.0 & 21. & 20.0 & 27.0 & & & & & & & \\
\hline & 18.0 & 19.0 & & 22 & 23.0 & & & & & & & \\
\hline & 22.1 & 22.9 & & 37.4 & & & & & & & & \\
\hline & 17.5 & 21.1 & 19. & 20.0 & 26.1 & & & 1,0 & 1,0 & 1,3 & & \\
\hline & & 21.0 & & & & & & 1,2 & & & & \\
\hline & & 20.9 & & & 41.5 & & & & & & & \\
\hline & 20.1 & 17.9 & 22.8 & 24.0 & 26.2 & 31 & 06 & 1,41 & 1,4 & 1,6 & 6,8 & \\
\hline & 20.0 & 14.5 & 19 & & & & & & & & & \\
\hline & 21.0 & 20.5 & 28.3 & 40.8 & 31.8 & & & & & & & \\
\hline & 20.0 & 16.0 & 19.4 & 22.4 & 25.7 & & & 1,06 & 1,23 & 1,41 & $5,686,69$ & \\
\hline & 19.5 & 16.4 & 19.7 & & & & 72 & & & & 6,6 & \\
\hline
\end{tabular}


TEM-10200-1

ENGINEERING CALCULATIONS AND ANALYSIS

$12 / 19 / 17$

Rev.08

Title: $\quad$ AS-RUN NEUTRONICS EVALUATION FOR THE GE HITACHI EXPERIMENT IN THE ATR

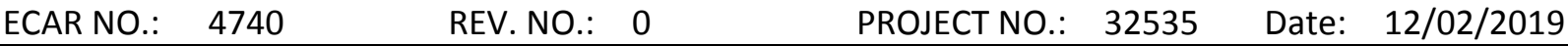

R. R. Little

January 21, 2019

RE-03-19

Page 3 of 5

Table 2. Cumulative Exposure

\begin{tabular}{|c|c|c|c|c|c|c|}
\hline & & & Cumulative $\mathrm{E}$ & sure (MWd) & & \\
\hline & NW & $\mathrm{NE}$ & C & SW & SE & Total \\
\hline 134A-1 & - & - & - & - & - & - \\
\hline $134 A-2$ & 0.21 & 0.30 & 0.39 & 0.36 & 0.36 & 1.62 \\
\hline $134 \mathrm{~B}-1$ & 453.03 & 452.54 & 615.86 & 578.84 & 628.94 & $2,729.21$ \\
\hline $134 \mathrm{~B}-2$ & 838.65 & 838.34 & $1,169.36$ & $1,072.08$ & $1,165.01$ & $5,083.44$ \\
\hline $135 \mathrm{~A}-1$ & $1,074.81$ & $1,074.33$ & $1,560: 43$ & $1,440.71$ & $1,628 ! 99$ & $6,779.27$ \\
\hline 135B-1 & $1,533,09$ & $1,532,68$ & $2,191.22$ & $2,025.98$ & $2,265.50$ & $9,548: 47$ \\
\hline 135B-2 & $1,938.11$ & $1,938.38$ & $2,758.60$ & $2,543.80$ & $2,828.49$ & $12,007.38$ \\
\hline $135 \mathrm{C}-1$ & $2,668.02$ & $2,668.08$ & $3,772.05$ & $3,476.80$ & $3,842.35$ & $16,427.30$ \\
\hline $136 \mathrm{~A}-1$ & $3,584.20$ & $3,584.47$ & $4,990.96$ & $4,646.27$ & $5,012.52$ & $21,818.42$ \\
\hline $136 \mathrm{~B}-1$ & $4,286.14$ & $4,286.79$ & $5,922: 15$ & $5,543.20$ & $5,909: 49$ & $25,947,77$ \\
\hline $137 \mathrm{~A}-1$ & $5,261: 49$ & $5,261.55$ & $7,258: 31$ & $6,626.40$ & $7,262,80$ & $31,670: 55$ \\
\hline $137 \mathrm{~B}-1$ & $5,503.46$ & $5,478.56$ & $7,687: 93$ & $7,311.78$ & $7,630.43$ & $33,612.16$ \\
\hline 138A-1 & $6,550.33$ & $6,526.25$ & $9,058.62$ & $8,648.17$ & $9,083.90$ & $39,867.27$ \\
\hline 138B-1 & $7,388.87$ & $7,365.87$ & $10,143.41$ & $9,719.11$ & $10,248.54$ & $44,865.80$ \\
\hline 139A-1 & $8,317.60$ & $8,294.96$ & $11,374.48$ & $10,905.38$ & $11,538.01$ & $50,430.43$ \\
\hline 139B-1 & $9,237.30$ & $9,214.62$ & $12,561.97$ & $12,080.26$ & $12,713.04$ & $55,807.19$ \\
\hline $140 A-1$ & $10,074.29$ & $10,051.80$ & $13,574.86$ & $13,149,95$ & $13,782.43$ & $60,633.33$ \\
\hline $140 B-1$ & $10,716.01$ & $10,681.29$ & $14,352.06$ & $13,992: 81$ & $14,602,43$ & $64,344,60$ \\
\hline 141A-1 & $11,299.26$ & $11,264.37$ & $15,108.89$ & $14,737.86$ & $15,347.88$ & $67,758.26$ \\
\hline $142 \mathrm{~A}-1$ & $12,404.17$ & $12,129.12$ & $16,294.93$ & $15,930.35$ & $16,451.92$ & $73,210.49$ \\
\hline 142B-1 & $13,601.10$ & $13,065.80$ & $17,618.34$ & $17,252.80$ & $17,750.62$ & $79,288.66$ \\
\hline $143 A-1 / 2$ & $14,481.08$ & $13,948.32$ & $18,806.01$ & $18,568.24$ & $18,973.70$ & $84,777.35$ \\
\hline 143B-1 & $15,513.14$ & $14,980.90$ & $20,229,54$ & $20 ; 111.98$ & $20,408.71$. & $91,244,27$ \\
\hline $144 A-1$ & $16,300.11$ & $15,767.92$ & $21,236.21$ & $21,116.51$ & $21,502.15$ & $95,922,90$ \\
\hline $1448-1$ & $17,232.36$ & $16,701.35$ & $22,391.95$ & $22,307.23$ & $22,693.04$ & $101,325.93$ \\
\hline $145 \mathrm{~A}-1$ & $18,215.33$ & $17,682.24$ & $23,659.29$ & $23,606.72$ & $24,100.79$ & $107,264.37$ \\
\hline 145B-1 & $19,235.79$ & $18,702.20$ & $24,980.72$ & $25,014.51$ & $25,579.04$ & $113,512.26$ \\
\hline $146 \mathrm{~A}-1$ & $20,142.55$ & $19,609.00$ & $26,206.46$ & $26,314.53$ & $26,891.59$ & $119 ; 164.13$ \\
\hline $146 \mathrm{~B}-1$ & $21,046.23$ & $20,316.11$ & $27,228.05$ & $27,218.38$ & $27,912.55$ & $123,721.32$ \\
\hline 147A-1 & $22,203.09$ & $21 ; 220.53$ & $28,436.46$ & $28,267.80$ & $29,067.70$ & $129,195.58$ \\
\hline $148 \mathrm{~A}-1$ & $23,059.06$ & $22,076.28$ & $29,557.89$ & $29,311.59$ & $30,161.34$ & $134,166.16$ \\
\hline $148 \mathrm{~B}-1$ & $23,986.56$ & $23,003.00$ & $30,739.49$ & $30,535.57$ & $31,346.37$ & $139,610.99$ \\
\hline 149A-1 & $24,649.01$ & $23,665.65$ & $31,630.77$ & $31,418.85$ & $32,193.17$ & $143,557.45$ \\
\hline 149B-1 & $25,613.39$ & $24,630.10$ & $32,928.54$ & $32,650,48$ & $33,423.95$ & $149,246.46$ \\
\hline $150 A-1$ & $25,846.80$ & $24,851.70$ & $33,304.47$ & $33,113.19$ & $33,856.53$ & $150,972.69$ \\
\hline 150B-1 & $26,679.64$ & $25,606.42$ & $34,319.05$ & $34,078.05$ & $34,822.53$ & $155,505,69$ \\
\hline $151 \mathrm{~A}-1$ & $27,738.27$ & $26,406.37$ & $35,556.06$ & $35,402.44$ & $36,111.57$ & $161,214.71$ \\
\hline $151 \mathrm{~B}-1 / 2$ & $28,709.68$ & $27,148.12$ & $36,690.93$ & $36,584.38$ & $37,291.54$ & $166,424.65$ \\
\hline $152 A-1 / 6$ & $28,709.98$ & $27,148.42$ & $36,691.43$ & $36,584.78$ & $37,292.04$ & $166,426.65$ \\
\hline 152B-1 & $29,676.40$ & $27,961.42$ & $37,832.71$ & $37,756.86$ & $38,465.61$ & $171,693,00$ \\
\hline 153B-1 & $29,941.60$ & $28,226.77$ & $38,247.18$ & $38,232: 98$ & $39 ; 057,03$ & $173 / 705.56$ \\
\hline 154A-1 & $30,989.72$ & $29,065.37$ & $39,316.56$ & $39,304,40$ & $40,261.25$ & $178,937.30$ \\
\hline 154B-1 & $32,056.32$ & $30,023.84$ & $40,472.96$ & $40,524.89$ & $41,536.52$ & $184,614.53$ \\
\hline 155A-1 & $33,020.78$ & $31,015.89$ & $41,642.60$ & $41,898.04$ & $42,805.85$ & $190,383.16$ \\
\hline 155B-1 & $33,917.19$ & $31,949.67$ & $42,740.05$ & $43,043.32$ & $43,952.06$ & $195,602.29$ \\
\hline $156 \mathrm{~A}-1$ & $34,176.18$ & $32,209: 77$ & $43,236.00$ & $43,729.17$ & $44,633: 83$ & $197,984.95$ \\
\hline $157 \mathrm{~A}-1$ & $34,850.98$ & $32,846.70$ & $43,991.89$ & $44,478.54$ & $45,534.13$ & $201,702.24$ \\
\hline $157 \mathrm{C}-1$ & $34,947.59$ & $32,938.17$ & $44,105: 04$ & $44,586.44$ & $45,668.20$ & $202,245 / 44$ \\
\hline
\end{tabular}


TEM-10200-1

$12 / 19 / 17$

Rev.08

Title: $\quad$ AS-RUN NEUTRONICS EVALUATION FOR THE GE HITACHI EXPERIMENT IN THE ATR

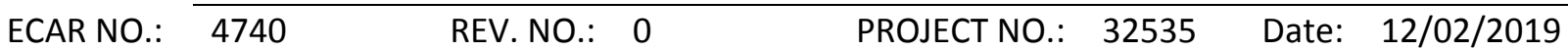

\author{
R. R. Little \\ January 21,2019 \\ RE-03-19 \\ Page 4 of 5
}

Table 2. Cumulative Exposure

\begin{tabular}{|c|c|c|c|c|c|c|}
\hline & \multicolumn{6}{|c|}{ Cumulative Exposure (MWd) } \\
\hline & NW & NE & C & sw & SE & Total \\
\hline 157D-1 & $36,019.65$ & $33,950.68$ & $45,393.20$ & $45,833.58$ & $47,158.51$ & $208,355.62$ \\
\hline 158A-1 & $36,960.97$ & $34,944.84$ & $46,505.28$ & $46,877.74$ & $48,568.56$ & $213,857.39$ \\
\hline 158B-1 & $37,885.82$ & $35,921.84$ & $47,479.61$ & $48,042.99$ & $49,751.90$ & $219,082.16$ \\
\hline $159 A-1$ & $37,954,37$ & 35,99292 & $47,569,75$ & $48,159,00$ & 4986 & 21995655 \\
\hline $160 \mathrm{~A}-1$ & 38,87844 & 37,10888 & $48 ; 620: 46$ & $49,216: 87$ & $51,241,22$ & $225,065.85$ \\
\hline $160 \mathrm{~B}-1$ & $39,958: 49$ & $38,368: 71$ & $49,873.01$ & 50,5 & 52,77887 & $231,576: 06$ \\
\hline $161 \mathrm{~A}-1$ & $40,188.70$ & $38,636.88$ & $50,265.92$ & $51,144.85$ & $53,311.40$ & $233,547.75$ \\
\hline $162 \mathrm{~A}-1$ & $41,433.01$ & $39,741.94$ & $51,676.70$ & $52,630.97$ & $54,932.96$ & $240,415.58$ \\
\hline $162 \mathrm{~B}-1$ & $42,202.05$ & $40,301.99$ & $52,415.68$ & $53,549.81$ & $55,817.86$ & $244,287.39$ \\
\hline $16 B A-1$ & 42,26489 & $40,363.21$ & 52,5 & & 55,91299 & $244 / 713.19$ \\
\hline $164 A-1$ & $43,363,95$ & $41,242,49$ & $53,568,20$ & 54,901174 & $57,323.5$ & 250,39988 \\
\hline 16 & $44,610.22$ & $42,294.21$ & $54,829,78$ & $56,379.54$ & $58,931.32$ & $257,045.07$ \\
\hline
\end{tabular}

Prepared by
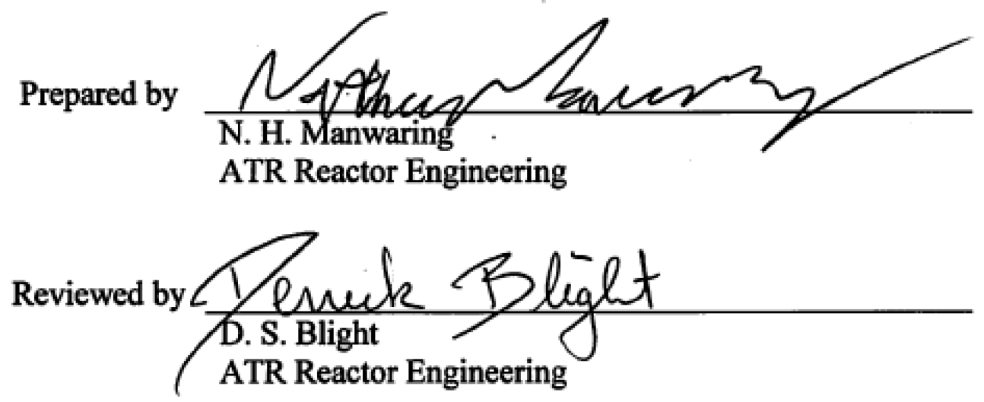
TEM-10200-1

$12 / 19 / 17$

Rev.08

Title: $\quad$ AS-RUN NEUTRONICS EVALUATION FOR THE GE HITACHI EXPERIMENT IN THE ATR

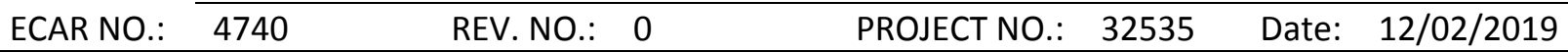

\section{R. R. Little}

January 21, 2019

RE-03-19

Page 5 of 5

cc:

J. O. Brower, MS 3890

H. D. Buckland, MS 7114

R. R. Bullock, MS 7117

K. H. Carlquist, MS 7104

C. D. Cooper, MS 3890

C. C. Dwight, MS 6122

K. R. Estes, MS 7114

B. J. Good, MS 7136

M. J. Guzman, MS 7117

S. A. Hill, MS 7106

M. R. Holtz, MS 7136

R. C. Howard, MS 7101

C. D. Jackson, MS 7106

N. H. Manwaring, MS 7136

C. A. McPhie, FMP, MS 7103

R. M. Murphy, MS 9202

D. R. Norman, 7136

D. Ogden, MS 3553

S. O'Kelly, MS 7117

D. G. Robinson, MS 7104

D. L. Rowsell, MS 7104

D. J. Schoonen, MS 7117

K. A. Smith, MS 7101

L. D. Smith, MS 7111

W. F. Jones, MS 3818

K. D. Stueve, MS 7117

E. T. Swain, MS 3818

T. L. Julius, MS 7104

V. C. Kirkpatrick, MS 7106

D. J. Utterbeck, MS 3870

K. L. Zimmer, MS 7106

M. A. Lillo, MS 3860

ATR Document Management, MS 7116,

R. R. Little, MS 7136

email: Jennifer.eversull@inl.gov

S. G. Louk, MS 7111

N. J. Lybeck, MS 3818

Cycle 164B-1 Surveillance File

Reactor Engineering Letter File (RE-03-19)

Uniform File Code: $\underline{8153}$

Disposition Authority: $\underline{\text { A17-32-b-1 }}$

Retention Schedule: Retain in accordance with current regulatory requirements or for nuclear facilities, 6 years after plant or item is put into operation.

NOTE: Original disposition authority, retention schedule, and Uniform Filing Code applied by the sender may not be appropriate for all recipients. Make adjustments as needed. 


\section{$12 / 19 / 17$}

Rev.08

Title: AS-RUN NEUTRONICS EVALUATION FOR THE GE HITACHI EXPERIMENT IN THE ATR

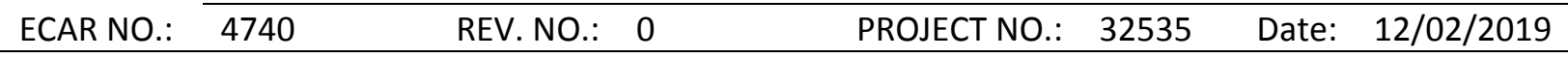

\section{APPENDIX C “ADDITIONAL SOURCE TERM”}

The GE-HITACHI consisted of two sample materials. To support disassembly and future PIE of the GE-HITACHI-10584 experiment the source term for 1 gram of each material scaled to the maximum experiment flux was calculated. This conservative source term can be scaled to the total mass for each sample geometry located in each capsule.

Table 17. Ci/1g of incon

\begin{tabular}{|c|c|c|c|c|c|c|c|c|c|c|}
\hline \multicolumn{11}{|c|}{$1 \mathrm{~g}$ of Inconel } \\
\hline Icoton & EOI & $30.0 \mathrm{MI}$ & $1.0 \mathrm{D}$ & $7.0 \mathrm{D}$ & $30.0 \mathrm{D}$ & $60.0 \mathrm{D}$ & $90.0 \mathrm{D}$ & $120.0 \mathrm{D}$ & $200.0 \mathrm{D}$ & $360.0 \mathrm{D}$ \\
\hline Isotope & $\mathrm{Ci} / 1 \mathrm{~g}$ of incon & $\mathrm{Ci} / 1 \mathrm{~g}$ of incon & $\mathrm{Ci} / 1 \mathrm{~g}$ of incon & $\mathrm{Ci} / 1 \mathrm{~g}$ of incon & $\mathrm{Ci} / 1 \mathrm{~g}$ of incon & $\mathrm{Ci} / 1 \mathrm{~g}$ of incon & $\mathrm{Ci} / 1 \mathrm{~g}$ of incon & $\mathrm{Ci} / 1 \mathrm{~g}$ of incon & $\mathrm{Ci} / 1 \mathrm{~g}$ of incon & $\mathrm{Ci} / 1 \mathrm{~g}$ of incon \\
\hline H 3 & $3.24 \mathrm{E}-10$ & $3.24 \mathrm{E}-10$ & $3.24 \mathrm{E}-10$ & $3.24 \mathrm{E}-10$ & $3.23 \mathrm{E}-10$ & $3.21 \mathrm{E}-10$ & $3.20 \mathrm{E}-10$ & $3.18 \mathrm{E}-10$ & $3.14 \mathrm{E}-10$ & 3.07E-10 \\
\hline $\mathrm{H} 4$ & $7.42 \mathrm{E}-05$ & $0.00 \mathrm{E}+00$ & $0.00 \mathrm{E}+00$ & $0.00 \mathrm{E}+00$ & $0.00 \mathrm{E}+00$ & $0.00 \mathrm{E}+00$ & $0.00 E+00$ & $0.00 \mathrm{E}+00$ & $0.00 E+00$ & $0.00 \mathrm{E}+00$ \\
\hline HE 6 & $8.44 \mathrm{E}-08$ & $0.00 \mathrm{E}+00$ & $0.00 \mathrm{E}+00$ & $0.00 \mathrm{E}+00$ & $0.00 \mathrm{E}+00$ & $0.00 \mathrm{E}+00$ & $0.00 \mathrm{E}+00$ & $0.00 \mathrm{E}+00$ & $0.00 \mathrm{E}+00$ & $0.00 \mathrm{E}+00$ \\
\hline LI 8 & $2.98 \mathrm{E}-05$ & $0.00 \mathrm{E}+00$ & $0.00 \mathrm{E}+00$ & $0.00 \mathrm{E}+00$ & $0.00 E+00$ & $0.00 \mathrm{E}+00$ & $0.00 E+00$ & $0.00 \mathrm{E}+00$ & $0.00 \mathrm{E}+00$ & $0.00 \mathrm{E}+00$ \\
\hline BE 8 & $3.00 E-05$ & $0.00 \mathrm{E}+00$ & $0.00 \mathrm{E}+00$ & $0.00 \mathrm{E}+00$ & $0.00 \mathrm{E}+00$ & $0.00 \mathrm{E}+00$ & $0.00 E+00$ & $0.00 \mathrm{E}+00$ & $0.00 \mathrm{E}+00$ & $0.00 E+00$ \\
\hline BE 10 & $2.83 \mathrm{E}-12$ & $2.83 \mathrm{E}-12$ & $2.83 \mathrm{E}-12$ & $2.83 \mathrm{E}-12$ & $2.83 \mathrm{E}-12$ & $2.83 \mathrm{E}-12$ & $2.83 \mathrm{E}-12$ & $2.83 \mathrm{E}-12$ & $2.83 \mathrm{E}-12$ & $2.83 \mathrm{E}-12$ \\
\hline BE 11 & $1.00 \mathrm{E}-11$ & $0.00 \mathrm{E}+00$ & $0.00 \mathrm{E}+00$ & $0.00 \mathrm{E}+00$ & $0.00 \mathrm{E}+00$ & $0.00 \mathrm{E}+00$ & $0.00 \mathrm{E}+00$ & $0.00 \mathrm{E}+00$ & $0.00 \mathrm{E}+00$ & $0.00 \mathrm{E}+00$ \\
\hline B 12 & $2.05 \mathrm{E}-05$ & $0.00 \mathrm{E}+00$ & $0.00 \mathrm{E}+00$ & $0.00 \mathrm{E}+00$ & $0.00 \mathrm{E}+00$ & $0.00 \mathrm{E}+00$ & $0.00 E+00$ & $0.00 \mathrm{E}+00$ & $0.00 \mathrm{E}+00$ & $0.00 \mathrm{E}+00$ \\
\hline C 14 & $4.85 \mathrm{E}-11$ & $4.85 \mathrm{E}-11$ & $4.85 \mathrm{E}-11$ & $4.85 \mathrm{E}-11$ & $4.85 \mathrm{E}-11$ & $4.85 \mathrm{E}-11$ & $4.85 \mathrm{E}-11$ & $4.85 \mathrm{E}-11$ & $4.85 \mathrm{E}-11$ & $4.85 \mathrm{E}-11$ \\
\hline NE 23 & $9.05 \mathrm{E}-10$ & $2.55 \mathrm{E}-24$ & $0.00 \mathrm{E}+00$ & $0.00 \mathrm{E}+00$ & $0.00 \mathrm{E}+00$ & $0.00 \mathrm{E}+00$ & $0.00 \mathrm{E}+00$ & $0.00 \mathrm{E}+00$ & $0.00 \mathrm{E}+00$ & $0.00 \mathrm{E}+00$ \\
\hline NA 24 & 5.07E-04 & $4.95 \mathrm{E}-04$ & 1.67E-04 & $2.15 \mathrm{E}-07$ & $1.80 \mathrm{E}-18$ & $0.00 \mathrm{E}+00$ & $0.00 \mathrm{E}+00$ & $0.00 \mathrm{E}+00$ & $0.00 \mathrm{E}+00$ & $0.00 \mathrm{E}+00$ \\
\hline NA 24M & $1.73 \mathrm{E}-13$ & $0.00 \mathrm{E}+00$ & $0.00 \mathrm{E}+00$ & $0.00 \mathrm{E}+00$ & $0.00 \mathrm{E}+00$ & $0.00 \mathrm{E}+00$ & $0.00 \mathrm{E}+00$ & $0.00 \mathrm{E}+00$ & $0.00 \mathrm{E}+00$ & $0.00 \mathrm{E}+00$ \\
\hline NA 25 & $1.37 \mathrm{E}-09$ & $1.11 \mathrm{E}-18$ & $0.00 \mathrm{E}+00$ & $0.00 \mathrm{E}+00$ & $0.00 \mathrm{E}+00$ & $0.00 \mathrm{E}+00$ & $0.00 \mathrm{E}+00$ & $0.00 \mathrm{E}+00$ & $0.00 \mathrm{E}+00$ & $0.00 \mathrm{E}+00$ \\
\hline MG 27 & $2.42 \mathrm{E}-03$ & $2.69 \mathrm{E}-04$ & $0.00 \mathrm{E}+00$ & $0.00 \mathrm{E}+00$ & $0.00 \mathrm{E}+00$ & $0.00 \mathrm{E}+00$ & $0.00 \mathrm{E}+00$ & $0.00 \mathrm{E}+00$ & $0.00 \mathrm{E}+00$ & $0.00 \mathrm{E}+00$ \\
\hline MG 28 & $3.85 \mathrm{E}-12$ & $3.79 \mathrm{E}-12$ & $1.74 \mathrm{E}-12$ & $1.47 \mathrm{E}-14$ & $1.66 \mathrm{E}-22$ & $0.00 \mathrm{E}+00$ & $0.00 \mathrm{E}+00$ & $0.00 \mathrm{E}+00$ & $0.00 \mathrm{E}+00$ & $0.00 \mathrm{E}+00$ \\
\hline AL 28 & $5.93 \mathrm{E}-02$ & $5.51 \mathrm{E}-06$ & $1.74 \mathrm{E}-12$ & 1.47E-14 & $1.67 \mathrm{E}-22$ & $0.00 \mathrm{E}+00$ & $0.00 \mathrm{E}+00$ & $0.00 \mathrm{E}+00$ & $0.00 \mathrm{E}+00$ & $0.00 \mathrm{E}+00$ \\
\hline AL 29 & $4.16 \mathrm{E}-05$ & $1.71 \mathrm{E}-06$ & $0.00 \mathrm{E}+00$ & $0.00 \mathrm{E}+00$ & $0.00 \mathrm{E}+00$ & $0.00 \mathrm{E}+00$ & $0.00 \mathrm{E}+00$ & $0.00 \mathrm{E}+00$ & $0.00 \mathrm{E}+00$ & $0.00 \mathrm{E}+00$ \\
\hline AL 30 & $4.02 \mathrm{E}-08$ & $0.00 \mathrm{E}+00$ & $0.00 \mathrm{E}+00$ & $0.00 \mathrm{E}+00$ & $0.00 \mathrm{E}+00$ & $0.00 \mathrm{E}+00$ & $0.00 \mathrm{E}+00$ & $0.00 \mathrm{E}+00$ & $0.00 \mathrm{E}+00$ & $0.00 \mathrm{E}+00$ \\
\hline SI 31 & $7.71 \mathrm{E}-04$ & $6.76 \mathrm{E}-04$ & $1.35 \mathrm{E}-06$ & $3.95 \mathrm{E}-23$ & $0.00 \mathrm{E}+00$ & $0.00 \mathrm{E}+00$ & $0.00 \mathrm{E}+00$ & $0.00 \mathrm{E}+00$ & $0.00 \mathrm{E}+00$ & $0.00 \mathrm{E}+00$ \\
\hline SI 32 & $1.57 \mathrm{E}-13$ & $1.57 \mathrm{E}-13$ & 1.57E-13 & $1.57 \mathrm{E}-13$ & 1.57E-13 & $1.57 \mathrm{E}-13$ & $1.57 \mathrm{E}-13$ & $1.57 \mathrm{E}-13$ & 1.57E-13 & 1.57E-13 \\
\hline P 32 & $1.39 \mathrm{E}-03$ & $1.39 \mathrm{E}-03$ & $1.33 \mathrm{E}-03$ & $9.91 \mathrm{E}-04$ & $3.25 \mathrm{E}-04$ & $7.59 \mathrm{E}-05$ & $1.77 \mathrm{E}-05$ & $4.14 \mathrm{E}-06$ & $8.57 \mathrm{E}-08$ & $3.69 \mathrm{E}-11$ \\
\hline P 33 & $5.82 \mathrm{E}-08$ & $5.82 \mathrm{E}-08$ & $5.66 \mathrm{E}-08$ & $4.79 \mathrm{E}-08$ & $2.53 \mathrm{E}-08$ & $1.10 \mathrm{E}-08$ & $4.80 \mathrm{E}-09$ & $2.09 \mathrm{E}-09$ & $2.27 \mathrm{E}-10$ & $2.69 \mathrm{E}-12$ \\
\hline P 34 & $3.27 \mathrm{E}-07$ & $0.00 \mathrm{E}+00$ & $0.00 \mathrm{E}+00$ & $0.00 \mathrm{E}+00$ & $0.00 \mathrm{E}+00$ & $0.00 \mathrm{E}+00$ & $0.00 E+00$ & $0.00 \mathrm{E}+00$ & $0.00 \mathrm{E}+00$ & $0.00 \mathrm{E}+00$ \\
\hline
\end{tabular}


TEM-10200-1

$12 / 19 / 17$

Rev.08

Title:

AS-RUN NEUTRONICS EVALUATION FOR THE GE HITACHI EXPERIMENT IN THE ATR

\begin{tabular}{llllllll} 
ECAR NO.: & 4740 & REV. NO.: & 0 & PROJECT NO.: & 32535 & Date: & $12 / 02 / 2019$ \\
\hline
\end{tabular}

\begin{tabular}{|c|c|c|c|c|c|c|c|c|c|c|}
\hline \multicolumn{11}{|c|}{$1 \mathrm{~g}$ of Inconel } \\
\hline Icton & EOI & $30.0 \mathrm{MI}$ & $1.0 \mathrm{D}$ & $7.0 \mathrm{D}$ & $30.0 \mathrm{D}$ & $60.0 \mathrm{D}$ & $90.0 \mathrm{D}$ & $120.0 \mathrm{D}$ & $200.0 \mathrm{D}$ & $360.0 \mathrm{D}$ \\
\hline Isotope & $\mathrm{Ci} / 1 \mathrm{~g}$ of incon & $\mathrm{Ci} / 1 \mathrm{~g}$ of incon & $\mathrm{Ci} / 1 \mathrm{~g}$ of incon & $\mathrm{Ci} / 1 \mathrm{~g}$ of incon & $\mathrm{Ci} / 1 \mathrm{~g}$ of incon & $\mathrm{Ci} / 1 \mathrm{~g}$ of incon & $\mathrm{Ci} / 1 \mathrm{~g}$ of incon & $\mathrm{Ci} / 1 \mathrm{~g}$ of incon & $\mathrm{Ci} / 1 \mathrm{~g}$ of incon & $\mathrm{Ci} / 1 \mathrm{~g}$ of incon \\
\hline S 35 & $3.08 \mathrm{E}-05$ & $3.08 \mathrm{E}-05$ & $3.05 \mathrm{E}-05$ & $2.91 \mathrm{E}-05$ & 2.43E-05 & $1.92 \mathrm{E}-05$ & 1.51E-05 & $1.20 \mathrm{E}-05$ & $6.36 \mathrm{E}-06$ & $1.81 \mathrm{E}-06$ \\
\hline S 37 & $9.43 \mathrm{E}-08$ & $1.55 \mathrm{E}-09$ & $0.00 \mathrm{E}+00$ & $0.00 \mathrm{E}+00$ & $0.00 \mathrm{E}+00$ & $0.00 \mathrm{E}+00$ & $0.00 \mathrm{E}+00$ & $0.00 \mathrm{E}+00$ & $0.00 \mathrm{E}+00$ & $0.00 \mathrm{E}+00$ \\
\hline CL 36 & $3.92 \mathrm{E}-13$ & $3.92 \mathrm{E}-13$ & $3.92 \mathrm{E}-13$ & $3.92 \mathrm{E}-13$ & $3.92 \mathrm{E}-13$ & $3.92 \mathrm{E}-13$ & $3.92 \mathrm{E}-13$ & $3.92 \mathrm{E}-13$ & $3.92 \mathrm{E}-13$ & $3.92 \mathrm{E}-13$ \\
\hline CL 38 & $4.02 \mathrm{E}-11$ & $2.30 \mathrm{E}-11$ & $9.05 E-23$ & $0.00 \mathrm{E}+00$ & $0.00 \mathrm{E}+00$ & $0.00 \mathrm{E}+00$ & $0.00 E+00$ & $0.00 \mathrm{E}+00$ & $0.00 \mathrm{E}+00$ & $0.00 \mathrm{E}+00$ \\
\hline CL 38M & $4.79 \mathrm{E}-13$ & $0.00 \mathrm{E}+00$ & $0.00 \mathrm{E}+00$ & $0.00 \mathrm{E}+00$ & $0.00 \mathrm{E}+00$ & $0.00 \mathrm{E}+00$ & $0.00 \mathrm{E}+00$ & $0.00 \mathrm{E}+00$ & $0.00 \mathrm{E}+00$ & $0.00 \mathrm{E}+00$ \\
\hline AR 41 & $2.81 \mathrm{E}-11$ & $2.33 \mathrm{E}-11$ & $3.12 \mathrm{E}-15$ & $0.00 \mathrm{E}+00$ & $0.00 \mathrm{E}+00$ & $0.00 \mathrm{E}+00$ & $0.00 \mathrm{E}+00$ & $0.00 \mathrm{E}+00$ & $0.00 \mathrm{E}+00$ & $0.00 \mathrm{E}+00$ \\
\hline K 42 & $2.29 \mathrm{E}-13$ & $2.22 \mathrm{E}-13$ & $5.95 \mathrm{E}-14$ & $1.86 \mathrm{E}-17$ & $3.58 \mathrm{E}-20$ & $3.58 \mathrm{E}-20$ & $3.58 \mathrm{E}-20$ & $3.58 \mathrm{E}-20$ & $3.58 \mathrm{E}-20$ & $3.58 \mathrm{E}-20$ \\
\hline K 43 & $3.57 \mathrm{E}-10$ & $3.51 \mathrm{E}-10$ & $1.71 \mathrm{E}-10$ & $2.06 \mathrm{E}-12$ & $9.16 \mathrm{E}-20$ & $2.35 \mathrm{E}-29$ & $0.00 \mathrm{E}+00$ & $0.00 \mathrm{E}+00$ & $0.00 \mathrm{E}+00$ & $0.00 \mathrm{E}+00$ \\
\hline K 44 & $6.36 \mathrm{E}-11$ & $2.47 \mathrm{E}-11$ & $0.00 \mathrm{E}+00$ & $0.00 \mathrm{E}+00$ & $0.00 \mathrm{E}+00$ & $0.00 \mathrm{E}+00$ & $0.00 \mathrm{E}+00$ & $0.00 \mathrm{E}+00$ & $0.00 \mathrm{E}+00$ & $0.00 \mathrm{E}+00$ \\
\hline CA 45 & $3.20 \mathrm{E}-06$ & $3.20 \mathrm{E}-06$ & $3.19 \mathrm{E}-06$ & $3.11 \mathrm{E}-06$ & $2.82 \mathrm{E}-06$ & $2.48 \mathrm{E}-06$ & $2.18 \mathrm{E}-06$ & $1.92 \mathrm{E}-06$ & $1.37 \mathrm{E}-06$ & $6.92 \mathrm{E}-07$ \\
\hline CA 47 & $3.14 \mathrm{E}-08$ & $3.13 \mathrm{E}-08$ & $2.70 \mathrm{E}-08$ & $1.08 \mathrm{E}-08$ & $3.21 \mathrm{E}-10$ & $3.28 \mathrm{E}-12$ & 3.34E-14 & $3.42 \mathrm{E}-16$ & $1.68 \mathrm{E}-21$ & $0.00 \mathrm{E}+00$ \\
\hline SC 46 & $5.60 \mathrm{E}-04$ & $5.59 \mathrm{E}-04$ & $5.55 \mathrm{E}-04$ & $5.28 \mathrm{E}-04$ & 4.37E-04 & $3.41 \mathrm{E}-04$ & $2.66 \mathrm{E}-04$ & 2.07E-04 & 1.07E-04 & $2.85 \mathrm{E}-05$ \\
\hline SC 46M & $1.59 \mathrm{E}-08$ & $0.00 \mathrm{E}+00$ & $0.00 \mathrm{E}+00$ & $0.00 \mathrm{E}+00$ & $0.00 \mathrm{E}+00$ & $0.00 \mathrm{E}+00$ & $0.00 \mathrm{E}+00$ & $0.00 \mathrm{E}+00$ & $0.00 \mathrm{E}+00$ & $0.00 \mathrm{E}+00$ \\
\hline SC 47 & $1.04 \mathrm{E}-03$ & $1.03 \mathrm{E}-03$ & 8.44E-04 & $2.44 \mathrm{E}-04$ & $2.10 \mathrm{E}-06$ & 4.24E-09 & $8.68 \mathrm{E}-12$ & $1.86 \mathrm{E}-14$ & $7.55 \mathrm{E}-21$ & $0.00 \mathrm{E}+00$ \\
\hline SC 48 & $9.72 \mathrm{E}-05$ & $9.65 \mathrm{E}-05$ & $6.65 \mathrm{E}-05$ & $6.81 \mathrm{E}-06$ & 1.10E-09 & $1.24 \mathrm{E}-14$ & $1.39 \mathrm{E}-19$ & $1.57 \mathrm{E}-24$ & $0.00 \mathrm{E}+00$ & $0.00 \mathrm{E}+00$ \\
\hline SC 49 & $8.62 \mathrm{E}-05$ & $6.01 \mathrm{E}-05$ & $2.49 \mathrm{E}-12$ & $0.00 \mathrm{E}+00$ & $0.00 \mathrm{E}+00$ & $0.00 \mathrm{E}+00$ & $0.00 \mathrm{E}+00$ & $0.00 \mathrm{E}+00$ & $0.00 \mathrm{E}+00$ & $0.00 \mathrm{E}+00$ \\
\hline SC 50 & $1.65 \mathrm{E}-06$ & $8.53 \mathrm{E}-12$ & $0.00 \mathrm{E}+00$ & $0.00 \mathrm{E}+00$ & $0.00 \mathrm{E}+00$ & $0.00 \mathrm{E}+00$ & $0.00 \mathrm{E}+00$ & $0.00 \mathrm{E}+00$ & $0.00 \mathrm{E}+00$ & $0.00 \mathrm{E}+00$ \\
\hline TI 51 & $1.88 \mathrm{E}-03$ & $5.10 \mathrm{E}-05$ & $0.00 \mathrm{E}+00$ & $0.00 \mathrm{E}+00$ & $0.00 \mathrm{E}+00$ & $0.00 \mathrm{E}+00$ & $0.00 \mathrm{E}+00$ & $0.00 \mathrm{E}+00$ & $0.00 \mathrm{E}+00$ & $0.00 \mathrm{E}+00$ \\
\hline V 52 & $2.20 \mathrm{E}-02$ & $8.61 \mathrm{E}-05$ & $0.00 \mathrm{E}+00$ & $0.00 \mathrm{E}+00$ & $0.00 \mathrm{E}+00$ & $0.00 \mathrm{E}+00$ & $0.00 \mathrm{E}+00$ & $0.00 \mathrm{E}+00$ & $0.00 \mathrm{E}+00$ & $0.00 \mathrm{E}+00$ \\
\hline V 53 & $1.02 \mathrm{E}-04$ & $2.50 \mathrm{E}-10$ & $0.00 \mathrm{E}+00$ & $0.00 \mathrm{E}+00$ & $0.00 \mathrm{E}+00$ & $0.00 \mathrm{E}+00$ & $0.00 \mathrm{E}+00$ & $0.00 \mathrm{E}+00$ & $0.00 \mathrm{E}+00$ & $0.00 \mathrm{E}+00$ \\
\hline V 54 & $1.07 \mathrm{E}-06$ & $1.51 \mathrm{E}-16$ & $0.00 \mathrm{E}+00$ & $0.00 \mathrm{E}+00$ & $0.00 \mathrm{E}+00$ & $0.00 \mathrm{E}+00$ & $0.00 \mathrm{E}+00$ & $0.00 \mathrm{E}+00$ & $0.00 \mathrm{E}+00$ & $0.00 \mathrm{E}+00$ \\
\hline CR 51 & $2.19 \mathrm{E}+00$ & $2.19 \mathrm{E}+00$ & $2.14 \mathrm{E}+00$ & $1.84 \mathrm{E}+00$ & $1.03 \mathrm{E}+00$ & $4.88 \mathrm{E}-01$ & $2.30 \mathrm{E}-01$ & $1.09 \mathrm{E}-01$ & 1.47E-02 & $2.69 \mathrm{E}-04$ \\
\hline CR 55 & $2.96 \mathrm{E}-02$ & $8.45 \mathrm{E}-05$ & $0.00 \mathrm{E}+00$ & $0.00 \mathrm{E}+00$ & $0.00 \mathrm{E}+00$ & $0.00 \mathrm{E}+00$ & $0.00 \mathrm{E}+00$ & $0.00 \mathrm{E}+00$ & $0.00 \mathrm{E}+00$ & $0.00 \mathrm{E}+00$ \\
\hline MN 54 & $7.71 \mathrm{E}-03$ & $7.71 \mathrm{E}-03$ & $7.69 \mathrm{E}-03$ & $7.59 \mathrm{E}-03$ & $7.21 \mathrm{E}-03$ & $6.75 \mathrm{E}-03$ & $6.31 \mathrm{E}-03$ & $5.90 \mathrm{E}-03$ & $4.94 \mathrm{E}-03$ & $3.47 \mathrm{E}-03$ \\
\hline MN 56 & $9.51 \mathrm{E}-01$ & $8.32 \mathrm{E}-01$ & $1.50 \mathrm{E}-03$ & $2.32 \mathrm{E}-20$ & $0.00 \mathrm{E}+00$ & $0.00 \mathrm{E}+00$ & $0.00 \mathrm{E}+00$ & $0.00 \mathrm{E}+00$ & $0.00 \mathrm{E}+00$ & $0.00 \mathrm{E}+00$ \\
\hline MN 57 & $5.03 \mathrm{E}-05$ & $1.24 \mathrm{E}-10$ & $0.00 \mathrm{E}+00$ & $0.00 \mathrm{E}+00$ & $0.00 \mathrm{E}+00$ & $0.00 \mathrm{E}+00$ & $0.00 \mathrm{E}+00$ & $0.00 \mathrm{E}+00$ & $0.00 \mathrm{E}+00$ & $0.00 \mathrm{E}+00$ \\
\hline MN 58 & $1.66 \mathrm{E}-07$ & $8.36 \mathrm{E}-16$ & $0.00 \mathrm{E}+00$ & $0.00 \mathrm{E}+00$ & $0.00 \mathrm{E}+00$ & $0.00 \mathrm{E}+00$ & $0.00 \mathrm{E}+00$ & $0.00 \mathrm{E}+00$ & $0.00 \mathrm{E}+00$ & $0.00 \mathrm{E}+00$ \\
\hline FE 55 & $4.22 \mathrm{E}-02$ & $4.22 \mathrm{E}-02$ & $4.21 \mathrm{E}-02$ & $4.19 \mathrm{E}-02$ & $4.12 \mathrm{E}-02$ & $4.03 \mathrm{E}-02$ & $3.95 \mathrm{E}-02$ & $3.86 \mathrm{E}-02$ & $3.64 \mathrm{E}-02$ & $3.24 \mathrm{E}-02$ \\
\hline FE 59 & $6.42 \mathrm{E}-03$ & $6.41 \mathrm{E}-03$ & $6.32 \mathrm{E}-03$ & $5.76 \mathrm{E}-03$ & $4.04 \mathrm{E}-03$ & $2.55 \mathrm{E}-03$ & 1.60E-03 & $1.01 \mathrm{E}-03$ & $2.95 \mathrm{E}-04$ & $2.51 \mathrm{E}-05$ \\
\hline CO 58 & $4.57 \mathrm{E}-01$ & 4.57E-01 & $4.53 \mathrm{E}-01$ & 4.27E-01 & $3.41 \mathrm{E}-01$ & $2.54 \mathrm{E}-01$ & $1.89 \mathrm{E}-01$ & $1.41 \mathrm{E}-01$ & $6.45 \mathrm{E}-02$ & $1.35 \mathrm{E}-02$ \\
\hline CO 60 & $5.25 \mathrm{E}-01$ & $5.25 \mathrm{E}-01$ & $5.25 \mathrm{E}-01$ & $5.23 \mathrm{E}-01$ & $5.19 \mathrm{E}-01$ & 5.14E-01 & $5.08 \mathrm{E}-01$ & $5.03 \mathrm{E}-01$ & $4.88 \mathrm{E}-01$ & $4.61 \mathrm{E}-01$ \\
\hline CO 60M & $3.65 \mathrm{E}+00$ & $5.02 \mathrm{E}-01$ & $0.00 \mathrm{E}+00$ & $0.00 \mathrm{E}+00$ & $0.00 \mathrm{E}+00$ & $0.00 \mathrm{E}+00$ & $0.00 \mathrm{E}+00$ & $0.00 \mathrm{E}+00$ & $0.00 \mathrm{E}+00$ & $0.00 \mathrm{E}+00$ \\
\hline CO 61 & $1.83 \mathrm{E}-02$ & $1.49 \mathrm{E}-02$ & $7.66 \mathrm{E}-07$ & $0.00 \mathrm{E}+00$ & $0.00 \mathrm{E}+00$ & $0.00 \mathrm{E}+00$ & $0.00 \mathrm{E}+00$ & $0.00 \mathrm{E}+00$ & $0.00 \mathrm{E}+00$ & $0.00 \mathrm{E}+00$ \\
\hline $\mathrm{CO} 62$ & $9.33 \mathrm{E}-05$ & $8.90 \mathrm{E}-11$ & $0.00 \mathrm{E}+00$ & $0.00 \mathrm{E}+00$ & $0.00 \mathrm{E}+00$ & $0.00 \mathrm{E}+00$ & $0.00 \mathrm{E}+00$ & $0.00 \mathrm{E}+00$ & $0.00 \mathrm{E}+00$ & $0.00 \mathrm{E}+00$ \\
\hline NI 59 & $1.31 \mathrm{E}-04$ & $1.31 \mathrm{E}-04$ & $1.31 \mathrm{E}-04$ & $1.31 \mathrm{E}-04$ & $1.31 \mathrm{E}-04$ & $1.31 \mathrm{E}-04$ & $1.31 \mathrm{E}-04$ & $1.31 \mathrm{E}-04$ & $1.31 \mathrm{E}-04$ & $1.31 \mathrm{E}-04$ \\
\hline NI 63 & $1.69 \mathrm{E}-02$ & $1.69 \mathrm{E}-02$ & $1.69 \mathrm{E}-02$ & $1.69 \mathrm{E}-02$ & $1.69 \mathrm{E}-02$ & $1.69 \mathrm{E}-02$ & $1.69 \mathrm{E}-02$ & $1.69 \mathrm{E}-02$ & $1.68 \mathrm{E}-02$ & $1.68 \mathrm{E}-02$ \\
\hline NI 65 & $1.08 \mathrm{E}-01$ & $9.43 \mathrm{E}-02$ & 1.47E-04 & $9.25 \mathrm{E}-22$ & $0.00 \mathrm{E}+00$ & $0.00 \mathrm{E}+00$ & $0.00 \mathrm{E}+00$ & $0.00 \mathrm{E}+00$ & $0.00 \mathrm{E}+00$ & $0.00 \mathrm{E}+00$ \\
\hline
\end{tabular}


TEM-10200-1

$12 / 19 / 17$

Rev.08

Title:

AS-RUN NEUTRONICS EVALUATION FOR THE GE HITACHI EXPERIMENT IN THE ATR

ECAR NO.: $4740 \quad$ REV. NO.: 0

\begin{tabular}{|c|c|c|c|c|c|c|c|c|c|c|}
\hline \multicolumn{11}{|c|}{$1 \mathrm{~g}$ of Inconel } \\
\hline Icotono & EOI & $30.0 \mathrm{MI}$ & $1.0 \mathrm{D}$ & $7.0 \mathrm{D}$ & $30.0 \mathrm{D}$ & $60.0 \mathrm{D}$ & $90.0 \mathrm{D}$ & $120.0 \mathrm{D}$ & $200.0 \mathrm{D}$ & $360.0 \mathrm{D}$ \\
\hline Isotope & $\mathrm{Ci} / 1 \mathrm{~g}$ of incon & $\mathrm{Ci} / 1 \mathrm{~g}$ of incon & $\mathrm{Ci} / 1 \mathrm{~g}$ of incon & $\mathrm{Ci} / 1 \mathrm{~g}$ of incon & $\mathrm{Ci} / 1 \mathrm{~g}$ of incon & $\mathrm{Ci} / 1 \mathrm{~g}$ of incon & $\mathrm{Ci} / 1 \mathrm{~g}$ of incon & $\mathrm{Ci} / 1 \mathrm{~g}$ of incon & $\mathrm{Ci} / 1 \mathrm{~g}$ of incon & $\mathrm{Ci} / 1 \mathrm{~g}$ of incon \\
\hline NI 66 & $1.65 \mathrm{E}-06$ & 1.64E-06 & $1.22 \mathrm{E}-06$ & $1.96 \mathrm{E}-07$ & 1.77E-10 & $1.91 \mathrm{E}-14$ & $2.05 \mathrm{E}-18$ & $2.20 \mathrm{E}-22$ & $0.00 \mathrm{E}+00$ & $0.00 \mathrm{E}+00$ \\
\hline CU 64 & $1.36 \mathrm{E}-01$ & $1.32 \mathrm{E}-01$ & $3.66 \mathrm{E}-02$ & $1.41 \mathrm{E}-05$ & $1.16 \mathrm{E}-18$ & $0.00 \mathrm{E}+00$ & $0.00 \mathrm{E}+00$ & $0.00 \mathrm{E}+00$ & $0.00 \mathrm{E}+00$ & $0.00 \mathrm{E}+00$ \\
\hline CU 66 & $2.95 \mathrm{E}-02$ & $5.02 \mathrm{E}-04$ & $1.22 \mathrm{E}-06$ & $1.96 \mathrm{E}-07$ & $1.78 \mathrm{E}-10$ & $1.91 \mathrm{E}-14$ & $2.05 \mathrm{E}-18$ & $2.20 \mathrm{E}-22$ & $0.00 \mathrm{E}+00$ & $0.00 \mathrm{E}+00$ \\
\hline CU 67 & $8.55 \mathrm{E}-08$ & $8.50 \mathrm{E}-08$ & $6.53 \mathrm{E}-08$ & $1.30 \mathrm{E}-08$ & $2.67 \mathrm{E}-11$ & $8.37 \mathrm{E}-15$ & $2.63 \mathrm{E}-18$ & $8.23 \mathrm{E}-22$ & $0.00 \mathrm{E}+00$ & $0.00 \mathrm{E}+00$ \\
\hline ZN 65 & $1.26 \mathrm{E}-05$ & $1.26 \mathrm{E}-05$ & $1.26 \mathrm{E}-05$ & $1.24 \mathrm{E}-05$ & $1.16 \mathrm{E}-05$ & $1.06 \mathrm{E}-05$ & $9.75 \mathrm{E}-06$ & $8.96 \mathrm{E}-06$ & $7.13 \mathrm{E}-06$ & $4.53 \mathrm{E}-06$ \\
\hline ZN 69 & $7.72 \mathrm{E}-11$ & $5.51 \mathrm{E}-11$ & 1.66E-12 & $1.18 \mathrm{E}-15$ & $0.00 \mathrm{E}+00$ & $0.00 \mathrm{E}+00$ & $0.00 \mathrm{E}+00$ & $0.00 \mathrm{E}+00$ & $0.00 \mathrm{E}+00$ & $0.00 \mathrm{E}+00$ \\
\hline ZN 69M & $5.19 \mathrm{E}-12$ & $5.06 \mathrm{E}-12$ & $1.55 \mathrm{E}-12$ & $1.10 \mathrm{E}-15$ & $9.22 \mathrm{E}-28$ & $0.00 \mathrm{E}+00$ & $0.00 \mathrm{E}+00$ & $0.00 \mathrm{E}+00$ & $0.00 \mathrm{E}+00$ & $0.00 \mathrm{E}+00$ \\
\hline GA 70 & $1.01 \mathrm{E}-13$ & $3.82 \mathrm{E}-14$ & $0.00 \mathrm{E}+00$ & $0.00 \mathrm{E}+00$ & $0.00 \mathrm{E}+00$ & $0.00 \mathrm{E}+00$ & $0.00 \mathrm{E}+00$ & $0.00 \mathrm{E}+00$ & $0.00 \mathrm{E}+00$ & $0.00 \mathrm{E}+00$ \\
\hline SR 89 & $1.99 \mathrm{E}-10$ & $1.99 \mathrm{E}-10$ & $1.96 \mathrm{E}-10$ & $1.81 \mathrm{E}-10$ & $1.32 \mathrm{E}-10$ & $8.72 \mathrm{E}-11$ & $5.78 \mathrm{E}-11$ & $3.83 \mathrm{E}-11$ & $1.28 \mathrm{E}-11$ & 1.42E-12 \\
\hline SR 90 & $1.02 \mathrm{E}-12$ & $1.02 \mathrm{E}-12$ & $1.02 \mathrm{E}-12$ & $1.02 \mathrm{E}-12$ & $1.01 \mathrm{E}-12$ & $1.01 \mathrm{E}-12$ & $1.01 \mathrm{E}-12$ & $1.01 \mathrm{E}-12$ & 1.00E-12 & $9.92 \mathrm{E}-13$ \\
\hline SR 91 & $5.14 \mathrm{E}-12$ & $4.95 \mathrm{E}-12$ & $8.92 \mathrm{E}-13$ & $2.44 \mathrm{E}-17$ & $0.00 \mathrm{E}+00$ & $0.00 \mathrm{E}+00$ & $0.00 \mathrm{E}+00$ & $0.00 \mathrm{E}+00$ & $0.00 \mathrm{E}+00$ & $0.00 \mathrm{E}+00$ \\
\hline Y 89M & 4.17E-06 & 4.15E-06 & $3.37 E-06$ & 9.45E-07 & $7.20 \mathrm{E}-09$ & $1.24 \mathrm{E}-11$ & $2.14 \mathrm{E}-14$ & $3.89 \mathrm{E}-17$ & 1.67E-24 & $0.00 \mathrm{E}+00$ \\
\hline Y90 & $9.40 \mathrm{E}-05$ & $9.35 \mathrm{E}-05$ & $7.25 \mathrm{E}-05$ & $1.52 \mathrm{E}-05$ & $3.86 \mathrm{E}-08$ & $1.68 \mathrm{E}-11$ & $1.02 \mathrm{E}-12$ & $1.01 \mathrm{E}-12$ & 1.00E-12 & $9.92 \mathrm{E}-13$ \\
\hline Y 90M & $4.10 \mathrm{E}-12$ & $3.66 \mathrm{E}-12$ & $1.92 \mathrm{E}-14$ & $2.00 \mathrm{E}-28$ & $0.00 \mathrm{E}+00$ & $0.00 \mathrm{E}+00$ & $0.00 \mathrm{E}+00$ & $0.00 \mathrm{E}+00$ & $0.00 \mathrm{E}+00$ & $0.00 \mathrm{E}+00$ \\
\hline Y91 & $6.54 \mathrm{E}-09$ & $6.53 \mathrm{E}-09$ & $6.46 \mathrm{E}-09$ & $6.02 \mathrm{E}-09$ & $4.58 \mathrm{E}-09$ & $3.21 \mathrm{E}-09$ & $2.25 \mathrm{E}-09$ & $1.58 \mathrm{E}-09$ & $6.11 \mathrm{E}-10$ & $9.18 \mathrm{E}-11$ \\
\hline Y 92 & $6.42 \mathrm{E}-10$ & $5.82 \mathrm{E}-10$ & $5.84 \mathrm{E}-12$ & $3.29 \mathrm{E}-24$ & $0.00 \mathrm{E}+00$ & $0.00 \mathrm{E}+00$ & $0.00 \mathrm{E}+00$ & $0.00 \mathrm{E}+00$ & $0.00 \mathrm{E}+00$ & $0.00 \mathrm{E}+00$ \\
\hline Y 94 & $3.63 \mathrm{E}-12$ & $1.22 \mathrm{E}-12$ & $0.00 \mathrm{E}+00$ & $0.00 \mathrm{E}+00$ & $0.00 \mathrm{E}+00$ & $0.00 \mathrm{E}+00$ & $0.00 \mathrm{E}+00$ & $0.00 \mathrm{E}+00$ & $0.00 \mathrm{E}+00$ & $0.00 \mathrm{E}+00$ \\
\hline ZR 89 & $4.18 \mathrm{E}-06$ & $4.16 \mathrm{E}-06$ & $3.38 \mathrm{E}-06$ & $9.47 \mathrm{E}-07$ & $7.21 \mathrm{E}-09$ & $1.24 \mathrm{E}-11$ & $2.14 \mathrm{E}-14$ & $3.90 \mathrm{E}-17$ & 1.67E-24 & $0.00 \mathrm{E}+00$ \\
\hline ZR 93 & $2.53 \mathrm{E}-10$ & $2.53 \mathrm{E}-10$ & $2.53 \mathrm{E}-10$ & $2.53 \mathrm{E}-10$ & $2.53 \mathrm{E}-10$ & $2.53 \mathrm{E}-10$ & $2.53 \mathrm{E}-10$ & $2.53 \mathrm{E}-10$ & $2.53 \mathrm{E}-10$ & $2.53 \mathrm{E}-10$ \\
\hline ZR 95 & $1.31 \mathrm{E}-05$ & $1.31 \mathrm{E}-05$ & $1.29 \mathrm{E}-05$ & $1.21 \mathrm{E}-05$ & 9.45E-06 & $6.83 \mathrm{E}-06$ & $4.94 \mathrm{E}-06$ & $3.57 \mathrm{E}-06$ & 1.50E-06 & $2.65 \mathrm{E}-07$ \\
\hline ZR 97 & $3.30 \mathrm{E}-07$ & $3.24 \mathrm{E}-07$ & $1.23 \mathrm{E}-07$ & $3.37 \mathrm{E}-10$ & $4.95 \mathrm{E}-20$ & $0.00 \mathrm{E}+00$ & $0.00 \mathrm{E}+00$ & $0.00 \mathrm{E}+00$ & $0.00 \mathrm{E}+00$ & $0.00 \mathrm{E}+00$ \\
\hline NB 92 & $8.31 \mathrm{E}-04$ & $8.30 \mathrm{E}-04$ & $7.77 \mathrm{E}-04$ & $5.16 \mathrm{E}-04$ & 1.07E-04 & $1.39 \mathrm{E}-05$ & $1.79 \mathrm{E}-06$ & $2.31 \mathrm{E}-07$ & $9.86 \mathrm{E}-10$ & 1.79E-14 \\
\hline NB 93M & $3.64 \mathrm{E}-12$ & $3.64 \mathrm{E}-12$ & $3.68 \mathrm{E}-12$ & $3.87 \mathrm{E}-12$ & $4.63 \mathrm{E}-12$ & $5.61 \mathrm{E}-12$ & $6.59 \mathrm{E}-12$ & $7.57 \mathrm{E}-12$ & $1.01 \mathrm{E}-11$ & $1.52 \mathrm{E}-11$ \\
\hline NB 94 & $4.18 \mathrm{E}-05$ & $4.18 \mathrm{E}-05$ & $4.18 \mathrm{E}-05$ & $4.18 \mathrm{E}-05$ & $4.18 \mathrm{E}-05$ & $4.18 \mathrm{E}-05$ & $4.18 \mathrm{E}-05$ & 4.18E-05 & $4.18 \mathrm{E}-05$ & $4.18 \mathrm{E}-05$ \\
\hline NB 95 & $6.77 \mathrm{E}-02$ & $6.76 \mathrm{E}-02$ & $6.64 \mathrm{E}-02$ & $5.90 \mathrm{E}-02$ & $3.75 \mathrm{E}-02$ & $2.07 \mathrm{E}-02$ & $1.15 \mathrm{E}-02$ & $6.35 \mathrm{E}-03$ & $1.31 \mathrm{E}-03$ & $5.65 \mathrm{E}-05$ \\
\hline NB 95M & $9.10 \mathrm{E}-08$ & $9.10 \mathrm{E}-08$ & $9.10 \mathrm{E}-08$ & $8.84 \mathrm{E}-08$ & $7.01 \mathrm{E}-08$ & 5.07E-08 & $3.66 \mathrm{E}-08$ & $2.65 \mathrm{E}-08$ & $1.11 \mathrm{E}-08$ & 1.97E-09 \\
\hline NB 96 & $1.62 \mathrm{E}-04$ & $1.60 \mathrm{E}-04$ & $7.94 \mathrm{E}-05$ & $1.11 \mathrm{E}-06$ & $8.46 \mathrm{E}-14$ & $4.41 \mathrm{E}-23$ & $0.00 \mathrm{E}+00$ & $0.00 \mathrm{E}+00$ & $0.00 \mathrm{E}+00$ & $0.00 \mathrm{E}+00$ \\
\hline NB 97 & $1.17 E-05$ & $8.86 \mathrm{E}-06$ & $1.24 \mathrm{E}-07$ & $3.38 \mathrm{E}-10$ & $5.33 \mathrm{E}-20$ & $0.00 E+00$ & $0.00 \mathrm{E}+00$ & $0.00 \mathrm{E}+00$ & $0.00 \mathrm{E}+00$ & $0.00 \mathrm{E}+00$ \\
\hline NB 97M & $3.13 E-07$ & $3.06 \mathrm{E}-07$ & 1.17E-07 & $3.19 \mathrm{E}-10$ & $4.69 \mathrm{E}-20$ & $0.00 \mathrm{E}+00$ & $0.00 \mathrm{E}+00$ & $0.00 \mathrm{E}+00$ & $0.00 \mathrm{E}+00$ & $0.00 \mathrm{E}+00$ \\
\hline NB 98 & $1.75 \mathrm{E}-06$ & $0.00 \mathrm{E}+00$ & $0.00 \mathrm{E}+00$ & $0.00 \mathrm{E}+00$ & $0.00 \mathrm{E}+00$ & $0.00 \mathrm{E}+00$ & $0.00 \mathrm{E}+00$ & $0.00 \mathrm{E}+00$ & $0.00 \mathrm{E}+00$ & $0.00 \mathrm{E}+00$ \\
\hline NB100 & $8.67 E-08$ & $0.00 \mathrm{E}+00$ & $0.00 \mathrm{E}+00$ & $0.00 \mathrm{E}+00$ & $0.00 \mathrm{E}+00$ & $0.00 \mathrm{E}+00$ & $0.00 \mathrm{E}+00$ & $0.00 \mathrm{E}+00$ & $0.00 \mathrm{E}+00$ & $0.00 \mathrm{E}+00$ \\
\hline MO 93M & $2.41 \mathrm{E}-04$ & $2.30 \mathrm{E}-04$ & $2.13 \mathrm{E}-05$ & $1.00 \mathrm{E}-11$ & $0.00 \mathrm{E}+00$ & $0.00 \mathrm{E}+00$ & $0.00 \mathrm{E}+00$ & $0.00 \mathrm{E}+00$ & $0.00 \mathrm{E}+00$ & $0.00 \mathrm{E}+00$ \\
\hline MO 93 & $8.84 \mathrm{E}-07$ & $8.84 \mathrm{E}-07$ & $8.84 \mathrm{E}-07$ & $8.84 \mathrm{E}-07$ & $8.84 \mathrm{E}-07$ & 8.84E-07 & $8.84 \mathrm{E}-07$ & $8.84 \mathrm{E}-07$ & $8.84 \mathrm{E}-07$ & $8.84 \mathrm{E}-07$ \\
\hline MO 99 & $1.69 \mathrm{E}-01$ & $1.68 \mathrm{E}-01$ & $1.31 \mathrm{E}-01$ & $2.90 \mathrm{E}-02$ & $8.79 \mathrm{E}-05$ & 4.57E-08 & $2.38 \mathrm{E}-11$ & $1.24 \mathrm{E}-14$ & $2.16 \mathrm{E}-23$ & $0.00 \mathrm{E}+00$ \\
\hline MO101 & $4.20 \mathrm{E}-02$ & $1.01 \mathrm{E}-02$ & $0.00 \mathrm{E}+00$ & $0.00 \mathrm{E}+00$ & $0.00 \mathrm{E}+00$ & $0.00 E+00$ & $0.00 \mathrm{E}+00$ & $0.00 \mathrm{E}+00$ & $0.00 \mathrm{E}+00$ & $0.00 \mathrm{E}+00$ \\
\hline TC 99 & $3.86 \mathrm{E}-08$ & $3.86 \mathrm{E}-08$ & $3.88 \mathrm{E}-08$ & $3.92 \mathrm{E}-08$ & $3.94 \mathrm{E}-08$ & $3.94 \mathrm{E}-08$ & $3.94 \mathrm{E}-08$ & $3.94 \mathrm{E}-08$ & $3.94 \mathrm{E}-08$ & $3.94 \mathrm{E}-08$ \\
\hline TC100 & $81 \mathrm{E}-03$ & $0.00 \mathrm{E}+00$ & 0.00 & 0.00 & 00 & 00 & 00 & $0.00 \mathrm{E}+00$ & $E+00$ & $.00 \mathrm{E}+00$ \\
\hline
\end{tabular}


TEM-10200-1

12/19/17

Rev.08

Title: AS-RUN NEUTRONICS EVALUATION FOR THE GE HITACHI EXPERIMENT IN THE ATR

ECAR NO.

\begin{tabular}{|c|c|c|c|c|c|c|c|c|c|c|}
\hline \multicolumn{11}{|c|}{$1 \mathrm{~g}$ of Inconel } \\
\hline & EOI & $30.0 \mathrm{MI}$ & $1.0 \mathrm{D}$ & $7.0 \mathrm{D}$ & $30.0 \mathrm{D}$ & $60.0 \mathrm{D}$ & $90.0 \mathrm{D}$ & $120.0 \mathrm{D}$ & $200.0 \mathrm{D}$ & $360.0 \mathrm{D}$ \\
\hline Isotope & $\mathrm{Ci} / 1 \mathrm{~g}$ of incon & $\mathrm{Ci} / 1 \mathrm{~g}$ of incon & $\mathrm{Ci} / 1 \mathrm{~g}$ of incon & $\mathrm{Ci} / 1 \mathrm{~g}$ of incon & $\mathrm{Ci} / 1 \mathrm{~g}$ of incon & $\mathrm{Ci} / 1 \mathrm{~g}$ of incon & $\mathrm{Ci} / 1 \mathrm{~g}$ of incon & $\mathrm{Ci} / 1 \mathrm{~g}$ of incon & $\mathrm{Ci} / 1 \mathrm{~g}$ of incon & $\mathrm{Ci} / 1 \mathrm{~g}$ of incon \\
\hline TC101 & $4.20 \mathrm{E}-02$ & $2.43 \mathrm{E}-02$ & $0.00 \mathrm{E}+00$ & $0.00 \mathrm{E}+00$ & $0.00 \mathrm{E}+00$ & $0.00 \mathrm{E}+00$ & $0.00 \mathrm{E}+00$ & $0.00 \mathrm{E}+00$ & $0.00 \mathrm{E}+00$ & $0.00 \mathrm{E}+00$ \\
\hline RU103 & $9.89 \mathrm{E}-07$ & $9.89 \mathrm{E}-07$ & $9.72 \mathrm{E}-07$ & $8.74 \mathrm{E}-07$ & $5.83 \mathrm{E}-07$ & $3.43 \mathrm{E}-07$ & $2.02 \mathrm{E}-07$ & $1.19 \mathrm{E}-07$ & $2.90 \mathrm{E}-08$ & $1.72 \mathrm{E}-09$ \\
\hline RU105 & $5.13 \mathrm{E}-12$ & $4.75 \mathrm{E}-12$ & $1.21 \mathrm{E}-13$ & $2.08 \mathrm{E}-23$ & $0.00 E+00$ & $0.00 \mathrm{E}+00$ & $0.00 \mathrm{E}+00$ & $0.00 \mathrm{E}+00$ & $0.00 \mathrm{E}+00$ & $0.00 \mathrm{E}+00$ \\
\hline RH104 & $1.02 \mathrm{E}-08$ & $7.34 \mathrm{E}-12$ & $0.00 \mathrm{E}+00$ & $0.00 \mathrm{E}+00$ & $0.00 \mathrm{E}+00$ & $0.00 \mathrm{E}+00$ & $0.00 \mathrm{E}+00$ & $0.00 \mathrm{E}+00$ & $0.00 \mathrm{E}+00$ & $0.00 \mathrm{E}+00$ \\
\hline RH104M & $7.42 \mathrm{E}-10$ & $6.16 \mathrm{E}-12$ & $0.00 \mathrm{E}+00$ & $0.00 \mathrm{E}+00$ & $0.00 \mathrm{E}+00$ & $0.00 \mathrm{E}+00$ & $0.00 \mathrm{E}+00$ & $0.00 \mathrm{E}+00$ & $0.00 \mathrm{E}+00$ & $0.00 \mathrm{E}+00$ \\
\hline RH105 & $4.20 \mathrm{E}-12$ & $4.21 \mathrm{E}-12$ & $3.07 E-12$ & $1.83 \mathrm{E}-13$ & 3.67E-18 & $2.72 \mathrm{E}-24$ & $2.02 \mathrm{E}-30$ & $0.00 \mathrm{E}+00$ & $0.00 \mathrm{E}+00$ & $0.00 \mathrm{E}+00$ \\
\hline RH105M & 1.44E-12 & $1.33 \mathrm{E}-12$ & $3.40 \mathrm{E}-14$ & $5.83 \mathrm{E}-24$ & $0.00 \mathrm{E}+00$ & $0.00 \mathrm{E}+00$ & $0.00 \mathrm{E}+00$ & $0.00 \mathrm{E}+00$ & $0.00 \mathrm{E}+00$ & $0.00 \mathrm{E}+00$ \\
\hline RH106 & $4.15 \mathrm{E}-13$ & $1.41 \mathrm{E}-18$ & $1.41 \mathrm{E}-18$ & $1.41 \mathrm{E}-18$ & $1.35 \mathrm{E}-18$ & $1.27 \mathrm{E}-18$ & $1.20 \mathrm{E}-18$ & $1.13 \mathrm{E}-18$ & $9.74 \mathrm{E}-19$ & $7.16 \mathrm{E}-19$ \\
\hline RH106M & $2.02 \mathrm{E}-13$ & $1.73 \mathrm{E}-13$ & 1.05E-16 & $0.00 \mathrm{E}+00$ & $0.00 \mathrm{E}+00$ & $0.00 \mathrm{E}+00$ & $0.00 \mathrm{E}+00$ & $0.00 \mathrm{E}+00$ & $0.00 \mathrm{E}+00$ & $0.00 \mathrm{E}+00$ \\
\hline TA182 & $6.20 \mathrm{E}-02$ & $6.20 \mathrm{E}-02$ & $6.16 \mathrm{E}-02$ & $5.94 \mathrm{E}-02$ & 5.17E-02 & $4.32 \mathrm{E}-02$ & $3.60 \mathrm{E}-02$ & $3.01 \mathrm{E}-02$ & $1.86 \mathrm{E}-02$ & $7.08 \mathrm{E}-03$ \\
\hline TA182M & $1.84 \mathrm{E}-04$ & $5.22 \mathrm{E}-05$ & $0.00 \mathrm{E}+00$ & $0.00 \mathrm{E}+00$ & $0.00 \mathrm{E}+00$ & $0.00 \mathrm{E}+00$ & $0.00 \mathrm{E}+00$ & $0.00 \mathrm{E}+00$ & $0.00 \mathrm{E}+00$ & $0.00 \mathrm{E}+00$ \\
\hline TA183 & $3.25 \mathrm{E}-01$ & $3.24 \mathrm{E}-01$ & $2.84 \mathrm{E}-01$ & $1.26 \mathrm{E}-01$ & $5.51 \mathrm{E}-03$ & $9.34 \mathrm{E}-05$ & $1.58 \mathrm{E}-06$ & $2.68 \mathrm{E}-08$ & $5.09 \mathrm{E}-13$ & $1.83 \mathrm{E}-22$ \\
\hline W183M & $2.52 \mathrm{E}-05$ & $0.00 \mathrm{E}+00$ & $0.00 \mathrm{E}+00$ & $0.00 \mathrm{E}+00$ & $0.00 \mathrm{E}+00$ & $0.00 \mathrm{E}+00$ & $0.00 \mathrm{E}+00$ & $0.00 \mathrm{E}+00$ & $0.00 \mathrm{E}+00$ & $0.00 \mathrm{E}+00$ \\
\hline W185 & $2.40 \mathrm{E}-05$ & $2.40 \mathrm{E}-05$ & $2.38 \mathrm{E}-05$ & $2.25 \mathrm{E}-05$ & $1.82 \mathrm{E}-05$ & $1.38 \mathrm{E}-05$ & 1.05E-05 & 7.93E-06 & $3.79 \mathrm{E}-06$ & $8.65 \mathrm{E}-07$ \\
\hline W185M & $6.52 \mathrm{E}-08$ & $2.55 \mathrm{E}-13$ & $0.00 \mathrm{E}+00$ & $0.00 \mathrm{E}+00$ & $0.00 \mathrm{E}+00$ & $0.00 \mathrm{E}+00$ & $0.00 \mathrm{E}+00$ & $0.00 \mathrm{E}+00$ & $0.00 \mathrm{E}+00$ & $0.00 \mathrm{E}+00$ \\
\hline W187 & $4.35 \mathrm{E}-09$ & $4.28 \mathrm{E}-09$ & 2.17E-09 & $3.33 \mathrm{E}-11$ & $3.71 \mathrm{E}-18$ & $3.17 E-27$ & $0.00 \mathrm{E}+00$ & $0.00 \mathrm{E}+00$ & $0.00 \mathrm{E}+00$ & $0.00 \mathrm{E}+00$ \\
\hline W188 & $5.50 \mathrm{E}-12$ & $5.50 \mathrm{E}-12$ & $5.45 \mathrm{E}-12$ & $5.13 E-12$ & $4.08 \mathrm{E}-12$ & $3.02 \mathrm{E}-12$ & $2.24 \mathrm{E}-12$ & $1.66 \mathrm{E}-12$ & $7.47 \mathrm{E}-13$ & $1.51 \mathrm{E}-13$ \\
\hline RE186 & $2.47 \mathrm{E}-06$ & $2.46 \mathrm{E}-06$ & $2.05 \mathrm{E}-06$ & $6.82 \mathrm{E}-07$ & $1.00 \mathrm{E}-08$ & 4.07E-11 & $1.65 \mathrm{E}-13$ & $6.69 \mathrm{E}-16$ & $2.81 \mathrm{E}-22$ & $0.00 \mathrm{E}+00$ \\
\hline RE188 & $9.23 \mathrm{E}-11$ & $9.17 \mathrm{E}-11$ & 3.87E-11 & $5.28 \mathrm{E}-12$ & $4.12 \mathrm{E}-12$ & $3.05 \mathrm{E}-12$ & $2.26 \mathrm{E}-12$ & $1.68 \mathrm{E}-12$ & $7.54 \mathrm{E}-13$ & $1.53 \mathrm{E}-13$ \\
\hline RE188M & $8.79 \mathrm{E}-11$ & $2.89 \mathrm{E}-11$ & $0.00 \mathrm{E}+00$ & $0.00 \mathrm{E}+00$ & $0.00 \mathrm{E}+00$ & $0.00 \mathrm{E}+00$ & $0.00 \mathrm{E}+00$ & $0.00 \mathrm{E}+00$ & $0.00 \mathrm{E}+00$ & $0.00 \mathrm{E}+00$ \\
\hline SUMTOT & $8.97 E+00$ & $5.48 \mathrm{E}+00$ & $3.77 E+00$ & $3.14 \mathrm{E}+00$ & $2.06 \mathrm{E}+00$ & $1.39 \mathrm{E}+00$ & $1.04 \mathrm{E}+00$ & $8.52 \mathrm{E}-01$ & $6.46 \mathrm{E}-01$ & $5.35 \mathrm{E}-01$ \\
\hline OTOTAL & $8.97 \mathrm{E}+00$ & $5.48 \mathrm{E}+00$ & $3.77 \mathrm{E}+00$ & $3.14 \mathrm{E}+00$ & $2.06 \mathrm{E}+00$ & $1.39 \mathrm{E}+00$ & $1.04 \mathrm{E}+00$ & $8.52 \mathrm{E}-01$ & $6.46 \mathrm{E}-01$ & $5.35 \mathrm{E}-01$ \\
\hline
\end{tabular}

Table 18. $\mathrm{g} / \mathrm{g}$ of incon

\begin{tabular}{|c|c|c|c|c|c|c|c|c|c|c|}
\hline \multicolumn{11}{|c|}{$1 \mathrm{~g}$ of Inconel } \\
\hline Isctono & EOI & $30.0 \mathrm{MI}$ & $1.0 \mathrm{D}$ & $7.0 \mathrm{D}$ & $30.0 \mathrm{D}$ & $60.0 \mathrm{D}$ & $90.0 \mathrm{D}$ & $120.0 \mathrm{D}$ & $200.0 \mathrm{D}$ & $360.0 \mathrm{D}$ \\
\hline Isotope & $\mathrm{g} / 1 \mathrm{~g}$ of incon & $\mathrm{g} / 1 \mathrm{~g}$ of incon & $\mathrm{g} / 1 \mathrm{~g}$ of incon & $\mathrm{g} / 1 \mathrm{~g}$ of incon & $\mathrm{g} / 1 \mathrm{~g}$ of incon & $\mathrm{g} / 1 \mathrm{~g}$ of incon & $\mathrm{g} / 1 \mathrm{~g}$ of incon & $\mathrm{g} / 1 \mathrm{~g}$ of incon & $\mathrm{g} / 1 \mathrm{~g}$ of incon & $\mathrm{g} / 1 \mathrm{~g}$ of incon \\
\hline H 1 & $1.45 \mathrm{E}-06$ & 1.45E-06 & $1.45 \mathrm{E}-06$ & 1.45E-06 & $1.45 \mathrm{E}-06$ & $1.45 \mathrm{E}-06$ & 1.45E-06 & $1.45 \mathrm{E}-06$ & 1.45E-06 & 1.45E-06 \\
\hline H 2 & $5.05 \mathrm{E}-10$ & $5.05 \mathrm{E}-10$ & $5.05 \mathrm{E}-10$ & $5.05 \mathrm{E}-10$ & $5.05 \mathrm{E}-10$ & $5.05 \mathrm{E}-10$ & $5.05 \mathrm{E}-10$ & $5.05 \mathrm{E}-10$ & $5.05 \mathrm{E}-10$ & $5.05 \mathrm{E}-10$ \\
\hline H 3 & $3.36 \mathrm{E}-14$ & $3.36 \mathrm{E}-14$ & $3.36 \mathrm{E}-14$ & $3.35 \mathrm{E}-14$ & $3.34 \mathrm{E}-14$ & $3.33 \mathrm{E}-14$ & $3.31 \mathrm{E}-14$ & $3.30 \mathrm{E}-14$ & $3.26 \mathrm{E}-14$ & $3.18 \mathrm{E}-14$ \\
\hline HE 3 & $1.30 \mathrm{E}-16$ & $1.30 \mathrm{E}-16$ & $1.35 \mathrm{E}-16$ & $1.66 \mathrm{E}-16$ & $2.84 \mathrm{E}-16$ & $4.38 \mathrm{E}-16$ & $5.91 \mathrm{E}-16$ & 7.43E-16 & $1.15 \mathrm{E}-15$ & $1.94 \mathrm{E}-15$ \\
\hline HE 4 & 5.96E-06 & $5.96 \mathrm{E}-06$ & $5.96 \mathrm{E}-06$ & $5.96 \mathrm{E}-06$ & $5.96 \mathrm{E}-06$ & $5.96 \mathrm{E}-06$ & $5.96 \mathrm{E}-06$ & $5.96 \mathrm{E}-06$ & $5.96 \mathrm{E}-06$ & 5.96E-06 \\
\hline LI 6 & $2.28 \mathrm{E}-13$ & $2.28 \mathrm{E}-13$ & $2.28 \mathrm{E}-13$ & $2.28 \mathrm{E}-13$ & $2.28 \mathrm{E}-13$ & $2.28 \mathrm{E}-13$ & $2.28 \mathrm{E}-13$ & $2.28 \mathrm{E}-13$ & $2.28 \mathrm{E}-13$ & $2.28 \mathrm{E}-13$ \\
\hline LI 7 & $7.64 \mathrm{E}-06$ & $7.64 \mathrm{E}-06$ & $7.64 \mathrm{E}-06$ & $7.64 \mathrm{E}-06$ & $7.64 \mathrm{E}-06$ & $7.64 \mathrm{E}-06$ & $7.64 \mathrm{E}-06$ & $7.64 \mathrm{E}-06$ & $7.64 \mathrm{E}-06$ & $7.64 \mathrm{E}-06$ \\
\hline
\end{tabular}


TEM-10200-1

$12 / 19 / 17$

Rev.08

Title: AS-RUN NEUTRONICS EVALUATION FOR THE GE HITACHI EXPERIMENT IN THE ATR ECAR NO.: $4740 \quad$ REV. NO.: 0

\begin{tabular}{|c|c|c|c|c|c|c|c|c|c|c|}
\hline \multicolumn{11}{|c|}{$1 \mathrm{~g}$ of Inconel } \\
\hline Isotone & EOI & $30.0 \mathrm{MI}$ & $1.0 \mathrm{D}$ & $7.0 \mathrm{D}$ & $30.0 \mathrm{D}$ & $60.0 \mathrm{D}$ & $90.0 \mathrm{D}$ & $120.0 \mathrm{D}$ & $200.0 \mathrm{D}$ & $360.0 \mathrm{D}$ \\
\hline (150tupe & $\mathrm{g} / 1 \mathrm{~g}$ of incon & $\mathrm{g} / 1 \mathrm{~g}$ of incon & $\mathrm{g} / 1 \mathrm{~g}$ of incon & $\mathrm{g} / 1 \mathrm{~g}$ of incon & $\mathrm{g} / \mathrm{g}$ of incon & $\mathrm{g} / 1 \mathrm{~g}$ of incon & $\mathrm{g} / 1 \mathrm{~g}$ of incon & $\mathrm{g} / 1 \mathrm{~g}$ of incon & $\mathrm{g} / 1 \mathrm{~g}$ of incon & $\mathrm{g} / 1 \mathrm{~g}$ of incon \\
\hline BE 9 & $2.31 \mathrm{E}-09$ & $2.31 \mathrm{E}-09$ & $2.31 \mathrm{E}-09$ & $2.31 \mathrm{E}-09$ & $2.31 \mathrm{E}-09$ & $2.31 \mathrm{E}-09$ & $2.31 \mathrm{E}-09$ & $2.31 \mathrm{E}-09$ & $2.31 \mathrm{E}-09$ & $2.31 \mathrm{E}-09$ \\
\hline BE 10 & $1.26 \mathrm{E}-10$ & $1.26 \mathrm{E}-10$ & $1.26 \mathrm{E}-10$ & $1.26 \mathrm{E}-10$ & $1.26 \mathrm{E}-10$ & $1.26 \mathrm{E}-10$ & $1.26 \mathrm{E}-10$ & $1.26 \mathrm{E}-10$ & $1.26 \mathrm{E}-10$ & $1.26 \mathrm{E}-10$ \\
\hline B 10 & 1.99E-07 & $1.99 \mathrm{E}-07$ & 1.99E-07 & $1.99 \mathrm{E}-07$ & $1.99 \mathrm{E}-07$ & $1.99 \mathrm{E}-07$ & 1.99E-07 & 1.99E-07 & $1.99 \mathrm{E}-07$ & $1.99 \mathrm{E}-07$ \\
\hline B 11 & 4.89E-05 & 4.89E-05 & 4.89E-05 & 4.89E-05 & 4.89E-05 & 4.89E-05 & 4.89E-05 & 4.89E-05 & 4.89E-05 & 4.89E-05 \\
\hline C 12 & $7.90 \mathrm{E}-04$ & $7.90 \mathrm{E}-04$ & $7.90 \mathrm{E}-04$ & $7.90 \mathrm{E}-04$ & $7.90 \mathrm{E}-04$ & $7.90 \mathrm{E}-04$ & $7.90 \mathrm{E}-04$ & $7.90 \mathrm{E}-04$ & $7.90 \mathrm{E}-04$ & $7.90 \mathrm{E}-04$ \\
\hline C 13 & $9.61 \mathrm{E}-06$ & $9.61 \mathrm{E}-06$ & $9.61 \mathrm{E}-06$ & $9.61 \mathrm{E}-06$ & $9.61 \mathrm{E}-06$ & $9.61 \mathrm{E}-06$ & $9.61 \mathrm{E}-06$ & $9.61 \mathrm{E}-06$ & $9.61 \mathrm{E}-06$ & $9.61 \mathrm{E}-06$ \\
\hline C 14 & $1.09 \mathrm{E}-11$ & 1.09E-11 & $1.09 \mathrm{E}-11$ & 1.09E-11 & $1.09 E-11$ & $1.09 \mathrm{E}-11$ & $1.09 \mathrm{E}-11$ & $1.09 E-11$ & $1.09 \mathrm{E}-11$ & $1.09 E-11$ \\
\hline N 14 & 3.95E-16 & $3.95 \mathrm{E}-16$ & $3.98 \mathrm{E}-16$ & 4.20E-16 & $5.03 E-16$ & $6.11 \mathrm{E}-16$ & 7.19E-16 & $8.27 \mathrm{E}-16$ & $1.11 \mathrm{E}-15$ & 1.69E-15 \\
\hline NE 21 & $2.84 \mathrm{E}-14$ & $2.84 \mathrm{E}-14$ & $2.84 \mathrm{E}-14$ & $2.84 \mathrm{E}-14$ & $2.84 \mathrm{E}-14$ & $2.84 \mathrm{E}-14$ & $2.84 \mathrm{E}-14$ & $2.84 \mathrm{E}-14$ & $2.84 \mathrm{E}-14$ & $2.84 \mathrm{E}-14$ \\
\hline NE 22 & $1.50 \mathrm{E}-13$ & $1.50 \mathrm{E}-13$ & $1.50 \mathrm{E}-13$ & $1.50 \mathrm{E}-13$ & $1.50 \mathrm{E}-13$ & $1.50 \mathrm{E}-13$ & $1.50 \mathrm{E}-13$ & $1.50 \mathrm{E}-13$ & $1.50 \mathrm{E}-13$ & $1.50 \mathrm{E}-13$ \\
\hline NA 23 & $1.21 \mathrm{E}-14$ & $1.21 \mathrm{E}-14$ & $1.21 \mathrm{E}-14$ & $1.21 \mathrm{E}-14$ & $1.21 \mathrm{E}-14$ & $1.21 \mathrm{E}-14$ & $1.21 \mathrm{E}-14$ & $1.21 \mathrm{E}-14$ & $1.21 \mathrm{E}-14$ & $1.21 \mathrm{E}-14$ \\
\hline NA 24 & $5.82 \mathrm{E}-11$ & 5.69E-11 & $1.92 \mathrm{E}-11$ & $2.47 \mathrm{E}-14$ & $2.06 \mathrm{E}-25$ & $0.00 \mathrm{E}+00$ & $0.00 \mathrm{E}+00$ & $0.00 \mathrm{E}+00$ & $0.00 \mathrm{E}+00$ & $0.00 \mathrm{E}+00$ \\
\hline MG 24 & $1.41 \mathrm{E}-08$ & $1.41 \mathrm{E}-08$ & $1.41 \mathrm{E}-08$ & $1.42 \mathrm{E}-08$ & $1.42 \mathrm{E}-08$ & $1.42 \mathrm{E}-08$ & $1.42 \mathrm{E}-08$ & $1.42 \mathrm{E}-08$ & $1.42 \mathrm{E}-08$ & $1.42 \mathrm{E}-08$ \\
\hline MG 25 & $6.52 \mathrm{E}-09$ & $6.52 \mathrm{E}-09$ & $6.52 \mathrm{E}-09$ & $6.52 \mathrm{E}-09$ & $6.52 \mathrm{E}-09$ & $6.52 \mathrm{E}-09$ & $6.52 \mathrm{E}-09$ & $6.52 \mathrm{E}-09$ & $6.52 \mathrm{E}-09$ & $6.52 \mathrm{E}-09$ \\
\hline MG 26 & 2.97E-09 & 2.97E-09 & 2.97E-09 & 2.97E-09 & 2.97E-09 & 2.97E-09 & 2.97E-09 & 2.97E-09 & 2.97E-09 & $2.97 \mathrm{E}-09$ \\
\hline MG 27 & 3.29E-12 & 3.66E-13 & $0.00 \mathrm{E}+00$ & $0.00 \mathrm{E}+00$ & $0.00 \mathrm{E}+00$ & $0.00 \mathrm{E}+00$ & $0.00 \mathrm{E}+00$ & $0.00 \mathrm{E}+00$ & $0.00 \mathrm{E}+00$ & $0.00 \mathrm{E}+00$ \\
\hline AL 27 & $8.00 \mathrm{E}-03$ & 8.00E-03 & $8.00 \mathrm{E}-03$ & 8.00E-03 & $8.00 \mathrm{E}-03$ & $8.00 \mathrm{E}-03$ & $8.00 \mathrm{E}-03$ & $8.00 \mathrm{E}-03$ & 8.00E-03 & $8.00 \mathrm{E}-03$ \\
\hline AL 28 & $1.98 \mathrm{E}-11$ & $1.84 \mathrm{E}-15$ & $5.81 \mathrm{E}-22$ & 4.91E-24 & $5.56 \mathrm{E}-32$ & $0.00 \mathrm{E}+00$ & $0.00 \mathrm{E}+00$ & $0.00 \mathrm{E}+00$ & $0.00 \mathrm{E}+00$ & $0.00 \mathrm{E}+00$ \\
\hline AL 29 & $4.18 \mathrm{E}-14$ & $1.72 \mathrm{E}-15$ & $0.00 \mathrm{E}+00$ & $0.00 \mathrm{E}+00$ & $0.00 \mathrm{E}+00$ & $0.00 \mathrm{E}+00$ & $0.00 \mathrm{E}+00$ & $0.00 \mathrm{E}+00$ & $0.00 \mathrm{E}+00$ & $0.00 \mathrm{E}+00$ \\
\hline SI 28 & $3.22 \mathrm{E}-03$ & $3.22 \mathrm{E}-03$ & $3.22 \mathrm{E}-03$ & $3.22 \mathrm{E}-03$ & $3.22 \mathrm{E}-03$ & $3.22 \mathrm{E}-03$ & $3.22 \mathrm{E}-03$ & $3.22 \mathrm{E}-03$ & $3.22 \mathrm{E}-03$ & $3.22 \mathrm{E}-03$ \\
\hline SI 29 & $1.69 \mathrm{E}-04$ & 1.69E-04 & $1.69 \mathrm{E}-04$ & 1.69E-04 & $1.69 \mathrm{E}-04$ & $1.69 \mathrm{E}-04$ & 1.69E-04 & $1.69 \mathrm{E}-04$ & $1.69 \mathrm{E}-04$ & $1.69 \mathrm{E}-04$ \\
\hline SI 30 & $1.16 \mathrm{E}-04$ & 1.16E-04 & $1.16 \mathrm{E}-04$ & $1.16 \mathrm{E}-04$ & $1.16 \mathrm{E}-04$ & $1.16 \mathrm{E}-04$ & $1.16 \mathrm{E}-04$ & $1.16 \mathrm{E}-04$ & $1.16 \mathrm{E}-04$ & $1.16 \mathrm{E}-04$ \\
\hline SI 31 & 2.00E-11 & 1.75E-11 & $3.51 \mathrm{E}-14$ & $1.02 \mathrm{E}-30$ & $0.00 \mathrm{E}+00$ & $0.00 \mathrm{E}+00$ & $0.00 \mathrm{E}+00$ & $0.00 \mathrm{E}+00$ & $0.00 \mathrm{E}+00$ & $0.00 \mathrm{E}+00$ \\
\hline SI 32 & $9.11 \mathrm{E}-15$ & $9.11 \mathrm{E}-15$ & $9.11 \mathrm{E}-15$ & $9.11 \mathrm{E}-15$ & $9.11 \mathrm{E}-15$ & $9.11 \mathrm{E}-15$ & $9.11 \mathrm{E}-15$ & $9.11 \mathrm{E}-15$ & $9.11 \mathrm{E}-15$ & $9.11 \mathrm{E}-15$ \\
\hline P 31 & $1.50 \mathrm{E}-04$ & $1.50 \mathrm{E}-04$ & $1.50 \mathrm{E}-04$ & $1.50 \mathrm{E}-04$ & $1.50 \mathrm{E}-04$ & $1.50 \mathrm{E}-04$ & $1.50 \mathrm{E}-04$ & $1.50 \mathrm{E}-04$ & 1.50E-04 & $1.50 \mathrm{E}-04$ \\
\hline P 32 & 4.87E-09 & 4.87E-09 & 4.64E-09 & 3.47E-09 & 1.14E-09 & $2.66 \mathrm{E}-10$ & $6.21 \mathrm{E}-11$ & $1.45 \mathrm{E}-11$ & $3.00 \mathrm{E}-13$ & $1.29 \mathrm{E}-16$ \\
\hline P 33 & $3.68 \mathrm{E}-13$ & 3.67E-13 & $3.58 \mathrm{E}-13$ & 3.03E-13 & $1.60 \mathrm{E}-13$ & $6.96 \mathrm{E}-14$ & 3.03E-14 & 1.32E-14 & $1.44 \mathrm{E}-15$ & $1.70 \mathrm{E}-17$ \\
\hline S 32 & 1.42E-04 & $1.42 \mathrm{E}-04$ & $1.42 \mathrm{E}-04$ & $1.42 \mathrm{E}-04$ & 1.42E-04 & 1.42E-04 & $1.42 \mathrm{E}-04$ & 1.42E-04 & $1.42 \mathrm{E}-04$ & $1.42 \mathrm{E}-04$ \\
\hline S 33 & $1.23 \mathrm{E}-06$ & $1.23 \mathrm{E}-06$ & $1.23 \mathrm{E}-06$ & $1.23 \mathrm{E}-06$ & $1.23 \mathrm{E}-06$ & $1.23 \mathrm{E}-06$ & $1.23 \mathrm{E}-06$ & 1.23E-06 & $1.23 \mathrm{E}-06$ & $1.23 \mathrm{E}-06$ \\
\hline S 34 & $6.69 \mathrm{E}-06$ & 6.69E-06 & $6.69 \mathrm{E}-06$ & 6.69E-06 & $6.69 \mathrm{E}-06$ & $6.69 \mathrm{E}-06$ & $6.69 \mathrm{E}-06$ & $6.69 \mathrm{E}-06$ & $6.69 \mathrm{E}-06$ & $6.69 \mathrm{E}-06$ \\
\hline S 35 & $7.25 \mathrm{E}-10$ & $7.25 \mathrm{E}-10$ & 7.19E-10 & $6.86 \mathrm{E}-10$ & $5.72 \mathrm{E}-10$ & 4.52E-10 & 3.57E-10 & 2.82E-10 & $1.50 \mathrm{E}-10$ & 4.26E-11 \\
\hline S 36 & $2.86 \mathrm{E}-08$ & $2.86 \mathrm{E}-08$ & $2.86 \mathrm{E}-08$ & $2.86 \mathrm{E}-08$ & $2.86 \mathrm{E}-08$ & $2.86 \mathrm{E}-08$ & $2.86 \mathrm{E}-08$ & $2.86 \mathrm{E}-08$ & $2.86 \mathrm{E}-08$ & $2.86 \mathrm{E}-08$ \\
\hline CL 35 & 7.87E-10 & 7.87E-10 & 7.93E-10 & $8.26 \mathrm{E}-10$ & 9.39E-10 & $1.06 \mathrm{E}-09$ & 1.16E-09 & $1.23 \mathrm{E}-09$ & $1.36 \mathrm{E}-09$ & $1.47 \mathrm{E}-09$ \\
\hline CL 36 & $1.19 \mathrm{E}-11$ & $1.19 \mathrm{E}-11$ & $1.19 \mathrm{E}-11$ & $1.19 \mathrm{E}-11$ & $1.19 \mathrm{E}-11$ & $1.19 \mathrm{E}-11$ & $1.19 \mathrm{E}-11$ & $1.19 \mathrm{E}-11$ & $1.19 \mathrm{E}-11$ & $1.19 \mathrm{E}-11$ \\
\hline CL 37 & 4.09E-12 & 4.09E-12 & 4.09E-12 & $4.09 \mathrm{E}-12$ & 4.09E-12 & 4.09E-12 & 4.09E-12 & 4.09E-12 & 4.09E-12 & 4.09E-12 \\
\hline AR 40 & 2.07E-14 & 2.07E-14 & 2.07E-14 & 2.07E-14 & 2.07E-14 & 2.07E-14 & 2.07E-14 & 2.07E-14 & 2.07E-14 & 2.07E-14 \\
\hline CA 43 & $1.72 \mathrm{E}-09$ & $1.72 \mathrm{E}-09$ & $1.72 \mathrm{E}-09$ & $1.72 \mathrm{E}-09$ & $1.72 \mathrm{E}-09$ & $1.72 \mathrm{E}-09$ & $1.72 \mathrm{E}-09$ & $1.72 \mathrm{E}-09$ & $1.72 \mathrm{E}-09$ & $1.72 \mathrm{E}-09$ \\
\hline
\end{tabular}


TEM-10200-1

12/19/17

Rev.08

Title: AS-RUN NEUTRONICS EVALUATION FOR THE GE HITACHI EXPERIMENT IN THE ATR ECAR NO.: 4740 REV. NO.: 0

\begin{tabular}{|c|c|c|c|c|c|c|c|c|c|c|}
\hline \multicolumn{11}{|c|}{$1 \mathrm{~g}$ of Inconel } \\
\hline & EOI & $30.0 \mathrm{MI}$ & $1.0 \mathrm{D}$ & $7.0 \mathrm{D}$ & $30.0 \mathrm{D}$ & $60.0 \mathrm{D}$ & $90.0 \mathrm{D}$ & $120.0 \mathrm{D}$ & $200.0 \mathrm{D}$ & $360.0 \mathrm{D}$ \\
\hline Isotope & $\mathrm{g} / 1 \mathrm{~g}$ of incon & $\mathrm{g} / 1 \mathrm{~g}$ of incon & $\mathrm{g} / 1 \mathrm{~g}$ of incon & $\mathrm{g} / 1 \mathrm{~g}$ of incon & $\mathrm{g} / 1 \mathrm{~g}$ of incon & $\mathrm{g} / 1 \mathrm{~g}$ of incon & $\mathrm{g} / 1 \mathrm{~g}$ of incon & $\mathrm{g} / 1 \mathrm{~g}$ of incon & $\mathrm{g} / 1 \mathrm{~g}$ of incon & $\mathrm{g} / 1 \mathrm{~g}$ of incon \\
\hline CA 44 & $1.33 \mathrm{E}-08$ & $1.33 \mathrm{E}-08$ & $1.33 \mathrm{E}-08$ & $1.33 \mathrm{E}-08$ & $1.33 \mathrm{E}-08$ & $1.33 \mathrm{E}-08$ & $1.33 \mathrm{E}-08$ & $1.33 \mathrm{E}-08$ & $1.33 \mathrm{E}-08$ & $1.33 \mathrm{E}-08$ \\
\hline CA 45 & $1.80 \mathrm{E}-10$ & $1.80 \mathrm{E}-10$ & $1.79 \mathrm{E}-10$ & $1.74 \mathrm{E}-10$ & $1.58 \mathrm{E}-10$ & $1.39 \mathrm{E}-10$ & $1.23 \mathrm{E}-10$ & $1.08 \mathrm{E}-10$ & 7.67E-11 & $3.89 \mathrm{E}-11$ \\
\hline CA 46 & $1.55 \mathrm{E}-09$ & $1.55 \mathrm{E}-09$ & $1.55 \mathrm{E}-09$ & $1.55 \mathrm{E}-09$ & $1.55 \mathrm{E}-09$ & $1.55 \mathrm{E}-09$ & $1.55 \mathrm{E}-09$ & $1.55 \mathrm{E}-09$ & $1.55 \mathrm{E}-09$ & $1.55 \mathrm{E}-09$ \\
\hline CA 47 & $5.13 \mathrm{E}-14$ & $5.11 \mathrm{E}-14$ & 4.40E-14 & $1.76 \mathrm{E}-14$ & $5.24 \mathrm{E}-16$ & 5.35E-18 & $5.46 \mathrm{E}-20$ & $5.59 \mathrm{E}-22$ & $2.74 \mathrm{E}-27$ & $0.00 \mathrm{E}+00$ \\
\hline SC 45 & $9.50 \mathrm{E}-11$ & $9.50 \mathrm{E}-11$ & $9.58 \mathrm{E}-11$ & $1.00 \mathrm{E}-10$ & 1.17E-10 & $1.36 \mathrm{E}-10$ & $1.52 \mathrm{E}-10$ & 1.67E-10 & $1.98 \mathrm{E}-10$ & $2.36 \mathrm{E}-10$ \\
\hline SC 46 & $1.65 \mathrm{E}-08$ & $1.65 \mathrm{E}-08$ & $1.64 \mathrm{E}-08$ & $1.56 \mathrm{E}-08$ & $1.29 \mathrm{E}-08$ & $1.01 \mathrm{E}-08$ & $7.84 \mathrm{E}-09$ & $6.12 \mathrm{E}-09$ & $3.16 \mathrm{E}-09$ & $8.40 \mathrm{E}-10$ \\
\hline SC 47 & $1.25 \mathrm{E}-09$ & $1.25 \mathrm{E}-09$ & $1.02 \mathrm{E}-09$ & $2.94 \mathrm{E}-10$ & $2.53 \mathrm{E}-12$ & $5.11 \mathrm{E}-15$ & 1.05E-17 & $2.24 \mathrm{E}-20$ & $9.10 \mathrm{E}-27$ & $0.00 \mathrm{E}+00$ \\
\hline SC 48 & $6.52 \mathrm{E}-11$ & $6.47 \mathrm{E}-11$ & $4.46 \mathrm{E}-11$ & $4.57 \mathrm{E}-12$ & $7.35 \mathrm{E}-16$ & $8.29 \mathrm{E}-21$ & $9.35 \mathrm{E}-26$ & $1.06 \mathrm{E}-30$ & $0.00 \mathrm{E}+00$ & $0.00 \mathrm{E}+00$ \\
\hline SC 49 & $1.29 \mathrm{E}-12$ & $8.99 \mathrm{E}-13$ & $3.73 \mathrm{E}-20$ & $0.00 \mathrm{E}+00$ & $0.00 \mathrm{E}+00$ & $0.00 \mathrm{E}+00$ & $0.00 \mathrm{E}+00$ & $0.00 \mathrm{E}+00$ & $0.00 \mathrm{E}+00$ & $0.00 \mathrm{E}+00$ \\
\hline TI 46 & $9.10 \mathrm{E}-04$ & $9.10 \mathrm{E}-04$ & $9.10 \mathrm{E}-04$ & $9.10 \mathrm{E}-04$ & $9.10 \mathrm{E}-04$ & $9.10 \mathrm{E}-04$ & $9.10 \mathrm{E}-04$ & $9.10 \mathrm{E}-04$ & $9.10 \mathrm{E}-04$ & $9.10 \mathrm{E}-04$ \\
\hline TI 47 & $8.39 \mathrm{E}-04$ & $8.39 \mathrm{E}-04$ & $8.39 \mathrm{E}-04$ & $8.39 \mathrm{E}-04$ & $8.39 \mathrm{E}-04$ & $8.39 \mathrm{E}-04$ & $8.39 \mathrm{E}-04$ & $8.39 \mathrm{E}-04$ & $8.39 \mathrm{E}-04$ & $8.39 \mathrm{E}-04$ \\
\hline TI 48 & $8.43 \mathrm{E}-03$ & $8.43 \mathrm{E}-03$ & $8.43 \mathrm{E}-03$ & $8.43 \mathrm{E}-03$ & $8.43 \mathrm{E}-03$ & $8.43 \mathrm{E}-03$ & $8.43 \mathrm{E}-03$ & $8.43 \mathrm{E}-03$ & $8.43 \mathrm{E}-03$ & $8.43 \mathrm{E}-03$ \\
\hline TI 49 & $6.96 \mathrm{E}-04$ & $6.96 \mathrm{E}-04$ & $6.96 \mathrm{E}-04$ & $6.96 \mathrm{E}-04$ & $6.96 \mathrm{E}-04$ & $6.96 \mathrm{E}-04$ & $6.96 \mathrm{E}-04$ & $6.96 \mathrm{E}-04$ & $6.96 \mathrm{E}-04$ & $6.96 \mathrm{E}-04$ \\
\hline TI 50 & $6.26 \mathrm{E}-04$ & $6.26 \mathrm{E}-04$ & $6.26 \mathrm{E}-04$ & $6.26 \mathrm{E}-04$ & $6.26 \mathrm{E}-04$ & $6.26 \mathrm{E}-04$ & $6.26 \mathrm{E}-04$ & $6.26 \mathrm{E}-04$ & $6.26 \mathrm{E}-04$ & $6.26 \mathrm{E}-04$ \\
\hline TI 51 & $2.94 \mathrm{E}-12$ & $7.96 E-14$ & $0.00 \mathrm{E}+00$ & $0.00 \mathrm{E}+00$ & $0.00 \mathrm{E}+00$ & $0.00 \mathrm{E}+00$ & $0.00 \mathrm{E}+00$ & $0.00 \mathrm{E}+00$ & $0.00 \mathrm{E}+00$ & $0.00 \mathrm{E}+00$ \\
\hline V 50 & $6.54 \mathrm{E}-07$ & $6.54 \mathrm{E}-07$ & $6.54 \mathrm{E}-07$ & $6.54 \mathrm{E}-07$ & $6.54 \mathrm{E}-07$ & $6.54 \mathrm{E}-07$ & $6.54 \mathrm{E}-07$ & $6.54 \mathrm{E}-07$ & $6.54 \mathrm{E}-07$ & $6.54 \mathrm{E}-07$ \\
\hline V 51 & 1.07E-04 & $1.07 E-04$ & $1.08 \mathrm{E}-04$ & 1.11E-04 & $1.20 \mathrm{E}-04$ & $1.26 \mathrm{E}-04$ & $1.29 \mathrm{E}-04$ & $1.30 \mathrm{E}-04$ & $1.31 \mathrm{E}-04$ & $1.31 \mathrm{E}-04$ \\
\hline V 52 & $2.28 \mathrm{E}-11$ & $8.92 \mathrm{E}-14$ & $0.00 \mathrm{E}+00$ & $0.00 \mathrm{E}+00$ & $0.00 \mathrm{E}+00$ & $0.00 E+00$ & $0.00 E+00$ & $0.00 \mathrm{E}+00$ & $0.00 \mathrm{E}+00$ & $0.00 E+00$ \\
\hline V 53 & $4.60 \mathrm{E}-14$ & $1.13 \mathrm{E}-19$ & $0.00 \mathrm{E}+00$ & $0.00 \mathrm{E}+00$ & $0.00 \mathrm{E}+00$ & $0.00 \mathrm{E}+00$ & $0.00 \mathrm{E}+00$ & $0.00 \mathrm{E}+00$ & $0.00 \mathrm{E}+00$ & $0.00 \mathrm{E}+00$ \\
\hline CR 50 & $8.65 \mathrm{E}-03$ & $8.65 \mathrm{E}-03$ & $8.65 \mathrm{E}-03$ & $8.65 \mathrm{E}-03$ & $8.65 \mathrm{E}-03$ & $8.65 \mathrm{E}-03$ & $8.65 \mathrm{E}-03$ & $8.65 \mathrm{E}-03$ & $8.65 \mathrm{E}-03$ & $8.65 \mathrm{E}-03$ \\
\hline CR 51 & $2.37 \mathrm{E}-05$ & $2.37 E-05$ & $2.31 \mathrm{E}-05$ & $1.99 \mathrm{E}-05$ & $1.12 \mathrm{E}-05$ & $5.28 \mathrm{E}-06$ & $2.49 \mathrm{E}-06$ & $1.18 \mathrm{E}-06$ & $1.59 \mathrm{E}-07$ & $2.91 \mathrm{E}-09$ \\
\hline CR 52 & $1.75 \mathrm{E}-01$ & $1.75 \mathrm{E}-01$ & $1.75 \mathrm{E}-01$ & $1.75 \mathrm{E}-01$ & $1.75 \mathrm{E}-01$ & $1.75 \mathrm{E}-01$ & $1.75 \mathrm{E}-01$ & $1.75 \mathrm{E}-01$ & $1.75 \mathrm{E}-01$ & $1.75 \mathrm{E}-01$ \\
\hline CR 53 & $2.06 \mathrm{E}-02$ & $2.06 \mathrm{E}-02$ & $2.06 \mathrm{E}-02$ & $2.06 \mathrm{E}-02$ & $2.06 \mathrm{E}-02$ & $2.06 \mathrm{E}-02$ & $2.06 \mathrm{E}-02$ & $2.06 \mathrm{E}-02$ & $2.06 \mathrm{E}-02$ & $2.06 \mathrm{E}-02$ \\
\hline CR 54 & $5.49 \mathrm{E}-03$ & $5.49 \mathrm{E}-03$ & $5.49 \mathrm{E}-03$ & $5.49 \mathrm{E}-03$ & $5.49 \mathrm{E}-03$ & $5.49 \mathrm{E}-03$ & $5.49 \mathrm{E}-03$ & $5.49 \mathrm{E}-03$ & $5.49 \mathrm{E}-03$ & $5.49 \mathrm{E}-03$ \\
\hline CR 55 & 3.07E-11 & $8.77 E-14$ & $0.00 \mathrm{E}+00$ & $0.00 \mathrm{E}+00$ & $0.00 \mathrm{E}+00$ & $0.00 \mathrm{E}+00$ & $0.00 \mathrm{E}+00$ & $0.00 \mathrm{E}+00$ & $0.00 \mathrm{E}+00$ & $0.00 \mathrm{E}+00$ \\
\hline MN 54 & $9.95 \mathrm{E}-07$ & $9.95 \mathrm{E}-07$ & $9.93 \mathrm{E}-07$ & $9.80 \mathrm{E}-07$ & $9.31 \mathrm{E}-07$ & $8.71 \mathrm{E}-07$ & $8.15 \mathrm{E}-07$ & $7.63 \mathrm{E}-07$ & $6.39 \mathrm{E}-07$ & $4.48 \mathrm{E}-07$ \\
\hline MN 55 & $3.45 \mathrm{E}-03$ & $3.45 \mathrm{E}-03$ & $3.45 \mathrm{E}-03$ & $3.45 \mathrm{E}-03$ & $3.45 \mathrm{E}-03$ & $3.45 \mathrm{E}-03$ & $3.45 \mathrm{E}-03$ & $3.45 \mathrm{E}-03$ & $3.45 \mathrm{E}-03$ & $3.45 \mathrm{E}-03$ \\
\hline MN 56 & $4.38 \mathrm{E}-08$ & $3.83 \mathrm{E}-08$ & $6.90 \mathrm{E}-11$ & $1.07 E-27$ & $0.00 \mathrm{E}+00$ & $0.00 \mathrm{E}+00$ & $0.00 \mathrm{E}+00$ & $0.00 \mathrm{E}+00$ & $0.00 \mathrm{E}+00$ & $0.00 \mathrm{E}+00$ \\
\hline MN 57 & $2.45 \mathrm{E}-14$ & $6.03 E-20$ & $0.00 \mathrm{E}+00$ & $0.00 \mathrm{E}+00$ & $0.00 \mathrm{E}+00$ & $0.00 \mathrm{E}+00$ & $0.00 \mathrm{E}+00$ & $0.00 \mathrm{E}+00$ & $0.00 \mathrm{E}+00$ & $0.00 \mathrm{E}+00$ \\
\hline FE 54 & $6.21 \mathrm{E}-03$ & $6.21 \mathrm{E}-03$ & $6.21 \mathrm{E}-03$ & $6.21 \mathrm{E}-03$ & $6.21 \mathrm{E}-03$ & $6.21 \mathrm{E}-03$ & $6.21 \mathrm{E}-03$ & $6.21 \mathrm{E}-03$ & $6.21 \mathrm{E}-03$ & $6.21 \mathrm{E}-03$ \\
\hline FE 55 & $1.69 \mathrm{E}-05$ & $1.69 \mathrm{E}-05$ & $1.68 \mathrm{E}-05$ & $1.68 \mathrm{E}-05$ & $1.65 \mathrm{E}-05$ & $1.61 \mathrm{E}-05$ & $1.58 \mathrm{E}-05$ & $1.54 \mathrm{E}-05$ & $1.46 \mathrm{E}-05$ & $1.30 \mathrm{E}-05$ \\
\hline FE 56 & $1.02 \mathrm{E}-01$ & $1.02 \mathrm{E}-01$ & $1.02 \mathrm{E}-01$ & $1.02 \mathrm{E}-01$ & $1.02 \mathrm{E}-01$ & $1.02 \mathrm{E}-01$ & $1.02 \mathrm{E}-01$ & $1.02 \mathrm{E}-01$ & $1.02 \mathrm{E}-01$ & $1.02 \mathrm{E}-01$ \\
\hline FE 57 & $2.71 \mathrm{E}-03$ & $2.71 \mathrm{E}-03$ & $2.71 \mathrm{E}-03$ & $2.71 \mathrm{E}-03$ & $2.71 \mathrm{E}-03$ & $2.71 \mathrm{E}-03$ & $2.71 \mathrm{E}-03$ & $2.71 \mathrm{E}-03$ & $2.71 \mathrm{E}-03$ & $2.71 \mathrm{E}-03$ \\
\hline FE 58 & $3.65 \mathrm{E}-04$ & $3.65 \mathrm{E}-04$ & $3.65 \mathrm{E}-04$ & $3.66 \mathrm{E}-04$ & $3.69 \mathrm{E}-04$ & $3.72 \mathrm{E}-04$ & $3.74 \mathrm{E}-04$ & $3.75 \mathrm{E}-04$ & $3.78 \mathrm{E}-04$ & $3.79 \mathrm{E}-04$ \\
\hline FE 59 & $1.30 \mathrm{E}-07$ & $1.30 \mathrm{E}-07$ & $1.28 \mathrm{E}-07$ & 1.17E-07 & $8.22 \mathrm{E}-08$ & $5.18 \mathrm{E}-08$ & $3.26 \mathrm{E}-08$ & $2.05 \mathrm{E}-08$ & 5.99E-09 & $5.09 \mathrm{E}-10$ \\
\hline $\mathrm{CO} 58$ & $1.44 \mathrm{E}-05$ & $1.44 \mathrm{E}-05$ & $1.42 \mathrm{E}-05$ & $1.34 \mathrm{E}-05$ & 1.07E-05 & $7.99 \mathrm{E}-06$ & $5.95 \mathrm{E}-06$ & 4.44E-06 & $2.03 \mathrm{E}-06$ & $4.23 \mathrm{E}-07$ \\
\hline $\mathrm{CO} 59$ & $9.56 \mathrm{E}-03$ & $9.56 \mathrm{E}-03$ & $9.56 \mathrm{E}-03$ & $9.56 \mathrm{E}-03$ & $9.56 \mathrm{E}-03$ & $9.56 \mathrm{E}-03$ & $9.56 \mathrm{E}-03$ & $9.56 \mathrm{E}-03$ & $9.56 \mathrm{E}-03$ & $9.56 \mathrm{E}-03$ \\
\hline
\end{tabular}


TEM-10200-1

$12 / 19 / 17$

Rev.08

Title: AS-RUN NEUTRONICS EVALUATION FOR THE GE HITACHI EXPERIMENT IN THE ATR ECAR NO.: $4740 \quad$ REV. NO.: 0

\begin{tabular}{|c|c|c|c|c|c|c|c|c|c|c|}
\hline \multicolumn{11}{|c|}{$1 \mathrm{~g}$ of Inconel } \\
\hline Isotone & EOI & $30.0 \mathrm{MI}$ & $1.0 \mathrm{D}$ & $7.0 \mathrm{D}$ & $30.0 \mathrm{D}$ & $60.0 \mathrm{D}$ & $90.0 \mathrm{D}$ & $120.0 \mathrm{D}$ & $200.0 \mathrm{D}$ & $360.0 \mathrm{D}$ \\
\hline tsolote & $\mathrm{g} / 1 \mathrm{~g}$ of incon & $\mathrm{g} / 1 \mathrm{~g}$ of incon & $\mathrm{g} / 1 \mathrm{~g}$ of incon & $\mathrm{g} / 1 \mathrm{~g}$ of incon & $\mathrm{g} / \mathrm{g}$ of incon & $\mathrm{g} / 1 \mathrm{~g}$ of incon & $\mathrm{g} / 1 \mathrm{~g}$ of incon & $\mathrm{g} / 1 \mathrm{~g}$ of incon & $\mathrm{g} / 1 \mathrm{~g}$ of incon & $\mathrm{g} / 1 \mathrm{~g}$ of incon \\
\hline $\mathrm{CO} 60$ & $4.64 \mathrm{E}-04$ & $4.64 \mathrm{E}-04$ & $4.64 \mathrm{E}-04$ & $4.63 \mathrm{E}-04$ & 4.59E-04 & $4.54 \mathrm{E}-04$ & $4.49 \mathrm{E}-04$ & 4.44E-04 & $4.32 \mathrm{E}-04$ & $4.08 \mathrm{E}-04$ \\
\hline $\mathrm{CO} 60 \mathrm{M}$ & $1.22 \mathrm{E}-08$ & $1.68 \mathrm{E}-09$ & $0.00 \mathrm{E}+00$ & $0.00 \mathrm{E}+00$ & $0.00 \mathrm{E}+00$ & $0.00 \mathrm{E}+00$ & $0.00 \mathrm{E}+00$ & $0.00 \mathrm{E}+00$ & $0.00 \mathrm{E}+00$ & $0.00 \mathrm{E}+00$ \\
\hline $\mathrm{CO} 61$ & $5.88 \mathrm{E}-10$ & $4.77 \mathrm{E}-10$ & $2.46 \mathrm{E}-14$ & $0.00 \mathrm{E}+00$ & $0.00 \mathrm{E}+00$ & $0.00 \mathrm{E}+00$ & $0.00 \mathrm{E}+00$ & $0.00 \mathrm{E}+00$ & $0.00 \mathrm{E}+00$ & $0.00 \mathrm{E}+00$ \\
\hline $\mathrm{CO} 62$ & $4.61 \mathrm{E}-14$ & 4.40E-20 & $0.00 \mathrm{E}+00$ & $0.00 \mathrm{E}+00$ & $0.00 \mathrm{E}+00$ & $0.00 \mathrm{E}+00$ & $0.00 \mathrm{E}+00$ & $0.00 \mathrm{E}+00$ & $0.00 \mathrm{E}+00$ & $0.00 \mathrm{E}+00$ \\
\hline NI 58 & 3.69E-01 & $3.69 \mathrm{E}-01$ & 3.69E-01 & $3.69 \mathrm{E}-01$ & $3.69 \mathrm{E}-01$ & 3.69E-01 & $3.69 \mathrm{E}-01$ & $3.69 \mathrm{E}-01$ & $3.69 \mathrm{E}-01$ & $3.69 \mathrm{E}-01$ \\
\hline NI 59 & $1.73 \mathrm{E}-03$ & $1.73 \mathrm{E}-03$ & $1.73 \mathrm{E}-03$ & $1.73 \mathrm{E}-03$ & $1.73 \mathrm{E}-03$ & $1.73 \mathrm{E}-03$ & $1.73 \mathrm{E}-03$ & $1.73 \mathrm{E}-03$ & $1.73 \mathrm{E}-03$ & $1.73 \mathrm{E}-03$ \\
\hline $\mathrm{NI} 60$ & $1.46 \mathrm{E}-01$ & $1.46 \mathrm{E}-01$ & $1.46 \mathrm{E}-01$ & $1.46 \mathrm{E}-01$ & $1.46 \mathrm{E}-01$ & $1.46 \mathrm{E}-01$ & $1.46 \mathrm{E}-01$ & $1.46 \mathrm{E}-01$ & $1.46 \mathrm{E}-01$ & $1.46 \mathrm{E}-01$ \\
\hline $\mathrm{NI} 61$ & $6.83 \mathrm{E}-03$ & $6.83 \mathrm{E}-03$ & $6.83 \mathrm{E}-03$ & $6.83 \mathrm{E}-03$ & $6.83 \mathrm{E}-03$ & $6.83 \mathrm{E}-03$ & $6.83 \mathrm{E}-03$ & $6.83 \mathrm{E}-03$ & $6.83 \mathrm{E}-03$ & $6.83 \mathrm{E}-03$ \\
\hline NI 62 & $2.06 \mathrm{E}-02$ & $2.06 \mathrm{E}-02$ & $2.06 \mathrm{E}-02$ & $2.06 \mathrm{E}-02$ & $2.06 \mathrm{E}-02$ & $2.06 \mathrm{E}-02$ & $2.06 \mathrm{E}-02$ & $2.06 \mathrm{E}-02$ & $2.06 \mathrm{E}-02$ & $2.06 \mathrm{E}-02$ \\
\hline NI 63 & $2.74 \mathrm{E}-04$ & $2.74 \mathrm{E}-04$ & $2.74 \mathrm{E}-04$ & $2.74 \mathrm{E}-04$ & $2.74 \mathrm{E}-04$ & $2.74 \mathrm{E}-04$ & $2.73 \mathrm{E}-04$ & $2.73 \mathrm{E}-04$ & $2.73 \mathrm{E}-04$ & $2.72 \mathrm{E}-04$ \\
\hline NI 64 & $5.45 \mathrm{E}-03$ & $5.45 \mathrm{E}-03$ & $5.45 \mathrm{E}-03$ & $5.45 \mathrm{E}-03$ & $5.45 \mathrm{E}-03$ & $5.45 \mathrm{E}-03$ & $5.45 \mathrm{E}-03$ & $5.45 \mathrm{E}-03$ & $5.45 \mathrm{E}-03$ & $5.45 \mathrm{E}-03$ \\
\hline NI 65 & $5.65 \mathrm{E}-09$ & 4.93E-09 & 7.69E-12 & 4.83E-29 & $0.00 \mathrm{E}+00$ & $0.00 \mathrm{E}+00$ & $0.00 \mathrm{E}+00$ & $0.00 \mathrm{E}+00$ & $0.00 \mathrm{E}+00$ & $0.00 \mathrm{E}+00$ \\
\hline NI 66 & $1.90 \mathrm{E}-12$ & 1.89E-12 & $1.40 \mathrm{E}-12$ & $2.25 \mathrm{E}-13$ & $2.04 \mathrm{E}-16$ & $2.19 \mathrm{E}-20$ & $2.35 \mathrm{E}-24$ & $2.53 \mathrm{E}-28$ & $0.00 \mathrm{E}+00$ & $0.00 \mathrm{E}+00$ \\
\hline CU 63 & $2.05 \mathrm{E}-03$ & $2.05 \mathrm{E}-03$ & $2.05 \mathrm{E}-03$ & $2.05 \mathrm{E}-03$ & $2.05 \mathrm{E}-03$ & $2.05 \mathrm{E}-03$ & $2.05 \mathrm{E}-03$ & $2.05 \mathrm{E}-03$ & $2.05 \mathrm{E}-03$ & $2.05 \mathrm{E}-03$ \\
\hline CU 64 & $3.51 \mathrm{E}-08$ & $3.42 \mathrm{E}-08$ & $9.48 \mathrm{E}-09$ & $3.66 \mathrm{E}-12$ & $3.02 \mathrm{E}-25$ & $0.00 \mathrm{E}+00$ & $0.00 \mathrm{E}+00$ & $0.00 \mathrm{E}+00$ & $0.00 \mathrm{E}+00$ & $0.00 \mathrm{E}+00$ \\
\hline CU 65 & $9.50 \mathrm{E}-04$ & $9.50 \mathrm{E}-04$ & $9.50 \mathrm{E}-04$ & $9.50 \mathrm{E}-04$ & 9.50E-04 & $9.50 \mathrm{E}-04$ & 9.50E-04 & 9.50E-04 & 9.50E-04 & 9.50E-04 \\
\hline CU 66 & $5.28 \mathrm{E}-11$ & $8.98 \mathrm{E}-13$ & $2.18 \mathrm{E}-15$ & $3.51 \mathrm{E}-16$ & $3.18 \mathrm{E}-19$ & $3.42 \mathrm{E}-23$ & 3.67E-27 & $3.94 \mathrm{E}-31$ & $0.00 \mathrm{E}+00$ & $0.00 \mathrm{E}+00$ \\
\hline CU 67 & $1.13 \mathrm{E}-13$ & $1.12 \mathrm{E}-13$ & $8.63 \mathrm{E}-14$ & $1.72 \mathrm{E}-14$ & $3.53 \mathrm{E}-17$ & $1.11 \mathrm{E}-20$ & $3.47 \mathrm{E}-24$ & 1.09E-27 & $0.00 \mathrm{E}+00$ & $0.00 \mathrm{E}+00$ \\
\hline ZN 64 & $3.75 \mathrm{E}-06$ & 3.75E-06 & $3.76 \mathrm{E}-06$ & 3.76E-06 & $3.76 \mathrm{E}-06$ & $3.76 \mathrm{E}-06$ & 3.76E-06 & $3.76 \mathrm{E}-06$ & $3.76 \mathrm{E}-06$ & $3.76 \mathrm{E}-06$ \\
\hline ZN 65 & $1.53 \mathrm{E}-09$ & 1.53E-09 & $1.52 \mathrm{E}-09$ & 1.50E-09 & $1.40 \mathrm{E}-09$ & $1.29 \mathrm{E}-09$ & $1.18 \mathrm{E}-09$ & $1.09 \mathrm{E}-09$ & $8.66 \mathrm{E}-10$ & $5.49 \mathrm{E}-10$ \\
\hline ZN 66 & $2.26 \mathrm{E}-06$ & $2.26 \mathrm{E}-06$ & $2.26 \mathrm{E}-06$ & $2.26 \mathrm{E}-06$ & $2.26 \mathrm{E}-06$ & $2.26 \mathrm{E}-06$ & $2.26 \mathrm{E}-06$ & $2.26 \mathrm{E}-06$ & $2.26 \mathrm{E}-06$ & $2.26 \mathrm{E}-06$ \\
\hline ZN 67 & $1.02 \mathrm{E}-09$ & $1.02 \mathrm{E}-09$ & $1.02 \mathrm{E}-09$ & 1.02E-09 & $1.02 \mathrm{E}-09$ & $1.02 \mathrm{E}-09$ & 1.02E-09 & $1.02 \mathrm{E}-09$ & 1.02E-09 & $1.02 \mathrm{E}-09$ \\
\hline ZN 68 & 3.63E-12 & 3.63E-12 & 3.63E-12 & 3.63E-12 & 3.63E-12 & 3.63E-12 & 3.63E-12 & 3.63E-12 & 3.63E-12 & $3.63 \mathrm{E}-12$ \\
\hline GA 69 & $1.55 \mathrm{E}-15$ & $1.55 \mathrm{E}-15$ & $1.55 \mathrm{E}-15$ & $1.56 \mathrm{E}-15$ & $1.56 \mathrm{E}-15$ & $1.56 \mathrm{E}-15$ & $1.56 \mathrm{E}-15$ & $1.56 \mathrm{E}-15$ & $1.56 \mathrm{E}-15$ & $1.56 \mathrm{E}-15$ \\
\hline SR 88 & $1.31 \mathrm{E}-14$ & $1.31 \mathrm{E}-14$ & $1.31 \mathrm{E}-14$ & $1.31 \mathrm{E}-14$ & $1.31 \mathrm{E}-14$ & $1.31 \mathrm{E}-14$ & $1.31 \mathrm{E}-14$ & $1.31 \mathrm{E}-14$ & $1.31 \mathrm{E}-14$ & $1.31 \mathrm{E}-14$ \\
\hline SR 89 & $6.84 \mathrm{E}-15$ & $6.83 \mathrm{E}-15$ & $6.74 \mathrm{E}-15$ & $6.21 \mathrm{E}-15$ & $4.53 \mathrm{E}-15$ & $3.00 \mathrm{E}-15$ & $1.99 \mathrm{E}-15$ & $1.32 \mathrm{E}-15$ & $4.39 \mathrm{E}-16$ & $4.88 \mathrm{E}-17$ \\
\hline SR 90 & 7.44E-15 & 7.44E-15 & 7.44E-15 & 7.44E-15 & 7.42E-15 & 7.41E-15 & $7.40 \mathrm{E}-15$ & $7.38 \mathrm{E}-15$ & 7.34E-15 & 7.27E-15 \\
\hline Y 89 & 4.23E-10 & 4.23E-10 & $4.25 \mathrm{E}-10$ & $4.31 \mathrm{E}-10$ & 4.33E-10 & 4.33E-10 & $4.33 \mathrm{E}-10$ & 4.33E-10 & 4.33E-10 & $4.33 \mathrm{E}-10$ \\
\hline Y 90 & $1.73 \mathrm{E}-10$ & $1.72 \mathrm{E}-10$ & $1.33 \mathrm{E}-10$ & 2.80E-11 & 7.08E-14 & 3.09E-17 & 1.87E-18 & 1.85E-18 & 1.84E-18 & 1.82E-18 \\
\hline Y 91 & $2.66 \mathrm{E}-13$ & $2.66 \mathrm{E}-13$ & $2.63 \mathrm{E}-13$ & $2.45 \mathrm{E}-13$ & $1.87 \mathrm{E}-13$ & $1.31 \mathrm{E}-13$ & $9.17 \mathrm{E}-14$ & $6.43 E-14$ & $2.49 \mathrm{E}-14$ & $3.74 \mathrm{E}-15$ \\
\hline ZR 89 & $9.30 \mathrm{E}-12$ & $9.26 \mathrm{E}-12$ & 7.52E-12 & $2.11 \mathrm{E}-12$ & $1.61 \mathrm{E}-14$ & 2.77E-17 & 4.77E-20 & $8.68 \mathrm{E}-23$ & $3.72 \mathrm{E}-30$ & $0.00 \mathrm{E}+00$ \\
\hline ZR 90 & $9.70 \mathrm{E}-09$ & 9.70E-09 & 9.74E-09 & 9.84E-09 & 9.87E-09 & 9.87E-09 & 9.87E-09 & 9.87E-09 & $9.87 \mathrm{E}-09$ & $9.87 \mathrm{E}-09$ \\
\hline ZR 91 & 5.89E-09 & 5.89E-09 & 5.89E-09 & 5.89E-09 & 5.89E-09 & 5.89E-09 & $5.89 \mathrm{E}-09$ & 5.89E-09 & 5.89E-09 & 5.89E-09 \\
\hline ZR 92 & $1.39 \mathrm{E}-07$ & $1.39 \mathrm{E}-07$ & $1.39 \mathrm{E}-07$ & $1.41 \mathrm{E}-07$ & 1.44E-07 & $1.45 \mathrm{E}-07$ & $1.45 \mathrm{E}-07$ & $1.45 \mathrm{E}-07$ & $1.45 \mathrm{E}-07$ & $1.45 \mathrm{E}-07$ \\
\hline ZR 93 & $1.01 \mathrm{E}-07$ & $1.01 \mathrm{E}-07$ & $1.01 \mathrm{E}-07$ & $1.01 \mathrm{E}-07$ & $1.01 \mathrm{E}-07$ & $1.01 \mathrm{E}-07$ & $1.01 \mathrm{E}-07$ & $1.01 \mathrm{E}-07$ & $1.01 \mathrm{E}-07$ & $1.01 \mathrm{E}-07$ \\
\hline ZR 94 & $1.29 \mathrm{E}-08$ & $1.29 \mathrm{E}-08$ & $1.29 \mathrm{E}-08$ & $1.29 \mathrm{E}-08$ & $1.29 \mathrm{E}-08$ & $1.29 \mathrm{E}-08$ & $1.29 \mathrm{E}-08$ & $1.29 \mathrm{E}-08$ & $1.29 \mathrm{E}-08$ & $1.29 \mathrm{E}-08$ \\
\hline ZR 95 & $6.09 \mathrm{E}-10$ & $6.09 \mathrm{E}-10$ & $6.02 \mathrm{E}-10$ & $5.64 \mathrm{E}-10$ & 4.40E-10 & $3.18 \mathrm{E}-10$ & $2.30 \mathrm{E}-10$ & $1.66 \mathrm{E}-10$ & $6.98 \mathrm{E}-11$ & $1.23 \mathrm{E}-11$ \\
\hline
\end{tabular}


TEM-10200-1

$12 / 19 / 17$

Rev.08

Title: AS-RUN NEUTRONICS EVALUATION FOR THE GE HITACHI EXPERIMENT IN THE ATR ECAR NO.: $4740 \quad$ REV. NO.: 0

\begin{tabular}{|c|c|c|c|c|c|c|c|c|c|c|}
\hline \multicolumn{11}{|c|}{$1 \mathrm{~g}$ of Inconel } \\
\hline & EOI & $30.0 \mathrm{MI}$ & $1.0 \mathrm{D}$ & $7.0 \mathrm{D}$ & $30.0 \mathrm{D}$ & $60.0 \mathrm{D}$ & $90.0 \mathrm{D}$ & $120.0 \mathrm{D}$ & $200.0 \mathrm{D}$ & $360.0 \mathrm{D}$ \\
\hline Isotope & $\mathrm{g} / 1 \mathrm{~g}$ of incon & $\mathrm{g} / 1 \mathrm{~g}$ of incon & $\mathrm{g} / 1 \mathrm{~g}$ of incon & $\mathrm{g} / 1 \mathrm{~g}$ of incon & $\mathrm{g} / 1 \mathrm{~g}$ of incon & $\mathrm{g} / 1 \mathrm{~g}$ of incon & $\mathrm{g} / 1 \mathrm{~g}$ of incon & $\mathrm{g} / 1 \mathrm{~g}$ of incon & $\mathrm{g} / 1 \mathrm{~g}$ of incon & $\mathrm{g} / 1 \mathrm{~g}$ of incon \\
\hline ZR 96 & $9.76 \mathrm{E}-13$ & $9.76 \mathrm{E}-13$ & $9.76 \mathrm{E}-13$ & $9.76 \mathrm{E}-13$ & $9.76 \mathrm{E}-13$ & $9.76 \mathrm{E}-13$ & $9.76 \mathrm{E}-13$ & $9.76 \mathrm{E}-13$ & $9.76 \mathrm{E}-13$ & $9.76 \mathrm{E}-13$ \\
\hline ZR 97 & $1.73 E-13$ & $1.69 \mathrm{E}-13$ & $6.45 \mathrm{E}-14$ & $1.76 \mathrm{E}-16$ & $2.59 \mathrm{E}-26$ & $0.00 \mathrm{E}+00$ & $0.00 E+00$ & $0.00 E+00$ & $0.00 \mathrm{E}+00$ & $0.00 \mathrm{E}+00$ \\
\hline NB 92 & $5.95 \mathrm{E}-09$ & $5.94 \mathrm{E}-09$ & $5.56 \mathrm{E}-09$ & $3.69 \mathrm{E}-09$ & $7.68 \mathrm{E}-10$ & $9.92 \mathrm{E}-11$ & $1.28 \mathrm{E}-11$ & $1.66 \mathrm{E}-12$ & $7.06 \mathrm{E}-15$ & $1.28 \mathrm{E}-19$ \\
\hline NB 93 & $5.47 \mathrm{E}-02$ & $5.47 \mathrm{E}-02$ & $5.47 \mathrm{E}-02$ & $5.47 \mathrm{E}-02$ & $5.47 \mathrm{E}-02$ & $5.47 \mathrm{E}-02$ & $5.47 \mathrm{E}-02$ & $5.47 \mathrm{E}-02$ & $5.47 \mathrm{E}-02$ & $5.47 \mathrm{E}-02$ \\
\hline NB 93M & $1.29 \mathrm{E}-14$ & $1.29 \mathrm{E}-14$ & $1.30 \mathrm{E}-14$ & $1.37 E-14$ & $1.64 \mathrm{E}-14$ & 1.99E-14 & $2.33 \mathrm{E}-14$ & $2.68 \mathrm{E}-14$ & $3.59 \mathrm{E}-14$ & $5.38 \mathrm{E}-14$ \\
\hline NB 94 & $2.23 \mathrm{E}-04$ & $2.23 \mathrm{E}-04$ & $2.23 \mathrm{E}-04$ & $2.23 \mathrm{E}-04$ & $2.23 \mathrm{E}-04$ & $2.23 \mathrm{E}-04$ & $2.23 \mathrm{E}-04$ & $2.23 \mathrm{E}-04$ & $2.23 \mathrm{E}-04$ & $2.23 \mathrm{E}-04$ \\
\hline NB 95 & $1.73 \mathrm{E}-06$ & $1.73 \mathrm{E}-06$ & $1.70 \mathrm{E}-06$ & $1.51 \mathrm{E}-06$ & $9.58 \mathrm{E}-07$ & $5.30 \mathrm{E}-07$ & $2.93 \mathrm{E}-07$ & $1.62 \mathrm{E}-07$ & $3.36 \mathrm{E}-08$ & $1.44 \mathrm{E}-09$ \\
\hline NB 95M & $2.39 \mathrm{E}-13$ & $2.39 \mathrm{E}-13$ & $2.39 \mathrm{E}-13$ & $2.32 \mathrm{E}-13$ & $1.84 \mathrm{E}-13$ & $1.33 \mathrm{E}-13$ & $9.61 \mathrm{E}-14$ & $6.94 \mathrm{E}-14$ & $2.92 \mathrm{E}-14$ & $5.16 \mathrm{E}-15$ \\
\hline NB 96 & $1.16 \mathrm{E}-10$ & $1.14 \mathrm{E}-10$ & $5.68 \mathrm{E}-11$ & $7.90 \mathrm{E}-13$ & $6.05 \mathrm{E}-20$ & $3.16 \mathrm{E}-29$ & $0.00 \mathrm{E}+00$ & $0.00 \mathrm{E}+00$ & $0.00 \mathrm{E}+00$ & $0.00 \mathrm{E}+00$ \\
\hline NB 97 & $4.35 \mathrm{E}-13$ & $3.29 \mathrm{E}-13$ & $4.61 \mathrm{E}-15$ & $1.26 \mathrm{E}-17$ & $1.98 \mathrm{E}-27$ & $0.00 \mathrm{E}+00$ & $0.00 \mathrm{E}+00$ & $0.00 \mathrm{E}+00$ & $0.00 \mathrm{E}+00$ & $0.00 \mathrm{E}+00$ \\
\hline MO 92 & $4.68 \mathrm{E}-03$ & $4.68 \mathrm{E}-03$ & $4.68 \mathrm{E}-03$ & $4.68 \mathrm{E}-03$ & $4.68 \mathrm{E}-03$ & $4.68 \mathrm{E}-03$ & $4.68 \mathrm{E}-03$ & $4.68 \mathrm{E}-03$ & $4.68 \mathrm{E}-03$ & $4.68 \mathrm{E}-03$ \\
\hline MO 93M & $4.91 \mathrm{E}-11$ & 4.66E-11 & $4.33 \mathrm{E}-12$ & $2.03 E-18$ & $0.00 \mathrm{E}+00$ & $0.00 \mathrm{E}+00$ & $0.00 \mathrm{E}+00$ & $0.00 \mathrm{E}+00$ & $0.00 \mathrm{E}+00$ & $0.00 E+00$ \\
\hline MO 93 & $8.04 \mathrm{E}-07$ & $8.04 \mathrm{E}-07$ & $8.05 E-07$ & $8.05 E-07$ & $8.04 \mathrm{E}-07$ & 8.04E-07 & 8.04E-07 & $8.04 \mathrm{E}-07$ & 8.04E-07 & $8.04 \mathrm{E}-07$ \\
\hline MO 94 & $3.00 \mathrm{E}-03$ & $3.00 \mathrm{E}-03$ & $3.00 \mathrm{E}-03$ & $3.00 \mathrm{E}-03$ & $3.00 \mathrm{E}-03$ & $3.00 \mathrm{E}-03$ & $3.00 \mathrm{E}-03$ & $3.00 \mathrm{E}-03$ & $3.00 \mathrm{E}-03$ & $3.00 \mathrm{E}-03$ \\
\hline MO 95 & $4.98 \mathrm{E}-03$ & $4.98 \mathrm{E}-03$ & $4.98 \mathrm{E}-03$ & $4.98 \mathrm{E}-03$ & $4.98 \mathrm{E}-03$ & $4.98 \mathrm{E}-03$ & $4.98 \mathrm{E}-03$ & $4.98 \mathrm{E}-03$ & $4.98 \mathrm{E}-03$ & $4.98 \mathrm{E}-03$ \\
\hline MO 96 & $5.69 \mathrm{E}-03$ & $5.69 \mathrm{E}-03$ & $5.69 \mathrm{E}-03$ & $5.69 \mathrm{E}-03$ & 5.69E-03 & $5.69 \mathrm{E}-03$ & $5.69 \mathrm{E}-03$ & 5.69E-03 & $5.69 \mathrm{E}-03$ & $5.69 \mathrm{E}-03$ \\
\hline MO 97 & $3.22 \mathrm{E}-03$ & $3.22 \mathrm{E}-03$ & $3.22 \mathrm{E}-03$ & $3.22 \mathrm{E}-03$ & $3.22 \mathrm{E}-03$ & $3.22 \mathrm{E}-03$ & $3.22 \mathrm{E}-03$ & $3.22 \mathrm{E}-03$ & $3.22 \mathrm{E}-03$ & $3.22 \mathrm{E}-03$ \\
\hline MO 98 & $8.12 \mathrm{E}-03$ & $8.12 \mathrm{E}-03$ & $8.12 \mathrm{E}-03$ & $8.12 \mathrm{E}-03$ & $8.12 \mathrm{E}-03$ & $8.12 \mathrm{E}-03$ & $8.12 \mathrm{E}-03$ & $8.12 \mathrm{E}-03$ & $8.12 \mathrm{E}-03$ & $8.12 \mathrm{E}-03$ \\
\hline MO 99 & $3.52 \mathrm{E}-07$ & $3.50 \mathrm{E}-07$ & $2.74 \mathrm{E}-07$ & $6.03 \mathrm{E}-08$ & $1.83 \mathrm{E}-10$ & $9.53 \mathrm{E}-14$ & $4.96 \mathrm{E}-17$ & $2.58 \mathrm{E}-20$ & $4.51 \mathrm{E}-29$ & $0.00 \mathrm{E}+00$ \\
\hline MO100 & $3.29 E-03$ & $3.29 \mathrm{E}-03$ & $3.29 E-03$ & $3.29 \mathrm{E}-03$ & $3.29 \mathrm{E}-03$ & $3.29 \mathrm{E}-03$ & $3.29 \mathrm{E}-03$ & $3.29 \mathrm{E}-03$ & $3.29 \mathrm{E}-03$ & $3.29 \mathrm{E}-03$ \\
\hline MO101 & $3.30 \mathrm{E}-10$ & 7.96E-11 & $0.00 \mathrm{E}+00$ & $0.00 \mathrm{E}+00$ & $0.00 \mathrm{E}+00$ & $0.00 \mathrm{E}+00$ & $0.00 \mathrm{E}+00$ & $0.00 E+00$ & $0.00 \mathrm{E}+00$ & $0.00 \mathrm{E}+00$ \\
\hline TC 99 & $2.28 \mathrm{E}-06$ & $2.28 \mathrm{E}-06$ & $2.29 \mathrm{E}-06$ & $2.31 \mathrm{E}-06$ & $2.32 \mathrm{E}-06$ & 2.32E-06 & $2.32 \mathrm{E}-06$ & $2.32 \mathrm{E}-06$ & $2.32 \mathrm{E}-06$ & $2.32 \mathrm{E}-06$ \\
\hline TC100 & $2.53 \mathrm{E}-13$ & $0.00 \mathrm{E}+00$ & $0.00 \mathrm{E}+00$ & $0.00 \mathrm{E}+00$ & $0.00 \mathrm{E}+00$ & $0.00 \mathrm{E}+00$ & $0.00 \mathrm{E}+00$ & $0.00 \mathrm{E}+00$ & $0.00 \mathrm{E}+00$ & $0.00 \mathrm{E}+00$ \\
\hline TC101 & $3.21 \mathrm{E}-10$ & $1.85 \mathrm{E}-10$ & $0.00 \mathrm{E}+00$ & $0.00 \mathrm{E}+00$ & $0.00 \mathrm{E}+00$ & $0.00 \mathrm{E}+00$ & $0.00 \mathrm{E}+00$ & $0.00 \mathrm{E}+00$ & $0.00 \mathrm{E}+00$ & $0.00 \mathrm{E}+00$ \\
\hline RU 99 & $2.19 \mathrm{E}-12$ & $2.19 \mathrm{E}-12$ & $2.21 \mathrm{E}-12$ & $2.33 \mathrm{E}-12$ & $2.81 \mathrm{E}-12$ & 3.43E-12 & $4.05 \mathrm{E}-12$ & 4.67E-12 & $6.32 \mathrm{E}-12$ & $9.63 \mathrm{E}-12$ \\
\hline RU100 & $1.05 \mathrm{E}-07$ & $1.05 \mathrm{E}-07$ & $1.05 \mathrm{E}-07$ & $1.05 \mathrm{E}-07$ & $1.05 \mathrm{E}-07$ & $1.05 E-07$ & $1.05 \mathrm{E}-07$ & $1.05 \mathrm{E}-07$ & $1.05 \mathrm{E}-07$ & $1.05 \mathrm{E}-07$ \\
\hline RU101 & $4.87 E-06$ & 4.87E-06 & 4.87E-06 & $4.87 \mathrm{E}-06$ & 4.87E-06 & 4.87E-06 & 4.87E-06 & 4.87E-06 & 4.87E-06 & 4.87E-06 \\
\hline RU102 & $7.30 \mathrm{E}-08$ & $7.30 \mathrm{E}-08$ & $7.30 \mathrm{E}-08$ & $7.30 \mathrm{E}-08$ & 7.30E-08 & $7.30 \mathrm{E}-08$ & $7.30 \mathrm{E}-08$ & 7.30E-08 & $7.30 \mathrm{E}-08$ & $7.30 \mathrm{E}-08$ \\
\hline RU103 & $3.06 \mathrm{E}-11$ & $3.06 \mathrm{E}-11$ & $3.01 \mathrm{E}-11$ & $2.71 \mathrm{E}-11$ & $1.80 \mathrm{E}-11$ & $1.06 \mathrm{E}-11$ & $6.26 \mathrm{E}-12$ & 3.69E-12 & $8.99 \mathrm{E}-13$ & $5.34 \mathrm{E}-14$ \\
\hline RU104 & $2.37 \mathrm{E}-13$ & 2.37E-13 & $2.37 \mathrm{E}-13$ & $2.37 \mathrm{E}-13$ & 2.37E-13 & $2.37 \mathrm{E}-13$ & $2.37 \mathrm{E}-13$ & 2.37E-13 & $2.37 \mathrm{E}-13$ & $2.37 \mathrm{E}-13$ \\
\hline RH103 & $3.09 E-12$ & 3.09E-12 & $3.14 \mathrm{E}-12$ & 3.44E-12 & 4.34E-12 & $5.08 \mathrm{E}-12$ & $5.51 \mathrm{E}-12$ & 5.77E-12 & $6.05 E-12$ & $6.13 \mathrm{E}-12$ \\
\hline PD104 & $2.74 \mathrm{E}-13$ & $2.74 \mathrm{E}-13$ & $2.74 \mathrm{E}-13$ & $2.74 \mathrm{E}-13$ & $2.74 \mathrm{E}-13$ & $2.74 \mathrm{E}-13$ & $2.74 \mathrm{E}-13$ & $2.74 \mathrm{E}-13$ & $2.74 \mathrm{E}-13$ & $2.74 \mathrm{E}-13$ \\
\hline TA180 & 2.92E-08 & 2.92E-08 & $2.92 \mathrm{E}-08$ & $2.92 \mathrm{E}-08$ & $2.92 \mathrm{E}-08$ & 2.92E-08 & $2.92 \mathrm{E}-08$ & $2.92 \mathrm{E}-08$ & $2.92 \mathrm{E}-08$ & $2.92 \mathrm{E}-08$ \\
\hline TA181 & 4.13E-04 & 4.13E-04 & 4.13E-04 & 4.13E-04 & 4.13E-04 & 4.13E-04 & 4.13E-04 & 4.13E-04 & 4.13E-04 & 4.13E-04 \\
\hline TA182 & 9.93E-06 & 9.93E-06 & 9.87E-06 & $9.52 \mathrm{E}-06$ & $8.29 \mathrm{E}-06$ & $6.92 \mathrm{E}-06$ & $5.77 \mathrm{E}-06$ & $4.82 \mathrm{E}-06$ & 2.97E-06 & $1.13 \mathrm{E}-06$ \\
\hline TA182M & $2.94 \mathrm{E}-12$ & $8.34 \mathrm{E}-13$ & $0.00 \mathrm{E}+00$ & $0.00 \mathrm{E}+00$ & $0.00 \mathrm{E}+00$ & $0.00 E+00$ & $0.00 \mathrm{E}+00$ & $0.00 \mathrm{E}+00$ & $0.00 E+00$ & $0.00 E+00$ \\
\hline TA183 & $2.32 \mathrm{E}-06$ & $2.32 \mathrm{E}-06$ & 2.03E-06 & $8.97 \mathrm{E}-07$ & $3.94 \mathrm{E}-08$ & $6.67 \mathrm{E}-10$ & 1.13E-11 & $1.92 \mathrm{E}-13$ & 3.64E-18 & $1.31 \mathrm{E}-27$ \\
\hline
\end{tabular}


TEM-10200-1

$12 / 19 / 17$

Rev.08

Title: AS-RUN NEUTRONICS EVALUATION FOR THE GE HITACHI EXPERIMENT IN THE ATR

ECAR NO.:

\begin{tabular}{|c|c|c|c|c|c|c|c|c|c|c|}
\hline \multicolumn{11}{|c|}{$1 \mathrm{~g}$ of Inconel } \\
\hline \multirow{2}{*}{ Isotope } & EOI & $30.0 \mathrm{MI}$ & $1.0 \mathrm{D}$ & $7.0 \mathrm{D}$ & $30.0 \mathrm{D}$ & $60.0 \mathrm{D}$ & $90.0 \mathrm{D}$ & $120.0 \mathrm{D}$ & $200.0 \mathrm{D}$ & $360.0 \mathrm{D}$ \\
\hline & $\mathrm{g} / 1 \mathrm{~g}$ of incon & $\mathrm{g} / 1 \mathrm{~g}$ of incon & $\mathrm{g} / 1 \mathrm{~g}$ of incon & $\mathrm{g} / 1 \mathrm{~g}$ of incon & $\mathrm{g} / 1 \mathrm{~g}$ of incon & $\mathrm{g} / 1 \mathrm{~g}$ of incon & $\mathrm{g} / 1 \mathrm{~g}$ of incon & $\mathrm{g} / 1 \mathrm{~g}$ of incon & $\mathrm{g} / 1 \mathrm{~g}$ of incon & $\mathrm{g} / 1 \mathrm{~g}$ of incon \\
\hline W182 & 1.17E-05 & 1.17E-05 & 1.17E-05 & $1.21 \mathrm{E}-05$ & $1.33 \mathrm{E}-05$ & $1.47 \mathrm{E}-05$ & $1.58 \mathrm{E}-05$ & $1.68 \mathrm{E}-05$ & $1.86 \mathrm{E}-05$ & $2.04 \mathrm{E}-05$ \\
\hline W183M & $2.12 \mathrm{E}-15$ & $0.00 \mathrm{E}+00$ & $0.00 \mathrm{E}+00$ & $0.00 \mathrm{E}+00$ & $0.00 \mathrm{E}+00$ & $0.00 \mathrm{E}+00$ & $0.00 \mathrm{E}+00$ & $0.00 \mathrm{E}+00$ & $0.00 \mathrm{E}+00$ & $0.00 \mathrm{E}+00$ \\
\hline W183 & $6.17 \mathrm{E}-05$ & $6.17 \mathrm{E}-05$ & $6.20 \mathrm{E}-05$ & $6.31 \mathrm{E}-05$ & $6.40 \mathrm{E}-05$ & $6.40 \mathrm{E}-05$ & $6.40 \mathrm{E}-05$ & $6.40 \mathrm{E}-05$ & $6.40 \mathrm{E}-05$ & $6.40 \mathrm{E}-05$ \\
\hline W184 & $2.59 \mathrm{E}-06$ & $2.59 \mathrm{E}-06$ & $2.59 \mathrm{E}-06$ & $2.59 \mathrm{E}-06$ & $2.59 \mathrm{E}-06$ & $2.59 \mathrm{E}-06$ & $2.59 \mathrm{E}-06$ & $2.59 \mathrm{E}-06$ & $2.59 \mathrm{E}-06$ & $2.59 \mathrm{E}-06$ \\
\hline W185 & $2.55 \mathrm{E}-09$ & $2.55 \mathrm{E}-09$ & $2.53 \mathrm{E}-09$ & $2.39 \mathrm{E}-09$ & $1.94 \mathrm{E}-09$ & 1.47E-09 & $1.11 \mathrm{E}-09$ & $8.43 \mathrm{E}-10$ & $4.03 \mathrm{E}-10$ & $9.20 \mathrm{E}-11$ \\
\hline W186 & $6.36 \mathrm{E}-12$ & $6.36 \mathrm{E}-12$ & $6.50 \mathrm{E}-12$ & $6.98 \mathrm{E}-12$ & $7.22 \mathrm{E}-12$ & $7.22 \mathrm{E}-12$ & $7.22 \mathrm{E}-12$ & $7.22 \mathrm{E}-12$ & $7.22 \mathrm{E}-12$ & $7.22 \mathrm{E}-12$ \\
\hline W187 & $6.20 \mathrm{E}-15$ & $6.11 \mathrm{E}-15$ & $3.09 \mathrm{E}-15$ & $4.74 \mathrm{E}-17$ & $5.29 \mathrm{E}-24$ & $4.52 E-33$ & $0.00 \mathrm{E}+00$ & $0.00 \mathrm{E}+00$ & $0.00 \mathrm{E}+00$ & $0.00 \mathrm{E}+00$ \\
\hline RE185 & $1.13 \mathrm{E}-09$ & $1.13 \mathrm{E}-09$ & $1.16 \mathrm{E}-09$ & $1.29 \mathrm{E}-09$ & $1.75 \mathrm{E}-09$ & $2.22 \mathrm{E}-09$ & $2.57 \mathrm{E}-09$ & $2.84 \mathrm{E}-09$ & $3.28 \mathrm{E}-09$ & $3.59 \mathrm{E}-09$ \\
\hline RE186 & $1.33 \mathrm{E}-11$ & $1.32 \mathrm{E}-11$ & $1.10 \mathrm{E}-11$ & $3.67 \mathrm{E}-12$ & $5.39 \mathrm{E}-14$ & $2.19 \mathrm{E}-16$ & $8.89 \mathrm{E}-19$ & $3.60 \mathrm{E}-21$ & $1.51 \mathrm{E}-27$ & $0.00 \mathrm{E}+00$ \\
\hline RE187 & $1.46 \mathrm{E}-13$ & $1.46 \mathrm{E}-13$ & $1.49 \mathrm{E}-13$ & $1.52 \mathrm{E}-13$ & $1.52 \mathrm{E}-13$ & $1.52 \mathrm{E}-13$ & $1.52 \mathrm{E}-13$ & $1.52 \mathrm{E}-13$ & $1.52 \mathrm{E}-13$ & $1.52 \mathrm{E}-13$ \\
\hline OS186 & $9.36 \mathrm{E}-11$ & $9.37 \mathrm{E}-11$ & $9.57 \mathrm{E}-11$ & $1.03 \mathrm{E}-10$ & 1.06E-10 & $1.06 \mathrm{E}-10$ & $1.06 \mathrm{E}-10$ & $1.06 \mathrm{E}-10$ & $1.06 \mathrm{E}-10$ & $1.06 \mathrm{E}-10$ \\
\hline OS188 & $2.75 \mathrm{E}-15$ & $2.75 \mathrm{E}-15$ & $2.81 \mathrm{E}-15$ & $2.87 \mathrm{E}-15$ & $2.98 \mathrm{E}-15$ & $3.09 \mathrm{E}-15$ & $3.17 \mathrm{E}-15$ & $3.22 \mathrm{E}-15$ & $3.32 \mathrm{E}-15$ & $3.38 \mathrm{E}-15$ \\
\hline SUMTOT & $1.00 \mathrm{E}+00$ & $1.00 \mathrm{E}+00$ & $1.00 \mathrm{E}+00$ & $1.00 \mathrm{E}+00$ & $1.00 \mathrm{E}+00$ & $1.00 \mathrm{E}+00$ & $1.00 \mathrm{E}+00$ & $1.00 \mathrm{E}+00$ & $1.00 \mathrm{E}+00$ & $1.00 \mathrm{E}+00$ \\
\hline OTOTAL & $1.00 \mathrm{E}+00$ & $1.00 \mathrm{E}+00$ & $1.00 \mathrm{E}+00$ & $1.00 \mathrm{E}+00$ & $1.00 \mathrm{E}+00$ & $1.00 \mathrm{E}+00$ & $1.00 \mathrm{E}+00$ & $1.00 \mathrm{E}+00$ & $1.00 \mathrm{E}+00$ & $1.00 \mathrm{E}+00$ \\
\hline
\end{tabular}

Table 19. $\mathrm{Ci} / 1 \mathrm{~g}$ of $316 \mathrm{SS}$

\begin{tabular}{|c|c|c|c|c|c|c|c|c|c|c|}
\hline \multicolumn{11}{|c|}{$1 \mathrm{~g}$ of $316 \mathrm{SS}$} \\
\hline \multirow{2}{*}{ Isotope } & EOI & $30.0 \mathrm{MI}$ & $1.0 \mathrm{D}$ & $7.0 \mathrm{D}$ & $30.0 \mathrm{D}$ & $60.0 \mathrm{D}$ & $90.0 \mathrm{D}$ & $120.0 \mathrm{D}$ & $200.0 \mathrm{D}$ & $360.0 \mathrm{D}$ \\
\hline & $\mathrm{Ci} / 1 \mathrm{~g}$ of $316 \mathrm{SS}$ & $\mathrm{Ci} / 1 \mathrm{~g}$ of $316 \mathrm{SS}$ & $\mathrm{Ci} / 1 \mathrm{~g}$ of $316 \mathrm{SS}$ & $\mathrm{Ci} / 1 \mathrm{~g}$ of $316 \mathrm{SS}$ & $\mathrm{Ci} / 1 \mathrm{~g}$ of $316 \mathrm{SS}$ & $\mathrm{Ci} / 1 \mathrm{~g}$ of $316 \mathrm{SS}$ & $\mathrm{Ci} / 1 \mathrm{~g}$ of $316 \mathrm{SS}$ & $\mathrm{Ci} / 1 \mathrm{~g}$ of $316 \mathrm{SS}$ & $\mathrm{Ci} / 1 \mathrm{~g}$ of $316 \mathrm{SS}$ & $\mathrm{Ci} / 1 \mathrm{~g}$ of $316 \mathrm{SS}$ \\
\hline $\mathrm{H} 3$ & $1.22 \mathrm{E}-10$ & $1.22 \mathrm{E}-10$ & $1.22 \mathrm{E}-10$ & $1.22 \mathrm{E}-10$ & $1.21 \mathrm{E}-10$ & $1.21 \mathrm{E}-10$ & 1.20E-10 & 1.20E-10 & $1.18 \mathrm{E}-10$ & 1.15E-10 \\
\hline HE 6 & $3.17 E-08$ & $0.00 \mathrm{E}+00$ & $0.00 \mathrm{E}+00$ & $0.00 \mathrm{E}+00$ & $0.00 \mathrm{E}+00$ & $0.00 E+00$ & $0.00 \mathrm{E}+00$ & $0.00 E+00$ & $0.00 \mathrm{E}+00$ & $0.00 \mathrm{E}+00$ \\
\hline LI 8 & 4.44E-10 & $0.00 \mathrm{E}+00$ & $0.00 \mathrm{E}+00$ & $0.00 \mathrm{E}+00$ & $0.00 \mathrm{E}+00$ & $0.00 \mathrm{E}+00$ & $0.00 \mathrm{E}+00$ & $0.00 \mathrm{E}+00$ & $0.00 \mathrm{E}+00$ & $0.00 \mathrm{E}+00$ \\
\hline BE 8 & $5.50 \mathrm{E}-08$ & $0.00 \mathrm{E}+00$ & $0.00 \mathrm{E}+00$ & $0.00 \mathrm{E}+00$ & $0.00 \mathrm{E}+00$ & $0.00 \mathrm{E}+00$ & $0.00 \mathrm{E}+00$ & $0.00 \mathrm{E}+00$ & $0.00 \mathrm{E}+00$ & $0.00 \mathrm{E}+00$ \\
\hline $\mathrm{BE} 10$ & $4.95 \mathrm{E}-13$ & $4.95 \mathrm{E}-13$ & $4.95 \mathrm{E}-13$ & $4.95 \mathrm{E}-13$ & $4.95 \mathrm{E}-13$ & $4.95 \mathrm{E}-13$ & $4.95 \mathrm{E}-13$ & $4.95 \mathrm{E}-13$ & $4.95 \mathrm{E}-13$ & $4.95 \mathrm{E}-13$ \\
\hline BE 11 & $1.75 \mathrm{E}-12$ & $0.00 \mathrm{E}+00$ & $0.00 \mathrm{E}+00$ & $0.00 \mathrm{E}+00$ & $0.00 \mathrm{E}+00$ & $0.00 E+00$ & $0.00 \mathrm{E}+00$ & $0.00 E+00$ & $0.00 \mathrm{E}+00$ & $0.00 \mathrm{E}+00$ \\
\hline B 12 & $9.53 \mathrm{E}-08$ & $0.00 \mathrm{E}+00$ & $0.00 \mathrm{E}+00$ & $0.00 \mathrm{E}+00$ & $0.00 \mathrm{E}+00$ & $0.00 \mathrm{E}+00$ & $0.00 \mathrm{E}+00$ & $0.00 \mathrm{E}+00$ & $0.00 \mathrm{E}+00$ & $0.00 \mathrm{E}+00$ \\
\hline C 14 & $8.05 \mathrm{E}-06$ & $8.05 \mathrm{E}-06$ & $8.05 \mathrm{E}-06$ & $8.05 \mathrm{E}-06$ & $8.05 \mathrm{E}-06$ & $8.05 \mathrm{E}-06$ & $8.05 \mathrm{E}-06$ & $8.05 \mathrm{E}-06$ & $8.05 \mathrm{E}-06$ & $8.05 \mathrm{E}-06$ \\
\hline C 15 & 1.47E-09 & $0.00 \mathrm{E}+00$ & $0.00 \mathrm{E}+00$ & $0.00 \mathrm{E}+00$ & $0.00 \mathrm{E}+00$ & $0.00 \mathrm{E}+00$ & $0.00 \mathrm{E}+00$ & $0.00 \mathrm{E}+00$ & $0.00 \mathrm{E}+00$ & $0.00 \mathrm{E}+00$ \\
\hline N 16 & $5.06 \mathrm{E}-09$ & $0.00 \mathrm{E}+00$ & $0.00 \mathrm{E}+00$ & $0.00 \mathrm{E}+00$ & $0.00 \mathrm{E}+00$ & $0.00 E+00$ & $0.00 E+00$ & $0.00 \mathrm{E}+00$ & $0.00 \mathrm{E}+00$ & $0.00 \mathrm{E}+00$ \\
\hline NE 23 & $1.94 \mathrm{E}-09$ & $5.46 \mathrm{E}-24$ & $0.00 \mathrm{E}+00$ & $0.00 \mathrm{E}+00$ & $0.00 \mathrm{E}+00$ & $0.00 \mathrm{E}+00$ & $0.00 \mathrm{E}+00$ & $0.00 \mathrm{E}+00$ & $0.00 \mathrm{E}+00$ & $0.00 \mathrm{E}+00$ \\
\hline NA 24 & $5.21 \mathrm{E}-12$ & $5.09 \mathrm{E}-12$ & $1.72 \mathrm{E}-12$ & $2.21 \mathrm{E}-15$ & $1.85 \mathrm{E}-26$ & $0.00 \mathrm{E}+00$ & $0.00 \mathrm{E}+00$ & $0.00 \mathrm{E}+00$ & $0.00 \mathrm{E}+00$ & $0.00 \mathrm{E}+00$ \\
\hline NA 24M & $3.70 \mathrm{E}-13$ & $0.00 \mathrm{E}+00$ & $0.00 \mathrm{E}+00$ & $0.00 \mathrm{E}+00$ & $0.00 \mathrm{E}+00$ & $0.00 \mathrm{E}+00$ & $0.00 \mathrm{E}+00$ & $0.00 \mathrm{E}+00$ & $0.00 \mathrm{E}+00$ & $0.00 \mathrm{E}+00$ \\
\hline NA 25 & $2.94 \mathrm{E}-09$ & $2.38 \mathrm{E}-18$ & $0.00 \mathrm{E}+00$ & $0.00 \mathrm{E}+00$ & $0.00 \mathrm{E}+00$ & $0.00 \mathrm{E}+00$ & $0.00 \mathrm{E}+00$ & $0.00 \mathrm{E}+00$ & $0.00 \mathrm{E}+00$ & $0.00 \mathrm{E}+00$ \\
\hline MG 27 & $2.19 \mathrm{E}-06$ & $2.43 \mathrm{E}-07$ & $0.00 \mathrm{E}+00$ & $0.00 \mathrm{E}+00$ & $0.00 \mathrm{E}+00$ & $0.00 \mathrm{E}+00$ & $0.00 \mathrm{E}+00$ & $0.00 \mathrm{E}+00$ & $0.00 \mathrm{E}+00$ & $0.00 \mathrm{E}+00$ \\
\hline
\end{tabular}


TEM-10200-1

$12 / 19 / 17$

Rev.08

Title:

AS-RUN NEUTRONICS EVALUATION FOR THE GE HITACHI EXPERIMENT IN THE ATR

\begin{tabular}{llllllll} 
ECAR NO.: & 4740 & REV. NO.: & 0 & PROJECT NO.: & 32535 & Date: & $12 / 02 / 2019$ \\
\hline
\end{tabular}

\begin{tabular}{|c|c|c|c|c|c|c|c|c|c|c|}
\hline \multicolumn{11}{|c|}{$1 \mathrm{~g}$ of $316 \mathrm{SS}$} \\
\hline Ilcoton & EOI & $30.0 \mathrm{MI}$ & $1.0 \mathrm{D}$ & $7.0 \mathrm{D}$ & $30.0 \mathrm{D}$ & $60.0 \mathrm{D}$ & $90.0 \mathrm{D}$ & $120.0 \mathrm{D}$ & $200.0 \mathrm{D}$ & $360.0 \mathrm{D}$ \\
\hline Isotope & $\mathrm{Ci} / 1 \mathrm{~g}$ of $316 \mathrm{SS}$ & $\mathrm{Ci} / 1 \mathrm{~g}$ of $316 \mathrm{SS}$ & $\mathrm{Ci} / 1 \mathrm{~g}$ of $316 \mathrm{SS}$ & $\mathrm{Ci} / 1 \mathrm{~g}$ of $316 \mathrm{SS}$ & $\mathrm{Ci} / 1 \mathrm{~g}$ of $316 \mathrm{SS}$ & $\mathrm{Ci} / 1 \mathrm{~g}$ of $316 \mathrm{SS}$ & $\mathrm{Ci} / 1 \mathrm{~g}$ of $316 \mathrm{SS}$ & $\mathrm{Ci} / 1 \mathrm{~g}$ of $316 \mathrm{SS}$ & $\mathrm{Ci} / 1 \mathrm{~g}$ of $316 \mathrm{SS}$ & $\mathrm{Ci} / 1 \mathrm{~g}$ of $316 \mathrm{SS}$ \\
\hline AL 28 & $2.65 \mathrm{E}-03$ & $2.46 \mathrm{E}-07$ & $1.58 \mathrm{E}-15$ & $1.33 \mathrm{E}-17$ & $0.00 \mathrm{E}+00$ & $0.00 \mathrm{E}+00$ & $0.00 \mathrm{E}+00$ & $0.00 \mathrm{E}+00$ & $0.00 \mathrm{E}+00$ & $0.00 \mathrm{E}+00$ \\
\hline AL 29 & $8.91 \mathrm{E}-05$ & $3.67 E-06$ & $0.00 \mathrm{E}+00$ & $0.00 \mathrm{E}+00$ & $0.00 \mathrm{E}+00$ & $0.00 \mathrm{E}+00$ & $0.00 \mathrm{E}+00$ & $0.00 \mathrm{E}+00$ & $0.00 \mathrm{E}+00$ & $0.00 \mathrm{E}+00$ \\
\hline AL 30 & $8.62 \mathrm{E}-08$ & $0.00 \mathrm{E}+00$ & $0.00 \mathrm{E}+00$ & $0.00 \mathrm{E}+00$ & $0.00 \mathrm{E}+00$ & $0.00 \mathrm{E}+00$ & $0.00 \mathrm{E}+00$ & $0.00 \mathrm{E}+00$ & $0.00 \mathrm{E}+00$ & $0.00 \mathrm{E}+00$ \\
\hline SI 31 & $1.99 \mathrm{E}-03$ & $1.75 \mathrm{E}-03$ & $3.49 \mathrm{E}-06$ & $1.02 \mathrm{E}-22$ & $0.00 \mathrm{E}+00$ & $0.00 \mathrm{E}+00$ & $0.00 \mathrm{E}+00$ & $0.00 \mathrm{E}+00$ & $0.00 \mathrm{E}+00$ & $0.00 \mathrm{E}+00$ \\
\hline SI 32 & $4.05 \mathrm{E}-13$ & $4.05 \mathrm{E}-13$ & $4.05 \mathrm{E}-13$ & $4.05 \mathrm{E}-13$ & $4.05 \mathrm{E}-13$ & 4.05E-13 & $4.05 \mathrm{E}-13$ & $4.05 \mathrm{E}-13$ & $4.05 \mathrm{E}-13$ & $4.04 \mathrm{E}-13$ \\
\hline P 32 & $3.47 \mathrm{E}-03$ & $3.47 \mathrm{E}-03$ & $3.31 \mathrm{E}-03$ & $2.47 \mathrm{E}-03$ & $8.10 \mathrm{E}-04$ & 1.89E-04 & $4.42 \mathrm{E}-05$ & $1.03 E-05$ & $2.14 \mathrm{E}-07$ & $9.20 \mathrm{E}-11$ \\
\hline P 33 & $1.16 \mathrm{E}-07$ & $1.16 \mathrm{E}-07$ & $1.13 \mathrm{E}-07$ & $9.59 \mathrm{E}-08$ & 5.07E-08 & $2.21 \mathrm{E}-08$ & $9.60 \mathrm{E}-09$ & $4.18 \mathrm{E}-09$ & $4.55 \mathrm{E}-10$ & $5.39 \mathrm{E}-12$ \\
\hline P 34 & $6.53 \mathrm{E}-07$ & $0.00 \mathrm{E}+00$ & $0.00 \mathrm{E}+00$ & $0.00 \mathrm{E}+00$ & $0.00 \mathrm{E}+00$ & $0.00 \mathrm{E}+00$ & $0.00 \mathrm{E}+00$ & $0.00 \mathrm{E}+00$ & $0.00 \mathrm{E}+00$ & $0.00 \mathrm{E}+00$ \\
\hline S 35 & $6.15 \mathrm{E}-05$ & $6.15 \mathrm{E}-05$ & $6.10 \mathrm{E}-05$ & $5.82 \mathrm{E}-05$ & $4.86 \mathrm{E}-05$ & $3.83 \mathrm{E}-05$ & $3.03 \mathrm{E}-05$ & $2.39 \mathrm{E}-05$ & $1.27 \mathrm{E}-05$ & $3.61 \mathrm{E}-06$ \\
\hline S 37 & $1.89 \mathrm{E}-07$ & $3.10 \mathrm{E}-09$ & $0.00 \mathrm{E}+00$ & $0.00 \mathrm{E}+00$ & $0.00 \mathrm{E}+00$ & $0.00 \mathrm{E}+00$ & $0.00 \mathrm{E}+00$ & $0.00 \mathrm{E}+00$ & $0.00 \mathrm{E}+00$ & $0.00 \mathrm{E}+00$ \\
\hline CL 36 & $7.84 \mathrm{E}-13$ & $7.84 \mathrm{E}-13$ & $7.84 \mathrm{E}-13$ & $7.84 \mathrm{E}-13$ & $7.84 \mathrm{E}-13$ & $7.84 \mathrm{E}-13$ & $7.84 \mathrm{E}-13$ & $7.84 \mathrm{E}-13$ & $7.84 \mathrm{E}-13$ & $7.84 \mathrm{E}-13$ \\
\hline $\mathrm{CL} 38$ & $8.04 \mathrm{E}-11$ & $4.60 \mathrm{E}-11$ & $1.81 \mathrm{E}-22$ & $0.00 \mathrm{E}+00$ & $0.00 \mathrm{E}+00$ & $0.00 \mathrm{E}+00$ & $0.00 \mathrm{E}+00$ & $0.00 \mathrm{E}+00$ & $0.00 \mathrm{E}+00$ & $0.00 \mathrm{E}+00$ \\
\hline CL 38M & $9.57 \mathrm{E}-13$ & $0.00 \mathrm{E}+00$ & $0.00 \mathrm{E}+00$ & $0.00 \mathrm{E}+00$ & $0.00 \mathrm{E}+00$ & $0.00 \mathrm{E}+00$ & $0.00 \mathrm{E}+00$ & $0.00 \mathrm{E}+00$ & $0.00 \mathrm{E}+00$ & $0.00 \mathrm{E}+00$ \\
\hline CA 45 & $5.16 \mathrm{E}-13$ & $5.16 \mathrm{E}-13$ & $5.14 \mathrm{E}-13$ & $5.01 \mathrm{E}-13$ & $4.54 \mathrm{E}-13$ & $4.00 \mathrm{E}-13$ & $3.52 \mathrm{E}-13$ & $3.10 \mathrm{E}-13$ & $2.21 \mathrm{E}-13$ & $1.12 \mathrm{E}-13$ \\
\hline CA 47 & $3.65 \mathrm{E}-12$ & $3.64 \mathrm{E}-12$ & $3.13 \mathrm{E}-12$ & $1.25 \mathrm{E}-12$ & $3.72 \mathrm{E}-14$ & $3.81 \mathrm{E}-16$ & $5.76 \mathrm{E}-18$ & $2.01 \mathrm{E}-18$ & $9.86 \mathrm{E}-24$ & $0.00 \mathrm{E}+00$ \\
\hline SC 46 & $9.19 \mathrm{E}-16$ & $9.19 \mathrm{E}-16$ & $9.12 \mathrm{E}-16$ & $8.68 \mathrm{E}-16$ & 7.17E-16 & 5.59E-16 & $4.36 \mathrm{E}-16$ & $3.40 \mathrm{E}-16$ & $1.76 \mathrm{E}-16$ & 4.67E-17 \\
\hline SC 47 & $1.69 \mathrm{E}-08$ & $1.68 \mathrm{E}-08$ & $1.37 \mathrm{E}-08$ & $3.97 \mathrm{E}-09$ & $3.42 \mathrm{E}-11$ & $7.02 \mathrm{E}-14$ & $1.53 \mathrm{E}-16$ & $0.00 \mathrm{E}+00$ & $3.73 \mathrm{E}-23$ & $0.00 \mathrm{E}+00$ \\
\hline SC 48 & $1.66 \mathrm{E}-08$ & $1.65 \mathrm{E}-08$ & 1.14E-08 & 1.17E-09 & $1.88 \mathrm{E}-13$ & $2.11 \mathrm{E}-18$ & $2.38 \mathrm{E}-23$ & $2.69 \mathrm{E}-28$ & $0.00 \mathrm{E}+00$ & $0.00 \mathrm{E}+00$ \\
\hline SC 49 & $4.71 \mathrm{E}-09$ & $3.28 \mathrm{E}-09$ & $1.36 \mathrm{E}-16$ & $0.00 \mathrm{E}+00$ & $0.00 \mathrm{E}+00$ & $0.00 \mathrm{E}+00$ & $0.00 \mathrm{E}+00$ & $0.00 \mathrm{E}+00$ & $0.00 \mathrm{E}+00$ & $0.00 \mathrm{E}+00$ \\
\hline SC 50 & $4.02 \mathrm{E}-10$ & $2.08 \mathrm{E}-15$ & $0.00 \mathrm{E}+00$ & $0.00 \mathrm{E}+00$ & $0.00 \mathrm{E}+00$ & $0.00 \mathrm{E}+00$ & $0.00 \mathrm{E}+00$ & $0.00 \mathrm{E}+00$ & $0.00 \mathrm{E}+00$ & $0.00 \mathrm{E}+00$ \\
\hline TI 51 & $1.15 \mathrm{E}-05$ & $3.11 \mathrm{E}-07$ & $0.00 \mathrm{E}+00$ & $0.00 \mathrm{E}+00$ & $0.00 \mathrm{E}+00$ & $0.00 \mathrm{E}+00$ & $0.00 \mathrm{E}+00$ & $0.00 \mathrm{E}+00$ & $0.00 \mathrm{E}+00$ & $0.00 \mathrm{E}+00$ \\
\hline V 52 & $1.90 \mathrm{E}-02$ & 7.41E-05 & $0.00 \mathrm{E}+00$ & $0.00 \mathrm{E}+00$ & $0.00 \mathrm{E}+00$ & $0.00 \mathrm{E}+00$ & $0.00 \mathrm{E}+00$ & $0.00 \mathrm{E}+00$ & $0.00 \mathrm{E}+00$ & $0.00 \mathrm{E}+00$ \\
\hline V 53 & $8.70 \mathrm{E}-05$ & $2.14 \mathrm{E}-10$ & $0.00 \mathrm{E}+00$ & $0.00 \mathrm{E}+00$ & $0.00 \mathrm{E}+00$ & $0.00 \mathrm{E}+00$ & $0.00 \mathrm{E}+00$ & $0.00 \mathrm{E}+00$ & $0.00 \mathrm{E}+00$ & $0.00 \mathrm{E}+00$ \\
\hline V54 & $9.20 \mathrm{E}-07$ & $1.29 \mathrm{E}-16$ & $0.00 \mathrm{E}+00$ & $0.00 \mathrm{E}+00$ & $0.00 \mathrm{E}+00$ & $0.00 \mathrm{E}+00$ & $0.00 \mathrm{E}+00$ & $0.00 \mathrm{E}+00$ & $0.00 \mathrm{E}+00$ & $0.00 \mathrm{E}+00$ \\
\hline CR 51 & $1.88 \mathrm{E}+00$ & $1.88 \mathrm{E}+00$ & $1.83 \mathrm{E}+00$ & $1.58 \mathrm{E}+00$ & $8.88 \mathrm{E}-01$ & 4.19E-01 & $1.98 \mathrm{E}-01$ & $9.34 \mathrm{E}-02$ & $1.26 \mathrm{E}-02$ & $2.31 \mathrm{E}-04$ \\
\hline CR 55 & $2.63 \mathrm{E}-02$ & $7.52 \mathrm{E}-05$ & $0.00 \mathrm{E}+00$ & $0.00 \mathrm{E}+00$ & $0.00 \mathrm{E}+00$ & $0.00 \mathrm{E}+00$ & $0.00 \mathrm{E}+00$ & $0.00 \mathrm{E}+00$ & $0.00 \mathrm{E}+00$ & $0.00 \mathrm{E}+00$ \\
\hline MN 54 & $4.30 \mathrm{E}-02$ & $4.30 \mathrm{E}-02$ & $4.29 \mathrm{E}-02$ & $4.24 \mathrm{E}-02$ & $4.03 \mathrm{E}-02$ & $3.77 \mathrm{E}-02$ & $3.53 \mathrm{E}-02$ & $3.30 \mathrm{E}-02$ & $2.76 \mathrm{E}-02$ & $1.94 \mathrm{E}-02$ \\
\hline MN 56 & $4.84 \mathrm{E}+00$ & $4.23 \mathrm{E}+00$ & $7.65 \mathrm{E}-03$ & $1.18 \mathrm{E}-19$ & $0.00 \mathrm{E}+00$ & $0.00 \mathrm{E}+00$ & $0.00 \mathrm{E}+00$ & $0.00 \mathrm{E}+00$ & $0.00 \mathrm{E}+00$ & $0.00 \mathrm{E}+00$ \\
\hline MN 57 & 2.81E-04 & $6.91 \mathrm{E}-10$ & $0.00 \mathrm{E}+00$ & $0.00 \mathrm{E}+00$ & $0.00 \mathrm{E}+00$ & $0.00 \mathrm{E}+00$ & $0.00 \mathrm{E}+00$ & $0.00 \mathrm{E}+00$ & $0.00 \mathrm{E}+00$ & $0.00 \mathrm{E}+00$ \\
\hline MN 58 & $8.65 \mathrm{E}-07$ & $4.36 \mathrm{E}-15$ & $0.00 \mathrm{E}+00$ & $0.00 \mathrm{E}+00$ & $0.00 \mathrm{E}+00$ & $0.00 \mathrm{E}+00$ & $0.00 \mathrm{E}+00$ & $0.00 \mathrm{E}+00$ & $0.00 \mathrm{E}+00$ & $0.00 \mathrm{E}+00$ \\
\hline FE 55 & $1.76 \mathrm{E}-01$ & $1.76 \mathrm{E}-01$ & $1.76 \mathrm{E}-01$ & $1.75 \mathrm{E}-01$ & $1.72 \mathrm{E}-01$ & $1.69 \mathrm{E}-01$ & $1.65 \mathrm{E}-01$ & $1.61 \mathrm{E}-01$ & $1.52 \mathrm{E}-01$ & $1.35 \mathrm{E}-01$ \\
\hline FE 59 & $3.32 \mathrm{E}-02$ & $3.32 \mathrm{E}-02$ & $3.27 \mathrm{E}-02$ & $2.98 \mathrm{E}-02$ & $2.09 \mathrm{E}-02$ & $1.32 \mathrm{E}-02$ & $8.30 \mathrm{E}-03$ & $5.23 \mathrm{E}-03$ & $1.52 \mathrm{E}-03$ & $1.30 \mathrm{E}-04$ \\
\hline CO 58 & $1.16 \mathrm{E}-01$ & $1.16 \mathrm{E}-01$ & $1.15 \mathrm{E}-01$ & $1.09 \mathrm{E}-01$ & $8.68 \mathrm{E}-02$ & $6.47 \mathrm{E}-02$ & $4.82 \mathrm{E}-02$ & $3.59 \mathrm{E}-02$ & 1.64E-02 & $3.43 \mathrm{E}-03$ \\
\hline CO 60 & $5.11 \mathrm{E}-04$ & $5.11 \mathrm{E}-04$ & 5.11E-04 & $5.10 \mathrm{E}-04$ & $5.05 \mathrm{E}-04$ & $5.00 \mathrm{E}-04$ & 4.95E-04 & 4.89E-04 & $4.75 \mathrm{E}-04$ & 4.49E-04 \\
\hline $\mathrm{CO} 60 \mathrm{M}$ & $4.31 \mathrm{E}-03$ & $5.91 \mathrm{E}-04$ & $0.00 \mathrm{E}+00$ & $0.00 \mathrm{E}+00$ & $0.00 \mathrm{E}+00$ & $0.00 \mathrm{E}+00$ & $0.00 \mathrm{E}+00$ & $0.00 E+00$ & $0.00 \mathrm{E}+00$ & $0.00 \mathrm{E}+00$ \\
\hline CO 61 & $1.45 \mathrm{E}-04$ & $1.18 \mathrm{E}-04$ & $6.07 E-09$ & $0.00 \mathrm{E}+00$ & $0.00 \mathrm{E}+00$ & $0.00 \mathrm{E}+00$ & $0.00 \mathrm{E}+00$ & $0.00 \mathrm{E}+00$ & $0.00 \mathrm{E}+00$ & $0.00 \mathrm{E}+00$ \\
\hline CO 62 & $2.23 \mathrm{E}-05$ & $2.13 \mathrm{E}-11$ & $0.00 \mathrm{E}+00$ & $0.00 \mathrm{E}+00$ & $0.00 \mathrm{E}+00$ & $0.00 \mathrm{E}+00$ & $0.00 \mathrm{E}+00$ & $0.00 \mathrm{E}+00$ & $0.00 \mathrm{E}+00$ & $0.00 \mathrm{E}+00$ \\
\hline
\end{tabular}


TEM-10200-1

$12 / 19 / 17$

Rev.08

Title:

AS-RUN NEUTRONICS EVALUATION FOR THE GE HITACHI EXPERIMENT IN THE ATR

ECAR NO.: $4740 \quad$ REV. NO.: 0

\begin{tabular}{|c|c|c|c|c|c|c|c|c|c|c|}
\hline \multicolumn{11}{|c|}{$1 \mathrm{~g}$ of $316 \mathrm{SS}$} \\
\hline Ilenton & EOI & $30.0 \mathrm{MI}$ & $1.0 \mathrm{D}$ & $7.0 \mathrm{D}$ & $30.0 \mathrm{D}$ & $60.0 \mathrm{D}$ & $90.0 \mathrm{D}$ & $120.0 \mathrm{D}$ & $200.0 \mathrm{D}$ & $360.0 \mathrm{D}$ \\
\hline Isotope & $\mathrm{Ci} / 1 \mathrm{~g}$ of $316 \mathrm{SS}$ & $\mathrm{Ci} / 1 \mathrm{~g}$ of $316 \mathrm{SS}$ & $\mathrm{Ci} / 1 \mathrm{~g}$ of $316 \mathrm{SS}$ & $\mathrm{Ci} / 1 \mathrm{~g}$ of $316 \mathrm{SS}$ & $\mathrm{Ci} / 1 \mathrm{~g}$ of $316 \mathrm{SS}$ & $\mathrm{Ci} / 1 \mathrm{~g}$ of $316 \mathrm{SS}$ & $\mathrm{Ci} / 1 \mathrm{~g}$ of $316 \mathrm{SS}$ & $\mathrm{Ci} / 1 \mathrm{~g}$ of $316 \mathrm{SS}$ & $\mathrm{Ci} / 1 \mathrm{~g}$ of $316 \mathrm{SS}$ & $\mathrm{Ci} / 1 \mathrm{~g}$ of $316 \mathrm{SS}$ \\
\hline NI 59 & $3.33 \mathrm{E}-05$ & $3.33 \mathrm{E}-05$ & $3.33 \mathrm{E}-05$ & $3.33 \mathrm{E}-05$ & $3.33 \mathrm{E}-05$ & $3.33 \mathrm{E}-05$ & $3.33 \mathrm{E}-05$ & $3.33 \mathrm{E}-05$ & $3.33 \mathrm{E}-05$ & $3.33 \mathrm{E}-05$ \\
\hline NI 63 & $4.30 \mathrm{E}-03$ & $4.30 \mathrm{E}-03$ & $4.30 \mathrm{E}-03$ & $4.30 \mathrm{E}-03$ & $4.30 \mathrm{E}-03$ & $4.30 \mathrm{E}-03$ & $4.29 \mathrm{E}-03$ & $4.29 \mathrm{E}-03$ & $4.28 \mathrm{E}-03$ & $4.27 \mathrm{E}-03$ \\
\hline NI 65 & $2.75 \mathrm{E}-02$ & $2.40 \mathrm{E}-02$ & $3.75 \mathrm{E}-05$ & $2.36 \mathrm{E}-22$ & $0.00 \mathrm{E}+00$ & $0.00 \mathrm{E}+00$ & $0.00 \mathrm{E}+00$ & $0.00 \mathrm{E}+00$ & $0.00 \mathrm{E}+00$ & $0.00 \mathrm{E}+00$ \\
\hline NI 66 & $4.20 \mathrm{E}-07$ & $4.17 \mathrm{E}-07$ & $3.10 \mathrm{E}-07$ & $4.98 \mathrm{E}-08$ & $4.51 \mathrm{E}-11$ & $4.85 \mathrm{E}-15$ & $5.21 \mathrm{E}-19$ & $5.59 \mathrm{E}-23$ & $0.00 \mathrm{E}+00$ & $0.00 \mathrm{E}+00$ \\
\hline CU 64 & $1.06 \mathrm{E}-05$ & $1.03 \mathrm{E}-05$ & $2.85 \mathrm{E}-06$ & $1.10 \mathrm{E}-09$ & $9.06 \mathrm{E}-23$ & $0.00 \mathrm{E}+00$ & $0.00 \mathrm{E}+00$ & $0.00 \mathrm{E}+00$ & $0.00 \mathrm{E}+00$ & $0.00 \mathrm{E}+00$ \\
\hline CU 66 & $6.50 \mathrm{E}-05$ & $1.51 \mathrm{E}-06$ & $3.10 \mathrm{E}-07$ & $4.98 \mathrm{E}-08$ & $4.52 \mathrm{E}-11$ & $4.85 \mathrm{E}-15$ & $5.21 \mathrm{E}-19$ & $5.60 \mathrm{E}-23$ & $0.00 \mathrm{E}+00$ & $0.00 \mathrm{E}+00$ \\
\hline CU 67 & $1.86 \mathrm{E}-10$ & $1.85 \mathrm{E}-10$ & $1.42 \mathrm{E}-10$ & $2.83 \mathrm{E}-11$ & $5.82 \mathrm{E}-14$ & $1.82 \mathrm{E}-17$ & $5.72 \mathrm{E}-21$ & $1.79 \mathrm{E}-24$ & $0.00 \mathrm{E}+00$ & $0.00 \mathrm{E}+00$ \\
\hline $\mathrm{ZN} 65$ & $1.79 E-10$ & $1.79 \mathrm{E}-10$ & $1.78 \mathrm{E}-10$ & $1.75 \mathrm{E}-10$ & $1.64 \mathrm{E}-10$ & $1.51 \mathrm{E}-10$ & $1.38 \mathrm{E}-10$ & $1.27 \mathrm{E}-10$ & $1.01 \mathrm{E}-10$ & $6.42 \mathrm{E}-11$ \\
\hline ZN 69 & $4.37 \mathrm{E}-14$ & $3.15 \mathrm{E}-14$ & $9.41 \mathrm{E}-16$ & $6.59 \mathrm{E}-19$ & $0.00 \mathrm{E}+00$ & $0.00 \mathrm{E}+00$ & $0.00 \mathrm{E}+00$ & $0.00 \mathrm{E}+00$ & $0.00 \mathrm{E}+00$ & $0.00 \mathrm{E}+00$ \\
\hline SR 89 & $1.81 \mathrm{E}-10$ & $1.81 \mathrm{E}-10$ & $1.78 \mathrm{E}-10$ & $1.64 \mathrm{E}-10$ & $1.20 \mathrm{E}-10$ & $7.93 \mathrm{E}-11$ & $5.25 \mathrm{E}-11$ & $3.48 \mathrm{E}-11$ & $1.16 \mathrm{E}-11$ & $1.29 \mathrm{E}-12$ \\
\hline SR 90 & $4.58 \mathrm{E}-14$ & $4.58 \mathrm{E}-14$ & $4.58 \mathrm{E}-14$ & $4.58 \mathrm{E}-14$ & $4.57 \mathrm{E}-14$ & $4.56 \mathrm{E}-14$ & $4.55 \mathrm{E}-14$ & $4.54 \mathrm{E}-14$ & $4.52 \mathrm{E}-14$ & 4.47E-14 \\
\hline SR 91 & $4.44 \mathrm{E}-12$ & $4.28 \mathrm{E}-12$ & $7.71 \mathrm{E}-13$ & $2.11 \mathrm{E}-17$ & $0.00 \mathrm{E}+00$ & $0.00 \mathrm{E}+00$ & $0.00 \mathrm{E}+00$ & $0.00 \mathrm{E}+00$ & $0.00 \mathrm{E}+00$ & $0.00 \mathrm{E}+00$ \\
\hline Y 89M & $3.79 \mathrm{E}-06$ & $3.78 \mathrm{E}-06$ & $3.07 E-06$ & $8.59 \mathrm{E}-07$ & $6.54 \mathrm{E}-09$ & $1.13 \mathrm{E}-11$ & $1.95 \mathrm{E}-14$ & $3.54 \mathrm{E}-17$ & $1.52 \mathrm{E}-24$ & $0.00 \mathrm{E}+00$ \\
\hline Y 90 & 4.89E-09 & 4.86E-09 & $3.77 \mathrm{E}-09$ & $7.92 \mathrm{E}-10$ & $2.05 \mathrm{E}-12$ & $4.64 \mathrm{E}-14$ & $4.55 \mathrm{E}-14$ & $4.54 \mathrm{E}-14$ & $4.52 \mathrm{E}-14$ & 4.47E-14 \\
\hline Y 90M & $3.72 \mathrm{E}-12$ & $3.33 \mathrm{E}-12$ & $1.75 \mathrm{E}-14$ & $1.82 \mathrm{E}-28$ & $0.00 \mathrm{E}+00$ & $0.00 \mathrm{E}+00$ & $0.00 \mathrm{E}+00$ & $0.00 \mathrm{E}+00$ & $0.00 \mathrm{E}+00$ & $0.00 \mathrm{E}+00$ \\
\hline Y 91 & 2.93E-11 & 2.93E-11 & $2.90 \mathrm{E}-11$ & $2.70 \mathrm{E}-11$ & $2.06 \mathrm{E}-11$ & 1.44E-11 & $1.01 \mathrm{E}-11$ & $7.08 \mathrm{E}-12$ & $2.74 \mathrm{E}-12$ & $4.12 \mathrm{E}-13$ \\
\hline Y92 & $5.80 \mathrm{E}-10$ & $5.26 \mathrm{E}-10$ & $5.27 \mathrm{E}-12$ & $2.97 \mathrm{E}-24$ & $0.00 \mathrm{E}+00$ & $0.00 \mathrm{E}+00$ & $0.00 \mathrm{E}+00$ & $0.00 \mathrm{E}+00$ & $0.00 \mathrm{E}+00$ & $0.00 \mathrm{E}+00$ \\
\hline Y 94 & $3.17 E-12$ & 1.07E-12 & $0.00 \mathrm{E}+00$ & $0.00 \mathrm{E}+00$ & $0.00 \mathrm{E}+00$ & $0.00 \mathrm{E}+00$ & $0.00 \mathrm{E}+00$ & $0.00 \mathrm{E}+00$ & $0.00 E+00$ & $0.00 \mathrm{E}+00$ \\
\hline ZR 89 & $3.80 \mathrm{E}-06$ & $3.78 \mathrm{E}-06$ & $3.07 E-06$ & $8.61 \mathrm{E}-07$ & $6.55 \mathrm{E}-09$ & $1.13 \mathrm{E}-11$ & $1.95 \mathrm{E}-14$ & $3.55 \mathrm{E}-17$ & $1.52 \mathrm{E}-24$ & $0.00 \mathrm{E}+00$ \\
\hline ZR 93 & $1.14 \mathrm{E}-11$ & 1.14E-11 & $1.14 \mathrm{E}-11$ & 1.14E-11 & $1.14 \mathrm{E}-11$ & $1.14 \mathrm{E}-11$ & $1.14 \mathrm{E}-11$ & 1.14E-11 & $1.14 \mathrm{E}-11$ & $1.14 \mathrm{E}-11$ \\
\hline ZR 95 & $1.19 \mathrm{E}-05$ & 1.19E-05 & $1.18 \mathrm{E}-05$ & $1.10 \mathrm{E}-05$ & $8.60 \mathrm{E}-06$ & $6.21 \mathrm{E}-06$ & 4.49E-06 & $3.24 \mathrm{E}-06$ & $1.36 \mathrm{E}-06$ & $2.41 \mathrm{E}-07$ \\
\hline ZR 97 & $3.00 \mathrm{E}-07$ & 2.94E-07 & $1.12 \mathrm{E}-07$ & $3.06 \mathrm{E}-10$ & $4.50 \mathrm{E}-20$ & $0.00 \mathrm{E}+00$ & $0.00 \mathrm{E}+00$ & $0.00 \mathrm{E}+00$ & $0.00 \mathrm{E}+00$ & $0.00 \mathrm{E}+00$ \\
\hline NB 92 & $7.56 \mathrm{E}-04$ & $7.55 \mathrm{E}-04$ & $7.06 \mathrm{E}-04$ & 4.69E-04 & $9.76 \mathrm{E}-05$ & $1.26 \mathrm{E}-05$ & $1.63 \mathrm{E}-06$ & $2.10 \mathrm{E}-07$ & $8.97 \mathrm{E}-10$ & $1.63 \mathrm{E}-14$ \\
\hline NB 93M & $1.64 \mathrm{E}-13$ & 1.64E-13 & $1.65 \mathrm{E}-13$ & $1.74 \mathrm{E}-13$ & $2.08 \mathrm{E}-13$ & $2.53 \mathrm{E}-13$ & $2.97 \mathrm{E}-13$ & $3.41 \mathrm{E}-13$ & $4.58 \mathrm{E}-13$ & $6.88 \mathrm{E}-13$ \\
\hline NB 94 & $1.28 \mathrm{E}-09$ & $1.28 \mathrm{E}-09$ & $1.28 \mathrm{E}-09$ & $1.28 \mathrm{E}-09$ & $1.28 \mathrm{E}-09$ & $1.28 \mathrm{E}-09$ & $1.28 \mathrm{E}-09$ & $1.28 \mathrm{E}-09$ & $1.28 \mathrm{E}-09$ & $1.28 \mathrm{E}-09$ \\
\hline NB 95 & $2.89 \mathrm{E}-05$ & $2.89 \mathrm{E}-05$ & $2.86 \mathrm{E}-05$ & 2.67E-05 & $2.05 E-05$ & $1.46 \mathrm{E}-05$ & $1.04 \mathrm{E}-05$ & 7.43E-06 & 3.07E-06 & $5.36 \mathrm{E}-07$ \\
\hline NB 95M & $8.28 \mathrm{E}-08$ & $8.28 \mathrm{E}-08$ & $8.28 \mathrm{E}-08$ & 8.04E-08 & $6.37 \mathrm{E}-08$ & $4.61 \mathrm{E}-08$ & $3.33 \mathrm{E}-08$ & $2.41 \mathrm{E}-08$ & $1.01 \mathrm{E}-08$ & 1.79E-09 \\
\hline NB 96 & $2.71 \mathrm{E}-05$ & $2.67 \mathrm{E}-05$ & $1.33 \mathrm{E}-05$ & 1.85E-07 & $1.41 \mathrm{E}-14$ & $7.37 E-24$ & $0.00 \mathrm{E}+00$ & $0.00 E+00$ & $0.00 E+00$ & $0.00 \mathrm{E}+00$ \\
\hline NB 97 & $1.06 E-05$ & $8.05 \mathrm{E}-06$ & $1.13 E-07$ & $3.08 \mathrm{E}-10$ & $4.85 \mathrm{E}-20$ & $0.00 \mathrm{E}+00$ & $0.00 \mathrm{E}+00$ & $0.00 \mathrm{E}+00$ & $0.00 \mathrm{E}+00$ & $0.00 \mathrm{E}+00$ \\
\hline NB 97M & $2.84 \mathrm{E}-07$ & $2.79 \mathrm{E}-07$ & $1.06 \mathrm{E}-07$ & $2.90 \mathrm{E}-10$ & $4.26 \mathrm{E}-20$ & $0.00 \mathrm{E}+00$ & $0.00 \mathrm{E}+00$ & $0.00 \mathrm{E}+00$ & $0.00 \mathrm{E}+00$ & $0.00 \mathrm{E}+00$ \\
\hline NB 98 & $1.59 \mathrm{E}-06$ & $0.00 \mathrm{E}+00$ & $0.00 \mathrm{E}+00$ & $0.00 \mathrm{E}+00$ & $0.00 \mathrm{E}+00$ & $0.00 \mathrm{E}+00$ & $0.00 \mathrm{E}+00$ & $0.00 \mathrm{E}+00$ & $0.00 \mathrm{E}+00$ & $0.00 \mathrm{E}+00$ \\
\hline NB100 & $7.89 E-08$ & $0.00 \mathrm{E}+00$ & $0.00 \mathrm{E}+00$ & $0.00 \mathrm{E}+00$ & $0.00 \mathrm{E}+00$ & $0.00 \mathrm{E}+00$ & $0.00 \mathrm{E}+00$ & $0.00 \mathrm{E}+00$ & $0.00 \mathrm{E}+00$ & $0.00 \mathrm{E}+00$ \\
\hline MO 93M & $2.20 \mathrm{E}-04$ & 2.09E-04 & $1.94 \mathrm{E}-05$ & 9.09E-12 & $0.00 \mathrm{E}+00$ & $0.00 E+00$ & $0.00 \mathrm{E}+00$ & $0.00 \mathrm{E}+00$ & $0.00 \mathrm{E}+00$ & $0.00 \mathrm{E}+00$ \\
\hline MO 93 & $8.04 \mathrm{E}-07$ & $8.04 \mathrm{E}-07$ & $8.04 \mathrm{E}-07$ & $8.04 \mathrm{E}-07$ & $8.04 \mathrm{E}-07$ & $8.04 \mathrm{E}-07$ & $8.04 \mathrm{E}-07$ & $8.04 \mathrm{E}-07$ & $8.04 \mathrm{E}-07$ & $8.04 \mathrm{E}-07$ \\
\hline MO 99 & $1.54 \mathrm{E}-01$ & $1.53 \mathrm{E}-01$ & 1.19E-01 & $2.63 \mathrm{E}-02$ & 7.99E-05 & $4.15 \mathrm{E}-08$ & $2.16 \mathrm{E}-11$ & $1.12 \mathrm{E}-14$ & $1.97 E-23$ & $0.00 \mathrm{E}+00$ \\
\hline MO101 & $3.82 \mathrm{E}-02$ & $9.22 \mathrm{E}-03$ & $0.00 \mathrm{E}+00$ & $0.00 \mathrm{E}+00$ & $0.00 \mathrm{E}+00$ & $0.00 \mathrm{E}+00$ & $0.00 \mathrm{E}+00$ & $0.00 \mathrm{E}+00$ & $0.00 \mathrm{E}+00$ & $0.00 \mathrm{E}+00$ \\
\hline 99 & $3.51 \mathrm{E}-08$ & $3.51 \mathrm{E}-08$ & 3.53 & 3.57 & 3.58 & 3.58 & $3.58 \mathrm{E}-08$ & $3.58 \mathrm{E}-08$ & -08 & 08 \\
\hline
\end{tabular}


TEM-10200-1

$12 / 19 / 17$

Rev.08

Title:

AS-RUN NEUTRONICS EVALUATION FOR THE GE HITACHI EXPERIMENT IN THE ATR

ECAR NO:

\section{ENGINEERING CALCULATIONS AND ANALYSIS}

PROJECT NO.: 32535 Date: $12 / 02 / 2019$

\begin{tabular}{|c|c|c|c|c|c|c|c|c|c|c|}
\hline \multicolumn{11}{|c|}{$1 \mathrm{~g}$ of $316 \mathrm{SS}$} \\
\hline Isotone & EOI & $30.0 \mathrm{MI}$ & $1.0 \mathrm{D}$ & $7.0 \mathrm{D}$ & $30.0 \mathrm{D}$ & $60.0 \mathrm{D}$ & $90.0 \mathrm{D}$ & $120.0 \mathrm{D}$ & $200.0 \mathrm{D}$ & $360.0 \mathrm{D}$ \\
\hline sotope & $\mathrm{Ci} / 1 \mathrm{~g}$ of $316 \mathrm{SS}$ & $\mathrm{Ci} / 1 \mathrm{~g}$ of $316 \mathrm{SS}$ & $\mathrm{Ci} / 1 \mathrm{~g}$ of $316 \mathrm{SS}$ & $\mathrm{Ci} / 1 \mathrm{~g}$ of $316 \mathrm{SS}$ & $\mathrm{Ci} / 1 \mathrm{~g}$ of $316 \mathrm{SS}$ & $\mathrm{Ci} / 1 \mathrm{~g}$ of $316 \mathrm{SS}$ & $\mathrm{Ci} / 1 \mathrm{~g}$ of $316 \mathrm{SS}$ & $\mathrm{Ci} / 1 \mathrm{~g}$ of $316 \mathrm{SS}$ & $\mathrm{Ci} / 1 \mathrm{~g}$ of $316 \mathrm{SS}$ & $\mathrm{Ci} / 1 \mathrm{~g}$ of $316 \mathrm{SS}$ \\
\hline TC100 & $1.64 \mathrm{E}-03$ & $0.00 \mathrm{E}+00$ & $0.00 \mathrm{E}+00$ & $0.00 \mathrm{E}+00$ & $0.00 \mathrm{E}+00$ & $0.00 \mathrm{E}+00$ & $0.00 \mathrm{E}+00$ & $0.00 \mathrm{E}+00$ & $0.00 \mathrm{E}+00$ & $0.00 \mathrm{E}+00$ \\
\hline TC101 & $3.82 \mathrm{E}-02$ & $2.21 \mathrm{E}-02$ & $0.00 \mathrm{E}+00$ & $0.00 \mathrm{E}+00$ & $0.00 \mathrm{E}+00$ & $0.00 \mathrm{E}+00$ & $0.00 \mathrm{E}+00$ & $0.00 \mathrm{E}+00$ & $0.00 \mathrm{E}+00$ & $0.00 \mathrm{E}+00$ \\
\hline RU103 & $8.99 \mathrm{E}-07$ & $8.99 \mathrm{E}-07$ & $8.83 \mathrm{E}-07$ & $7.95 \mathrm{E}-07$ & $5.30 \mathrm{E}-07$ & $3.12 \mathrm{E}-07$ & $1.84 \mathrm{E}-07$ & $1.08 \mathrm{E}-07$ & $2.64 \mathrm{E}-08$ & 1.57E-09 \\
\hline RU105 & $4.67 \mathrm{E}-12$ & $4.32 \mathrm{E}-12$ & $1.10 \mathrm{E}-13$ & $1.89 \mathrm{E}-23$ & $0.00 \mathrm{E}+00$ & $0.00 \mathrm{E}+00$ & $0.00 \mathrm{E}+00$ & $0.00 \mathrm{E}+00$ & $0.00 \mathrm{E}+00$ & $0.00 \mathrm{E}+00$ \\
\hline RH104 & $9.31 \mathrm{E}-09$ & $6.68 \mathrm{E}-12$ & $0.00 \mathrm{E}+00$ & $0.00 \mathrm{E}+00$ & $0.00 \mathrm{E}+00$ & $0.00 \mathrm{E}+00$ & $0.00 \mathrm{E}+00$ & $0.00 \mathrm{E}+00$ & $0.00 \mathrm{E}+00$ & $0.00 \mathrm{E}+00$ \\
\hline RH104M & $6.74 \mathrm{E}-10$ & $5.60 \mathrm{E}-12$ & $0.00 \mathrm{E}+00$ & $0.00 \mathrm{E}+00$ & $0.00 \mathrm{E}+00$ & $0.00 \mathrm{E}+00$ & $0.00 \mathrm{E}+00$ & $0.00 \mathrm{E}+00$ & $0.00 \mathrm{E}+00$ & $0.00 \mathrm{E}+00$ \\
\hline RH105 & $3.82 \mathrm{E}-12$ & $3.83 \mathrm{E}-12$ & $2.79 \mathrm{E}-12$ & $1.67 \mathrm{E}-13$ & $3.33 \mathrm{E}-18$ & $2.47 \mathrm{E}-24$ & $1.84 \mathrm{E}-30$ & $0.00 \mathrm{E}+00$ & $0.00 \mathrm{E}+00$ & $0.00 \mathrm{E}+00$ \\
\hline RH105M & $1.31 \mathrm{E}-12$ & $1.21 \mathrm{E}-12$ & $3.09 \mathrm{E}-14$ & $5.30 \mathrm{E}-24$ & $0.00 \mathrm{E}+00$ & $0.00 \mathrm{E}+00$ & $0.00 \mathrm{E}+00$ & $0.00 \mathrm{E}+00$ & $0.00 \mathrm{E}+00$ & $0.00 \mathrm{E}+00$ \\
\hline RH106 & $3.77 \mathrm{E}-13$ & $1.28 \mathrm{E}-18$ & $1.28 \mathrm{E}-18$ & $1.28 \mathrm{E}-18$ & $1.23 \mathrm{E}-18$ & $1.16 \mathrm{E}-18$ & 1.09E-18 & 1.03E-18 & $8.86 \mathrm{E}-19$ & $6.51 \mathrm{E}-19$ \\
\hline RH106M & $1.84 \mathrm{E}-13$ & $1.57 \mathrm{E}-13$ & $9.57 \mathrm{E}-17$ & $0.00 \mathrm{E}+00$ & $0.00 \mathrm{E}+00$ & $0.00 \mathrm{E}+00$ & $0.00 \mathrm{E}+00$ & $0.00 \mathrm{E}+00$ & $0.00 \mathrm{E}+00$ & $0.00 \mathrm{E}+00$ \\
\hline SUMTOT & $7.41 \mathrm{E}+00$ & $6.70 \mathrm{E}+00$ & $2.34 \mathrm{E}+00$ & $1.97 \mathrm{E}+00$ & $1.21 \mathrm{E}+00$ & $7.08 \mathrm{E}-01$ & $4.60 \mathrm{E}-01$ & $3.34 \mathrm{E}-01$ & $2.15 \mathrm{E}-01$ & $1.63 \mathrm{E}-01$ \\
\hline OTOTAL & $7.41 \mathrm{E}+00$ & $6.70 \mathrm{E}+00$ & $2.34 \mathrm{E}+00$ & $1.97 \mathrm{E}+00$ & $1.21 \mathrm{E}+00$ & $7.08 \mathrm{E}-01$ & 4.60E-01 & $3.34 \mathrm{E}-01$ & $2.15 \mathrm{E}-01$ & $1.63 \mathrm{E}-01$ \\
\hline
\end{tabular}

Table 20. $\mathrm{g} / 1 \mathrm{~g}$ of $316 \mathrm{SS}$

\begin{tabular}{|c|c|c|c|c|c|c|c|c|c|c|}
\hline \multicolumn{11}{|c|}{$1 \mathrm{~g}$ of $316 \mathrm{SS}$} \\
\hline & EOI & $30.0 \mathrm{MI}$ & $1.0 \mathrm{D}$ & $7.0 \mathrm{D}$ & $30.0 \mathrm{D}$ & $60.0 \mathrm{D}$ & $90.0 \mathrm{D}$ & $120.0 \mathrm{D}$ & $200.0 \mathrm{D}$ & $360.0 \mathrm{D}$ \\
\hline Isotope & $\mathrm{g} / 1 \mathrm{~g}$ of $316 \mathrm{SS}$ & $\mathrm{g} / 1 \mathrm{~g}$ of $316 \mathrm{SS}$ & $\mathrm{g} / 1 \mathrm{~g}$ of $316 \mathrm{SS}$ & $\mathrm{g} / 1 \mathrm{~g}$ of $316 \mathrm{SS}$ & $\mathrm{g} / 1 \mathrm{~g}$ of $316 \mathrm{SS}$ & $\mathrm{g} / 1 \mathrm{~g}$ of $316 \mathrm{SS}$ & $\mathrm{g} / 1 \mathrm{~g}$ of $316 \mathrm{SS}$ & $\mathrm{g} / 1 \mathrm{~g}$ of $316 \mathrm{SS}$ & $\mathrm{g} / 1 \mathrm{~g}$ of $316 \mathrm{SS}$ & $\mathrm{g} / 1 \mathrm{~g}$ of $316 \mathrm{SS}$ \\
\hline H 1 & $8.08 \mathrm{E}-07$ & $8.08 \mathrm{E}-07$ & $8.08 \mathrm{E}-07$ & $8.08 \mathrm{E}-07$ & $8.08 \mathrm{E}-07$ & $8.08 \mathrm{E}-07$ & $8.08 \mathrm{E}-07$ & $8.08 \mathrm{E}-07$ & $8.08 \mathrm{E}-07$ & $8.08 \mathrm{E}-07$ \\
\hline $\mathrm{H} 2$ & $2.82 \mathrm{E}-10$ & $2.82 \mathrm{E}-10$ & $2.82 \mathrm{E}-10$ & $2.82 \mathrm{E}-10$ & $2.82 \mathrm{E}-10$ & $2.82 \mathrm{E}-10$ & $2.82 \mathrm{E}-10$ & $2.82 \mathrm{E}-10$ & $2.82 \mathrm{E}-10$ & $2.82 \mathrm{E}-10$ \\
\hline H 3 & $1.26 \mathrm{E}-14$ & $1.26 \mathrm{E}-14$ & $1.26 \mathrm{E}-14$ & $1.26 \mathrm{E}-14$ & $1.26 \mathrm{E}-14$ & $1.25 \mathrm{E}-14$ & $1.24 \mathrm{E}-14$ & $1.24 \mathrm{E}-14$ & $1.22 \mathrm{E}-14$ & $1.19 \mathrm{E}-14$ \\
\hline HE 4 & $4.36 \mathrm{E}-07$ & $4.36 \mathrm{E}-07$ & $4.36 \mathrm{E}-07$ & $4.36 \mathrm{E}-07$ & $4.36 \mathrm{E}-07$ & $4.36 \mathrm{E}-07$ & $4.36 \mathrm{E}-07$ & $4.36 \mathrm{E}-07$ & $4.36 \mathrm{E}-07$ & $4.36 \mathrm{E}-07$ \\
\hline LI 6 & $8.54 \mathrm{E}-14$ & $8.54 \mathrm{E}-14$ & $8.54 \mathrm{E}-14$ & $8.54 \mathrm{E}-14$ & $8.54 \mathrm{E}-14$ & $8.54 \mathrm{E}-14$ & $8.54 \mathrm{E}-14$ & $8.54 \mathrm{E}-14$ & $8.54 \mathrm{E}-14$ & $8.54 \mathrm{E}-14$ \\
\hline BE 9 & $8.66 \mathrm{E}-10$ & $8.66 \mathrm{E}-10$ & $8.66 \mathrm{E}-10$ & $8.66 \mathrm{E}-10$ & $8.66 \mathrm{E}-10$ & $8.66 \mathrm{E}-10$ & $8.66 \mathrm{E}-10$ & $8.66 \mathrm{E}-10$ & $8.66 \mathrm{E}-10$ & $8.66 \mathrm{E}-10$ \\
\hline BE 10 & $2.21 \mathrm{E}-11$ & $2.21 \mathrm{E}-11$ & $2.21 \mathrm{E}-11$ & $2.21 \mathrm{E}-11$ & $2.21 \mathrm{E}-11$ & $2.21 \mathrm{E}-11$ & $2.21 \mathrm{E}-11$ & $2.21 \mathrm{E}-11$ & $2.21 \mathrm{E}-11$ & $2.21 \mathrm{E}-11$ \\
\hline B 11 & $2.06 \mathrm{E}-07$ & $2.06 \mathrm{E}-07$ & $2.06 \mathrm{E}-07$ & $2.06 \mathrm{E}-07$ & $2.06 \mathrm{E}-07$ & $2.06 \mathrm{E}-07$ & $2.06 \mathrm{E}-07$ & $2.06 \mathrm{E}-07$ & $2.06 \mathrm{E}-07$ & $2.06 \mathrm{E}-07$ \\
\hline C 12 & $2.96 \mathrm{E}-04$ & $2.96 \mathrm{E}-04$ & $2.96 \mathrm{E}-04$ & $2.96 \mathrm{E}-04$ & $2.96 \mathrm{E}-04$ & $2.96 \mathrm{E}-04$ & $2.96 \mathrm{E}-04$ & $2.96 \mathrm{E}-04$ & $2.96 \mathrm{E}-04$ & $2.96 \mathrm{E}-04$ \\
\hline C 13 & $3.61 \mathrm{E}-06$ & $3.61 \mathrm{E}-06$ & $3.61 \mathrm{E}-06$ & $3.61 \mathrm{E}-06$ & $3.61 \mathrm{E}-06$ & $3.61 \mathrm{E}-06$ & $3.61 \mathrm{E}-06$ & $3.61 \mathrm{E}-06$ & $3.61 \mathrm{E}-06$ & $3.61 \mathrm{E}-06$ \\
\hline C 14 & $1.81 \mathrm{E}-06$ & $1.81 \mathrm{E}-06$ & $1.81 \mathrm{E}-06$ & $1.81 \mathrm{E}-06$ & $1.81 \mathrm{E}-06$ & $1.81 \mathrm{E}-06$ & $1.81 \mathrm{E}-06$ & $1.81 \mathrm{E}-06$ & $1.81 \mathrm{E}-06$ & $1.81 \mathrm{E}-06$ \\
\hline N 14 & $9.94 \mathrm{E}-04$ & $9.94 \mathrm{E}-04$ & $9.94 \mathrm{E}-04$ & $9.94 \mathrm{E}-04$ & $9.94 \mathrm{E}-04$ & $9.94 \mathrm{E}-04$ & $9.94 \mathrm{E}-04$ & $9.94 \mathrm{E}-04$ & $9.94 \mathrm{E}-04$ & $9.94 \mathrm{E}-04$ \\
\hline
\end{tabular}


TEM-10200-1

$12 / 19 / 17$

Rev.08

Title: AS-RUN NEUTRONICS EVALUATION FOR THE GE HITACHI EXPERIMENT IN THE ATR \begin{tabular}{llllllll} 
ECAR NO.: & 4740 & REV. NO.: & 0 & PROJECT NO.: & 32535 & Date: & 12/02/2019 \\
\hline
\end{tabular}

\begin{tabular}{|c|c|c|c|c|c|c|c|c|c|c|}
\hline \multicolumn{11}{|c|}{$1 \mathrm{~g}$ of $316 \mathrm{SS}$} \\
\hline Icton & EOI & $30.0 \mathrm{Ml}$ & $1.0 \mathrm{D}$ & $7.0 \mathrm{D}$ & $30.0 \mathrm{D}$ & $60.0 \mathrm{D}$ & $90.0 \mathrm{D}$ & $120.0 \mathrm{D}$ & $200.0 \mathrm{D}$ & $360.0 \mathrm{D}$ \\
\hline Isotope & $\mathrm{g} / 1 \mathrm{~g}$ of $316 \mathrm{SS}$ & $\mathrm{g} / 1 \mathrm{~g}$ of $316 \mathrm{SS}$ & $\mathrm{g} / 1 \mathrm{~g}$ of $316 \mathrm{SS}$ & $\mathrm{g} / 1 \mathrm{~g}$ of $316 \mathrm{SS}$ & $\mathrm{g} / 1 \mathrm{~g}$ of $316 \mathrm{SS}$ & $\mathrm{g} / 1 \mathrm{~g}$ of $316 \mathrm{SS}$ & $\mathrm{g} / 1 \mathrm{~g}$ of $316 \mathrm{SS}$ & $\mathrm{g} / 1 \mathrm{~g}$ of $316 \mathrm{SS}$ & $\mathrm{g} / 1 \mathrm{~g}$ of $316 \mathrm{SS}$ & $\mathrm{g} / 1 \mathrm{~g}$ of $316 \mathrm{SS}$ \\
\hline N 15 & $3.99 \mathrm{E}-06$ & $3.99 \mathrm{E}-06$ & $3.99 \mathrm{E}-06$ & $3.99 \mathrm{E}-06$ & $3.99 \mathrm{E}-06$ & $3.99 \mathrm{E}-06$ & 3.99E-06 & $3.99 \mathrm{E}-06$ & $3.99 \mathrm{E}-06$ & $3.99 \mathrm{E}-06$ \\
\hline 016 & $9.33 \mathrm{E}-14$ & $9.33 \mathrm{E}-14$ & $9.33 \mathrm{E}-14$ & $9.33 \mathrm{E}-14$ & $9.33 \mathrm{E}-14$ & $9.33 \mathrm{E}-14$ & $9.33 \mathrm{E}-14$ & $9.33 \mathrm{E}-14$ & $9.33 \mathrm{E}-14$ & $9.33 \mathrm{E}-14$ \\
\hline NE 22 & $3.21 \mathrm{E}-13$ & $3.21 \mathrm{E}-13$ & $3.21 \mathrm{E}-13$ & $3.21 \mathrm{E}-13$ & $3.21 \mathrm{E}-13$ & $3.21 \mathrm{E}-13$ & $3.21 \mathrm{E}-13$ & $3.21 \mathrm{E}-13$ & $3.21 \mathrm{E}-13$ & $3.21 \mathrm{E}-13$ \\
\hline NA 23 & $2.60 \mathrm{E}-14$ & $2.60 \mathrm{E}-14$ & $2.60 \mathrm{E}-14$ & $2.60 \mathrm{E}-14$ & $2.60 \mathrm{E}-14$ & $2.60 \mathrm{E}-14$ & $2.60 \mathrm{E}-14$ & $2.60 \mathrm{E}-14$ & $2.60 \mathrm{E}-14$ & $2.60 \mathrm{E}-14$ \\
\hline MG 25 & $1.40 \mathrm{E}-08$ & $1.40 \mathrm{E}-08$ & $1.40 \mathrm{E}-08$ & $1.40 \mathrm{E}-08$ & $1.40 \mathrm{E}-08$ & $1.40 \mathrm{E}-08$ & $1.40 \mathrm{E}-08$ & $1.40 \mathrm{E}-08$ & $1.40 \mathrm{E}-08$ & $1.40 \mathrm{E}-08$ \\
\hline MG 26 & $6.37 \mathrm{E}-09$ & $6.37 \mathrm{E}-09$ & $6.37 \mathrm{E}-09$ & $6.37 \mathrm{E}-09$ & $6.37 \mathrm{E}-09$ & $6.37 \mathrm{E}-09$ & $6.37 \mathrm{E}-09$ & $6.37 \mathrm{E}-09$ & $6.37 \mathrm{E}-09$ & $6.37 \mathrm{E}-09$ \\
\hline MG 27 & $2.97 \mathrm{E}-15$ & $3.30 \mathrm{E}-16$ & $0.00 \mathrm{E}+00$ & $0.00 \mathrm{E}+00$ & $0.00 \mathrm{E}+00$ & $0.00 \mathrm{E}+00$ & $0.00 \mathrm{E}+00$ & $0.00 \mathrm{E}+00$ & $0.00 \mathrm{E}+00$ & $0.00 \mathrm{E}+00$ \\
\hline AL 27 & $6.87 \mathrm{E}-11$ & $6.87 \mathrm{E}-11$ & $6.87 \mathrm{E}-11$ & $6.87 \mathrm{E}-11$ & $6.87 \mathrm{E}-11$ & $6.87 \mathrm{E}-11$ & $6.87 \mathrm{E}-11$ & $6.87 \mathrm{E}-11$ & $6.87 \mathrm{E}-11$ & $6.87 \mathrm{E}-11$ \\
\hline AL 28 & $8.82 \mathrm{E}-13$ & $8.20 \mathrm{E}-17$ & $5.26 \mathrm{E}-25$ & $4.45 \mathrm{E}-27$ & $0.00 \mathrm{E}+00$ & $0.00 \mathrm{E}+00$ & $0.00 \mathrm{E}+00$ & $0.00 \mathrm{E}+00$ & $0.00 \mathrm{E}+00$ & $0.00 \mathrm{E}+00$ \\
\hline AL 29 & $8.95 \mathrm{E}-14$ & $3.69 \mathrm{E}-15$ & $0.00 \mathrm{E}+00$ & $0.00 \mathrm{E}+00$ & $0.00 \mathrm{E}+00$ & $0.00 \mathrm{E}+00$ & $0.00 \mathrm{E}+00$ & $0.00 \mathrm{E}+00$ & $0.00 \mathrm{E}+00$ & $0.00 \mathrm{E}+00$ \\
\hline SI 28 & $6.89 \mathrm{E}-03$ & $6.89 \mathrm{E}-03$ & $6.89 \mathrm{E}-03$ & $6.89 \mathrm{E}-03$ & $6.89 \mathrm{E}-03$ & $6.89 \mathrm{E}-03$ & $6.89 \mathrm{E}-03$ & $6.89 \mathrm{E}-03$ & $6.89 \mathrm{E}-03$ & $6.89 \mathrm{E}-03$ \\
\hline SI 29 & $3.62 \mathrm{E}-04$ & $3.62 \mathrm{E}-04$ & $3.62 \mathrm{E}-04$ & $3.62 \mathrm{E}-04$ & $3.62 \mathrm{E}-04$ & $3.62 \mathrm{E}-04$ & $3.62 \mathrm{E}-04$ & $3.62 \mathrm{E}-04$ & $3.62 \mathrm{E}-04$ & $3.62 \mathrm{E}-04$ \\
\hline SI 30 & $2.48 \mathrm{E}-04$ & $2.48 \mathrm{E}-04$ & $2.48 \mathrm{E}-04$ & $2.48 \mathrm{E}-04$ & $2.48 \mathrm{E}-04$ & $2.48 \mathrm{E}-04$ & $2.48 \mathrm{E}-04$ & $2.48 \mathrm{E}-04$ & $2.48 \mathrm{E}-04$ & $2.48 \mathrm{E}-04$ \\
\hline SI 31 & $5.16 \mathrm{E}-11$ & $4.52 \mathrm{E}-11$ & $9.05 \mathrm{E}-14$ & $2.64 \mathrm{E}-30$ & $0.00 \mathrm{E}+00$ & $0.00 \mathrm{E}+00$ & $0.00 \mathrm{E}+00$ & $0.00 \mathrm{E}+00$ & $0.00 \mathrm{E}+00$ & $0.00 \mathrm{E}+00$ \\
\hline SI 32 & $2.35 \mathrm{E}-14$ & $2.35 \mathrm{E}-14$ & $2.35 \mathrm{E}-14$ & $2.35 \mathrm{E}-14$ & $2.35 \mathrm{E}-14$ & $2.35 \mathrm{E}-14$ & $2.35 \mathrm{E}-14$ & $2.35 \mathrm{E}-14$ & $2.35 \mathrm{E}-14$ & $2.35 \mathrm{E}-14$ \\
\hline P 31 & $4.50 \mathrm{E}-04$ & $4.50 \mathrm{E}-04$ & $4.50 \mathrm{E}-04$ & 4.50E-04 & $4.50 \mathrm{E}-04$ & $4.50 \mathrm{E}-04$ & $4.50 \mathrm{E}-04$ & $4.50 \mathrm{E}-04$ & 4.50E-04 & $4.50 \mathrm{E}-04$ \\
\hline P 32 & $1.22 \mathrm{E}-08$ & $1.21 \mathrm{E}-08$ & $1.16 \mathrm{E}-08$ & $8.66 \mathrm{E}-09$ & $2.84 \mathrm{E}-09$ & $6.63 \mathrm{E}-10$ & $1.55 \mathrm{E}-10$ & $3.62 \mathrm{E}-11$ & $7.49 \mathrm{E}-13$ & $3.22 \mathrm{E}-16$ \\
\hline P 33 & $7.35 \mathrm{E}-13$ & $7.35 \mathrm{E}-13$ & $7.15 \mathrm{E}-13$ & $6.06 \mathrm{E}-13$ & $3.20 \mathrm{E}-13$ & $1.39 \mathrm{E}-13$ & $6.06 \mathrm{E}-14$ & $2.64 \mathrm{E}-14$ & $2.87 \mathrm{E}-15$ & $3.40 \mathrm{E}-17$ \\
\hline S 32 & $2.84 \mathrm{E}-04$ & $2.84 \mathrm{E}-04$ & $2.84 \mathrm{E}-04$ & $2.84 \mathrm{E}-04$ & $2.84 \mathrm{E}-04$ & $2.84 \mathrm{E}-04$ & $2.84 \mathrm{E}-04$ & $2.84 \mathrm{E}-04$ & $2.84 \mathrm{E}-04$ & $2.84 \mathrm{E}-04$ \\
\hline S 33 & $2.46 \mathrm{E}-06$ & $2.46 \mathrm{E}-06$ & $2.46 \mathrm{E}-06$ & $2.46 \mathrm{E}-06$ & $2.46 \mathrm{E}-06$ & $2.46 \mathrm{E}-06$ & $2.46 \mathrm{E}-06$ & $2.46 \mathrm{E}-06$ & $2.46 \mathrm{E}-06$ & $2.46 \mathrm{E}-06$ \\
\hline S 34 & $1.34 \mathrm{E}-05$ & $1.34 \mathrm{E}-05$ & $1.34 \mathrm{E}-05$ & $1.34 \mathrm{E}-05$ & $1.34 \mathrm{E}-05$ & $1.34 \mathrm{E}-05$ & $1.34 \mathrm{E}-05$ & $1.34 \mathrm{E}-05$ & $1.34 \mathrm{E}-05$ & $1.34 \mathrm{E}-05$ \\
\hline S 35 & $1.45 \mathrm{E}-09$ & $1.45 \mathrm{E}-09$ & 1.44E-09 & 1.37E-09 & $1.15 \mathrm{E}-09$ & $9.04 \mathrm{E}-10$ & $7.14 \mathrm{E}-10$ & 5.64E-10 & $3.00 \mathrm{E}-10$ & $8.51 \mathrm{E}-11$ \\
\hline S 36 & $5.72 \mathrm{E}-08$ & $5.72 \mathrm{E}-08$ & $5.72 \mathrm{E}-08$ & $5.72 \mathrm{E}-08$ & $5.72 \mathrm{E}-08$ & $5.72 \mathrm{E}-08$ & $5.72 \mathrm{E}-08$ & $5.72 \mathrm{E}-08$ & $5.72 \mathrm{E}-08$ & $5.72 \mathrm{E}-08$ \\
\hline CL 35 & $1.57 \mathrm{E}-09$ & $1.57 \mathrm{E}-09$ & $1.59 \mathrm{E}-09$ & $1.65 \mathrm{E}-09$ & $1.88 \mathrm{E}-09$ & $2.12 \mathrm{E}-09$ & $2.31 \mathrm{E}-09$ & $2.46 \mathrm{E}-09$ & $2.72 \mathrm{E}-09$ & $2.94 \mathrm{E}-09$ \\
\hline $\mathrm{CL} 36$ & $2.38 \mathrm{E}-11$ & $2.38 \mathrm{E}-11$ & $2.38 \mathrm{E}-11$ & $2.38 \mathrm{E}-11$ & $2.38 \mathrm{E}-11$ & $2.38 \mathrm{E}-11$ & $2.38 \mathrm{E}-11$ & $2.38 \mathrm{E}-11$ & $2.38 \mathrm{E}-11$ & $2.38 \mathrm{E}-11$ \\
\hline CL 37 & $8.19 \mathrm{E}-12$ & $8.19 \mathrm{E}-12$ & $8.19 \mathrm{E}-12$ & $8.19 \mathrm{E}-12$ & $8.19 \mathrm{E}-12$ & $8.19 \mathrm{E}-12$ & $8.19 \mathrm{E}-12$ & $8.19 \mathrm{E}-12$ & $8.19 \mathrm{E}-12$ & $8.19 \mathrm{E}-12$ \\
\hline AR 38 & $1.77 \mathrm{E}-15$ & $1.77 \mathrm{E}-15$ & $1.77 \mathrm{E}-15$ & $1.77 \mathrm{E}-15$ & $1.77 \mathrm{E}-15$ & $1.77 \mathrm{E}-15$ & $1.77 \mathrm{E}-15$ & $1.77 \mathrm{E}-15$ & $1.77 \mathrm{E}-15$ & $1.77 \mathrm{E}-15$ \\
\hline CA 44 & $1.11 \mathrm{E}-13$ & $1.11 \mathrm{E}-13$ & $1.11 \mathrm{E}-13$ & $1.11 \mathrm{E}-13$ & $1.11 \mathrm{E}-13$ & $1.11 \mathrm{E}-13$ & $1.11 \mathrm{E}-13$ & $1.11 \mathrm{E}-13$ & $1.11 \mathrm{E}-13$ & $1.11 \mathrm{E}-13$ \\
\hline CA 46 & 4.44E-14 & 4.44E-14 & 4.44E-14 & 4.44E-14 & 4.44E-14 & 4.44E-14 & 4.44E-14 & $4.44 \mathrm{E}-14$ & 4.44E-14 & 4.44E-14 \\
\hline SC 47 & $2.04 \mathrm{E}-14$ & $2.03 \mathrm{E}-14$ & $1.66 \mathrm{E}-14$ & $4.79 \mathrm{E}-15$ & $4.12 \mathrm{E}-17$ & $8.46 \mathrm{E}-20$ & $1.85 \mathrm{E}-22$ & $0.00 \mathrm{E}+00$ & $4.49 \mathrm{E}-29$ & $0.00 \mathrm{E}+00$ \\
\hline SC 48 & $1.12 \mathrm{E}-14$ & $1.11 \mathrm{E}-14$ & $7.63 \mathrm{E}-15$ & $7.81 \mathrm{E}-16$ & $1.26 \mathrm{E}-19$ & $1.42 \mathrm{E}-24$ & $1.60 \mathrm{E}-29$ & $1.80 \mathrm{E}-34$ & $0.00 \mathrm{E}+00$ & $0.00 \mathrm{E}+00$ \\
\hline TI 47 & $1.40 \mathrm{E}-08$ & $1.40 \mathrm{E}-08$ & $1.40 \mathrm{E}-08$ & 1.40E-08 & $1.40 \mathrm{E}-08$ & $1.40 \mathrm{E}-08$ & 1.40E-08 & $1.40 \mathrm{E}-08$ & $1.40 \mathrm{E}-08$ & $1.40 \mathrm{E}-08$ \\
\hline TI 48 & $1.34 \mathrm{E}-11$ & $1.34 \mathrm{E}-11$ & 1.34E-11 & $1.34 \mathrm{E}-11$ & $1.34 \mathrm{E}-11$ & 1.34E-11 & 1.34E-11 & $1.34 \mathrm{E}-11$ & $1.34 \mathrm{E}-11$ & 1.34E-11 \\
\hline TI 49 & $3.81 \mathrm{E}-08$ & $3.81 \mathrm{E}-08$ & $3.81 \mathrm{E}-08$ & $3.81 \mathrm{E}-08$ & $3.81 \mathrm{E}-08$ & $3.81 \mathrm{E}-08$ & $3.81 \mathrm{E}-08$ & $3.81 \mathrm{E}-08$ & $3.81 \mathrm{E}-08$ & $3.81 \mathrm{E}-08$ \\
\hline TI 50 & $1.52 \mathrm{E}-07$ & $1.52 \mathrm{E}-07$ & $1.52 \mathrm{E}-07$ & $1.52 \mathrm{E}-07$ & $1.52 \mathrm{E}-07$ & $1.52 \mathrm{E}-07$ & $1.52 \mathrm{E}-07$ & $1.52 \mathrm{E}-07$ & $1.52 \mathrm{E}-07$ & $1.52 \mathrm{E}-07$ \\
\hline TI 51 & $1.80 \mathrm{E}-14$ & $4.86 \mathrm{E}-16$ & $0.00 \mathrm{E}+00$ & $0.00 \mathrm{E}+00$ & $0.00 \mathrm{E}+00$ & $0.00 \mathrm{E}+00$ & $0.00 \mathrm{E}+00$ & $0.00 \mathrm{E}+00$ & $0.00 E+00$ & $0.00 \mathrm{E}+00$ \\
\hline V 50 & $5.60 \mathrm{E}-07$ & $5.60 \mathrm{E}-07$ & $5.60 \mathrm{E}-07$ & $5.60 \mathrm{E}-07$ & 5.60E-07 & $5.60 \mathrm{E}-07$ & $5.60 \mathrm{E}-07$ & $5.60 \mathrm{E}-07$ & $5.60 \mathrm{E}-07$ & $5.60 \mathrm{E}-07$ \\
\hline
\end{tabular}


TEM-10200-1

$12 / 19 / 17$

Rev.08

Title:

AS-RUN NEUTRONICS EVALUATION FOR THE GE HITACHI EXPERIMENT IN THE ATR

ECAR NO.: $4740 \quad$ REV. NO.: 0

\begin{tabular}{|c|c|c|c|c|c|c|c|c|c|c|}
\hline \multicolumn{11}{|c|}{$1 \mathrm{~g}$ of $316 \mathrm{SS}$} \\
\hline Iccton & EOI & $30.0 \mathrm{Ml}$ & $1.0 \mathrm{D}$ & $7.0 \mathrm{D}$ & $30.0 \mathrm{D}$ & $60.0 \mathrm{D}$ & $90.0 \mathrm{D}$ & $120.0 \mathrm{D}$ & $200.0 \mathrm{D}$ & $360.0 \mathrm{D}$ \\
\hline Isotope & $\mathrm{g} / 1 \mathrm{~g}$ of $316 \mathrm{SS}$ & $\mathrm{g} / 1 \mathrm{~g}$ of $316 \mathrm{SS}$ & $\mathrm{g} / 1 \mathrm{~g}$ of $316 \mathrm{SS}$ & $\mathrm{g} / 1 \mathrm{~g}$ of $316 \mathrm{SS}$ & $\mathrm{g} / 1 \mathrm{~g}$ of $316 \mathrm{SS}$ & $\mathrm{g} / 1 \mathrm{~g}$ of $316 \mathrm{SS}$ & $\mathrm{g} / 1 \mathrm{~g}$ of $316 \mathrm{SS}$ & $\mathrm{g} / 1 \mathrm{~g}$ of $316 \mathrm{SS}$ & $\mathrm{g} / 1 \mathrm{~g}$ of $316 \mathrm{SS}$ & $\mathrm{g} / 1 \mathrm{~g}$ of $316 \mathrm{SS}$ \\
\hline V 51 & $9.21 \mathrm{E}-05$ & $9.21 \mathrm{E}-05$ & $9.26 \mathrm{E}-05$ & 9.54E-05 & 1.03E-04 & $1.08 \mathrm{E}-04$ & 1.10E-04 & $1.12 \mathrm{E}-04$ & $1.12 \mathrm{E}-04$ & 1.13E-04 \\
\hline$V 52$ & 1.97E-11 & $7.68 \mathrm{E}-14$ & $0.00 \mathrm{E}+00$ & $0.00 \mathrm{E}+00$ & $0.00 \mathrm{E}+00$ & $0.00 \mathrm{E}+00$ & $0.00 \mathrm{E}+00$ & $0.00 \mathrm{E}+00$ & $0.00 \mathrm{E}+00$ & $0.00 \mathrm{E}+00$ \\
\hline V 53 & $3.95 \mathrm{E}-14$ & $9.70 \mathrm{E}-20$ & $0.00 \mathrm{E}+00$ & $0.00 \mathrm{E}+00$ & $0.00 \mathrm{E}+00$ & $0.00 \mathrm{E}+00$ & $0.00 \mathrm{E}+00$ & $0.00 \mathrm{E}+00$ & $0.00 \mathrm{E}+00$ & $0.00 \mathrm{E}+00$ \\
\hline CR 50 & $7.41 \mathrm{E}-03$ & $7.41 \mathrm{E}-03$ & $7.41 \mathrm{E}-03$ & $7.41 \mathrm{E}-03$ & $7.41 \mathrm{E}-03$ & $7.41 \mathrm{E}-03$ & $7.41 \mathrm{E}-03$ & $7.41 \mathrm{E}-03$ & $7.41 \mathrm{E}-03$ & $7.41 \mathrm{E}-03$ \\
\hline CR 51 & $2.03 \mathrm{E}-05$ & $2.03 \mathrm{E}-05$ & $1.98 \mathrm{E}-05$ & $1.71 \mathrm{E}-05$ & $9.60 \mathrm{E}-06$ & $4.53 \mathrm{E}-06$ & $2.14 \mathrm{E}-06$ & $1.01 \mathrm{E}-06$ & $1.37 \mathrm{E}-07$ & $2.50 \mathrm{E}-09$ \\
\hline CR 52 & $1.50 \mathrm{E}-01$ & $1.50 \mathrm{E}-01$ & $1.50 \mathrm{E}-01$ & $1.50 \mathrm{E}-01$ & $1.50 \mathrm{E}-01$ & $1.50 \mathrm{E}-01$ & $1.50 \mathrm{E}-01$ & $1.50 \mathrm{E}-01$ & 1.50E-01 & $1.50 \mathrm{E}-01$ \\
\hline CR 53 & $1.76 \mathrm{E}-02$ & $1.76 \mathrm{E}-02$ & $1.76 \mathrm{E}-02$ & $1.76 \mathrm{E}-02$ & $1.76 \mathrm{E}-02$ & $1.76 \mathrm{E}-02$ & $1.76 \mathrm{E}-02$ & $1.76 \mathrm{E}-02$ & $1.76 \mathrm{E}-02$ & $1.76 \mathrm{E}-02$ \\
\hline CR 54 & $4.71 \mathrm{E}-03$ & $4.71 \mathrm{E}-03$ & $4.71 \mathrm{E}-03$ & $4.71 \mathrm{E}-03$ & $4.71 \mathrm{E}-03$ & $4.71 \mathrm{E}-03$ & $4.71 \mathrm{E}-03$ & $4.71 \mathrm{E}-03$ & $4.71 \mathrm{E}-03$ & $4.71 \mathrm{E}-03$ \\
\hline CR 55 & $2.73 \mathrm{E}-11$ & $7.80 \mathrm{E}-14$ & $0.00 \mathrm{E}+00$ & $0.00 \mathrm{E}+00$ & $0.00 \mathrm{E}+00$ & $0.00 \mathrm{E}+00$ & $0.00 \mathrm{E}+00$ & $0.00 \mathrm{E}+00$ & $0.00 \mathrm{E}+00$ & $0.00 \mathrm{E}+00$ \\
\hline MN 54 & $5.56 \mathrm{E}-06$ & $5.56 \mathrm{E}-06$ & $5.55 \mathrm{E}-06$ & 5.47E-06 & $5.20 \mathrm{E}-06$ & 4.87E-06 & $4.55 \mathrm{E}-06$ & $4.26 \mathrm{E}-06$ & 3.57E-06 & $2.50 \mathrm{E}-06$ \\
\hline MN 55 & $1.97 \mathrm{E}-02$ & $1.97 \mathrm{E}-02$ & $1.97 \mathrm{E}-02$ & 1.97E-02 & 1.97E-02 & $1.97 \mathrm{E}-02$ & 1.97E-02 & 1.97E-02 & $1.97 \mathrm{E}-02$ & $1.97 \mathrm{E}-02$ \\
\hline MN 56 & $2.23 \mathrm{E}-07$ & $1.95 \mathrm{E}-07$ & $3.53 \mathrm{E}-10$ & $5.45 \mathrm{E}-27$ & $0.00 \mathrm{E}+00$ & $0.00 \mathrm{E}+00$ & $0.00 \mathrm{E}+00$ & $0.00 \mathrm{E}+00$ & $0.00 \mathrm{E}+00$ & $0.00 \mathrm{E}+00$ \\
\hline MN 57 & $1.37 \mathrm{E}-13$ & $3.37 \mathrm{E}-19$ & $0.00 \mathrm{E}+00$ & $0.00 \mathrm{E}+00$ & $0.00 \mathrm{E}+00$ & $0.00 \mathrm{E}+00$ & $0.00 \mathrm{E}+00$ & $0.00 \mathrm{E}+00$ & $0.00 \mathrm{E}+00$ & $0.00 \mathrm{E}+00$ \\
\hline FE 54 & $3.47 \mathrm{E}-02$ & $3.47 \mathrm{E}-02$ & $3.47 \mathrm{E}-02$ & $3.47 \mathrm{E}-02$ & 3.47E-02 & $3.47 \mathrm{E}-02$ & $3.47 \mathrm{E}-02$ & 3.47E-02 & $3.47 \mathrm{E}-02$ & $3.47 \mathrm{E}-02$ \\
\hline FE 55 & 7.04E-05 & $7.04 \mathrm{E}-05$ & $7.04 \mathrm{E}-05$ & $7.01 \mathrm{E}-05$ & $6.89 \mathrm{E}-05$ & $6.74 \mathrm{E}-05$ & $6.59 \mathrm{E}-05$ & $6.45 \mathrm{E}-05$ & $6.09 \mathrm{E}-05$ & 5.41E-05 \\
\hline FE 56 & $5.69 \mathrm{E}-01$ & $5.69 \mathrm{E}-01$ & $5.69 \mathrm{E}-01$ & $5.69 \mathrm{E}-01$ & 5.69E-01 & 5.69E-01 & 5.69E-01 & 5.69E-01 & 5.69E-01 & 5.69E-01 \\
\hline FE 57 & $1.51 \mathrm{E}-02$ & $1.51 \mathrm{E}-02$ & $1.51 \mathrm{E}-02$ & $1.51 \mathrm{E}-02$ & $1.51 \mathrm{E}-02$ & $1.51 \mathrm{E}-02$ & $1.51 \mathrm{E}-02$ & $1.51 \mathrm{E}-02$ & $1.51 \mathrm{E}-02$ & $1.51 \mathrm{E}-02$ \\
\hline FE 58 & $1.90 \mathrm{E}-03$ & $1.90 \mathrm{E}-03$ & $1.90 \mathrm{E}-03$ & $1.90 \mathrm{E}-03$ & $1.90 \mathrm{E}-03$ & $1.91 \mathrm{E}-03$ & 1.91E-03 & $1.91 \mathrm{E}-03$ & $1.91 \mathrm{E}-03$ & $1.91 \mathrm{E}-03$ \\
\hline FE 59 & $6.75 \mathrm{E}-07$ & $6.74 \mathrm{E}-07$ & $6.64 \mathrm{E}-07$ & $6.06 \mathrm{E}-07$ & $4.25 \mathrm{E}-07$ & $2.68 \mathrm{E}-07$ & 1.69E-07 & $1.06 \mathrm{E}-07$ & $3.10 \mathrm{E}-08$ & $2.64 \mathrm{E}-09$ \\
\hline $\mathrm{CO} 58$ & $3.66 \mathrm{E}-06$ & $3.66 \mathrm{E}-06$ & $3.62 \mathrm{E}-06$ & $3.42 \mathrm{E}-06$ & $2.73 \mathrm{E}-06$ & $2.03 E-06$ & 1.52E-06 & $1.13 E-06$ & $5.16 \mathrm{E}-07$ & $1.08 \mathrm{E}-07$ \\
\hline CO 59 & $1.13 \mathrm{E}-05$ & $1.13 \mathrm{E}-05$ & $1.13 \mathrm{E}-05$ & $1.13 \mathrm{E}-05$ & $1.15 \mathrm{E}-05$ & $1.17 \mathrm{E}-05$ & $1.18 \mathrm{E}-05$ & $1.18 \mathrm{E}-05$ & $1.19 \mathrm{E}-05$ & $1.19 \mathrm{E}-05$ \\
\hline $\mathrm{CO} 60$ & $4.52 \mathrm{E}-07$ & $4.52 \mathrm{E}-07$ & $4.51 \mathrm{E}-07$ & 4.50E-07 & 4.47E-07 & $4.42 \mathrm{E}-07$ & 4.37E-07 & 4.32E-07 & $4.20 \mathrm{E}-07$ & $3.97 \mathrm{E}-07$ \\
\hline CO 60M & $1.44 \mathrm{E}-11$ & $1.97 \mathrm{E}-12$ & $0.00 \mathrm{E}+00$ & $0.00 \mathrm{E}+00$ & $0.00 \mathrm{E}+00$ & $0.00 \mathrm{E}+00$ & $0.00 \mathrm{E}+00$ & $0.00 \mathrm{E}+00$ & $0.00 \mathrm{E}+00$ & $0.00 \mathrm{E}+00$ \\
\hline $\mathrm{CO} 61$ & $4.66 \mathrm{E}-12$ & $3.78 \mathrm{E}-12$ & $1.95 \mathrm{E}-16$ & $0.00 \mathrm{E}+00$ & $0.00 \mathrm{E}+00$ & $0.00 \mathrm{E}+00$ & $0.00 \mathrm{E}+00$ & $0.00 \mathrm{E}+00$ & $0.00 E+00$ & $0.00 \mathrm{E}+00$ \\
\hline CO 62 & $1.10 \mathrm{E}-14$ & $1.05 \mathrm{E}-20$ & $0.00 \mathrm{E}+00$ & $0.00 \mathrm{E}+00$ & $0.00 \mathrm{E}+00$ & $0.00 \mathrm{E}+00$ & $0.00 \mathrm{E}+00$ & $0.00 \mathrm{E}+00$ & $0.00 \mathrm{E}+00$ & $0.00 \mathrm{E}+00$ \\
\hline NI 58 & $9.39 \mathrm{E}-02$ & $9.39 \mathrm{E}-02$ & $9.39 \mathrm{E}-02$ & $9.39 \mathrm{E}-02$ & $9.39 \mathrm{E}-02$ & $9.39 \mathrm{E}-02$ & $9.39 \mathrm{E}-02$ & $9.39 \mathrm{E}-02$ & $9.39 \mathrm{E}-02$ & $9.39 \mathrm{E}-02$ \\
\hline NI 59 & $4.40 \mathrm{E}-04$ & $4.40 \mathrm{E}-04$ & $4.40 \mathrm{E}-04$ & $4.40 \mathrm{E}-04$ & $4.40 \mathrm{E}-04$ & $4.40 \mathrm{E}-04$ & $4.40 \mathrm{E}-04$ & 4.40E-04 & 4.40E-04 & $4.40 \mathrm{E}-04$ \\
\hline NI 60 & $3.72 \mathrm{E}-02$ & $3.72 \mathrm{E}-02$ & $3.72 \mathrm{E}-02$ & $3.72 \mathrm{E}-02$ & $3.72 \mathrm{E}-02$ & $3.72 \mathrm{E}-02$ & $3.72 \mathrm{E}-02$ & $3.72 \mathrm{E}-02$ & $3.72 \mathrm{E}-02$ & $3.72 \mathrm{E}-02$ \\
\hline NI 61 & $1.74 \mathrm{E}-03$ & $1.74 \mathrm{E}-03$ & $1.74 \mathrm{E}-03$ & $1.74 \mathrm{E}-03$ & $1.74 \mathrm{E}-03$ & $1.74 \mathrm{E}-03$ & $1.74 \mathrm{E}-03$ & $1.74 \mathrm{E}-03$ & $1.74 \mathrm{E}-03$ & $1.74 \mathrm{E}-03$ \\
\hline NI 62 & $5.24 \mathrm{E}-03$ & $5.24 \mathrm{E}-03$ & $5.24 \mathrm{E}-03$ & $5.24 \mathrm{E}-03$ & $5.24 \mathrm{E}-03$ & $5.24 \mathrm{E}-03$ & $5.24 \mathrm{E}-03$ & $5.24 \mathrm{E}-03$ & $5.24 \mathrm{E}-03$ & $5.24 \mathrm{E}-03$ \\
\hline NI 63 & $6.97 \mathrm{E}-05$ & $6.97 \mathrm{E}-05$ & $6.97 \mathrm{E}-05$ & $6.97 \mathrm{E}-05$ & $6.97 \mathrm{E}-05$ & $6.96 \mathrm{E}-05$ & $6.96 \mathrm{E}-05$ & $6.95 \mathrm{E}-05$ & $6.94 \mathrm{E}-05$ & $6.92 \mathrm{E}-05$ \\
\hline NI 64 & 1.39E-03 & $1.39 \mathrm{E}-03$ & $1.39 \mathrm{E}-03$ & 1.39E-03 & $1.39 \mathrm{E}-03$ & $1.39 \mathrm{E}-03$ & $1.39 \mathrm{E}-03$ & $1.39 \mathrm{E}-03$ & $1.39 \mathrm{E}-03$ & $1.39 \mathrm{E}-03$ \\
\hline NI 65 & 1.44E-09 & $1.25 \mathrm{E}-09$ & 1.96E-12 & $1.23 \mathrm{E}-29$ & $0.00 \mathrm{E}+00$ & $0.00 \mathrm{E}+00$ & $0.00 \mathrm{E}+00$ & $0.00 \mathrm{E}+00$ & $0.00 \mathrm{E}+00$ & $0.00 \mathrm{E}+00$ \\
\hline NI 66 & $4.83 \mathrm{E}-13$ & $4.80 \mathrm{E}-13$ & $3.56 \mathrm{E}-13$ & $5.72 \mathrm{E}-14$ & $5.19 \mathrm{E}-17$ & $5.57 \mathrm{E}-21$ & $5.99 \mathrm{E}-25$ & $6.43 \mathrm{E}-29$ & $0.00 \mathrm{E}+00$ & $0.00 \mathrm{E}+00$ \\
\hline CU 63 & $1.59 \mathrm{E}-07$ & $1.59 \mathrm{E}-07$ & 1.60E-07 & 1.69E-07 & $2.02 \mathrm{E}-07$ & $2.45 \mathrm{E}-07$ & $2.88 \mathrm{E}-07$ & $3.31 \mathrm{E}-07$ & $4.45 \mathrm{E}-07$ & $6.74 \mathrm{E}-07$ \\
\hline CU 64 & $2.74 \mathrm{E}-12$ & $2.66 \mathrm{E}-12$ & $7.38 \mathrm{E}-13$ & $2.85 \mathrm{E}-16$ & $2.35 \mathrm{E}-29$ & $0.00 \mathrm{E}+00$ & $0.00 \mathrm{E}+00$ & $0.00 \mathrm{E}+00$ & $0.00 \mathrm{E}+00$ & $0.00 \mathrm{E}+00$ \\
\hline CU 65 & $2.08 \mathrm{E}-06$ & $2.08 \mathrm{E}-06$ & $2.08 \mathrm{E}-06$ & $2.08 \mathrm{E}-06$ & $2.08 \mathrm{E}-06$ & $2.08 \mathrm{E}-06$ & $2.08 \mathrm{E}-06$ & $2.08 \mathrm{E}-06$ & $2.08 \mathrm{E}-06$ & $2.08 \mathrm{E}-06$ \\
\hline
\end{tabular}

ENGINEERING CALCULATIONS AND ANALYSIS

Page $\mathbf{5 0}$ of $\mathbf{5 7}$ 
TEM-10200-1

$12 / 19 / 17$

Rev.08

Title:

AS-RUN NEUTRONICS EVALUATION FOR THE GE HITACHI EXPERIMENT IN THE ATR

ECAR NO.: $4740 \quad$ REV. NO.: 0

\begin{tabular}{|c|c|c|c|c|c|c|c|c|c|c|}
\hline \multicolumn{11}{|c|}{$1 \mathrm{~g}$ of $316 \mathrm{SS}$} \\
\hline Istono & EOI & $30.0 \mathrm{MI}$ & $1.0 \mathrm{D}$ & $7.0 \mathrm{D}$ & $30.0 \mathrm{D}$ & $60.0 \mathrm{D}$ & $90.0 \mathrm{D}$ & $120.0 \mathrm{D}$ & $200.0 \mathrm{D}$ & $360.0 \mathrm{D}$ \\
\hline Isotope & $\mathrm{g} / 1 \mathrm{~g}$ of $316 \mathrm{SS}$ & $\mathrm{g} / 1 \mathrm{~g}$ of $316 \mathrm{SS}$ & $\mathrm{g} / 1 \mathrm{~g}$ of $316 \mathrm{SS}$ & $\mathrm{g} / 1 \mathrm{~g}$ of $316 \mathrm{SS}$ & $\mathrm{g} / 1 \mathrm{~g}$ of $316 \mathrm{SS}$ & $\mathrm{g} / 1 \mathrm{~g}$ of $316 \mathrm{SS}$ & $\mathrm{g} / 1 \mathrm{~g}$ of $316 \mathrm{SS}$ & $\mathrm{g} / 1 \mathrm{~g}$ of $316 \mathrm{SS}$ & $\mathrm{g} / 1 \mathrm{~g}$ of $316 \mathrm{SS}$ & $\mathrm{g} / 1 \mathrm{~g}$ of $316 \mathrm{SS}$ \\
\hline CU 66 & $1.16 \mathrm{E}-13$ & $2.71 \mathrm{E}-15$ & $5.55 \mathrm{E}-16$ & $8.92 \mathrm{E}-17$ & $8.08 \mathrm{E}-20$ & $8.68 \mathrm{E}-24$ & $9.33 \mathrm{E}-28$ & 1.00E-31 & $0.00 \mathrm{E}+00$ & $0.00 \mathrm{E}+00$ \\
\hline $\mathrm{ZN} 64$ & $9.76 \mathrm{E}-11$ & $9.76 \mathrm{E}-11$ & $9.83 \mathrm{E}-11$ & $9.86 \mathrm{E}-11$ & $9.86 \mathrm{E}-11$ & $9.86 \mathrm{E}-11$ & $9.86 \mathrm{E}-11$ & $9.86 \mathrm{E}-11$ & $9.86 \mathrm{E}-11$ & $9.86 \mathrm{E}-11$ \\
\hline ZN 65 & $2.17 \mathrm{E}-14$ & $2.17 \mathrm{E}-14$ & $2.16 \mathrm{E}-14$ & $2.12 \mathrm{E}-14$ & $1.99 \mathrm{E}-14$ & $1.83 \mathrm{E}-14$ & $1.68 \mathrm{E}-14$ & $1.54 \mathrm{E}-14$ & $1.23 \mathrm{E}-14$ & $7.79 \mathrm{E}-15$ \\
\hline ZN 66 & $2.51 \mathrm{E}-09$ & $2.51 \mathrm{E}-09$ & $2.51 \mathrm{E}-09$ & $2.51 \mathrm{E}-09$ & $2.51 \mathrm{E}-09$ & $2.51 \mathrm{E}-09$ & $2.51 \mathrm{E}-09$ & $2.51 \mathrm{E}-09$ & $2.51 \mathrm{E}-09$ & $2.51 \mathrm{E}-09$ \\
\hline ZN 67 & $7.63 \mathrm{E}-13$ & $7.63 \mathrm{E}-13$ & $7.63 \mathrm{E}-13$ & $7.63 \mathrm{E}-13$ & $7.64 \mathrm{E}-13$ & $7.64 \mathrm{E}-13$ & $7.64 \mathrm{E}-13$ & $7.64 \mathrm{E}-13$ & $7.64 \mathrm{E}-13$ & $7.64 \mathrm{E}-13$ \\
\hline ZN 68 & $2.06 \mathrm{E}-15$ & $2.06 \mathrm{E}-15$ & $2.06 \mathrm{E}-15$ & $2.06 \mathrm{E}-15$ & $2.06 \mathrm{E}-15$ & $2.06 \mathrm{E}-15$ & $2.06 \mathrm{E}-15$ & $2.06 \mathrm{E}-15$ & $2.06 \mathrm{E}-15$ & $2.06 \mathrm{E}-15$ \\
\hline SR 88 & $1.19 \mathrm{E}-14$ & $1.19 \mathrm{E}-14$ & 1.19E-14 & $1.19 \mathrm{E}-14$ & $1.19 \mathrm{E}-14$ & $1.19 \mathrm{E}-14$ & $1.19 \mathrm{E}-14$ & $1.19 \mathrm{E}-14$ & $1.19 \mathrm{E}-14$ & $1.19 \mathrm{E}-14$ \\
\hline SR 89 & $6.21 \mathrm{E}-15$ & $6.21 \mathrm{E}-15$ & $6.13 \mathrm{E}-15$ & $5.65 \mathrm{E}-15$ & $4.12 \mathrm{E}-15$ & $2.73 \mathrm{E}-15$ & $1.81 \mathrm{E}-15$ & $1.20 \mathrm{E}-15$ & $3.99 \mathrm{E}-16$ & 4.44E-17 \\
\hline Y 89 & $3.85 \mathrm{E}-10$ & $3.85 \mathrm{E}-10$ & $3.86 \mathrm{E}-10$ & $3.91 \mathrm{E}-10$ & $3.93 \mathrm{E}-10$ & $3.93 \mathrm{E}-10$ & $3.93 \mathrm{E}-10$ & $3.93 \mathrm{E}-10$ & $3.93 \mathrm{E}-10$ & $3.93 \mathrm{E}-10$ \\
\hline Y 90 & $8.98 \mathrm{E}-15$ & $8.93 E-15$ & $6.92 \mathrm{E}-15$ & $1.46 \mathrm{E}-15$ & $3.77 \mathrm{E}-18$ & $8.53 \mathrm{E}-20$ & $8.37 \mathrm{E}-20$ & $8.35 \mathrm{E}-20$ & $8.30 \mathrm{E}-20$ & $8.22 \mathrm{E}-20$ \\
\hline Y91 & $1.20 \mathrm{E}-15$ & $1.20 \mathrm{E}-15$ & $1.18 \mathrm{E}-15$ & $1.10 \mathrm{E}-15$ & $8.38 \mathrm{E}-16$ & $5.87 \mathrm{E}-16$ & $4.12 \mathrm{E}-16$ & $2.89 \mathrm{E}-16$ & $1.12 \mathrm{E}-16$ & $1.68 \mathrm{E}-17$ \\
\hline ZR 89 & $8.46 \mathrm{E}-12$ & $8.42 \mathrm{E}-12$ & $6.84 \mathrm{E}-12$ & $1.92 \mathrm{E}-12$ & 1.46E-14 & $2.52 \mathrm{E}-17$ & $4.35 \mathrm{E}-20$ & $7.90 \mathrm{E}-23$ & $3.39 \mathrm{E}-30$ & $0.00 \mathrm{E}+00$ \\
\hline ZR 90 & $2.50 \mathrm{E}-13$ & $2.50 \mathrm{E}-13$ & $2.52 \mathrm{E}-13$ & $2.57 \mathrm{E}-13$ & $2.59 \mathrm{E}-13$ & $2.59 \mathrm{E}-13$ & $2.59 \mathrm{E}-13$ & $2.59 \mathrm{E}-13$ & $2.59 \mathrm{E}-13$ & $2.59 \mathrm{E}-13$ \\
\hline ZR 91 & 5.35E-09 & $5.35 \mathrm{E}-09$ & $5.35 \mathrm{E}-09$ & 5.35E-09 & $5.35 \mathrm{E}-09$ & $5.35 \mathrm{E}-09$ & 5.35E-09 & 5.35E-09 & 5.35E-09 & $5.35 \mathrm{E}-09$ \\
\hline ZR 92 & $1.26 \mathrm{E}-07$ & $1.26 \mathrm{E}-07$ & $1.27 \mathrm{E}-07$ & $1.28 \mathrm{E}-07$ & $1.31 \mathrm{E}-07$ & $1.32 \mathrm{E}-07$ & $1.32 \mathrm{E}-07$ & $1.32 \mathrm{E}-07$ & $1.32 \mathrm{E}-07$ & $1.32 \mathrm{E}-07$ \\
\hline ZR 93 & $4.55 \mathrm{E}-09$ & $4.55 \mathrm{E}-09$ & $4.55 \mathrm{E}-09$ & $4.55 \mathrm{E}-09$ & $4.55 \mathrm{E}-09$ & $4.55 \mathrm{E}-09$ & 4.55E-09 & $4.55 \mathrm{E}-09$ & $4.55 \mathrm{E}-09$ & $4.55 \mathrm{E}-09$ \\
\hline ZR 94 & $1.13 \mathrm{E}-08$ & $1.13 \mathrm{E}-08$ & $1.13 \mathrm{E}-08$ & $1.13 \mathrm{E}-08$ & $1.13 \mathrm{E}-08$ & $1.13 \mathrm{E}-08$ & $1.13 \mathrm{E}-08$ & $1.13 \mathrm{E}-08$ & $1.13 \mathrm{E}-08$ & $1.13 \mathrm{E}-08$ \\
\hline ZR 95 & $5.54 \mathrm{E}-10$ & $5.53 \mathrm{E}-10$ & $5.48 \mathrm{E}-10$ & $5.13 \mathrm{E}-10$ & 4.00E-10 & $2.89 \mathrm{E}-10$ & $2.09 \mathrm{E}-10$ & $1.51 \mathrm{E}-10$ & $6.34 \mathrm{E}-11$ & 1.12E-11 \\
\hline ZR 96 & $8.87 \mathrm{E}-13$ & $8.87 \mathrm{E}-13$ & $8.87 \mathrm{E}-13$ & $8.87 \mathrm{E}-13$ & $8.87 E-13$ & $8.87 \mathrm{E}-13$ & $8.87 \mathrm{E}-13$ & $8.87 E-13$ & $8.87 \mathrm{E}-13$ & $8.87 \mathrm{E}-13$ \\
\hline ZR 97 & 1.57E-13 & $1.54 \mathrm{E}-13$ & 5.87E-14 & $1.60 \mathrm{E}-16$ & $2.35 \mathrm{E}-26$ & $0.00 \mathrm{E}+00$ & $0.00 \mathrm{E}+00$ & $0.00 \mathrm{E}+00$ & $0.00 \mathrm{E}+00$ & $0.00 \mathrm{E}+00$ \\
\hline NB 92 & $5.41 \mathrm{E}-09$ & $5.40 \mathrm{E}-09$ & $5.05 \mathrm{E}-09$ & $3.35 \mathrm{E}-09$ & $6.99 \mathrm{E}-10$ & $9.02 \mathrm{E}-11$ & 1.17E-11 & $1.51 \mathrm{E}-12$ & $6.42 \mathrm{E}-15$ & 1.17E-19 \\
\hline NB 93 & 4.35E-11 & $4.35 \mathrm{E}-11$ & $4.38 \mathrm{E}-11$ & $4.62 \mathrm{E}-11$ & $5.54 \mathrm{E}-11$ & $6.73 \mathrm{E}-11$ & $7.92 \mathrm{E}-11$ & $9.11 \mathrm{E}-11$ & $1.23 \mathrm{E}-10$ & 1.86E-10 \\
\hline NB 93M & $5.79 \mathrm{E}-16$ & $5.79 \mathrm{E}-16$ & $5.84 \mathrm{E}-16$ & $6.16 \mathrm{E}-16$ & $7.37 \mathrm{E}-16$ & $8.94 \mathrm{E}-16$ & $1.05 \mathrm{E}-15$ & $1.21 \mathrm{E}-15$ & $1.62 \mathrm{E}-15$ & $2.43 \mathrm{E}-15$ \\
\hline NB 94 & $6.81 \mathrm{E}-09$ & $6.81 \mathrm{E}-09$ & $6.81 \mathrm{E}-09$ & $6.81 \mathrm{E}-09$ & $6.81 \mathrm{E}-09$ & $6.81 \mathrm{E}-09$ & $6.81 \mathrm{E}-09$ & $6.81 \mathrm{E}-09$ & $6.81 \mathrm{E}-09$ & $6.81 \mathrm{E}-09$ \\
\hline NB 95 & $7.39 \mathrm{E}-10$ & $7.39 \mathrm{E}-10$ & $7.31 \mathrm{E}-10$ & $6.82 \mathrm{E}-10$ & $5.23 \mathrm{E}-10$ & $3.72 \mathrm{E}-10$ & $2.66 \mathrm{E}-10$ & $1.90 \mathrm{E}-10$ & $7.86 \mathrm{E}-11$ & $1.37 \mathrm{E}-11$ \\
\hline NB 95M & $2.17 \mathrm{E}-13$ & $2.17 \mathrm{E}-13$ & $2.17 \mathrm{E}-13$ & $2.11 \mathrm{E}-13$ & $1.67 \mathrm{E}-13$ & $1.21 \mathrm{E}-13$ & $8.74 \mathrm{E}-14$ & $6.31 \mathrm{E}-14$ & $2.65 \mathrm{E}-14$ & $4.69 \mathrm{E}-15$ \\
\hline NB 96 & $1.94 \mathrm{E}-11$ & $1.91 \mathrm{E}-11$ & $9.49 \mathrm{E}-12$ & $1.32 \mathrm{E}-13$ & $1.01 \mathrm{E}-20$ & $5.27 E-30$ & $0.00 \mathrm{E}+00$ & $0.00 \mathrm{E}+00$ & $0.00 \mathrm{E}+00$ & $0.00 \mathrm{E}+00$ \\
\hline NB 97 & $3.96 \mathrm{E}-13$ & $2.99 \mathrm{E}-13$ & $4.19 \mathrm{E}-15$ & 1.14E-17 & $1.80 \mathrm{E}-27$ & $0.00 \mathrm{E}+00$ & $0.00 \mathrm{E}+00$ & $0.00 \mathrm{E}+00$ & $0.00 \mathrm{E}+00$ & $0.00 \mathrm{E}+00$ \\
\hline MO 92 & $4.25 \mathrm{E}-03$ & $4.25 \mathrm{E}-03$ & $4.25 \mathrm{E}-03$ & $4.25 \mathrm{E}-03$ & 4.25E-03 & $4.25 \mathrm{E}-03$ & $4.25 \mathrm{E}-03$ & $4.25 \mathrm{E}-03$ & $4.25 \mathrm{E}-03$ & $4.25 \mathrm{E}-03$ \\
\hline MO 93M & 4.46E-11 & $4.24 \mathrm{E}-11$ & $3.93 E-12$ & $1.85 \mathrm{E}-18$ & $0.00 \mathrm{E}+00$ & $0.00 \mathrm{E}+00$ & $0.00 \mathrm{E}+00$ & $0.00 \mathrm{E}+00$ & $0.00 \mathrm{E}+00$ & $0.00 \mathrm{E}+00$ \\
\hline MO 93 & $7.31 \mathrm{E}-07$ & $7.31 \mathrm{E}-07$ & $7.31 \mathrm{E}-07$ & $7.31 \mathrm{E}-07$ & $7.31 \mathrm{E}-07$ & $7.31 \mathrm{E}-07$ & $7.31 \mathrm{E}-07$ & $7.31 \mathrm{E}-07$ & $7.31 \mathrm{E}-07$ & $7.31 \mathrm{E}-07$ \\
\hline MO 94 & $2.73 \mathrm{E}-03$ & $2.73 \mathrm{E}-03$ & $2.73 \mathrm{E}-03$ & $2.73 \mathrm{E}-03$ & $2.73 \mathrm{E}-03$ & $2.73 \mathrm{E}-03$ & $2.73 \mathrm{E}-03$ & $2.73 \mathrm{E}-03$ & $2.73 \mathrm{E}-03$ & $2.73 \mathrm{E}-03$ \\
\hline MO 95 & $4.53 \mathrm{E}-03$ & $4.53 \mathrm{E}-03$ & $4.53 \mathrm{E}-03$ & $4.53 \mathrm{E}-03$ & $4.53 \mathrm{E}-03$ & $4.53 \mathrm{E}-03$ & 4.53E-03 & $4.53 \mathrm{E}-03$ & $4.53 \mathrm{E}-03$ & $4.53 \mathrm{E}-03$ \\
\hline MO 96 & $5.17 \mathrm{E}-03$ & $5.17 \mathrm{E}-03$ & $5.17 \mathrm{E}-03$ & $5.17 \mathrm{E}-03$ & $5.17 \mathrm{E}-03$ & $5.17 \mathrm{E}-03$ & 5.17E-03 & $5.17 \mathrm{E}-03$ & 5.17E-03 & $5.17 \mathrm{E}-03$ \\
\hline MO 97 & $2.92 \mathrm{E}-03$ & $2.92 \mathrm{E}-03$ & $2.92 \mathrm{E}-03$ & $2.92 \mathrm{E}-03$ & $2.92 \mathrm{E}-03$ & $2.92 \mathrm{E}-03$ & $2.92 \mathrm{E}-03$ & $2.92 \mathrm{E}-03$ & $2.92 \mathrm{E}-03$ & $2.92 \mathrm{E}-03$ \\
\hline MO 98 & $7.38 \mathrm{E}-03$ & $7.38 \mathrm{E}-03$ & $7.38 \mathrm{E}-03$ & $7.38 \mathrm{E}-03$ & $7.38 \mathrm{E}-03$ & $7.38 \mathrm{E}-03$ & $7.38 \mathrm{E}-03$ & $7.38 \mathrm{E}-03$ & $7.38 \mathrm{E}-03$ & $7.38 \mathrm{E}-03$ \\
\hline MO 99 & $3.20 \mathrm{E}-07$ & $3.19 \mathrm{E}-07$ & $2.49 \mathrm{E}-07$ & $5.48 \mathrm{E}-08$ & 1.67E-10 & $8.66 \mathrm{E}-14$ & $4.50 \mathrm{E}-17$ & $2.34 \mathrm{E}-20$ & $4.10 \mathrm{E}-29$ & $0.00 \mathrm{E}+00$ \\
\hline
\end{tabular}


TEM-10200-1

$12 / 19 / 17$

Rev.08

Title: AS-RUN NEUTRONICS EVALUATION FOR THE GE HITACHI EXPERIMENT IN THE ATR ECAR NO.:

\section{ENGINEERING CALCULATIONS AND ANALYSIS}

Page $\mathbf{5 2}$ of $\mathbf{5 7}$

\begin{tabular}{|c|c|c|c|c|c|c|c|c|c|c|}
\hline \multicolumn{11}{|c|}{$1 \mathrm{~g}$ of $316 \mathrm{SS}$} \\
\hline Jsetone & EOI & $30.0 \mathrm{MI}$ & $1.0 \mathrm{D}$ & $7.0 \mathrm{D}$ & $30.0 \mathrm{D}$ & $60.0 \mathrm{D}$ & $90.0 \mathrm{D}$ & $120.0 \mathrm{D}$ & $200.0 \mathrm{D}$ & $360.0 \mathrm{D}$ \\
\hline tsolupe & $\mathrm{g} / 1 \mathrm{~g}$ of $316 \mathrm{SS}$ & $\mathrm{g} / 1 \mathrm{~g}$ of $316 \mathrm{SS}$ & $\mathrm{g} / 1 \mathrm{~g}$ of $316 \mathrm{SS}$ & $\mathrm{g} / 1 \mathrm{~g}$ of $316 \mathrm{SS}$ & $\mathrm{g} / 1 \mathrm{~g}$ of $316 \mathrm{SS}$ & $\mathrm{g} / 1 \mathrm{~g}$ of $316 \mathrm{SS}$ & $\mathrm{g} / 1 \mathrm{~g}$ of $316 \mathrm{SS}$ & $\mathrm{g} / 1 \mathrm{~g}$ of $316 \mathrm{SS}$ & $\mathrm{g} / 1 \mathrm{~g}$ of $316 \mathrm{SS}$ & $\mathrm{g} / 1 \mathrm{~g}$ of $316 \mathrm{SS}$ \\
\hline M0100 & $3.00 \mathrm{E}-03$ & $3.00 \mathrm{E}-03$ & $3.00 \mathrm{E}-03$ & $3.00 \mathrm{E}-03$ & $3.00 \mathrm{E}-03$ & $3.00 \mathrm{E}-03$ & $3.00 \mathrm{E}-03$ & $3.00 \mathrm{E}-03$ & $3.00 \mathrm{E}-03$ & $3.00 \mathrm{E}-03$ \\
\hline M0101 & $3.00 \mathrm{E}-10$ & $7.23 \mathrm{E}-11$ & $0.00 \mathrm{E}+00$ & $0.00 \mathrm{E}+00$ & $0.00 \mathrm{E}+00$ & $0.00 \mathrm{E}+00$ & $0.00 \mathrm{E}+00$ & $0.00 \mathrm{E}+00$ & $0.00 \mathrm{E}+00$ & $0.00 \mathrm{E}+00$ \\
\hline TC 99 & $2.07 \mathrm{E}-06$ & 2.07E-06 & $2.08 \mathrm{E}-06$ & $2.10 \mathrm{E}-06$ & $2.11 \mathrm{E}-06$ & $2.11 \mathrm{E}-06$ & $2.11 \mathrm{E}-06$ & $2.11 \mathrm{E}-06$ & $2.11 \mathrm{E}-06$ & $2.11 \mathrm{E}-06$ \\
\hline TC100 & $2.30 \mathrm{E}-13$ & $0.00 \mathrm{E}+00$ & $0.00 \mathrm{E}+00$ & $0.00 \mathrm{E}+00$ & $0.00 \mathrm{E}+00$ & $0.00 \mathrm{E}+00$ & $0.00 \mathrm{E}+00$ & $0.00 \mathrm{E}+00$ & $0.00 \mathrm{E}+00$ & $0.00 \mathrm{E}+00$ \\
\hline TC101 & $2.91 \mathrm{E}-10$ & $1.68 \mathrm{E}-10$ & $0.00 \mathrm{E}+00$ & $0.00 \mathrm{E}+00$ & $0.00 \mathrm{E}+00$ & $0.00 \mathrm{E}+00$ & $0.00 \mathrm{E}+00$ & $0.00 \mathrm{E}+00$ & $0.00 \mathrm{E}+00$ & $0.00 \mathrm{E}+00$ \\
\hline RU 99 & $1.99 \mathrm{E}-12$ & $1.99 \mathrm{E}-12$ & $2.01 \mathrm{E}-12$ & $2.12 \mathrm{E}-12$ & $2.55 \mathrm{E}-12$ & $3.11 \mathrm{E}-12$ & $3.68 \mathrm{E}-12$ & $4.24 \mathrm{E}-12$ & $5.75 \mathrm{E}-12$ & $8.75 \mathrm{E}-12$ \\
\hline RU100 & $9.51 \mathrm{E}-08$ & $9.51 \mathrm{E}-08$ & $9.51 \mathrm{E}-08$ & $9.51 \mathrm{E}-08$ & $9.51 \mathrm{E}-08$ & $9.51 \mathrm{E}-08$ & $9.51 \mathrm{E}-08$ & $9.51 \mathrm{E}-08$ & $9.51 \mathrm{E}-08$ & $9.51 \mathrm{E}-08$ \\
\hline RU101 & $4.43 \mathrm{E}-06$ & $4.43 \mathrm{E}-06$ & $4.43 \mathrm{E}-06$ & $4.43 \mathrm{E}-06$ & $4.43 \mathrm{E}-06$ & $4.43 \mathrm{E}-06$ & $4.43 \mathrm{E}-06$ & $4.43 \mathrm{E}-06$ & $4.43 \mathrm{E}-06$ & $4.43 \mathrm{E}-06$ \\
\hline RU102 & $6.64 \mathrm{E}-08$ & $6.64 \mathrm{E}-08$ & $6.64 \mathrm{E}-08$ & $6.64 \mathrm{E}-08$ & $6.64 \mathrm{E}-08$ & $6.64 \mathrm{E}-08$ & $6.64 \mathrm{E}-08$ & $6.64 \mathrm{E}-08$ & $6.64 \mathrm{E}-08$ & $6.64 \mathrm{E}-08$ \\
\hline RU103 & $2.79 \mathrm{E}-11$ & $2.78 \mathrm{E}-11$ & $2.74 \mathrm{E}-11$ & $2.46 \mathrm{E}-11$ & $1.64 \mathrm{E}-11$ & $9.66 \mathrm{E}-12$ & $5.69 \mathrm{E}-12$ & $3.35 \mathrm{E}-12$ & $8.17 \mathrm{E}-13$ & $4.85 \mathrm{E}-14$ \\
\hline RU104 & $2.15 \mathrm{E}-13$ & $2.15 \mathrm{E}-13$ & $2.15 \mathrm{E}-13$ & $2.15 \mathrm{E}-13$ & $2.15 \mathrm{E}-13$ & $2.15 \mathrm{E}-13$ & $2.15 \mathrm{E}-13$ & $2.15 \mathrm{E}-13$ & $2.15 \mathrm{E}-13$ & $2.15 \mathrm{E}-13$ \\
\hline RH103 & $2.81 \mathrm{E}-12$ & $2.81 \mathrm{E}-12$ & $2.86 \mathrm{E}-12$ & $3.13 \mathrm{E}-12$ & $3.95 \mathrm{E}-12$ & $4.62 \mathrm{E}-12$ & $5.01 \mathrm{E}-12$ & $5.24 \mathrm{E}-12$ & $5.50 \mathrm{E}-12$ & $5.57 \mathrm{E}-12$ \\
\hline PD104 & $2.49 \mathrm{E}-13$ & $2.49 \mathrm{E}-13$ & $2.49 \mathrm{E}-13$ & $2.49 \mathrm{E}-13$ & $2.49 \mathrm{E}-13$ & $2.49 \mathrm{E}-13$ & $2.49 \mathrm{E}-13$ & $2.49 \mathrm{E}-13$ & $2.49 \mathrm{E}-13$ & $2.49 \mathrm{E}-13$ \\
\hline SUMTOT & $1.00 \mathrm{E}+00$ & $1.00 \mathrm{E}+00$ & $1.00 \mathrm{E}+00$ & $1.00 \mathrm{E}+00$ & $1.00 \mathrm{E}+00$ & $1.00 \mathrm{E}+00$ & $1.00 \mathrm{E}+00$ & $1.00 \mathrm{E}+00$ & $1.00 \mathrm{E}+00$ & $1.00 \mathrm{E}+00$ \\
\hline OTOTAL & $1.00 \mathrm{E}+00$ & $1.00 \mathrm{E}+00$ & $1.00 \mathrm{E}+00$ & $1.00 \mathrm{E}+00$ & $1.00 \mathrm{E}+00$ & $1.00 \mathrm{E}+00$ & $1.00 \mathrm{E}+00$ & $1.00 \mathrm{E}+00$ & $1.00 \mathrm{E}+00$ & $1.00 \mathrm{E}+00$ \\
\hline
\end{tabular}

Table 21. $\mathrm{g} / 1 \mathrm{~g}$ of $\mathrm{Ti}$

\begin{tabular}{|c|c|c|c|c|c|c|c|c|c|c|}
\hline \multicolumn{11}{|c|}{$1 \mathrm{~g}$ of $\mathrm{Ti}$} \\
\hline & EOI & $30.0 \mathrm{MI}$ & $1.0 \mathrm{D}$ & $7.0 \mathrm{D}$ & $30.0 \mathrm{D}$ & $60.0 \mathrm{D}$ & $90.0 \mathrm{D}$ & $120.0 \mathrm{D}$ & $200.0 \mathrm{D}$ & $360.0 \mathrm{D}$ \\
\hline Isotope & $\mathrm{g} / 1 \mathrm{~g}$ of $\mathrm{Ti}$ & $\mathrm{g} / 1 \mathrm{~g}$ of $\mathrm{Ti}$ & $\mathrm{g} / 1 \mathrm{~g}$ of $\mathrm{Ti}$ & $\mathrm{g} / 1 \mathrm{~g}$ of $\mathrm{Ti}$ & $\mathrm{g} / 1 \mathrm{~g}$ of $\mathrm{Ti}$ & $\mathrm{g} / 1 \mathrm{~g}$ of $\mathrm{Ti}$ & $\mathrm{g} / 1 \mathrm{~g}$ of $\mathrm{Ti}$ & $\mathrm{g} / 1 \mathrm{~g}$ of $\mathrm{Ti}$ & $\mathrm{g} / 1 \mathrm{~g}$ of $\mathrm{Ti}$ & $\mathrm{g} / 1 \mathrm{~g}$ of $\mathrm{T}$ \\
\hline H 1 & $1.91 \mathrm{E}-07$ & $1.91 \mathrm{E}-07$ & $1.91 \mathrm{E}-07$ & $1.91 \mathrm{E}-07$ & $1.91 \mathrm{E}-07$ & $1.91 \mathrm{E}-07$ & $1.91 \mathrm{E}-07$ & $1.91 \mathrm{E}-07$ & $1.91 \mathrm{E}-07$ & $1.91 \mathrm{E}-07$ \\
\hline $\mathrm{H} 2$ & $6.65 \mathrm{E}-11$ & $6.65 \mathrm{E}-11$ & $6.65 \mathrm{E}-11$ & $6.65 \mathrm{E}-11$ & $6.65 \mathrm{E}-11$ & $6.65 \mathrm{E}-11$ & $6.65 \mathrm{E}-11$ & $6.65 \mathrm{E}-11$ & $6.65 \mathrm{E}-11$ & $6.65 \mathrm{E}-11$ \\
\hline HE 4 & $1.33 \mathrm{E}-07$ & $1.33 \mathrm{E}-07$ & $1.33 \mathrm{E}-07$ & $1.33 \mathrm{E}-07$ & $1.33 \mathrm{E}-07$ & $1.33 \mathrm{E}-07$ & $1.33 \mathrm{E}-07$ & $1.33 \mathrm{E}-07$ & $1.33 \mathrm{E}-07$ & $1.33 \mathrm{E}-07$ \\
\hline AR 40 & $1.80 \mathrm{E}-12$ & $1.80 \mathrm{E}-12$ & $1.80 \mathrm{E}-12$ & $1.80 \mathrm{E}-12$ & $1.80 \mathrm{E}-12$ & $1.80 \mathrm{E}-12$ & $1.80 \mathrm{E}-12$ & $1.80 \mathrm{E}-12$ & $1.80 \mathrm{E}-12$ & $1.80 \mathrm{E}-12$ \\
\hline K 41 & $5.81 \mathrm{E}-14$ & $5.81 \mathrm{E}-14$ & $5.82 \mathrm{E}-14$ & $5.82 \mathrm{E}-14$ & $5.82 \mathrm{E}-14$ & $5.82 \mathrm{E}-14$ & $5.82 \mathrm{E}-14$ & $5.82 \mathrm{E}-14$ & $5.82 \mathrm{E}-14$ & $5.82 \mathrm{E}-14$ \\
\hline K 43 & $9.61 \mathrm{E}-15$ & 9.47E-15 & $4.60 \mathrm{E}-15$ & $5.56 \mathrm{E}-17$ & $2.47 \mathrm{E}-24$ & $6.34 \mathrm{E}-34$ & $1.81 \mathrm{E}-43$ & $0.00 E+00$ & $0.00 \mathrm{E}+00$ & $0.00 \mathrm{E}+0 \mathrm{C}$ \\
\hline CA 43 & $1.50 \mathrm{E}-07$ & $1.50 \mathrm{E}-07$ & $1.50 \mathrm{E}-07$ & $1.50 \mathrm{E}-07$ & $1.50 \mathrm{E}-07$ & $1.50 \mathrm{E}-07$ & $1.50 \mathrm{E}-07$ & $1.50 \mathrm{E}-07$ & $1.50 \mathrm{E}-07$ & $1.50 \mathrm{E}-07$ \\
\hline CA 44 & $1.16 \mathrm{E}-06$ & $1.16 \mathrm{E}-06$ & $1.16 \mathrm{E}-06$ & $1.16 \mathrm{E}-06$ & $1.16 \mathrm{E}-06$ & $1.16 \mathrm{E}-06$ & $1.16 \mathrm{E}-06$ & $1.16 \mathrm{E}-06$ & $1.16 \mathrm{E}-06$ & $1.16 \mathrm{E}-06$ \\
\hline CA 45 & $1.56 \mathrm{E}-08$ & $1.56 \mathrm{E}-08$ & $1.56 \mathrm{E}-08$ & $1.52 \mathrm{E}-08$ & $1.38 \mathrm{E}-08$ & $1.21 \mathrm{E}-08$ & 1.07E-08 & $9.38 \mathrm{E}-09$ & $6.67 \mathrm{E}-09$ & $3.38 \mathrm{E}-09$ \\
\hline CA 46 & $1.35 \mathrm{E}-07$ & $1.35 \mathrm{E}-07$ & $1.35 \mathrm{E}-07$ & $1.35 \mathrm{E}-07$ & $1.35 \mathrm{E}-07$ & $1.35 \mathrm{E}-07$ & $1.35 \mathrm{E}-07$ & $1.35 \mathrm{E}-07$ & $1.35 \mathrm{E}-07$ & $1.35 \mathrm{E}-07$ \\
\hline CA 47 & $4.46 \mathrm{E}-12$ & $4.45 \mathrm{E}-12$ & $3.83 \mathrm{E}-12$ & $1.53 \mathrm{E}-12$ & $4.55 \mathrm{E}-14$ & $4.65 \mathrm{E}-16$ & $4.75 \mathrm{E}-18$ & $4.84 \mathrm{E}-20$ & $2.38 \mathrm{E}-25$ & $5.72 \mathrm{E}-36$ \\
\hline SC 45 & $8.26 \mathrm{E}-09$ & $8.26 \mathrm{E}-09$ & $8.33 \mathrm{E}-09$ & $8.72 \mathrm{E}-09$ & $1.01 \mathrm{E}-08$ & $1.18 \mathrm{E}-08$ & $1.32 \mathrm{E}-08$ & $1.45 \mathrm{E}-08$ & $1.72 \mathrm{E}-08$ & $2.05 \mathrm{E}-08$ \\
\hline SC 46 & $1.44 \mathrm{E}-06$ & $1.44 \mathrm{E}-06$ & $1.42 \mathrm{E}-06$ & $1.36 \mathrm{E}-06$ & $1.12 \mathrm{E}-06$ & $8.74 \mathrm{E}-07$ & $6.82 \mathrm{E}-07$ & $5.32 \mathrm{E}-07$ & $2.75 \mathrm{E}-07$ & $7.31 \mathrm{E}-08$ \\
\hline SC 47 & $1.09 \mathrm{E}-07$ & $1.08 \mathrm{E}-07$ & $8.85 \mathrm{E}-08$ & $2.56 \mathrm{E}-08$ & $2.20 \mathrm{E}-10$ & $4.45 \mathrm{E}-13$ & $9.06 \mathrm{E}-16$ & $1.94 \mathrm{E}-18$ & $7.89 \mathrm{E}-25$ & $1.62 \mathrm{E}-35$ \\
\hline
\end{tabular}


TEM-10200-1

$12 / 19 / 17$

Rev.08

Title:

AS-RUN NEUTRONICS EVALUATION FOR THE GE HITACHI EXPERIMENT IN THE ATR

\begin{tabular}{|c|c|c|c|c|c|c|c|c|c|c|}
\hline \multicolumn{11}{|c|}{$1 \mathrm{~g}$ of $\mathrm{Ti}$} \\
\hline Isotono & EOI & $30.0 \mathrm{MI}$ & $1.0 \mathrm{D}$ & $7.0 \mathrm{D}$ & $30.0 \mathrm{D}$ & $60.0 \mathrm{D}$ & $90.0 \mathrm{D}$ & $120.0 \mathrm{D}$ & $200.0 \mathrm{D}$ & $360.0 \mathrm{D}$ \\
\hline Isotope & $\mathrm{g} / 1 \mathrm{~g}$ of $\mathrm{Ti}$ & $\mathrm{g} / 1 \mathrm{~g}$ of $\mathrm{Ti}$ & $\mathrm{g} / 1 \mathrm{~g}$ of $\mathrm{Ti}$ & $\mathrm{g} / 1 \mathrm{~g}$ of $\mathrm{Ti}$ & $\mathrm{g} / 1 \mathrm{~g}$ of $\mathrm{Ti}$ & $\mathrm{g} / 1 \mathrm{~g}$ of $\mathrm{Ti}$ & $\mathrm{g} / 1 \mathrm{~g}$ of $\mathrm{Ti}$ & $\mathrm{g} / 1 \mathrm{~g}$ of $\mathrm{Ti}$ & $\mathrm{g} / 1 \mathrm{~g}$ of $\mathrm{Ti}$ & $g / 1 g$ of $T$ \\
\hline SC 48 & 5.67E-09 & $5.63 \mathrm{E}-09$ & $3.88 \mathrm{E}-09$ & $3.97 \mathrm{E}-10$ & $6.39 \mathrm{E}-14$ & $7.21 \mathrm{E}-19$ & $8.13 \mathrm{E}-24$ & $9.17 \mathrm{E}-29$ & $5.85 \mathrm{E}-42$ & $0.00 E+0 C$ \\
\hline SC 49 & $1.12 \mathrm{E}-10$ & $7.82 \mathrm{E}-11$ & $3.25 \mathrm{E}-18$ & $0.00 \mathrm{E}+00$ & $0.00 \mathrm{E}+00$ & $0.00 \mathrm{E}+00$ & $0.00 \mathrm{E}+00$ & $0.00 \mathrm{E}+00$ & $0.00 \mathrm{E}+00$ & $0.00 E+0 C$ \\
\hline SC 50 & $\begin{array}{l}6.51 \mathrm{E}-14 \\
\end{array}$ & 3.37E-19 & $0.00 \mathrm{E}+00$ & $0.00 \mathrm{E}+00$ & $0.00 \mathrm{E}+00$ & $0.00 \mathrm{E}+00$ & $0.00 \mathrm{E}+00$ & $0.00 \mathrm{E}+00$ & $0.00 \mathrm{E}+00$ & $0.00 \mathrm{E}+0 \mathrm{C}$ \\
\hline TI 46 & $7.92 \mathrm{E}-02$ & $7.92 \mathrm{E}-02$ & $7.92 \mathrm{E}-02$ & $7.92 \mathrm{E}-02$ & $7.92 \mathrm{E}-02$ & $7.92 \mathrm{E}-02$ & $7.92 \mathrm{E}-02$ & $7.92 \mathrm{E}-02$ & $7.92 \mathrm{E}-02$ & $7.92 \mathrm{E}-02$ \\
\hline TI 47 & $7.30 \mathrm{E}-02$ & $7.30 \mathrm{E}-02$ & $7.30 \mathrm{E}-02$ & $7.30 \mathrm{E}-02$ & $\begin{array}{l}7.30 \mathrm{E}-02 \\
\end{array}$ & $7.30 \mathrm{E}-02$ & $7.30 \mathrm{E}-02$ & $\begin{array}{l}7.30 \mathrm{E}-02 \\
\end{array}$ & $7.30 \mathrm{E}-02$ & $7.30 \mathrm{E}-02$ \\
\hline TI 48 & $7.33 \mathrm{E}-01$ & $7.33 \mathrm{E}-01$ & $7.33 \mathrm{E}-01$ & $7.33 \mathrm{E}-01$ & $7.33 \mathrm{E}-01$ & $7.33 \mathrm{E}-01$ & $7.33 \mathrm{E}-01$ & $7.33 \mathrm{E}-01$ & $7.33 \mathrm{E}-01$ & $7.33 \mathrm{E}-01$ \\
\hline TI 49 & $6.05 \mathrm{E}-02$ & $6.05 \mathrm{E}-02$ & $6.05 \mathrm{E}-02$ & $6.05 \mathrm{E}-02$ & $6.05 \mathrm{E}-02$ & $6.05 \mathrm{E}-02$ & $6.05 \mathrm{E}-02$ & $6.05 \mathrm{E}-02$ & $6.05 \mathrm{E}-02$ & $6.05 \mathrm{E}-02$ \\
\hline TI 50 & $5.44 \mathrm{E}-02$ & $5.44 \mathrm{E}-02$ & $5.44 \mathrm{E}-02$ & $5.44 \mathrm{E}-02$ & $5.44 \mathrm{E}-02$ & $5.44 \mathrm{E}-02$ & 5.44E-02 & $5.44 \mathrm{E}-02$ & $5.44 \mathrm{E}-02$ & $5.44 \mathrm{E}-02$ \\
\hline TI 51 & $2.54 \mathrm{E}-10$ & $6.87 \mathrm{E}-12$ & $0.00 \mathrm{E}+00$ & $0.00 \mathrm{E}+00$ & $0.00 \mathrm{E}+00$ & $0.00 \mathrm{E}+00$ & $0.00 \mathrm{E}+00$ & $0.00 \mathrm{E}+00$ & $0.00 \mathrm{E}+00$ & $0.00 \mathrm{E}+0 \mathrm{C}$ \\
\hline V51 & $9.62 \mathrm{E}-06$ & $9.62 \mathrm{E}-06$ & $9.62 \mathrm{E}-06$ & $9.62 \mathrm{E}-06$ & $9.62 \mathrm{E}-06$ & $9.62 \mathrm{E}-06$ & $9.62 \mathrm{E}-06$ & $9.62 \mathrm{E}-06$ & $9.62 \mathrm{E}-06$ & $9.62 \mathrm{E}-06$ \\
\hline V 52 & $7.76 \mathrm{E}-13$ & $3.03 \mathrm{E}-15$ & $0.00 \mathrm{E}+00$ & $0.00 \mathrm{E}+00$ & $0.00 \mathrm{E}+00$ & $0.00 \mathrm{E}+00$ & $0.00 \mathrm{E}+00$ & $0.00 \mathrm{E}+00$ & $0.00 \mathrm{E}+00$ & $0.00 \mathrm{E}+0 \mathrm{C}$ \\
\hline CR 52 & $2.26 \mathrm{E}-08$ & 2.27E-08 & 2.27E-08 & 2.27E-08 & 2.27E-08 & 2.27E-08 & 2.27E-08 & 2.27E-08 & 2.27E-08 & $2.27 \mathrm{E}-08$ \\
\hline CR 53 & $2.57 \mathrm{E}-11$ & $2.57 \mathrm{E}-11$ & $2.57 \mathrm{E}-11$ & $2.57 \mathrm{E}-11$ & $2.57 \mathrm{E}-11$ & $2.57 \mathrm{E}-11$ & $2.57 \mathrm{E}-11$ & $2.57 \mathrm{E}-11$ & $2.57 \mathrm{E}-11$ & $2.57 \mathrm{E}-11$ \\
\hline CR 54 & $1.11 \mathrm{E}-13$ & $1.11 \mathrm{E}-13$ & $1.11 \mathrm{E}-13$ & $1.11 \mathrm{E}-13$ & $1.11 \mathrm{E}-13$ & $1.11 \mathrm{E}-13$ & $1.11 \mathrm{E}-13$ & $1.11 \mathrm{E}-13$ & $1.11 \mathrm{E}-13$ & $1.11 \mathrm{E}-13$ \\
\hline SUMTOT & $1.00 \mathrm{E}+00$ & $1.00 \mathrm{E}+00$ & $1.00 \mathrm{E}+00$ & $1.00 \mathrm{E}+00$ & $1.00 \mathrm{E}+00$ & $1.00 \mathrm{E}+00$ & $1.00 \mathrm{E}+00$ & $1.00 \mathrm{E}+00$ & $1.00 \mathrm{E}+00$ & $1.00 \mathrm{E}+0 \mathrm{C}$ \\
\hline OTOTAL & $1.00 \mathrm{E}+00$ & $1.00 \mathrm{E}+00$ & $1.00 \mathrm{E}+00$ & $1.00 \mathrm{E}+00$ & $1.00 \mathrm{E}+00$ & $1.00 \mathrm{E}+00$ & $1.00 \mathrm{E}+00$ & $1.00 \mathrm{E}+00$ & $1.00 \mathrm{E}+00$ & $1.00 \mathrm{E}+0 \mathrm{C}$ \\
\hline
\end{tabular}

Page $\mathbf{5 3}$ of $\mathbf{5 7}$ PROJECT NO.: 32535 Date: 12/02/2019

Table 22. Ci/1g of Ti

\begin{tabular}{|c|c|c|c|c|c|c|c|c|c|c|}
\hline \multicolumn{11}{|c|}{$1 \mathrm{~g}$ of $\mathrm{Ti}$} \\
\hline \multirow{2}{*}{ Isotope } & EOI & $30.0 \mathrm{MI}$ & $1.0 \mathrm{D}$ & $7.0 \mathrm{D}$ & $30.0 \mathrm{D}$ & $60.0 \mathrm{D}$ & $90.0 \mathrm{D}$ & $120.0 \mathrm{D}$ & $200.0 \mathrm{D}$ & $360.0 \mathrm{D}$ \\
\hline & $\mathrm{Ci} / 1 \mathrm{~g}$ of $\mathrm{Ti}$ & $\mathrm{Ci} / 1 \mathrm{~g}$ of $\mathrm{Ti}$ & $\mathrm{Ci} / 1 \mathrm{~g}$ of $\mathrm{Ti}$ & $\mathrm{Ci} / 1 \mathrm{~g}$ of $\mathrm{Ti}$ & $\mathrm{Ci} / 1 \mathrm{~g}$ of $\mathrm{Ti}$ & $\mathrm{Ci} / 1 \mathrm{~g}$ of $\mathrm{Ti}$ & $\mathrm{Ci} / 1 \mathrm{~g}$ of $\mathrm{Ti}$ & $\mathrm{Ci} / 1 \mathrm{~g}$ of $\mathrm{Ti}$ & $\mathrm{Ci} / 1 \mathrm{~g}$ of $\mathrm{Ti}$ & $\mathrm{Ci} / 1 \mathrm{~g}$ of $\mathrm{Ti}$ \\
\hline H 3 & $1.56 \mathrm{E}-13$ & $1.56 \mathrm{E}-13$ & $1.56 \mathrm{E}-13$ & $1.56 \mathrm{E}-13$ & $1.55 \mathrm{E}-13$ & $1.55 \mathrm{E}-13$ & $1.54 \mathrm{E}-13$ & $1.53 \mathrm{E}-13$ & $1.51 \mathrm{E}-13$ & $1.48 \mathrm{E}-13$ \\
\hline S37 & $2.24 \mathrm{E}-11$ & $3.69 \mathrm{E}-13$ & $0.00 \mathrm{E}+00$ & $0.00 \mathrm{E}+00$ & $0.00 \mathrm{E}+00$ & $0.00 \mathrm{E}+00$ & $0.00 \mathrm{E}+00$ & $0.00 \mathrm{E}+00$ & $0.00 \mathrm{E}+00$ & $0.00 \mathrm{E}+00$ \\
\hline CL 38 & $3.16 \mathrm{E}-15$ & $1.40 \mathrm{E}-15$ & $5.50 \mathrm{E}-27$ & $0.00 \mathrm{E}+00$ & $0.00 \mathrm{E}+00$ & $0.00 \mathrm{E}+00$ & $0.00 \mathrm{E}+00$ & $0.00 \mathrm{E}+00$ & $0.00 \mathrm{E}+00$ & $0.00 \mathrm{E}+00$ \\
\hline AR 41 & $2.45 \mathrm{E}-09$ & $2.02 \mathrm{E}-09$ & $2.71 \mathrm{E}-13$ & $0.00 \mathrm{E}+00$ & $0.00 \mathrm{E}+00$ & $0.00 \mathrm{E}+00$ & $0.00 \mathrm{E}+00$ & $0.00 \mathrm{E}+00$ & $0.00 \mathrm{E}+00$ & $0.00 \mathrm{E}+00$ \\
\hline AR 42 & $3.53 \mathrm{E}-18$ & $3.53 \mathrm{E}-18$ & $3.53 \mathrm{E}-18$ & $3.53 \mathrm{E}-18$ & $3.52 \mathrm{E}-18$ & $3.52 \mathrm{E}-18$ & $3.51 \mathrm{E}-18$ & $3.50 \mathrm{E}-18$ & $3.49 \mathrm{E}-18$ & 3.46E-18 \\
\hline K 42 & 1.99E-11 & $1.93 \mathrm{E}-11$ & $5.18 \mathrm{E}-12$ & $1.62 \mathrm{E}-15$ & $3.52 \mathrm{E}-18$ & $3.52 \mathrm{E}-18$ & $3.51 \mathrm{E}-18$ & 3.50E-18 & 3.49E-18 & 3.46E-18 \\
\hline K 43 & $3.10 \mathrm{E}-08$ & 3.05E-08 & 1.49E-08 & $1.79 \mathrm{E}-10$ & $7.96 \mathrm{E}-18$ & $2.05 \mathrm{E}-27$ & $0.00 \mathrm{E}+00$ & $0.00 \mathrm{E}+00$ & $0.00 \mathrm{E}+00$ & $0.00 \mathrm{E}+00$ \\
\hline K 44 & $5.53 \mathrm{E}-09$ & 2.15E-09 & 1.09E-28 & $0.00 \mathrm{E}+00$ & $0.00 \mathrm{E}+00$ & $0.00 \mathrm{E}+00$ & $0.00 \mathrm{E}+00$ & $0.00 \mathrm{E}+00$ & $0.00 \mathrm{E}+00$ & $0.00 \mathrm{E}+00$ \\
\hline CA 45 & $2.78 \mathrm{E}-04$ & $2.78 \mathrm{E}-04$ & $2.77 \mathrm{E}-04$ & $2.70 \mathrm{E}-04$ & $2.45 \mathrm{E}-04$ & $2.16 \mathrm{E}-04$ & 1.90E-04 & 1.67E-04 & 1.19E-04 & $6.02 \mathrm{E}-05$ \\
\hline CA 47 & $2.73 \mathrm{E}-06$ & $2.72 \mathrm{E}-06$ & 2.35E-06 & $9.38 \mathrm{E}-07$ & $2.79 \mathrm{E}-08$ & $2.85 \mathrm{E}-10$ & $2.91 \mathrm{E}-12$ & 2.97E-14 & 1.46E-19 & $3.51 \mathrm{E}-30$ \\
\hline SC 46 & $4.87 \mathrm{E}-02$ & $4.86 \mathrm{E}-02$ & $4.83 \mathrm{E}-02$ & $4.59 \mathrm{E}-02$ & $3.80 \mathrm{E}-02$ & $2.96 \mathrm{E}-02$ & $2.31 \mathrm{E}-02$ & $1.80 \mathrm{E}-02$ & $9.30 \mathrm{E}-03$ & $2.48 \mathrm{E}-03$ \\
\hline
\end{tabular}


TEM-10200-1

$12 / 19 / 17$

Rev.08

Title:

AS-RUN NEUTRONICS EVALUATION FOR THE GE HITACHI EXPERIMENT IN THE ATR

\begin{tabular}{llllllll} 
ECAR NO.: & 4740 & REV. NO.: & 0 & PROJECT NO.: & 32535 & Date: & $12 / 02 / 2019$ \\
\hline
\end{tabular}

\begin{tabular}{|c|c|c|c|c|c|c|c|c|c|c|}
\hline \multicolumn{11}{|c|}{$1 \mathrm{~g}$ of $\mathrm{Ti}$} \\
\hline \multirow{2}{*}{ Isotope } & EOI & $30.0 \mathrm{MI}$ & $1.0 \mathrm{D}$ & $7.0 \mathrm{D}$ & $30.0 \mathrm{D}$ & $60.0 \mathrm{D}$ & $90.0 \mathrm{D}$ & $120.0 \mathrm{D}$ & $200.0 \mathrm{D}$ & $360.0 \mathrm{D}$ \\
\hline & $\mathrm{Ci} / 1 \mathrm{~g}$ of $\mathrm{Ti}$ & $\mathrm{Ci} / 1 \mathrm{~g}$ of $\mathrm{Ti}$ & $\mathrm{Ci} / 1 \mathrm{~g}$ of $\mathrm{Ti}$ & $\mathrm{Ci} / 1 \mathrm{~g}$ of $\mathrm{Ti}$ & $\mathrm{Ci} / 1 \mathrm{~g}$ of $\mathrm{Ti}$ & $\mathrm{Ci} / 1 \mathrm{~g}$ of $\mathrm{Ti}$ & $\mathrm{Ci} / 1 \mathrm{~g}$ of $\mathrm{Ti}$ & $\mathrm{Ci} / 1 \mathrm{~g}$ of $\mathrm{Ti}$ & $\mathrm{Ci} / 1 \mathrm{~g}$ of $\mathrm{Ti}$ & $\mathrm{Ci} / 1 \mathrm{~g}$ of $\mathrm{Ti}$ \\
\hline SC 46M & $1.39 \mathrm{E}-06$ & $0.00 \mathrm{E}+00$ & $0.00 \mathrm{E}+00$ & $0.00 \mathrm{E}+00$ & $0.00 \mathrm{E}+00$ & $0.00 \mathrm{E}+00$ & $0.00 \mathrm{E}+00$ & $0.00 \mathrm{E}+00$ & $0.00 \mathrm{E}+00$ & $0.00 \mathrm{E}+00$ \\
\hline SC 47 & $9.03 E-02$ & 8.99E-02 & $7.34 \mathrm{E}-02$ & $2.12 \mathrm{E}-02$ & $1.82 \mathrm{E}-04$ & $3.69 \mathrm{E}-07$ & $7.52 \mathrm{E}-10$ & $1.61 \mathrm{E}-12$ & $6.54 \mathrm{E}-19$ & $1.35 \mathrm{E}-29$ \\
\hline SC 48 & $8.45 \mathrm{E}-03$ & $8.39 \mathrm{E}-03$ & $5.78 \mathrm{E}-03$ & $5.92 \mathrm{E}-04$ & $9.53 \mathrm{E}-08$ & $1.08 \mathrm{E}-12$ & $1.21 \mathrm{E}-17$ & $1.37 \mathrm{E}-22$ & $0.00 \mathrm{E}+00$ & $0.00 \mathrm{E}+00$ \\
\hline SC 49 & $7.50 \mathrm{E}-03$ & $5.22 \mathrm{E}-03$ & $2.17 \mathrm{E}-10$ & $0.00 \mathrm{E}+00$ & $0.00 \mathrm{E}+00$ & $0.00 \mathrm{E}+00$ & $0.00 \mathrm{E}+00$ & $0.00 \mathrm{E}+00$ & $0.00 \mathrm{E}+00$ & $0.00 E+00$ \\
\hline SC 50 & $1.43 \mathrm{E}-04$ & $7.41 \mathrm{E}-10$ & $0.00 \mathrm{E}+00$ & $0.00 \mathrm{E}+00$ & $0.00 \mathrm{E}+00$ & $0.00 \mathrm{E}+00$ & $0.00 \mathrm{E}+00$ & $0.00 \mathrm{E}+00$ & $0.00 \mathrm{E}+00$ & $0.00 \mathrm{E}+00$ \\
\hline TI 51 & $1.63 \mathrm{E}-01$ & $4.40 \mathrm{E}-03$ & $0.00 \mathrm{E}+00$ & $0.00 \mathrm{E}+00$ & $0.00 \mathrm{E}+00$ & $0.00 \mathrm{E}+00$ & $0.00 \mathrm{E}+00$ & $0.00 \mathrm{E}+00$ & $0.00 \mathrm{E}+00$ & $0.00 \mathrm{E}+00$ \\
\hline V 52 & $7.49 \mathrm{E}-04$ & $2.92 \mathrm{E}-06$ & $0.00 \mathrm{E}+00$ & $0.00 \mathrm{E}+00$ & $0.00 \mathrm{E}+00$ & $0.00 \mathrm{E}+00$ & $0.00 \mathrm{E}+00$ & $0.00 \mathrm{E}+00$ & $0.00 \mathrm{E}+00$ & $0.00 \mathrm{E}+00$ \\
\hline V53 & $1.27 \mathrm{E}-13$ & $3.12 \mathrm{E}-19$ & $0.00 \mathrm{E}+00$ & $0.00 \mathrm{E}+00$ & $0.00 \mathrm{E}+00$ & $0.00 \mathrm{E}+00$ & $0.00 \mathrm{E}+00$ & $0.00 \mathrm{E}+00$ & $0.00 \mathrm{E}+00$ & $0.00 \mathrm{E}+00$ \\
\hline CR 51 & $7.87 \mathrm{E}-11$ & $7.87 \mathrm{E}-11$ & $7.68 \mathrm{E}-11$ & $6.61 \mathrm{E}-11$ & $3.72 \mathrm{E}-11$ & $1.76 \mathrm{E}-11$ & $8.29 \mathrm{E}-12$ & $3.91 \mathrm{E}-12$ & $5.29 \mathrm{E}-13$ & $9.66 \mathrm{E}-15$ \\
\hline CR 55 & $5.93 \mathrm{E}-13$ & $3.15 \mathrm{E}-15$ & $0.00 \mathrm{E}+00$ & $0.00 \mathrm{E}+00$ & $0.00 \mathrm{E}+00$ & $0.00 \mathrm{E}+00$ & $0.00 \mathrm{E}+00$ & $0.00 \mathrm{E}+00$ & $0.00 \mathrm{E}+00$ & $0.00 \mathrm{E}+00$ \\
\hline MN 56 & $1.81 \mathrm{E}-15$ & $1.56 \mathrm{E}-15$ & $0.00 \mathrm{E}+00$ & $0.00 \mathrm{E}+00$ & $0.00 \mathrm{E}+00$ & $0.00 \mathrm{E}+00$ & $0.00 \mathrm{E}+00$ & $0.00 \mathrm{E}+00$ & $0.00 \mathrm{E}+00$ & $0.00 \mathrm{E}+00$ \\
\hline SUMTOT & $3.19 \mathrm{E}-01$ & $1.57 \mathrm{E}-01$ & $1.28 \mathrm{E}-01$ & $6.80 \mathrm{E}-02$ & $3.84 \mathrm{E}-02$ & $2.98 \mathrm{E}-02$ & $2.33 \mathrm{E}-02$ & $1.82 \mathrm{E}-02$ & $9.42 \mathrm{E}-03$ & $2.54 \mathrm{E}-03$ \\
\hline OTOTAL & $3.19 \mathrm{E}-01$ & $1.57 \mathrm{E}-01$ & $1.28 \mathrm{E}-01$ & $6.80 \mathrm{E}-02$ & $3.84 \mathrm{E}-02$ & $2.98 \mathrm{E}-02$ & $2.33 \mathrm{E}-02$ & $1.82 \mathrm{E}-02$ & $9.42 \mathrm{E}-03$ & $2.54 \mathrm{E}-03$ \\
\hline
\end{tabular}

Table 23. $\mathrm{g} / \mathrm{1g}$ of $\mathrm{Nb}$

\begin{tabular}{|c|c|c|c|c|c|c|c|c|c|c|}
\hline \multicolumn{11}{|c|}{$1 \mathrm{~g}$ of $\mathrm{Nb}$} \\
\hline & EOI & $30.0 \mathrm{Ml}$ & $1.0 \mathrm{D}$ & $7.0 \mathrm{D}$ & $30.0 \mathrm{D}$ & $60.0 \mathrm{D}$ & $90.0 \mathrm{D}$ & $120.0 \mathrm{D}$ & $200.0 \mathrm{D}$ & $360.0 \mathrm{D}$ \\
\hline Isotope & $\mathrm{g} / 1 \mathrm{~g}$ of $\mathrm{Nb}$ & $\mathrm{g} / 1 \mathrm{~g}$ of $\mathrm{Nb}$ & $\mathrm{g} / 1 \mathrm{~g}$ of $\mathrm{Nb}$ & $\mathrm{g} / 1 \mathrm{~g}$ of $\mathrm{Nb}$ & $\mathrm{g} / 1 \mathrm{~g}$ of $\mathrm{Nb}$ & $\mathrm{g} / 1 \mathrm{~g}$ of $\mathrm{Nb}$ & $\mathrm{g} / 1 \mathrm{~g}$ of $\mathrm{Nb}$ & $\mathrm{g} / 1 \mathrm{~g}$ of $\mathrm{Nb}$ & $\mathrm{g} / 1 \mathrm{~g}$ of $\mathrm{Nb}$ & $\mathrm{g} / 1 \mathrm{~g}$ of $\mathrm{Nb}$ \\
\hline H 1 & $1.88 \mathrm{E}-08$ & $1.88 \mathrm{E}-08$ & $1.88 \mathrm{E}-08$ & $1.88 \mathrm{E}-08$ & $1.88 \mathrm{E}-08$ & $1.88 \mathrm{E}-08$ & $1.88 \mathrm{E}-08$ & $1.88 \mathrm{E}-08$ & $1.88 \mathrm{E}-08$ & $1.88 \mathrm{E}-08$ \\
\hline H 2 & $6.53 \mathrm{E}-12$ & $6.53 \mathrm{E}-12$ & $6.53 \mathrm{E}-12$ & $6.53 \mathrm{E}-12$ & $6.53 \mathrm{E}-12$ & $6.53 \mathrm{E}-12$ & $6.53 \mathrm{E}-12$ & $6.53 \mathrm{E}-12$ & $6.53 \mathrm{E}-12$ & $6.53 \mathrm{E}-12$ \\
\hline HE 4 & $7.98 \mathrm{E}-09$ & $7.98 \mathrm{E}-09$ & $7.98 \mathrm{E}-09$ & $7.98 \mathrm{E}-09$ & $7.98 \mathrm{E}-09$ & $7.98 \mathrm{E}-09$ & $7.98 \mathrm{E}-09$ & $7.98 \mathrm{E}-09$ & $7.98 \mathrm{E}-09$ & $7.98 \mathrm{E}-09$ \\
\hline SR 90 & $1.29 \mathrm{E}-13$ & $1.29 \mathrm{E}-13$ & $1.29 \mathrm{E}-13$ & $1.29 \mathrm{E}-13$ & $1.28 \mathrm{E}-13$ & $1.28 \mathrm{E}-13$ & $1.28 \mathrm{E}-13$ & $1.28 \mathrm{E}-13$ & $1.27 \mathrm{E}-13$ & $1.26 \mathrm{E}-13$ \\
\hline Y 90 & $3.14 \mathrm{E}-09$ & $3.12 \mathrm{E}-09$ & $2.42 \mathrm{E}-09$ & $5.09 \mathrm{E}-10$ & $1.29 \mathrm{E}-12$ & $5.61 \mathrm{E}-16$ & $3.23 \mathrm{E}-17$ & $3.20 \mathrm{E}-17$ & $3.18 \mathrm{E}-17$ & $3.15 \mathrm{E}-17$ \\
\hline Y 91 & $4.82 \mathrm{E}-12$ & $4.82 \mathrm{E}-12$ & $4.76 \mathrm{E}-12$ & 4.44E-12 & $3.38 \mathrm{E}-12$ & $2.37 \mathrm{E}-12$ & $1.66 \mathrm{E}-12$ & $1.16 \mathrm{E}-12$ & $4.51 \mathrm{E}-13$ & $6.77 \mathrm{E}-14$ \\
\hline ZR 90 & $1.76 \mathrm{E}-07$ & $1.76 \mathrm{E}-07$ & $1.77 \mathrm{E}-07$ & $1.79 \mathrm{E}-07$ & $1.79 \mathrm{E}-07$ & $1.79 \mathrm{E}-07$ & $1.79 \mathrm{E}-07$ & $1.79 \mathrm{E}-07$ & $1.79 \mathrm{E}-07$ & $1.79 \mathrm{E}-07$ \\
\hline ZR 91 & $3.12 \mathrm{E}-11$ & $3.12 \mathrm{E}-11$ & $3.13 \mathrm{E}-11$ & $3.16 \mathrm{E}-11$ & 3.27E-11 & 3.37E-11 & $3.44 \mathrm{E}-11$ & 3.49E-11 & 3.56E-11 & $3.60 \mathrm{E}-11$ \\
\hline ZR 92 & $1.79 \mathrm{E}-10$ & $1.79 \mathrm{E}-10$ & $1.79 \mathrm{E}-10$ & $1.79 \mathrm{E}-10$ & $1.79 \mathrm{E}-10$ & $1.79 \mathrm{E}-10$ & $1.79 \mathrm{E}-10$ & $1.79 \mathrm{E}-10$ & $1.79 \mathrm{E}-10$ & $1.79 \mathrm{E}-10$ \\
\hline ZR 93 & $1.74 \mathrm{E}-06$ & $1.74 \mathrm{E}-06$ & $1.74 \mathrm{E}-06$ & $1.74 \mathrm{E}-06$ & $1.74 \mathrm{E}-06$ & $1.74 \mathrm{E}-06$ & $1.74 \mathrm{E}-06$ & $1.74 \mathrm{E}-06$ & $1.74 \mathrm{E}-06$ & $1.74 \mathrm{E}-06$ \\
\hline ZR 94 & $9.06 \mathrm{E}-09$ & $9.06 \mathrm{E}-09$ & 9.06E-09 & $9.06 \mathrm{E}-09$ & $9.06 \mathrm{E}-09$ & 9.06E-09 & $9.06 \mathrm{E}-09$ & $9.06 \mathrm{E}-09$ & $9.06 \mathrm{E}-09$ & $9.06 \mathrm{E}-09$ \\
\hline ZR 95 & $3.60 \mathrm{E}-13$ & $3.60 \mathrm{E}-13$ & $3.56 \mathrm{E}-13$ & $3.34 \mathrm{E}-13$ & $2.60 \mathrm{E}-13$ & $1.88 \mathrm{E}-13$ & $1.36 \mathrm{E}-13$ & $9.81 \mathrm{E}-14$ & $4.12 \mathrm{E}-14$ & $7.29 \mathrm{E}-15$ \\
\hline NB 93 & $9.95 \mathrm{E}-01$ & $9.95 \mathrm{E}-01$ & $9.95 \mathrm{E}-01$ & $9.95 \mathrm{E}-01$ & $9.95 \mathrm{E}-01$ & $9.95 \mathrm{E}-01$ & $9.95 \mathrm{E}-01$ & $9.95 \mathrm{E}-01$ & $9.95 \mathrm{E}-01$ & $9.95 \mathrm{E}-01$ \\
\hline NB 93M & $2.23 \mathrm{E}-13$ & $2.23 \mathrm{E}-13$ & $2.25 \mathrm{E}-13$ & $2.37 \mathrm{E}-13$ & $2.83 \mathrm{E}-13$ & $3.43 \mathrm{E}-13$ & $4.03 \mathrm{E}-13$ & $4.62 \mathrm{E}-13$ & $6.20 \mathrm{E}-13$ & $9.30 \mathrm{E}-13$ \\
\hline NB 94 & 4.05E-03 & $4.05 \mathrm{E}-03$ & 4.05E-03 & $4.05 \mathrm{E}-03$ & $4.05 \mathrm{E}-03$ & 4.05E-03 & $4.05 \mathrm{E}-03$ & $4.05 \mathrm{E}-03$ & $4.05 \mathrm{E}-03$ & $4.05 \mathrm{E}-03$ \\
\hline NB 95 & $3.14 \mathrm{E}-05$ & $3.14 \mathrm{E}-05$ & $3.08 \mathrm{E}-05$ & $2.74 \mathrm{E}-05$ & $1.74 \mathrm{E}-05$ & $9.63 \mathrm{E}-06$ & $5.33 \mathrm{E}-06$ & $2.95 \mathrm{E}-06$ & $6.09 \mathrm{E}-07$ & $2.60 \mathrm{E}-08$ \\
\hline
\end{tabular}


TEM-10200-1

$12 / 19 / 17$

Rev.08

Title:

AS-RUN NEUTRONICS EVALUATION FOR THE GE HITACHI EXPERIMENT IN THE ATR

ECAR NO.:
ENGINEERING CALCULATIONS AND ANALYSIS

Page $\mathbf{5 5}$ of $\mathbf{5 7}$

\begin{tabular}{|c|c|c|c|c|c|c|c|c|c|c|}
\hline \multicolumn{11}{|c|}{$1 \mathrm{~g}$ of $\mathrm{Nb}$} \\
\hline \multirow{2}{*}{ Isotope } & EOI & $30.0 \mathrm{MI}$ & $1.0 \mathrm{D}$ & $7.0 \mathrm{D}$ & $30.0 \mathrm{D}$ & $60.0 \mathrm{D}$ & $90.0 \mathrm{D}$ & $120.0 \mathrm{D}$ & $200.0 \mathrm{D}$ & $360.0 \mathrm{D}$ \\
\hline & $\mathrm{g} / 1 \mathrm{~g}$ of $\mathrm{Nb}$ & $\mathrm{g} / 1 \mathrm{~g}$ of $\mathrm{Nb}$ & $\mathrm{g} / 1 \mathrm{~g}$ of $\mathrm{Nb}$ & $\mathrm{g} / 1 \mathrm{~g}$ of $\mathrm{Nb}$ & $\mathrm{g} / 1 \mathrm{~g}$ of $\mathrm{Nb}$ & $\mathrm{g} / 1 \mathrm{~g}$ of $\mathrm{Nb}$ & $\mathrm{g} / 1 \mathrm{~g}$ of $\mathrm{Nb}$ & $\mathrm{g} / 1 \mathrm{~g}$ of $\mathrm{Nb}$ & $\mathrm{g} / 1 \mathrm{~g}$ of $\mathrm{Nb}$ & $\mathrm{g} / 1 \mathrm{~g}$ of $\mathrm{Nb}$ \\
\hline NB 96 & $1.72 \mathrm{E}-09$ & $1.69 \mathrm{E}-09$ & $8.43 \mathrm{E}-10$ & $1.17 \mathrm{E}-11$ & $8.97 \mathrm{E}-19$ & $4.68 \mathrm{E}-28$ & $2.45 \mathrm{E}-37$ & $0.00 \mathrm{E}+00$ & $0.00 \mathrm{E}+00$ & $0.00 \mathrm{E}+00$ \\
\hline MO 94 & $4.19 \mathrm{E}-08$ & $4.19 \mathrm{E}-08$ & $4.23 \mathrm{E}-08$ & $4.45 \mathrm{E}-08$ & $5.32 \mathrm{E}-08$ & $6.46 \mathrm{E}-08$ & $7.60 \mathrm{E}-08$ & $8.73 \mathrm{E}-08$ & $1.18 \mathrm{E}-07$ & $1.78 \mathrm{E}-07$ \\
\hline MO 95 & $5.63 \mathrm{E}-05$ & $5.64 \mathrm{E}-05$ & $5.70 \mathrm{E}-05$ & $6.04 \mathrm{E}-05$ & $7.04 \mathrm{E}-05$ & $7.82 \mathrm{E}-05$ & $8.25 \mathrm{E}-05$ & $8.48 \mathrm{E}-05$ & $8.72 \mathrm{E}-05$ & $8.78 \mathrm{E}-05$ \\
\hline MO 96 & $8.08 \mathrm{E}-07$ & $8.08 \mathrm{E}-07$ & $8.09 \mathrm{E}-07$ & $8.10 \mathrm{E}-07$ & $8.10 \mathrm{E}-07$ & $8.10 \mathrm{E}-07$ & $8.10 \mathrm{E}-07$ & $8.10 \mathrm{E}-07$ & $8.10 \mathrm{E}-07$ & $8.10 \mathrm{E}-07$ \\
\hline MO 97 & $1.29 \mathrm{E}-09$ & $1.29 \mathrm{E}-09$ & $1.29 \mathrm{E}-09$ & $1.29 \mathrm{E}-09$ & $1.29 \mathrm{E}-09$ & $1.29 \mathrm{E}-09$ & $1.29 \mathrm{E}-09$ & $1.29 \mathrm{E}-09$ & $1.29 \mathrm{E}-09$ & $1.29 \mathrm{E}-09$ \\
\hline MO 98 & $1.70 \mathrm{E}-12$ & $1.70 \mathrm{E}-12$ & $1.70 \mathrm{E}-12$ & $1.70 \mathrm{E}-12$ & $1.70 \mathrm{E}-12$ & $1.70 \mathrm{E}-12$ & $1.70 \mathrm{E}-12$ & $1.70 \mathrm{E}-12$ & $1.70 \mathrm{E}-12$ & $1.70 \mathrm{E}-12$ \\
\hline SUMTOT & $9.99 \mathrm{E}-01$ & $9.99 \mathrm{E}-01$ & 9.99E-01 & $9.99 \mathrm{E}-01$ & $9.99 \mathrm{E}-01$ & 9.99E-01 & $9.99 \mathrm{E}-01$ & $9.99 \mathrm{E}-01$ & $9.99 \mathrm{E}-01$ & $9.99 \mathrm{E}-01$ \\
\hline OTOTAL & $9.99 \mathrm{E}-01$ & $9.99 \mathrm{E}-01$ & 9.99E-01 & $9.99 \mathrm{E}-01$ & $9.99 \mathrm{E}-01$ & 9.99E-01 & $9.99 \mathrm{E}-01$ & $9.99 \mathrm{E}-01$ & $9.99 \mathrm{E}-01$ & $9.99 \mathrm{E}-01$ \\
\hline
\end{tabular}

Table 24. $\mathrm{Ci} / 1 \mathrm{~g}$ of $\mathrm{Nb}$

\begin{tabular}{|c|c|c|c|c|c|c|c|c|c|c|}
\hline \multicolumn{11}{|c|}{$1 \mathrm{~g}$ of $\mathrm{Nb}$} \\
\hline \multirow{2}{*}{ Isotope } & EOI & $30.0 \mathrm{MI}$ & $1.0 \mathrm{D}$ & $7.0 \mathrm{D}$ & $30.0 \mathrm{D}$ & $60.0 \mathrm{D}$ & $90.0 \mathrm{D}$ & $120.0 \mathrm{D}$ & $200.0 \mathrm{D}$ & $360.0 \mathrm{D}$ \\
\hline & $\mathrm{Ci} / 1 \mathrm{~g}$ of $\mathrm{Nb}$ & $\mathrm{Ci} / 1 \mathrm{~g}$ of $\mathrm{Nb}$ & $\mathrm{Ci} / 1 \mathrm{~g}$ of $\mathrm{Nb}$ & $\mathrm{Ci} / 1 \mathrm{~g}$ of $\mathrm{Nb}$ & $\mathrm{Ci} / 1 \mathrm{~g}$ of $\mathrm{Nb}$ & $\mathrm{Ci} / 1 \mathrm{~g}$ of $\mathrm{Nb}$ & $\mathrm{Ci} / 1 \mathrm{~g}$ of $\mathrm{Nb}$ & $\mathrm{Ci} / 1 \mathrm{~g}$ of $\mathrm{Nb}$ & $\mathrm{Ci} / 1 \mathrm{~g}$ of $\mathrm{Nb}$ & $\mathrm{Ci} / 1 \mathrm{~g}$ of $\mathrm{Nb}$ \\
\hline H 3 & $1.53 \mathrm{E}-14$ & $1.53 \mathrm{E}-14$ & $1.53 \mathrm{E}-14$ & $1.53 \mathrm{E}-14$ & $1.53 \mathrm{E}-14$ & $1.52 \mathrm{E}-14$ & $1.51 \mathrm{E}-14$ & $1.51 \mathrm{E}-14$ & $1.49 \mathrm{E}-14$ & $1.45 \mathrm{E}-14$ \\
\hline SR 89 & $1.60 \mathrm{E}-13$ & $1.60 \mathrm{E}-13$ & $1.58 \mathrm{E}-13$ & $1.46 \mathrm{E}-13$ & $1.06 \mathrm{E}-13$ & 7.03E-14 & $4.66 \mathrm{E}-14$ & $3.09 \mathrm{E}-14$ & $1.03 \mathrm{E}-14$ & $1.15 \mathrm{E}-15$ \\
\hline SR 90 & $1.75 \mathrm{E}-11$ & $1.75 \mathrm{E}-11$ & $1.75 \mathrm{E}-11$ & $1.75 \mathrm{E}-11$ & $1.75 \mathrm{E}-11$ & $1.75 \mathrm{E}-11$ & $1.74 \mathrm{E}-11$ & $1.74 \mathrm{E}-11$ & $1.73 \mathrm{E}-11$ & $1.71 \mathrm{E}-11$ \\
\hline SR 91 & 4.63E-12 & 4.47E-12 & $8.04 \mathrm{E}-13$ & $2.20 \mathrm{E}-17$ & $0.00 \mathrm{E}+00$ & $0.00 \mathrm{E}+00$ & $0.00 \mathrm{E}+00$ & $0.00 \mathrm{E}+00$ & $0.00 \mathrm{E}+00$ & $0.00 \mathrm{E}+00$ \\
\hline Y 90 & $1.71 \mathrm{E}-03$ & $1.70 \mathrm{E}-03$ & $1.32 \mathrm{E}-03$ & $2.77 \mathrm{E}-04$ & 7.01E-07 & $3.05 \mathrm{E}-10$ & $1.76 \mathrm{E}-11$ & $1.74 \mathrm{E}-11$ & $1.73 \mathrm{E}-11$ & $1.71 \mathrm{E}-11$ \\
\hline Y 91 & $1.18 \mathrm{E}-07$ & $1.18 \mathrm{E}-07$ & $1.17 \mathrm{E}-07$ & $1.09 \mathrm{E}-07$ & $8.29 \mathrm{E}-08$ & $5.81 \mathrm{E}-08$ & 4.07E-08 & $2.85 \mathrm{E}-08$ & $1.11 \mathrm{E}-08$ & $1.66 \mathrm{E}-09$ \\
\hline Y 92 & 7.62E-11 & $6.91 \mathrm{E}-11$ & $6.93 \mathrm{E}-13$ & $3.90 \mathrm{E}-25$ & $0.00 \mathrm{E}+00$ & $0.00 \mathrm{E}+00$ & $0.00 E+00$ & $0.00 \mathrm{E}+00$ & $0.00 \mathrm{E}+00$ & $0.00 \mathrm{E}+00$ \\
\hline Y 94 & $2.55 \mathrm{E}-12$ & $8.58 \mathrm{E}-13$ & $0.00 \mathrm{E}+00$ & $0.00 \mathrm{E}+00$ & $0.00 \mathrm{E}+00$ & $0.00 \mathrm{E}+00$ & $0.00 E+00$ & $0.00 \mathrm{E}+00$ & $0.00 \mathrm{E}+00$ & $0.00 \mathrm{E}+00$ \\
\hline ZR 93 & $4.36 \mathrm{E}-09$ & $4.36 \mathrm{E}-09$ & $4.36 \mathrm{E}-09$ & $4.36 \mathrm{E}-09$ & $4.36 \mathrm{E}-09$ & $4.36 \mathrm{E}-09$ & $4.36 \mathrm{E}-09$ & $4.36 \mathrm{E}-09$ & $4.36 \mathrm{E}-09$ & $4.36 \mathrm{E}-09$ \\
\hline ZR 95 & $7.74 \mathrm{E}-09$ & 7.73E-09 & $7.65 \mathrm{E}-09$ & $7.17 \mathrm{E}-09$ & $5.59 \mathrm{E}-09$ & $4.04 \mathrm{E}-09$ & $2.92 \mathrm{E}-09$ & $2.11 \mathrm{E}-09$ & $8.86 \mathrm{E}-10$ & $1.57 \mathrm{E}-10$ \\
\hline ZR 97 & $3.62 \mathrm{E}-15$ & 3.54E-15 & $1.35 \mathrm{E}-15$ & $0.00 \mathrm{E}+00$ & $0.00 \mathrm{E}+00$ & $0.00 \mathrm{E}+00$ & $0.00 \mathrm{E}+00$ & $0.00 \mathrm{E}+00$ & $0.00 \mathrm{E}+00$ & $0.00 \mathrm{E}+00$ \\
\hline NB 93M & $6.30 \mathrm{E}-11$ & $6.30 \mathrm{E}-11$ & $6.35 \mathrm{E}-11$ & $6.69 \mathrm{E}-11$ & $8.00 \mathrm{E}-11$ & $9.70 \mathrm{E}-11$ & $1.14 \mathrm{E}-10$ & $1.31 \mathrm{E}-10$ & $1.75 \mathrm{E}-10$ & $2.63 \mathrm{E}-10$ \\
\hline NB 94 & $7.59 \mathrm{E}-04$ & $7.59 \mathrm{E}-04$ & $7.59 \mathrm{E}-04$ & $7.59 \mathrm{E}-04$ & $7.59 \mathrm{E}-04$ & $7.59 \mathrm{E}-04$ & $7.59 \mathrm{E}-04$ & $7.59 \mathrm{E}-04$ & $7.59 \mathrm{E}-04$ & $7.59 \mathrm{E}-04$ \\
\hline NB 95 & $1.23 \mathrm{E}+00$ & $1.23 \mathrm{E}+00$ & $1.21 \mathrm{E}+00$ & $1.07 \mathrm{E}+00$ & $6.81 \mathrm{E}-01$ & $3.77 \mathrm{E}-01$ & $2.09 \mathrm{E}-01$ & $1.15 \mathrm{E}-01$ & $2.38 \mathrm{E}-02$ & $1.02 \mathrm{E}-03$ \\
\hline NB 95M & $5.10 \mathrm{E}-11$ & $5.10 \mathrm{E}-11$ & $5.15 \mathrm{E}-11$ & $5.15 \mathrm{E}-11$ & $4.14 \mathrm{E}-11$ & $3.00 \mathrm{E}-11$ & $2.16 \mathrm{E}-11$ & $1.56 \mathrm{E}-11$ & $6.57 \mathrm{E}-12$ & $1.16 \mathrm{E}-12$ \\
\hline NB 96 & $2.40 \mathrm{E}-03$ & $2.37 \mathrm{E}-03$ & $1.18 \mathrm{E}-03$ & $1.64 \mathrm{E}-05$ & $1.26 \mathrm{E}-12$ & $6.55 \mathrm{E}-22$ & $3.42 \mathrm{E}-31$ & $0.00 \mathrm{E}+00$ & $0.00 \mathrm{E}+00$ & $0.00 \mathrm{E}+00$ \\
\hline NB 97 & $4.58 \mathrm{E}-12$ & $3.44 \mathrm{E}-12$ & $1.36 \mathrm{E}-15$ & $3.69 \mathrm{E}-18$ & $0.00 \mathrm{E}+00$ & $0.00 \mathrm{E}+00$ & $0.00 \mathrm{E}+00$ & $0.00 \mathrm{E}+00$ & $0.00 \mathrm{E}+00$ & $0.00 \mathrm{E}+00$ \\
\hline NB 97M & $3.42 \mathrm{E}-15$ & 3.36E-15 & $1.28 \mathrm{E}-15$ & $3.47 \mathrm{E}-18$ & $0.00 \mathrm{E}+00$ & $0.00 \mathrm{E}+00$ & $0.00 \mathrm{E}+00$ & $0.00 \mathrm{E}+00$ & $0.00 \mathrm{E}+00$ & $0.00 \mathrm{E}+00$ \\
\hline MO 99 & $3.21 \mathrm{E}-11$ & $3.20 \mathrm{E}-11$ & $2.50 \mathrm{E}-11$ & $5.50 \mathrm{E}-12$ & $1.67 \mathrm{E}-14$ & $8.69 \mathrm{E}-18$ & $4.52 \mathrm{E}-21$ & $2.35 \mathrm{E}-24$ & $0.00 \mathrm{E}+00$ & $0.00 \mathrm{E}+00$ \\
\hline TC100 & $5.59 \mathrm{E}-14$ & $0.00 \mathrm{E}+00$ & $0.00 \mathrm{E}+00$ & $0.00 \mathrm{E}+00$ & $0.00 \mathrm{E}+00$ & $0.00 \mathrm{E}+00$ & $0.00 \mathrm{E}+00$ & $0.00 \mathrm{E}+00$ & $0.00 \mathrm{E}+00$ & $0.00 \mathrm{E}+00$ \\
\hline SUMTOT & $1.24 \mathrm{E}+00$ & $1.23 \mathrm{E}+00$ & $1.21 \mathrm{E}+00$ & $1.07 \mathrm{E}+00$ & $6.81 \mathrm{E}-01$ & $3.78 \mathrm{E}-01$ & $2.09 \mathrm{E}-01$ & $1.16 \mathrm{E}-01$ & $2.46 \mathrm{E}-02$ & $1.78 \mathrm{E}-03$ \\
\hline
\end{tabular}


TEM-10200-1

$12 / 19 / 17$

Rev.08

Title:

AS-RUN NEUTRONICS EVALUATION FOR THE GE HITACHI EXPERIMENT IN THE ATR

ECAR NO.: 4740
PROJECT NO.: 32535 Date: 12/02/2019

\begin{tabular}{|c|c|c|c|c|c|c|c|c|c|c|}
\hline \multicolumn{11}{|c|}{$1 \mathrm{~g}$ of $\mathrm{Nb}$} \\
\hline Iscton & EOI & $30.0 \mathrm{MI}$ & $1.0 \mathrm{D}$ & $7.0 \mathrm{D}$ & $30.0 \mathrm{D}$ & $60.0 \mathrm{D}$ & $90.0 \mathrm{D}$ & $120.0 \mathrm{D}$ & $200.0 \mathrm{D}$ & $360.0 \mathrm{D}$ \\
\hline Isotope & $\mathrm{Ci} / 1 \mathrm{~g}$ of $\mathrm{Nb}$ & $\mathrm{Ci} / 1 \mathrm{~g}$ of $\mathrm{Nb}$ & $\mathrm{Ci} / 1 \mathrm{~g}$ of $\mathrm{Nb}$ & $\mathrm{Ci} / 1 \mathrm{~g}$ of $\mathrm{Nb}$ & $\mathrm{Ci} / 1 \mathrm{~g}$ of $\mathrm{Nb}$ & $\mathrm{Ci} / 1 \mathrm{~g}$ of $\mathrm{Nb}$ & $\mathrm{Ci} / 1 \mathrm{~g}$ of $\mathrm{Nb}$ & $\mathrm{Ci} / 1 \mathrm{~g}$ of $\mathrm{Nb}$ & $\mathrm{Ci} / 1 \mathrm{~g}$ of $\mathrm{Nb}$ & $\mathrm{Ci} / 1 \mathrm{~g}$ of $\mathrm{Nb}$ \\
\hline OTOTAL & $1.24 \mathrm{E}+00$ & $1.23 \mathrm{E}+00$ & $1.21 \mathrm{E}+00$ & $1.07 \mathrm{E}+00$ & $6.81 \mathrm{E}-01$ & $3.78 \mathrm{E}-01$ & $2.09 \mathrm{E}-01$ & 1.16E-01 & $2.46 \mathrm{E}-02$ & $1.78 \mathrm{E}-03$ \\
\hline
\end{tabular}

Table 25. g/1g of Fe

\begin{tabular}{|c|c|c|c|c|c|c|c|c|c|c|}
\hline \multicolumn{11}{|c|}{$1 \mathrm{~g}$ of Fe } \\
\hline \multirow{2}{*}{ Isotope } & EOI & $30.0 \mathrm{MI}$ & $1.0 \mathrm{D}$ & $7.0 \mathrm{D}$ & $30.0 \mathrm{D}$ & $60.0 \mathrm{D}$ & $90.0 \mathrm{D}$ & $120.0 \mathrm{D}$ & $200.0 \mathrm{D}$ & $360.0 \mathrm{D}$ \\
\hline & $\mathrm{g} / 1 \mathrm{~g}$ of $\mathrm{Fe}$ & $\mathrm{g} / 1 \mathrm{~g}$ of $\mathrm{Fe}$ & $\mathrm{g} / 1 \mathrm{~g}$ of $\mathrm{Fe}$ & $\mathrm{g} / 1 \mathrm{~g}$ of $\mathrm{Fe}$ & $\mathrm{g} / 1 \mathrm{~g}$ of $\mathrm{Fe}$ & $\mathrm{g} / 1 \mathrm{~g}$ of $\mathrm{Fe}$ & $\mathrm{g} / 1 \mathrm{~g}$ of $\mathrm{Fe}$ & $\mathrm{g} / 1 \mathrm{~g}$ of $\mathrm{Fe}$ & $\mathrm{g} / 1 \mathrm{~g}$ of $\mathrm{Fe}$ & $\mathrm{g} / 1 \mathrm{~g}$ of $\mathrm{Fe}$ \\
\hline H 1 & $4.85 \mathrm{E}-07$ & $4.85 \mathrm{E}-07$ & $4.85 \mathrm{E}-07$ & $4.85 \mathrm{E}-07$ & $4.85 \mathrm{E}-07$ & $4.85 \mathrm{E}-07$ & $4.85 \mathrm{E}-07$ & $4.85 \mathrm{E}-07$ & $4.85 \mathrm{E}-07$ & $4.85 \mathrm{E}-07$ \\
\hline H 2 & $1.69 \mathrm{E}-10$ & $1.69 \mathrm{E}-10$ & $1.69 \mathrm{E}-10$ & $1.69 \mathrm{E}-10$ & $1.69 \mathrm{E}-10$ & $1.69 \mathrm{E}-10$ & $1.69 \mathrm{E}-10$ & $1.69 \mathrm{E}-10$ & $1.69 \mathrm{E}-10$ & $1.69 \mathrm{E}-10$ \\
\hline HE 4 & 1.06E-07 & $1.06 \mathrm{E}-07$ & 1.06E-07 & 1.06E-07 & 1.06E-07 & 1.06E-07 & 1.06E-07 & $1.06 \mathrm{E}-07$ & $1.06 \mathrm{E}-07$ & 1.06E-07 \\
\hline TI 48 & $1.37 \mathrm{E}-15$ & $1.37 \mathrm{E}-15$ & $1.39 \mathrm{E}-15$ & $1.41 \mathrm{E}-15$ & $1.41 \mathrm{E}-15$ & $1.41 \mathrm{E}-15$ & $1.41 \mathrm{E}-15$ & $1.41 \mathrm{E}-15$ & $1.41 \mathrm{E}-15$ & $1.41 \mathrm{E}-15$ \\
\hline TI 50 & $3.32 \mathrm{E}-12$ & $3.32 \mathrm{E}-12$ & $3.32 \mathrm{E}-12$ & $3.32 \mathrm{E}-12$ & $3.32 \mathrm{E}-12$ & $3.32 \mathrm{E}-12$ & $3.32 \mathrm{E}-12$ & $3.32 \mathrm{E}-12$ & $3.32 \mathrm{E}-12$ & $3.32 \mathrm{E}-12$ \\
\hline V 51 & $3.18 \mathrm{E}-07$ & $3.18 \mathrm{E}-07$ & $3.20 \mathrm{E}-07$ & $3.30 \mathrm{E}-07$ & $3.56 \mathrm{E}-07$ & $3.73 \mathrm{E}-07$ & $3.81 \mathrm{E}-07$ & $3.85 \mathrm{E}-07$ & $3.88 \mathrm{E}-07$ & 3.89E-07 \\
\hline$V 52$ & $2.57 \mathrm{E}-14$ & $1.00 \mathrm{E}-16$ & $0.00 \mathrm{E}+00$ & $0.00 \mathrm{E}+00$ & $0.00 \mathrm{E}+00$ & $0.00 \mathrm{E}+00$ & $0.00 \mathrm{E}+00$ & $0.00 \mathrm{E}+00$ & $0.00 \mathrm{E}+00$ & $0.00 \mathrm{E}+00$ \\
\hline CR 51 & 7.06E-08 & $7.06 \mathrm{E}-08$ & $6.89 \mathrm{E}-08$ & $5.93 \mathrm{E}-08$ & $3.33 \mathrm{E}-08$ & 1.57E-08 & $7.43 \mathrm{E}-09$ & $3.51 \mathrm{E}-09$ & $4.74 \mathrm{E}-10$ & $8.67 \mathrm{E}-12$ \\
\hline CR 52 & $6.87 \mathrm{E}-10$ & $6.87 \mathrm{E}-10$ & $6.87 \mathrm{E}-10$ & $6.87 \mathrm{E}-10$ & $6.87 \mathrm{E}-10$ & $6.87 \mathrm{E}-10$ & $6.87 \mathrm{E}-10$ & $6.87 \mathrm{E}-10$ & $6.87 \mathrm{E}-10$ & 6.87E-10 \\
\hline CR 53 & $7.62 \mathrm{E}-07$ & $7.62 \mathrm{E}-07$ & $7.62 \mathrm{E}-07$ & $7.62 \mathrm{E}-07$ & 7.62E-07 & 7.62E- 07 & $7.62 \mathrm{E}-07$ & $7.62 \mathrm{E}-07$ & $7.62 \mathrm{E}-07$ & $7.62 \mathrm{E}-07$ \\
\hline CR 54 & $2.60 \mathrm{E}-06$ & $2.61 \mathrm{E}-06$ & $2.62 \mathrm{E}-06$ & $2.74 \mathrm{E}-06$ & $3.18 \mathrm{E}-06$ & $3.72 \mathrm{E}-06$ & 4.23E-06 & $4.70 \mathrm{E}-06$ & $5.82 \mathrm{E}-06$ & $7.54 \mathrm{E}-06$ \\
\hline CR 55 & 1.65E-14 & $4.69 \mathrm{E}-17$ & $0.00 \mathrm{E}+00$ & $0.00 \mathrm{E}+00$ & $0.00 \mathrm{E}+00$ & $0.00 \mathrm{E}+00$ & $0.00 \mathrm{E}+00$ & $0.00 \mathrm{E}+00$ & $0.00 \mathrm{E}+00$ & $0.00 \mathrm{E}+00$ \\
\hline MN 54 & $8.97 \mathrm{E}-06$ & $8.97 \mathrm{E}-06$ & $8.95 \mathrm{E}-06$ & $8.83 \mathrm{E}-06$ & $8.39 \mathrm{E}-06$ & $7.85 \mathrm{E}-06$ & $7.34 \mathrm{E}-06$ & $6.87 \mathrm{E}-06$ & $5.75 \mathrm{E}-06$ & $4.04 \mathrm{E}-06$ \\
\hline MN 55 & $9.19 \mathrm{E}-06$ & 9.19E-06 & $9.27 \mathrm{E}-06$ & 9.76E-06 & 1.16E-05 & 1.40E-05 & 1.63E-05 & $1.86 \mathrm{E}-05$ & $2.44 \mathrm{E}-05$ & 3.50E-05 \\
\hline MN 56 & 1.09E-08 & $9.56 \mathrm{E}-09$ & $1.72 \mathrm{E}-11$ & $2.67 \mathrm{E}-28$ & $0.00 \mathrm{E}+00$ & $0.00 \mathrm{E}+00$ & $0.00 \mathrm{E}+00$ & $0.00 \mathrm{E}+00$ & $0.00 \mathrm{E}+00$ & $0.00 \mathrm{E}+00$ \\
\hline MN 57 & $2.21 \mathrm{E}-13$ & 5.43E-19 & $0.00 \mathrm{E}+00$ & $0.00 \mathrm{E}+00$ & $0.00 E+00$ & $0.00 \mathrm{E}+00$ & $0.00 \mathrm{E}+00$ & $0.00 \mathrm{E}+00$ & $0.00 \mathrm{E}+00$ & $0.00 \mathrm{E}+00$ \\
\hline FE 54 & $5.60 \mathrm{E}-02$ & $5.60 \mathrm{E}-02$ & $5.60 \mathrm{E}-02$ & $5.60 \mathrm{E}-02$ & $5.60 \mathrm{E}-02$ & $5.60 \mathrm{E}-02$ & $5.60 \mathrm{E}-02$ & $5.60 \mathrm{E}-02$ & $5.60 \mathrm{E}-02$ & 5.60E-02 \\
\hline FE 55 & $1.12 \mathrm{E}-04$ & $1.12 \mathrm{E}-04$ & 1.12E-04 & $1.11 \mathrm{E}-04$ & 1.09E-04 & 1.07E-04 & $1.05 \mathrm{E}-04$ & $1.02 \mathrm{E}-04$ & $9.66 \mathrm{E}-05$ & $8.59 \mathrm{E}-05$ \\
\hline FE 56 & 9.17E-01 & $9.17 \mathrm{E}-01$ & 9.17E-01 & 9.17E-01 & 9.17E-01 & 9.17E-01 & 9.17E-01 & $9.17 \mathrm{E}-01$ & $9.17 \mathrm{E}-01$ & 9.17E-01 \\
\hline FE 57 & $2.44 \mathrm{E}-02$ & $2.44 \mathrm{E}-02$ & $2.44 \mathrm{E}-02$ & $2.44 \mathrm{E}-02$ & $2.44 \mathrm{E}-02$ & $2.44 \mathrm{E}-02$ & $2.44 \mathrm{E}-02$ & $2.44 \mathrm{E}-02$ & $2.44 \mathrm{E}-02$ & $2.44 \mathrm{E}-02$ \\
\hline FE 58 & $3.06 \mathrm{E}-03$ & $3.06 \mathrm{E}-03$ & $3.06 \mathrm{E}-03$ & $3.06 \mathrm{E}-03$ & $3.06 \mathrm{E}-03$ & $3.06 \mathrm{E}-03$ & $3.06 \mathrm{E}-03$ & $3.06 \mathrm{E}-03$ & $3.06 \mathrm{E}-03$ & $3.06 \mathrm{E}-03$ \\
\hline FE 59 & 1.09E-06 & $1.08 \mathrm{E}-06$ & 1.07E-06 & $9.74 \mathrm{E}-07$ & $\begin{array}{l}6.83 \mathrm{E}-07 \\
\end{array}$ & $\begin{array}{l}4.31 \mathrm{E}-07 \\
\end{array}$ & $2.71 \mathrm{E}-07$ & $1.71 \mathrm{E}-07$ & $4.98 \mathrm{E}-08$ & $4.24 \mathrm{E}-09$ \\
\hline $\mathrm{CO} 59$ & $2.64 \mathrm{E}-06$ & $2.64 \mathrm{E}-06$ & $2.66 \mathrm{E}-06$ & $2.75 \mathrm{E}-06$ & $3.04 \mathrm{E}-06$ & $3.30 \mathrm{E}-06$ & $3.46 \mathrm{E}-06$ & $3.56 \mathrm{E}-06$ & $3.68 \mathrm{E}-06$ & $3.72 \mathrm{E}-06$ \\
\hline $\mathrm{CO} 60$ & 5.17E-08 & $5.17 \mathrm{E}-08$ & 5.17E-08 & $5.16 \mathrm{E}-08$ & $5.12 \mathrm{E}-08$ & 5.06E-08 & $5.01 \mathrm{E}-08$ & $4.95 \mathrm{E}-08$ & $4.81 \mathrm{E}-08$ & $4.54 \mathrm{E}-08$ \\
\hline CO 60M & $3.37 \mathrm{E}-12$ & $4.63 \mathrm{E}-13$ & $0.00 \mathrm{E}+00$ & $0.00 \mathrm{E}+00$ & $0.00 \mathrm{E}+00$ & $0.00 \mathrm{E}+00$ & $0.00 \mathrm{E}+00$ & $0.00 \mathrm{E}+00$ & $0.00 \mathrm{E}+00$ & $0.00 \mathrm{E}+00$ \\
\hline $\mathrm{CO} 61$ & $6.39 \mathrm{E}-14$ & $5.18 \mathrm{E}-14$ & $2.67 \mathrm{E}-18$ & $0.00 \mathrm{E}+00$ & $0.00 \mathrm{E}+00$ & $0.00 \mathrm{E}+00$ & $0.00 \mathrm{E}+00$ & $0.00 \mathrm{E}+00$ & $0.00 \mathrm{E}+00$ & $0.00 \mathrm{E}+00$ \\
\hline $\mathrm{NI} 60$ & $1.23 \mathrm{E}-09$ & $1.23 \mathrm{E}-09$ & $1.25 \mathrm{E}-09$ & $1.36 \mathrm{E}-09$ & $1.79 \mathrm{E}-09$ & $2.34 \mathrm{E}-09$ & $2.88 \mathrm{E}-09$ & $3.42 \mathrm{E}-09$ & $4.82 \mathrm{E}-09$ & $7.52 \mathrm{E}-09$ \\
\hline NI 61 & $4.08 \mathrm{E}-11$ & $4.08 \mathrm{E}-11$ & $4.08 \mathrm{E}-11$ & $4.08 \mathrm{E}-11$ & $4.08 \mathrm{E}-11$ & $4.08 \mathrm{E}-11$ & $4.08 \mathrm{E}-11$ & $4.08 \mathrm{E}-11$ & $4.08 \mathrm{E}-11$ & $4.08 \mathrm{E}-11$ \\
\hline
\end{tabular}


TEM-10200-1

$12 / 19 / 17$

Rev.08

Title:

AS-RUN NEUTRONICS EVALUATION FOR THE GE HITACHI EXPERIMENT IN THE ATR

ECAR NO.: $\quad 4740$

\section{ENGINEERING CALCULATIONS AND ANALYSIS}

PROJECT NO.: 32535 Date: 12/02/2019

\begin{tabular}{|c|c|c|c|c|c|c|c|c|c|c|}
\hline \multicolumn{11}{|c|}{$1 \mathrm{~g}$ of Fe } \\
\hline \multirow{2}{*}{ Isotope } & EOI & $30.0 \mathrm{MI}$ & $1.0 \mathrm{D}$ & $7.0 \mathrm{D}$ & $30.0 \mathrm{D}$ & $60.0 \mathrm{D}$ & $90.0 \mathrm{D}$ & $120.0 \mathrm{D}$ & $200.0 \mathrm{D}$ & $360.0 \mathrm{D}$ \\
\hline & $\mathrm{g} / 1 \mathrm{~g}$ of $\mathrm{Fe}$ & $\mathrm{g} / 1 \mathrm{~g}$ of $\mathrm{Fe}$ & $\mathrm{g} / 1 \mathrm{~g}$ of $\mathrm{Fe}$ & $\mathrm{g} / 1 \mathrm{~g}$ of $\mathrm{Fe}$ & $\mathrm{g} / 1 \mathrm{~g}$ of Fe & $\mathrm{g} / 1 \mathrm{~g}$ of $\mathrm{Fe}$ & $\mathrm{g} / 1 \mathrm{~g}$ of $\mathrm{Fe}$ & $\mathrm{g} / 1 \mathrm{~g}$ of $\mathrm{Fe}$ & $\mathrm{g} / 1 \mathrm{~g}$ of $\mathrm{Fe}$ & $\mathrm{g} / 1 \mathrm{~g}$ of $\mathrm{Fe}$ \\
\hline NI 62 & $2.18 \mathrm{E}-14$ & $2.18 \mathrm{E}-14$ & $2.18 \mathrm{E}-14$ & $2.18 \mathrm{E}-14$ & $2.18 \mathrm{E}-14$ & $2.18 \mathrm{E}-14$ & $2.18 \mathrm{E}-14$ & $2.18 \mathrm{E}-14$ & $2.18 \mathrm{E}-14$ & $2.18 \mathrm{E}-14$ \\
\hline SUMTOT & $1.00 \mathrm{E}+00$ & $1.00 \mathrm{E}+00$ & $1.00 \mathrm{E}+00$ & $1.00 \mathrm{E}+00$ & $1.00 \mathrm{E}+00$ & $1.00 \mathrm{E}+00$ & $1.00 \mathrm{E}+00$ & $1.00 \mathrm{E}+00$ & $1.00 \mathrm{E}+00$ & $1.00 \mathrm{E}+00$ \\
\hline OTOTAL & $1.00 \mathrm{E}+00$ & $1.00 \mathrm{E}+00$ & $1.00 \mathrm{E}+00$ & $1.00 \mathrm{E}+00$ & $1.00 \mathrm{E}+00$ & $1.00 \mathrm{E}+00$ & $1.00 \mathrm{E}+00$ & $1.00 \mathrm{E}+00$ & $1.00 \mathrm{E}+00$ & $1.00 \mathrm{E}+00$ \\
\hline
\end{tabular}

Table 26. $\mathrm{Ci} / 1 \mathrm{~g}$ of Fe

\begin{tabular}{|c|c|c|c|c|c|c|c|c|c|c|}
\hline \multicolumn{11}{|c|}{$1 \mathrm{~g}$ of Fe } \\
\hline \multirow{2}{*}{ Isotope } & $\mathrm{EOI}$ & $30.0 \mathrm{MI}$ & $1.0 \mathrm{D}$ & $7.0 \mathrm{D}$ & $30.0 \mathrm{D}$ & $60.0 \mathrm{D}$ & $90.0 \mathrm{D}$ & $120.0 \mathrm{D}$ & $200.0 \mathrm{D}$ & $360.0 \mathrm{D}$ \\
\hline & $\mathrm{Ci} / 1 \mathrm{~g}$ of Fe & $\mathrm{Ci} / 1 \mathrm{~g}$ of $\mathrm{Fe}$ & $\mathrm{Ci} / 1 \mathrm{~g}$ of $\mathrm{Fe}$ & $\mathrm{Ci} / 1 \mathrm{~g}$ of $\mathrm{Fe}$ & $\mathrm{Ci} / 1 \mathrm{~g}$ of $\mathrm{Fe}$ & $\mathrm{Ci} / 1 \mathrm{~g}$ of $\mathrm{Fe}$ & $\mathrm{Ci} / 1 \mathrm{~g}$ of $\mathrm{Fe}$ & $\mathrm{Ci} / 1 \mathrm{~g}$ of $\mathrm{Fe}$ & $\mathrm{Ci} / 1 \mathrm{~g}$ of $\mathrm{Fe}$ & $\mathrm{Ci} / 1 \mathrm{~g}$ of $\mathrm{Fe}$ \\
\hline H 3 & $3.96 \mathrm{E}-13$ & $3.96 \mathrm{E}-13$ & $3.96 \mathrm{E}-13$ & $3.96 \mathrm{E}-13$ & $3.95 \mathrm{E}-13$ & $3.93 \mathrm{E}-13$ & $3.91 \mathrm{E}-13$ & $3.89 \mathrm{E}-13$ & $3.84 \mathrm{E}-13$ & $3.75 \mathrm{E}-13$ \\
\hline SC 48 & $5.74 \mathrm{E}-11$ & $5.70 \mathrm{E}-11$ & $3.93 \mathrm{E}-11$ & $4.03 \mathrm{E}-12$ & $6.48 \mathrm{E}-16$ & $7.30 \mathrm{E}-21$ & $8.24 \mathrm{E}-26$ & $9.36 \mathrm{E}-31$ & $0.00 \mathrm{E}+00$ & $0.00 \mathrm{E}+00$ \\
\hline $\mathrm{SC} 50$ & $8.76 \mathrm{E}-15$ & $4.53 \mathrm{E}-20$ & $0.00 \mathrm{E}+00$ & $0.00 \mathrm{E}+00$ & $0.00 \mathrm{E}+00$ & $0.00 \mathrm{E}+00$ & $0.00 \mathrm{E}+00$ & $0.00 \mathrm{E}+00$ & $0.00 \mathrm{E}+00$ & $0.00 \mathrm{E}+00$ \\
\hline TI 51 & 7.51E-09 & $2.03 \mathrm{E}-10$ & $0.00 \mathrm{E}+00$ & $0.00 \mathrm{E}+00$ & $0.00 \mathrm{E}+00$ & $0.00 \mathrm{E}+00$ & $0.00 \mathrm{E}+00$ & $0.00 \mathrm{E}+00$ & $0.00 \mathrm{E}+00$ & $0.00 \mathrm{E}+00$ \\
\hline V 52 & $2.48 \mathrm{E}-05$ & $9.69 \mathrm{E}-08$ & $0.00 \mathrm{E}+00$ & $0.00 \mathrm{E}+00$ & $0.00 \mathrm{E}+00$ & $0.00 \mathrm{E}+00$ & $0.00 \mathrm{E}+00$ & $0.00 \mathrm{E}+00$ & $0.00 \mathrm{E}+00$ & $0.00 \mathrm{E}+00$ \\
\hline V 53 & $3.76 \mathrm{E}-09$ & $9.25 \mathrm{E}-15$ & $0.00 \mathrm{E}+00$ & $0.00 \mathrm{E}+00$ & $0.00 \mathrm{E}+00$ & $0.00 \mathrm{E}+00$ & $0.00 \mathrm{E}+00$ & $0.00 \mathrm{E}+00$ & $0.00 \mathrm{E}+00$ & $0.00 \mathrm{E}+00$ \\
\hline V 54 & $5.09 \mathrm{E}-10$ & $7.16 \mathrm{E}-20$ & $0.00 \mathrm{E}+00$ & $0.00 \mathrm{E}+00$ & $0.00 \mathrm{E}+00$ & $0.00 \mathrm{E}+00$ & $0.00 \mathrm{E}+00$ & $0.00 \mathrm{E}+00$ & $0.00 \mathrm{E}+00$ & $0.00 \mathrm{E}+00$ \\
\hline CR 51 & $6.53 \mathrm{E}-03$ & $6.53 \mathrm{E}-03$ & $6.37 \mathrm{E}-03$ & $5.48 \mathrm{E}-03$ & $3.08 \mathrm{E}-03$ & $1.46 \mathrm{E}-03$ & $6.87 \mathrm{E}-04$ & $3.24 \mathrm{E}-04$ & $4.39 \mathrm{E}-05$ & $8.01 \mathrm{E}-07$ \\
\hline CR 55 & $1.58 \mathrm{E}-05$ & $4.52 \mathrm{E}-08$ & $0.00 \mathrm{E}+00$ & $0.00 \mathrm{E}+00$ & $0.00 \mathrm{E}+00$ & $0.00 \mathrm{E}+00$ & $0.00 \mathrm{E}+00$ & $0.00 \mathrm{E}+00$ & $0.00 \mathrm{E}+00$ & $0.00 \mathrm{E}+00$ \\
\hline MN 54 & $6.94 \mathrm{E}-02$ & $6.94 \mathrm{E}-02$ & $6.93 \mathrm{E}-02$ & $6.83 \mathrm{E}-02$ & $6.50 \mathrm{E}-02$ & $6.08 \mathrm{E}-02$ & $5.69 \mathrm{E}-02$ & $5.32 \mathrm{E}-02$ & $4.45 \mathrm{E}-02$ & $3.12 \mathrm{E}-02$ \\
\hline MN 56 & $2.37 \mathrm{E}-01$ & $2.08 \mathrm{E}-01$ & $3.74 \mathrm{E}-04$ & $5.79 \mathrm{E}-21$ & $0.00 \mathrm{E}+00$ & $0.00 \mathrm{E}+00$ & $0.00 \mathrm{E}+00$ & $0.00 \mathrm{E}+00$ & $0.00 \mathrm{E}+00$ & $0.00 \mathrm{E}+00$ \\
\hline MN 57 & 4.53E-04 & $1.11 \mathrm{E}-09$ & $0.00 \mathrm{E}+00$ & $0.00 \mathrm{E}+00$ & $0.00 \mathrm{E}+00$ & $0.00 \mathrm{E}+00$ & $0.00 \mathrm{E}+00$ & $0.00 \mathrm{E}+00$ & $0.00 \mathrm{E}+00$ & $0.00 \mathrm{E}+00$ \\
\hline MN 58 & $1.39 \mathrm{E}-06$ & $7.01 \mathrm{E}-15$ & $0.00 \mathrm{E}+00$ & $0.00 \mathrm{E}+00$ & $0.00 \mathrm{E}+00$ & $0.00 \mathrm{E}+00$ & $0.00 \mathrm{E}+00$ & $0.00 \mathrm{E}+00$ & $0.00 \mathrm{E}+00$ & $0.00 \mathrm{E}+00$ \\
\hline FE 55 & $2.80 \mathrm{E}-01$ & $2.80 \mathrm{E}-01$ & 2.79E-01 & $2.78 \mathrm{E}-01$ & $2.73 \mathrm{E}-01$ & $2.68 \mathrm{E}-01$ & $2.62 \mathrm{E}-01$ & $2.56 \mathrm{E}-01$ & $2.42 \mathrm{E}-01$ & $2.15 \mathrm{E}-01$ \\
\hline FE 59 & $5.34 \mathrm{E}-02$ & $5.34 \mathrm{E}-02$ & $5.26 \mathrm{E}-02$ & $4.79 \mathrm{E}-02$ & $3.36 \mathrm{E}-02$ & $2.12 \mathrm{E}-02$ & $1.33 \mathrm{E}-02$ & $8.41 \mathrm{E}-03$ & $2.45 \mathrm{E}-03$ & $2.09 \mathrm{E}-04$ \\
\hline $\mathrm{CO} 60$ & $5.85 \mathrm{E}-05$ & 5.85E-05 & 5.85E-05 & $5.84 \mathrm{E}-05$ & 5.79E-05 & $5.73 \mathrm{E}-05$ & $5.66 \mathrm{E}-05$ & $5.60 \mathrm{E}-05$ & 5.44E-05 & $5.14 \mathrm{E}-05$ \\
\hline CO 60M & $1.01 \mathrm{E}-03$ & $1.39 \mathrm{E}-04$ & $0.00 \mathrm{E}+00$ & $0.00 \mathrm{E}+00$ & $0.00 \mathrm{E}+00$ & $0.00 \mathrm{E}+00$ & $0.00 \mathrm{E}+00$ & $0.00 \mathrm{E}+00$ & $0.00 \mathrm{E}+00$ & $0.00 \mathrm{E}+00$ \\
\hline $\mathrm{CO} 61$ & 1.99E-06 & $1.61 \mathrm{E}-06$ & $8.32 \mathrm{E}-11$ & $0.00 \mathrm{E}+00$ & $0.00 \mathrm{E}+00$ & $0.00 \mathrm{E}+00$ & $0.00 \mathrm{E}+00$ & $0.00 \mathrm{E}+00$ & $0.00 \mathrm{E}+00$ & $0.00 \mathrm{E}+00$ \\
\hline NI 63 & $3.18 \mathrm{E}-15$ & $3.18 \mathrm{E}-15$ & $3.18 \mathrm{E}-15$ & $3.18 \mathrm{E}-15$ & $3.18 \mathrm{E}-15$ & $3.18 \mathrm{E}-15$ & $3.17 \mathrm{E}-15$ & 3.17E-15 & 3.17E-15 & $3.16 \mathrm{E}-15$ \\
\hline SUMTOT & $6.48 \mathrm{E}-01$ & $6.17 \mathrm{E}-01$ & $4.08 \mathrm{E}-01$ & $4.00 \mathrm{E}-01$ & $3.75 \mathrm{E}-01$ & $3.51 \mathrm{E}-01$ & $3.33 \mathrm{E}-01$ & $3.18 \mathrm{E}-01$ & $2.89 \mathrm{E}-01$ & $2.46 \mathrm{E}-01$ \\
\hline OTOTAL & $6.48 \mathrm{E}-01$ & $6.17 \mathrm{E}-01$ & $4.08 \mathrm{E}-01$ & $4.00 \mathrm{E}-01$ & $3.75 \mathrm{E}-01$ & $3.51 \mathrm{E}-01$ & $3.33 \mathrm{E}-01$ & $3.18 \mathrm{E}-01$ & $2.89 \mathrm{E}-01$ & $2.46 \mathrm{E}-01$ \\
\hline
\end{tabular}

